\title{
GILSON FINOTTI
}

\section{CÁLCULO EXPLÍCITO DOS TORQUES DOS ATUADORES DE UM ROBÔ PARALELO PLANO EMPREGANDO O MÉTODO DE KANE}

Dissertação apresentada à Escola

Politécnica da Universidade de

São Paulo para a obtenção do

Título de Mestre em Engenharia.

São Paulo

2008 


\section{GILSON FINOTTI}

\section{CÁLCULO EXPLÍCITO DOS TORQUES DOS ATUADORES DE UM ROBÔ PARALELO PLANO EMPREGANDO O MÉTODO DE KANE}

Dissertação apresentada à Escola Politécnica da Universidade de

São Paulo para a obtenção do

Título de Mestre em Engenharia.

Área de Concentração:

Engenharia Mecânica

Orientador:

Prof. Dr. Tarcisio Antonio Hess

Coelho

São Paulo 
Aos meus pais Mário e Tereza.

À minha esposa Eliane.

Aos meus filhos Marcelo e Luís Renato. 


\section{AGRADECIMENTOS}

Ao Prof. Dr. Tarcisio Antonio Hess Coelho por sua valiosa orientação, nobreza e dedicação.

Ao Prof. Dr. Luís Renato Abib Finotti, professor assistente de Matemática da Universidade do Tennessee, por todo apoio e ensinamentos matemáticos.

E, sobretudo, agradeço a Deus. 


\section{RESUMO}

Há mais de uma década os robôs paralelos têm atraído a atenção das comunidades acadêmica e industrial devido às suas vantagens potenciais sobre as arquiteturas predominantes - as seriais. Dentre estas vantagens, pode-se citar a leveza, as elevadas velocidades e acelerações e a capacidade de carga. A aplicação industrial mais promissora para estas arquiteturas alternativas de robôs são as operações "pega-e-põe", necessárias nas indústrias alimentícia, farmacêutica e de componentes eletrônicos. Neste trabalho apresenta-se um robô paralelo, concebido com a finalidade de realizar operações "pega-e-põe" no espaço bidimensional (plano). O objetivo principal é a análise dinâmica deste mecanismo, empregando o método de Kane, para a determinação dos torques dos atuadores e das forças de reação, causados pelo efeito dinâmico de sua movimentação, quando a garra esteja sujeita a esforços externos e realizando uma trajetória retilínea ou circular em movimento uniforme ou uniformemente variado. Para tanto, desenvolveu-se nesta dissertação a análise cinemática do robô, um estudo de possíveis trajetórias para a garra, o levantamento do espaço de trabalho, bem como a análise dinâmica correspondente. Incluiu-se também diversas simulações para caracterizar melhor suas propriedades.

Palavras-chave: Método de Kane. Robô paralelo. Cinemática. Estática. Dinâmica. 


\begin{abstract}
For over a decade parallel robots have attracted the interest from academic and industrial communities due to their potential advantages over the predominant serial architecture. Among these advantages are the lighter weight and higher speeds, accelerations, and load capacity. The most promising industrial application for these alternative architectures are the pick-and-place operations, which are needed in food, pharmaceutical and electronics industries. We show here a parallel robot designed to perform pick-and-place operations in two dimensions , i.e., on a plane. The main goal is the dynamical analysis of this mechanism by means of the Kane method. We determine the torques of the actuators and the reaction forces caused by the dynamical effects of its movement, when its end-effector is subject to external load. The cases of uniform and accelerated movements, with either straight or circular trajectory, are considered. Therefore, in this dissertation we present the kinematics analysis of the robot, an analysis of possible end-effector trajectories, the workspace development, and the corresponding dynamical analysis. A few simulations are also included to better describe its properties.
\end{abstract}

Keywords: Kane method. Parallel robot. Statics. Kinematics. Dynamics. 


\section{LISTA DE FIGURAS}

1.1 Diagrama cinemático do mecanismo 2 RRR+RP . . . . . . . 20

2.1 Mecanismo paralelo . . . . . . . . . . . . . . . . . 23

2.2 Robô Delta da ABB . . . . . . . . . . . . . . . . . . 23

2.3 Tipos de juntas . . . . . . . . . . . . . . . . . 24

2.4 Diagrama cinemático do manipulador robótico paralelo espacial 3GM . . . . . . . . . . . . . . . . . 31

$2.5 \quad$ Fluxograma . . . . . . . . . . . . . . . 34

3.1 Mecanismo: coordenada dos vértices. . . . . . . . . . . . 35

4.1 Diagrama do mecanismo . . . . . . . . . . . . . . . . . . 42

4.2 Espaço de trabalho método discretização . . . . . . . . . . . . . 44

4.3 Curva externa do espaço de trabalho . . . . . . . . . . . . . 46

4.4 Curva interna e área de trabalho . . . . . . . . . . . . . . . 46

4.5 Otimização do espaço de trabalho . . . . . . . . . . . . . . . . . 48

5.1 Trajetória retilínea: curva tempo x velocidade. . . . . . . . . 51

5.2 Trajetória retilínea: curva espaço x velocidade. . . . . . . . . 53

5.3 Trajetória circular com centro do arco na origem dos eixos de coordenadas. . . . . . . . . . . . . . . 57

5.4 Trajetória circular caso geral . . . . . . . . . . . . . . . . . . . . . . . . . . 64

5.5 Trajetória circular, detalhe . . . . . . . . . . . 65

6.1 Diagrama cinemático do mecanismo . . . . . . . . . . 68

6.2 Dimensões e ângulos dos elos . . . . . . . . . . . . . . . . 69

6.3 Diagrama auxiliar para determinação das acelerações . . . . . . 72

7.1 Forças e momentos . . . . . . . . . . . . . . 76

$7.2 \quad$ Diagrama de corpo livre: elo $7 \ldots \ldots$. . . . . . . . 77

7.3 Diagrama de corpo livre: elo $4 \ldots \ldots$. . . . . . . . 78

$7.4 \quad$ Diagrama de corpo livre: elo $3 \ldots \ldots$. . . . . . . . 79

7.5 Diagrama de corpo livre: elo $2 \ldots \ldots$. . . . . . . . . 80

7.6 Diagrama de corpo livre: elo $5 \ldots \ldots$. . . . . . . . 81

7.7 Diagrama de corpo livre: elo $6 \ldots \ldots$. . . . . . . . . 82

7.8 Determinação dos contrapesos . . . . . . . . . . . . . . 83

8.1 Diagrama cinemático do mecanismo . . . . . . . . . . 88

8.2 Diagramas de corpo livre dos elos A e B . . . . . . . . . . 89

8.3 Diagramas de corpo livre dos elos D e E . . . . . . . . . 96

8.4 Diagramas de corpo livre dos elos H e J . . . . . . . . . . . 101

8.5 Mecanismo: reações finais nos atuadores . . . . . . . . . . . 111 
8.6 Exemplo 3: trajetória da garra . . . . . . . . . . . . . 113

8.7 Exemplo 3: curvas dos torques nos atuadores . . . . . . . . . 114

9.1 Diagramas dos elos H e J . . . . . . . . . . . . . . 117

9.2 Exemplo 1: curvas dos torques com e sem atrito . . . . . . . 120

9.3 Exemplo 2: curvas dos torques com e sem atrito . . . . . . . . 121

10.1 Posições das juntas A e D . . . . . . . . . . . . . . . . . . . 122

10.2 Propriedades do produto vetorial . . . . . . . . . . . . . 123

10.3 Interseção das retas PQ e RS . . . . . . . . . . . . . . . . . . . 124

10.4 Balanceamento com contrapesos . . . . . . . . . . . . 127

11.1 Anexo A: diagrama cinemático . . . . . . . . . . . . . . . 136

11.2 Anexo B: diagrama cinemático . . . . . . . . . . . . . . 140

11.3 Anexo E: trajetória circular com centro na origem . . . . . . . . 148

11.4 Anexo F: trajetória circular geral . . . . . . . . . . . . . . . . 152

11.5 Anexo G: diagrama cinemático . . . . . . . . . . . . . . . . 159

11.6 Anexo $\mathrm{H}$ : diagrama cinemático . . . . . . . . . . . . . . . . . . . . . . . . . . . . . . . . 165

11.7 Anexo I: diagrama cinemático . . . . . . . . . . . . . . . 165

11.8 Anexo J: diagrama cinemático . . . . . . . . . . . . . . . . 168

11.9 Anexo J: diagramas de corpo livre . . . . . . . . . . . . . 168

11.10 Anexo K: diagrama de corpo livre do elo A . . . . . . . . . . . 171

11.11 Anexo L: diagrama de corpo livre do elo D . . . . . . . . . . . . 173

11.12 Anexo M: diagrama de corpo livre da cadeia passiva . . . . . . 175

11.13 Anexo N: diagrama cinemático . . . . . . . . . . . . 176

11.14 Anexo O: diagramas de corpo livre . . . . . . . . . . . . . . 179

11.15 Anexo P: diagrama de corpo livre da cadeia passiva . . . . . . . 183

11.16 Anexo Q: diagrama cinemático . . . . . . . . . . . . 185

11.17 Anexo S: diagrama cinemático . . . . . . . . . . . . . . 190 


\section{LISTA DE TABELAS}

$3.1 \quad$ Exemplo cinemática inversa. . . . . . . . . . . . . . . 37

8.1 Exemplo de aplicação 1. Análise dinâmica . . . . . . . . . . . . 111

8.2 Exemplo de aplicação 2. Análise dinâmica . . . . . . . . . . . . 112

8.3 Exemplo de aplicação 3. Análise dinâmica . . . . . . . . . . . . 113

9.1 Exemplo de aplicação 1. Atrito . . . . . . . . . . . . . . . . 119

9.2 Exemplo de aplicação 2. Atrito . . . . . . . . . . . . . . 120

10.1 Comparação entre formulações . . . . . . . . . . . . . . . . . . . . . . . . . . . 126

10.2 Influência dos contrapesos conforme a velocidade . . . . . . . 128

10.3 Torques com e sem acelerações e $\mathrm{v}=1 \mathrm{~m} / \mathrm{s} \ldots \ldots$. . . . . . . 128

10.4 Torques com e sem acelerações e $\mathrm{v}=4 \mathrm{~m} / \mathrm{s}$. . . . . . . . . . . 129

10.5 Acelerações tangenciais e de Coriolis . . . . . . . . . . . . . . 130

10.6 Influência da aceleração de Coriolis em T1D . . . . . . . . . . . 130

10.7 Influência da aceleração de Coriolis em T1 . . . . . . . . . . . 131

10.8 Exemplo de aplicação 1 sem e com atrito . . . . . . . . . . . . 132

10.9 Torque e atrito na guia linear . . . . . . . . . . . . . . . 132

10.10 Influência do atrito em T1D . . . . . . . . . . . . . . . . 133

10.11 Influência do atrito em $\mathrm{T} 1$. . . . . . . . . . . . . . 133

10.12 Influência do atrito com carga externa em T1 . . . . . . . . . . 133

10.13 Influência do atrito com carga externa em T1D . . . . . . . . 133 


\title{
LISTA DE ABREVIATURAS E SIGLAS
}

\author{
CG - Centro de gravidade \\ GL - Graus de liberdade \\ GM - Graus de mobilidade \\ TMA - Teorema do momento angular \\ TMB - Teorema do movimento do baricentro
}




\section{LISTA DE SÍMBOLOS}

$\overrightarrow{A_{i}} \quad$ aceleração linear do centro de massa do corpo $i$

$\overrightarrow{A_{J}} \quad$ aceleração linear do CG do elo $J$ (se o índice for $A$ refere-se ao elo $A$, etc. Isto é válido para os demais símbolos que se seguem)

$A_{J}^{n} \quad$ módulo da componente de $\overrightarrow{A_{J}}$ na direção normal à curva

$A_{J}^{t} \quad$ módulo da componente de $\overrightarrow{A_{J}}$ na direção tangente à curva

$\vec{a} \quad$ aceleração linear

$\overrightarrow{a_{x}}, \overrightarrow{a_{y}} \quad$ componentes da aceleração do mecanismo

$\overrightarrow{\alpha_{i}} \quad$ aceleração angular do centro de massa do corpo $i$

$\beta \quad$ ângulo de inclinação da força $\vec{F}$

C junta cilíndrica, módulo da força de atrito de Coulomb

$C_{k} \quad$ conectividade da cadeia ativa $k$

$C_{n+1} \quad$ conectividade da cadeia passiva

c coeficiente de atrito viscoso

$c \theta_{n}, c_{n} \quad$ coseno do ângulo $\theta_{n}$

d diâmetro da junta de rotação

$d_{p} \quad$ comprimento da junta prismática

$\vec{F} \quad$ força externa aplicada à garra

$F_{a} \quad$ módulo da resultante da força de atrito

$\vec{F}_{1}, \vec{F}_{2} \quad$ componentes da força de reação no atuador $O_{6}$

$\overrightarrow{F_{1 D}}, \overrightarrow{F_{2 D}} \quad$ componentes da força de reação no atuador $O_{2}$

$\overrightarrow{F_{1 H}}, \overrightarrow{F_{2 H}} \quad$ componentes da força de reação na junta $O_{7}$

$F_{p r} \quad$ módulo da força de atrito da proteção da junta prismática

$\phi \quad$ ângulo de inclinação da trajetória

$I_{i} \quad$ momento de inércia do corpo $i$ em relação ao seu eixo baricêntrico 
$J_{x} \quad$ matriz jacobiana da cinemática direta

$J q \quad$ matriz jacobiana da cinemática inversa

$k \quad$ coeficiente de atrito de Coulomb

$k_{p} \quad$ coeficiente de atrito da junta prismática

$k_{r} \quad$ coeficiente de atrito de rolamento

L lagrangeana

$L_{n} \quad$ comprimento do elo $n$

$L_{n G} \quad$ posição do CG do elo $n$

$L_{n P} \quad$ posição do CG do contrapeso elo $n$

$\lambda \quad$ número de movimentos independentes que uma peça pode executar num determinado espaço sem vínculos

M mobilidade do mecanismo, ou quantidade de variáveis de movimento de Kane

$\vec{M} \quad$ momento externo aplicada à garra

$m_{i} \quad$ massa do corpo $i$

n número de peças de um mecanismo ou quantidade de corpos de um sistema

$n_{j i} \quad$ número de juntas com grau de liberdade $i$

$\overrightarrow{\omega_{i}} \quad$ velocidade angular do centro de massa do corpo $i$

$P \quad$ junta prismática

$Q_{k} \quad$ força generalizada

$q_{k} \quad$ coordenada generalizada

$R \quad$ junta de rotação

$\vec{R}_{i} \quad$ resultante das forças atuantes no corpo $i$

$S \quad$ junta esférica

$s_{A B} \quad$ espaço percorrido entre os pontos $A$ e $B$ da trajetória (se o índice for $B C$, refere-se ao espaço entre os potos $B$ e $C$, etc) 


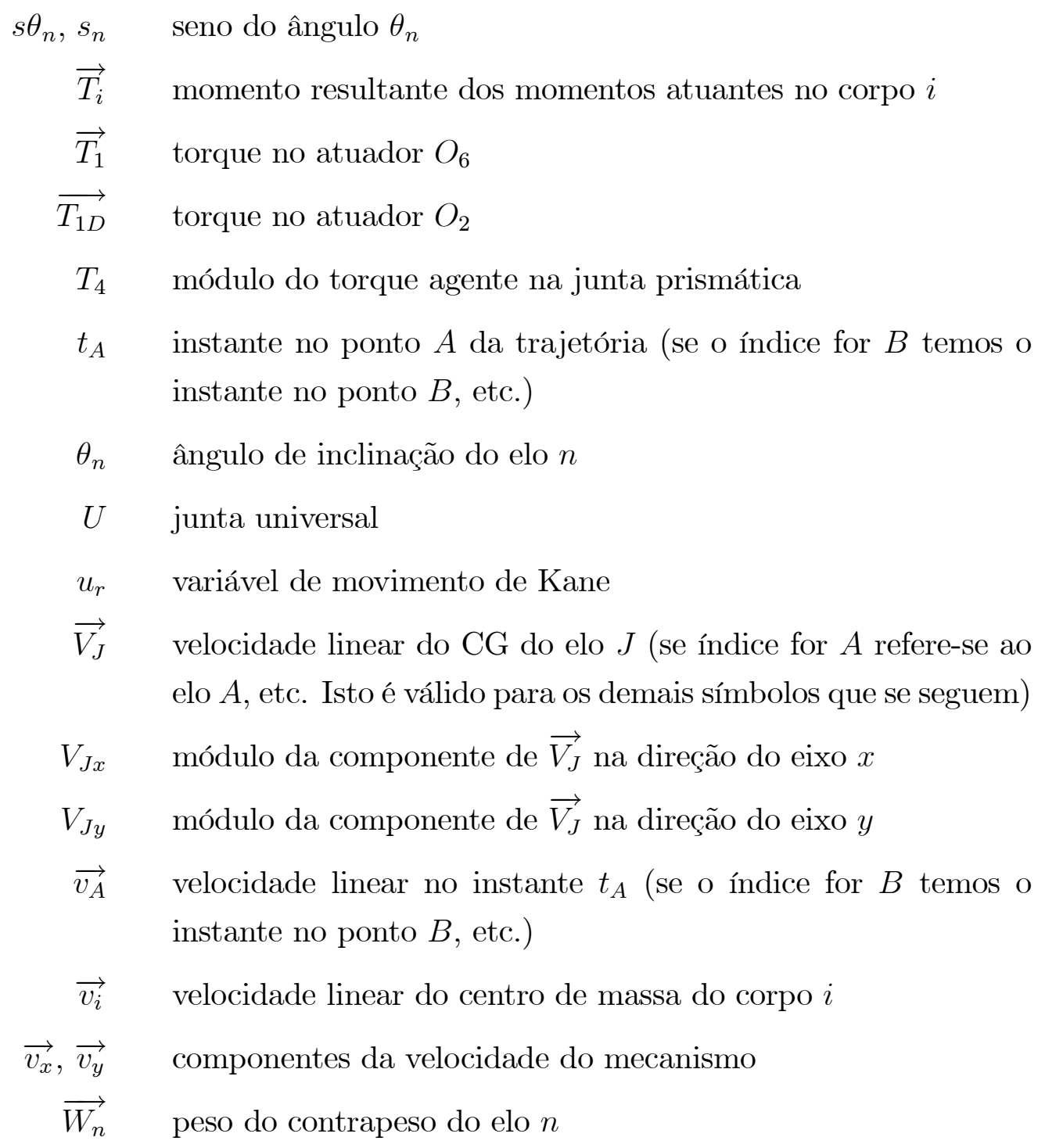




\section{SUMÁRIO}

1 Introdução $\quad 19$

1.1 Considerações preliminares . . . . . . . . . . . . . . . . . . 19

1.2 Objetivos . . . . . . . . . . . . . . . . . 19

1.3 Descrição do mecanismo . . . . . . . . . . . . . . . . . 20

1.4 Sobre a divisão do trabalho . . . . . . . . . . . . . . . . . . 21

2 Revisão bibliográfica e metodologia empregada 22

2.1 Mecanismos . . . . . . . . . . . . . . . . . . . . 22

2.2 Mecanismo serial e paralelo . . . . . . . . . . . . . . 22

2.3 Componentes dos mecanismos . . . . . . . . . . . . . . . 24

2.4 Nomenclatura . . . . . . . . . . . . . . . . . . . . . 25

2.5 Mobilidade do mecanismo . . . . . . . . . . . . . . 25

2.6 Análise cinemática . . . . . . . . . . . . . . . . 26

2.7 Espaço de trabalho . . . . . . . . . . . . . . . . . 26

2.8 Análise dinâmica . . . . . . . . . . . . . . . . . . . . . 27

2.9 Métodos para análise dinâmica . . . . . . . . . . . . . . . 28

2.9 .1 Método de Newton-Euler . . . . . . . . . . . . . . . . 28

2.9.2 Método de Lagrange . . . . . . . . . . . . . . . . . . . . 29

2.9 .3 Método de Kane . . . . . . . . . . . . . . . . . . . . . 29

2.10 Síntese. Método da adição de uma cadeia passiva. . . . . . . . . . 30

2.11 Metodologia empregada . . . . . . . . . . . . . . . 32

3 Análise cinemática: posições 35

3.1 Cinemática inversa . . . . . . . . . . . . . . . . . . 35

3.2 Cinemática direta . . . . . . . . . . . . . . . . . . 37

3.3 Singularidades . . . . . . . . . . . . . . . . 38

3.3 .1 Introdução . . . . . . . . . . . . . . . . . . 38

3.3.2 Determinação das matrizes jacobianas . . . . . . . . . 39

4 Estudo do espaço de trabalho $\quad 42$

4.1 Método por discretização . . . . . . . . . . . . . . . . . . . . . . 42

4.2 Método geométrico . . . . . . . . . . . . . . . . . 44

4.2.1 Curva externa da área de trabalho. . . . . . . . . . . . . 44

4.2.2 Curva interna da área de trabalho . . . . . . . . . . . . 46

4.3 Otimização do espaço de trabalho . . . . . . . . . . . . . . . . . 47 
5 Trajetórias da garra $\quad \mathbf{5 0}$

5.1 Introdução . . . . . . . . . . . . . . . . . . . . . . 50

5.2 Deslocamentos retilíneos . . . . . . . . . . . . . . 50

5.2 .1 Primeira condição: $s_{B C} \geqslant 0 \ldots \ldots \ldots$. . . . . . 53

5.2 .2 Segunda condição: $s_{B C}<0$. . . . . . . . . . 54

5.3 Deslocamento retilíneo numa direção qualquer . . . . . . . . . . . 54

5.3.1 Primeira condição: $s_{B C} \geqslant 0 \ldots \ldots \ldots$......... 54

5.3 .2 Segunda condição: $s_{B C}<0 \ldots \ldots \ldots \ldots 5$

5.4 Deslocamento circular . . . . . . . . . . . . . . . . . . 57

5.5 Centro do arco na origem dos eixos de coordenadas . . . . . . . . 58

5.5.1 Primeira condição: $s_{B C} \geqslant 0 \ldots \ldots \ldots$. . . . . 58

5.5.2 Segunda condição: $s_{B C}<0 \ldots \ldots \ldots$........... 61

5.6 Centro do arco fora da origem dos eixos de coordenadas. Caso geral. 63

6 Análise cinemática: velocidades e acelerações $\quad 68$

6.1 Introdução . . . . . . . . . . . . . . . . . . . . . . . . 68

6.2 Velocidades e acelerações angulares . . . . . . . . . . . . . 68

6.2.1 Cadeia passiva . . . . . . . . . . . . . . . 69

6.2 .2 Cadeia ativa direita . . . . . . . . . . . . 70

6.2 .3 Cadeia ativa esquerda . . . . . . . . . . . . . 71

6.3 Acelerações dos CG dos elos . . . . . . . . . . . . . . . . . 72

6.3.1 Elo A: $A_{A}^{n}$ e $A_{A}^{t} \ldots \ldots \ldots . \ldots . \ldots 72$

6.3 .2 Elo B: $A_{B}^{n}$ e $A_{B}^{t} \ldots \ldots \ldots \ldots 73$

6.3.3 Elo D: $A_{D}^{n}$ e $A_{D}^{t} \ldots \ldots \ldots \ldots 74$

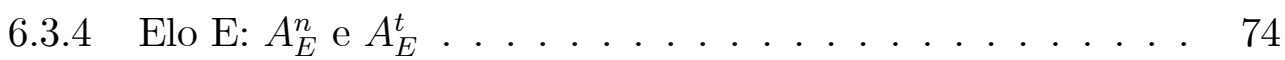

6.3.5 Elo H: $A_{H}^{n}$ e $A_{H}^{t} \ldots \ldots \ldots \ldots . \ldots . \ldots . \ldots 74$

6.3 .6 Elo J: $A_{J}^{n}$ e $A_{J}^{t} \ldots \ldots \ldots \ldots 74$

$\begin{array}{llr}7 & \text { Análise estática } & \mathbf{7 6}\end{array}$

7.1 Introdução . . . . . . . . . . . . . . . . . . . . . . . . . . . . . . 76

7.2 Equações de equilíbrio . . . . . . . . . . . . . . . . . . 77

7.2.1 Listagem do programa . . . . . . . . . . . . . 82

7.3 Determinação dos contrapesos para momentos de reação nulos . 82

8 Análise dinâmica $\quad 85$

8.1 Introdução . . . . . . . . . . . . . . . . . . . . 85

8.2 Aplicação do método de Kane . . . . . . . . . . . . . . . . . 87

8.2 .1 Especificação das variáveis. . . . . . . . . . . . . . 88 
8.2 .2 Elos A e B . . . . . . . . . . . . . . . 88

8.2 .3 Elo A . . . . . . . . . . . . . . . . . . 89

8.2 .4 Elo B . . . . . . . . . . . . . . . . . . . . . . . 92

8.2.5 Resultado para os elos A e B . . . . . . . . . . . 95

8.2 .6 Elos D e E . . . . . . . . . . . . . . . 95

8.2 .7 Elo D . . . . . . . . . . . . . . 96

8.2 .8 Elo E . . . . . . . . . . . . . . . . . . . . . . . . . . . . . 98

8.2.9 Resultado para os elos D e E . . . . . . . . . . . . 100

8.2.10 Elos H e J . . . . . . . . . . . . . . . . . . . . 100

8.2 .11 Elo $\mathrm{H} \ldots \ldots \ldots$. . . . . . . . . . . . . . . . . . . . . . 101

8.2 .12 Elo J . . . . . . . . . . . . . . . . . . . . . . . 103

8.2.13 Resultado para os elos H e J . . . . . . . . . . . . . 105

8.2.14 Resultado geral . . . . . . . . . . . . . . . 106

8.3 Determinação dos torques e forças . . . . . . . . . . . . . 106

8.3.1 Torques de reação $T_{1}$ e $T_{1}^{D} \ldots \ldots \ldots$. . . . . . . 106

8.3.2 Forças de reação $F_{1}$ e $F_{2} \ldots \ldots$. . . . . . . 107

8.3.3 Forças de reação $F_{1}^{D}$ e $F_{2}^{D} \ldots \ldots$. . . . . . . . 107

8.3.4 Forças e torque de reação na cadeia passiva . . . . . . . . 108

8.4 Roteiro de cálculo . . . . . . . . . . . . . . . . . . . . . . 108

8.4.1 Aglutinação dos programas. Anexo Q . . . . . . . . . . . . 110

8.4.2 Exemplo de aplicação 1 . . . . . . . . . . . . . . . . . . . 111

8.4.3 Exemplo de aplicação 2 . . . . . . . . . . . . . . . . . . . 112

8.4.4 Exemplo de aplicação 3 . . . . . . . . . . . . . . . . . . . 112

9 Efeito do atrito nas juntas $\quad 115$

9.1 Introdução . . . . . . . . . . . . . . . . . . . . 115

9.2 Desenvolvimento das equações . . . . . . . . . . . . . . . . 116

9.2 .1 Elo H . . . . . . . . . . . . . . . . . . . . . . 116

9.2 .2 Elo J . . . . . . . . . . . . . . . . . . . . . . . 117

9.2.3 Resultado para os elos H e J . . . . . . . . . . . . . . 118

9.2.4 Força de atrito na junta prismática . . . . . . . . . . . . . 118

9.2.5 Resultado geral . . . . . . . . . . . . . . . . . . . 119

9.2.6 Exemplo de aplicação 1 . . . . . . . . . . . . . . . . . . 119

9.2.7 Exemplo de aplicação 2 . . . . . . . . . . . . . . . . . . 120

10 Discussão 122

10.1 Análise cinemática: posições . . . . . . . . . . . . . . . . . 122

10.1 .1 Cinemática inversa . . . . . . . . . . . . . . 122 
10.1.2 Cinemática direta . . . . . . . . . . . . . . . . . 124

10.2 Análise dinâmica . . . . . . . . . . . . . . . . . . . . . . 125

10.2.1 Comparação entre o método de Kane com os de Newton-

Euler e Lagrange . . . . . . . . . . . . . . . . . 125

10.2.2 O balanceamento com contrapesos . . . . . . . . . . 126

10.2.3 Influência das acelerações dos CG dos elos. . . . . . . . . . 128

10.2.4 A influência da aceleração de Coriolis . . . . . . . . . . . . 129

10.3 Efeito do atrito nas juntas . . . . . . . . . . . . . . . 131

11 Conclusões 135

$\begin{array}{ll}\text { Anexo A: Programa cinemática inversa } & 136\end{array}$

$\begin{array}{ll}\text { Anexo B: Programa cinemática direta } & 140\end{array}$

Anexo C: Programa espaço de trabalho 144

Anexo D: Progr. vel. e acel., traj. ret. 146

Anexo E: Progr. vel. e acel., traj. circ. e centro na origem 148

Anexo F: Prog. vel. e acel., traj. circ. geral 152

Anexo G: Programa equações das velocidades e acelerações $\quad 158$

Anexo H: Programa cálculo velocidades e acelerações 161

Anexo I: Programa análise estática 165

Anexo J: Programa equações dinâmica $1 \quad 168$

$\begin{array}{ll}\text { Anexo K: Programa equações dinâmica 2 } & 171\end{array}$

Anexo L: Programa equações dinâmica $3 \quad 173$

Anexo M: Programa equações dinâmica 4

Anexo N: Programa dos torques dinâmicos de reação 176

Anexo O: Programa das forças dinâmicas nas cadeias ativas $\quad 179$

Anexo P: Progr. forças e torque dinâmicos na cadeia passiva 183

Anexo Q: Programa total dos torques e forças de reação 185 
Anexo R: Programa equações dinâmica 5

Anexo S: Programa dos torques de reação com atrito

Referências Bibliográficas 


\section{Capítulo 1: INTRODUÇÃO}

\subsection{Considerações preliminares}

Embora a arquitetura predominante em robôs manipuladores ainda seja a do tipo serial, tem-se observado nas últimas décadas uma diminuição pequena mas gradativa nesta preferência em favor da arquitetura paralela. Pelo fato desta ser constituída de várias cadeias (pernas) atuando simultaneamente sobre a plataforma móvel (órgão terminal) em vez de uma só, como na arquitetura serial, ela possui algumas vantagens potenciais como leveza, velocidades e acelerações elevadas, e maior capacidade de carga. Suas principais desvantagens, entretanto, referem-se ao espaço de trabalho inferior e à maior complexidade mecânica de seus componentes, ou seja, maior número de elos e de juntas. Os mecanismos paralelos começaram a ser desenvolvidos na década de 60 por Gough e Whitehall numa máquina de teste de pneus e em seguida por Stewart como simulador de vôos. No entanto, apenas nas duas últimas décadas outras aplicações, como robôs manipuladores e máquinas ferramentas, passaram a interessar o desenvolvimento conduzido em universidades, centros de pesquisa e indústrias.

A grande maioria dos artigos sobre os mecanismos paralelos se dedicam mais à abordagem cinemática do que dinâmica. Neste nosso trabalho, entretanto, enfatizaremos a análise dinâmica e nesta análise utilizaremos o método de Kane para o caso específico de um mecanismo paralelo plano tipo $2 \underline{R} R R+R P$, esquematicamente mostrado na fig.1.1 e descrito adiante. Este mecanismo é derivado de um manipulador robótico paralelo espacial de 3 GM para operações "pegae-põe"(Pick-And-Place), mostrado na fig.2.4 e criado pelo Prof. Dr. Tarcisio Antonio Hess Coelho, do qual tomamos sua principal característica na versão plana para focar nossa análise[1].

\subsection{Objetivos}

Um dos objetivos deste trabalho é o estudo cinemático-dinâmico do mecanismo acima referido nas situações mais diversas quando sua garra está sujeita a esforços (torque e força) e a movimentação uniforme ou variada. Faz parte deste estudo não só sua análise teórica como também a elaboração de algoritmos para a solução numérica de cada tópico abordado, principalmente para a determinação dos torques nos atuadores e das forças de reação na base do mecanismo quando o 
mesmo realiza uma tarefa, isto é, nas condições de carga e de movimentação, seja em trajetória reta ou circular, seja em movimento uniforme ou uniformemente variado, definido por rampas de aceleração e desaceleração.

É também nosso propósito mostrar uma aplicação prática do método de Kane e termos uma idéia de suas vantagens e desvantagens sobre os demais métodos.

\subsection{Descrição do mecanismo}

Conforme mostra a fig.1.1 o mecanismo $2 \underline{R} R R+R P$ é composto de duas cadeias ativas $O_{2} A B$ e $O_{6} D C$ e de uma cadeia passiva $O_{7} G$. As cadeias ativas possuem atuadores respectivamente localizados nas articulações $O_{2}$ e $O_{6}$, e todas suas juntas são de rotação. A cadeia passiva possui uma junta de rotação próxima à base e uma junta prismática no meio de seu comprimento. A parte superior da cadeia passiva é engastada na plataforma móvel em $G$ segundo um ângulo de $90^{\circ}$. Desta forma a cadeia passiva pode sofrer uma rotação em torno de $O_{7}$ e seu comprimento $L_{7}$ é variável. A finalidade deste mecanismo é ser utilizado como um manipulador robótico cujo ponto de referência da garra (efetuador) é o ponto G.

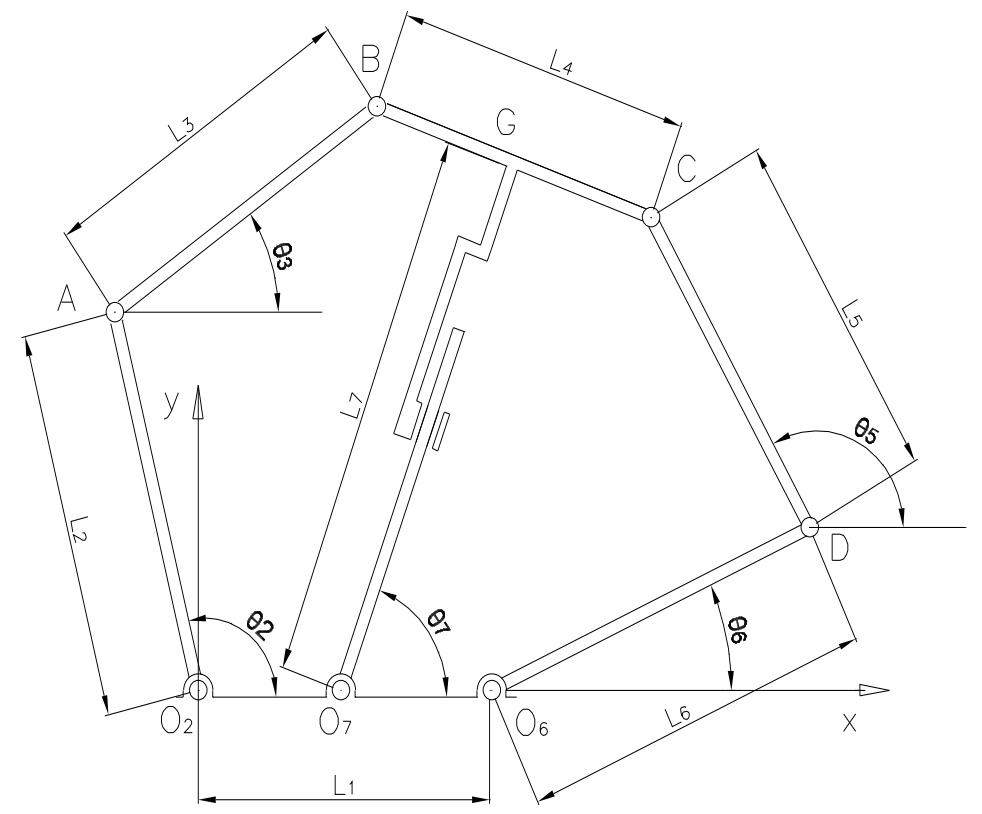

Figura 1.1: Diagrama cinemático do mecanismo $2 \mathrm{RRR}+\mathrm{RP}$ 


\subsection{Sobre a divisão do trabalho}

Já vimos neste Capítulo 1 os nossos objetivos e uma descrição do mecanismo que abordaremos.

O Capítulo 2 tratará de uma revisão bibliográfica e da metodologia que empregaremos neste trabalho.

No Capítulo 3 desenvolveremos a análise cinemática direta e inversa de posições e um estudo de singularidades.

No Capítulo 4 estudaremos o espaço de trabalho pelo método por discretização e pelo método geométrico; e em seguida desenvolveremos um estudo da otimização do espaço de trabalho.

No Capítulo 5 realizaremos um estudo sobre a trajetória retilínea e circular da garra do mecanismo, considerando as fases de aceleração, de movimento uniforme e desaceleração.

No Capítulo 6 desenvolveremos um estudo da análise cinemática para a determinação das velocidades e acelerações dos elos.

No Capítulo 7 desenvolveremos a análise estática das forças e momentos atuantes e resultantes do mecanismo inclusive considerando a existência de contrapesos para o balanceamento estático.

No Capítulo 8 desenvolveremos a análise dinâmica utilizando o método de Kane, explicando roteiro de cálculo e apresentando exemplos de aplicação.

No Capítulo 9 trataremos de um estudo dinâmico do mecanismo, considerando a existência de atrito de Coulomb na junta prismática da cadeia passiva.

E finalmente o Capítulo 10 apresentará uma discussão sobre vários tópicos de nosso trabalho e o Capítulo 11 a conclusão.

Nos Anexos de $\mathbf{A}$ até $\mathbf{S}$ apresentaremos os programas elaborados no software Mathematica para o desenvolvimento de equações e aplicações numéricas das teorias apresentadas nos capítulos acima. 


\section{Capítulo 2: REVISÃO BIBLIOGRÁFICA E METODOLOGIA EMPREGADA}

\subsection{Mecanismos}

Podemos dizer que um mecanismo é um dispositivo inventado pelo homem para substituir seu trabalho braçal principalmente nas atividades repetitivas. Ele é capaz de aumentar a força aplicada, a velocidade de operação, melhorar a precisão de posicionamento, pode trabalhar em ambientes inóspitos e inacessíveis ao homem, enfim é capaz de sobrepujar sobejamente a nossa eficiência manual. Trata-se, portanto, de um recurso cada vez mais aprimorado e aplicado nas mais diversas áreas graças não só ao seu retorno econômico como tecnológico.

Releaux define o mecanismo como uma combinação de corpos rígidos ou resistentes de tal forma conectados que se movem um em relação ao outro com movimento relativo definido[2]. Segundo Suh e Radcliffe "o mecanismo é destinado tipicamente para criar um determinado movimento de um corpo rígido em relação a um membro de referência" [3].

\subsection{Mecanismo serial e paralelo}

Basicamente os mecanismos são classificados em duas categorias: serial e paralelo. O serial possui uma única cadeia cinemática aberta. Uma de suas extremidades é fixada numa base e a outra extremidade possui uma garra ou punho para prender uma ferramenta e se movimenta para realizar o trabalho programado. Os mecanismos paralelos possuem várias cadeias cinemáticas formando o que se chama de cadeia fechada, como mostrado esquematicamente na fig.2.1. Do mesmo modo que o mecanismo serial, de um lado possui a garra ou punho os quais são fixados na plataforma móvel (ou órgão terminal) que pode se movimentar para a realização do trabalho. Esta plataforma móvel está ligada à base por pelo menos duas cadeias cinemáticas. Segundo Merlet, a estrutura cinemática paralela é definida como um mecanismo de cadeia fechada em que a plataforma móvel (ou órgão terminal) encontra-se conectada a uma base fixa por, pelo menos, duas cadeias cinemáticas independentes[4].

O fato dos atuadores poderem estar localizados na base ou próximos dela, é um fator positivo dos mecanismos paralelos pois isto lhes confere maior rigidez já que a inércia das partes diminui. A maior rapidez é também outra vantagem 


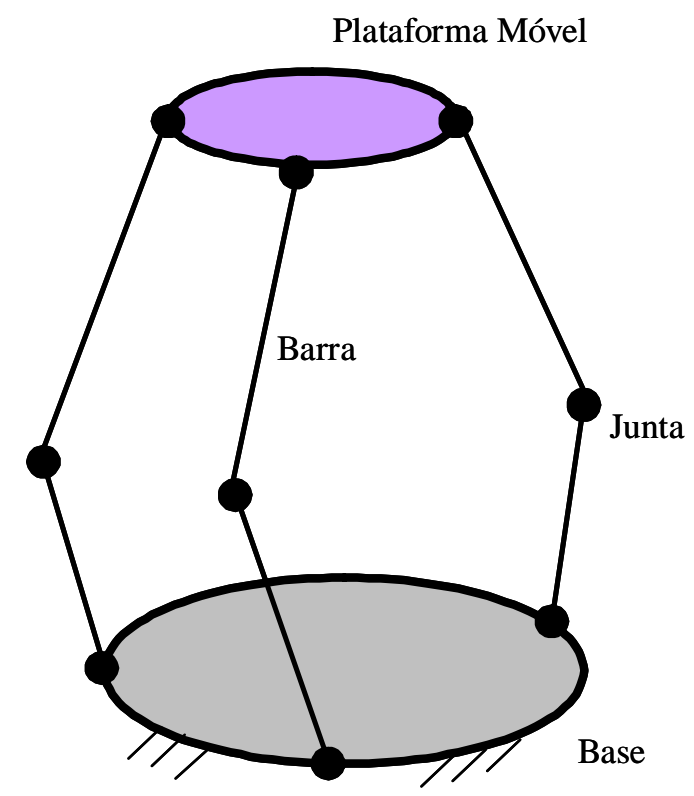

Figura 2.1: Mecanismo paralelo

dos mecanismos paralelos. Entre suas desvantagens podemos citar a limitação do espaço de trabalho, a maior complexidade da cinemática direta e a maior probabilidade de ocorrências de singularidades.

A fig. 2.2 mostra um exemplo de robô industrial para operações Pick-AndPlace, com arquitetura paralela, desenvolvido pela Asea Brown Boveri denominado FlexPicker ou robô Delta.

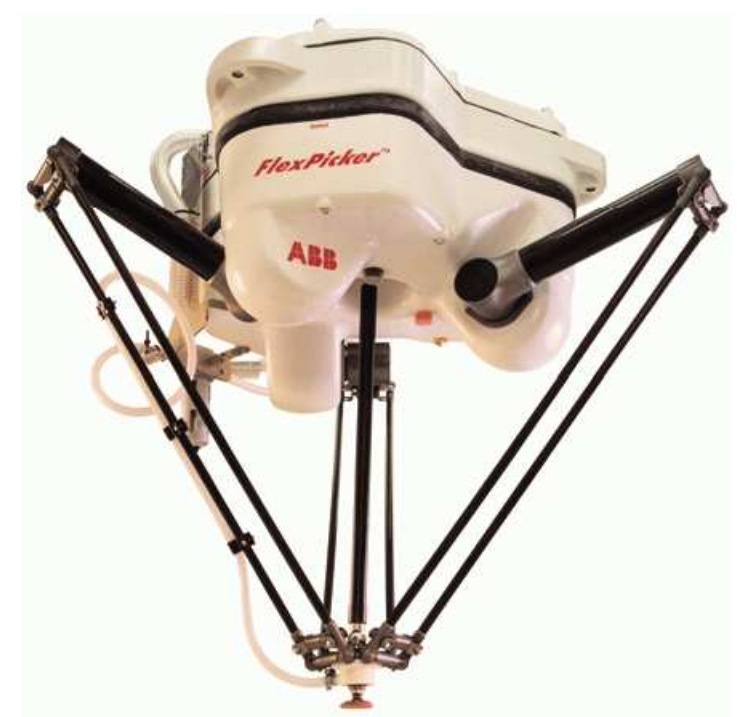

Figura 2.2: Robô Delta da ABB 


\subsection{Componentes dos mecanismos}

A base do mecanismo é sua parte fixa sobre a qual são montadas as cadeias cinemáticas. A cadeia cinemática é um conjunto de peças ou elos conectados por juntas ou pares cinemáticos. A cadeia cinemática é chamada de ativa quando possui um ou mais atuadores, senão, ela é chamada de passiva. As juntas ou pares cinemáticos são caracterizados pelos seus graus de liberdade. A fig.2.3 de Suh e Radcliffe mostra os tipos de juntas mais utilizados com seus respectivos graus de liberdade e representação esquemática [3]. As juntas de rotação (representadas por R) e as juntas prismáticas (representadas por P) possuem somente um grau de liberdade. As juntas cilíndricas (representadas por C) e as juntas universais (representadas por U) possuem dois graus de liberdade e as juntas esféricas (representadas por S) possuem três graus de liberdade. Os atuadores são dispositivos que transmitem movimento às cadeias ativas. A plataforma móvel (ou órgão terminal) é a parte do mecanismo que está ligada à base pelas cadeias cinemáticas e é ela que realiza o movimento de saída programado.

\begin{tabular}{|c|c|c|c|}
\hline Nome do par cinemático & Forma geométrica & $\begin{array}{l}\text { Representações } \\
\text { esquemáticas }\end{array}$ & $\begin{array}{r}\text { Graus de } \\
\text { liberdade }\end{array}$ \\
\hline 1. Rotação (R) & & & 1 \\
\hline 2. Cilíndrico (C) & & & 2 \\
\hline 3. Prismático $(\mathbf{P})$ & & & 1 \\
\hline 4. Esférico (S) & & & 3 \\
\hline 5. Universal (U) & & & 2 \\
\hline 6. Helicoidal (H) & & & 1 \\
\hline
\end{tabular}

Figura 2.3: Tipos de juntas 


\subsection{Nomenclatura}

A notação literal que identifica um mecanismo é feita utilizando-se as letras que representam as juntas, na seqüência em que estão montadas em cada cadeia cinemática, começando pela junta mais próxima da base[5]. Se a junta é ativa, ou seja, se está acoplada a um atuador, então a letra que a representa deve ser sublinhada. O conjunto de letras que representa uma determinada cadeia cinemática deve ser precedido pelo número referente à quantidade da respectiva cadeia cinemática existente no mecanismo. No caso, por exemplo, do mecanismo objeto de nosso estudo, temos duas cadeias ativas constituídas de três juntas de rotação sendo que a primeira é ativa e temos ainda uma cadeia passiva constituída de uma junta de rotação e uma junta prismática. Sua nomenclatura é então: $2 \underline{R} R R+R P$.

\subsection{Mobilidade do mecanismo}

A mobilidade de um mecanismo refere-se ao número de movimentos independentes possíveis que o órgão terminal do mecanismo pode executar, no seu espaço de movimentação. A mobilidade corresponde geralmente ao número mínimo de atuadores necessários para a movimentação do órgão terminal.

Para o cálculo da mobilidade utilizamos o critério de Kutzbach-Gruebler[6]:

$$
M=\lambda(n-1)-\sum_{i=1}^{\lambda-1}(\lambda-i) n_{j i}
$$

Onde $M$ é a mobilidade, $\lambda$ é o número de movimentos independentes que uma peça pode executar num determinado espaço sem vínculo $(\lambda=3$ se for mecanismo plano e $\lambda=6$ para mecanismo tridimensional), $n$ é o número de peças do mecanismo incluindo a base fixa e $n_{j i}$ é o número de juntas com grau de liberdade $i$.

Então para o mecanismo plano temos:

$$
M=3(n-1)-2 n_{j 1}-n_{j 2}
$$

No caso do mecanismo $2 \underline{R} R R+R P$ objeto de nosso trabalho, temos $n=7$, $\lambda=3, n_{j 1}=8$ e $n_{j 2}=0$ donde concluímos que sua mobilidade é 2 . 


\subsection{Análise cinemática}

A cinemática estuda o movimento dos corpos independentemente de suas causas. Com a utilização da análise cinemática podemos avaliar deslocamentos, velocidades e acelerações dos componentes de um mecanismo. Estas avaliações serão importantes para o desenvolvimento da análise dinâmica.

A análise cinemática pode ser direta ou inversa. Na cinemática direta são conhecidas as coordenadas das juntas ativas e propomos determinar as coordenadas do órgão terminal. Na cinemática inversa conhecemos a posição do órgão terminal e propomos determinar as coordenadas das juntas ativas.

O problema da cinemática inversa é que poderão existir inúmeras soluções para uma determinada posição do órgão terminal, criando a necessidade de estabelecermos outros critérios para a definição de um resultado final não disperso.

Ao desenvolvermos as equações da cinemática inversa verificaremos que as mesmas são não-lineares e independentes, enquanto que na cinemática direta as suas equações são também não-lineares, todavia dependentes, o que torna a cinemática direta mais complexa[7]. Enquanto que no caso dos mecanismos paralelos a solução da cinemática direta é mais difícil que a da cinemática inversa, no caso dos mecanismos seriais ocorre o contrário.

\subsection{Espaço de trabalho}

O espaço de trabalho basicamente é o espaço no qual o órgão terminal do mecanismo pode alcançar[8]. A análise do espaço de trabalho de um mecanismo é muito importante pois é através dela que podemos determinar sua capacidade de movimentação e, em conseqüência, os limites de sua aplicação[9][10].

Merlet faz algumas ponderações interessantes sobre o espaço de trabalho em[11]:

"A principal dificuldade da análise do espaço de trabalho para mecanismos paralelos é que, como as posições alcançáveis pelo órgão terminal dependem de sua orientação, uma representação completa do espaço de trabalho deve ser englobada num espaço de 6 dimensões para o qual não é possível uma ilustração gráfica. Somente subconjuntos do espaço de trabalho podem, conseqüentemente, ser representados."

No caso de mecanismos seriais de 6 graus de liberdade o espaço de trabalho é normalmente representado pelo espaço alcançável pelo centro do pulso, isto é, pelo espaço alcançado pelas translações do centro do pulso. Os três últimos graus de liberdade apenas orientam o órgão terminal. 
No caso de mecanismos paralelos não podemos fazer esta distinção pois as translações dependem da rotação do órgão terminal.

Face ao exposto, o espaço de trabalho para mecanismos paralelos pode ser classificado em:

1. Espaço de trabalho de translação ( ou espaço de trabalho de orientação constante) é o espaço definido pelo conjunto de pontos que podem ser alcançados pelo ponto de referência do órgão terminal $\left(P_{R}\right)$ quando a orientação do órgão terminal é fixa. É o mais utilizado e na sua determinação podemos usar tanto a abordagem geométrica como a algébrica onde a primeira é mais rápida e a última mais geral[11].

2. Espaço de trabalho de orientação é o espaço definido pelo conjunto de pontos que podem ser alcançados por $P_{R}$ com as possíveis rotações do órgão terminal em torno de um ponto nele situado[12].

Outros tipos de espaço de trabalho também podem ser de interesse como o espaço de trabalho alcançável (todas as localizações que podem ser alcançadas por $P_{R}$ com pelo menos uma orientação do órgão terminal), espaço de trabalho de orientação total (localizações alcançadas por $P_{R}$ com todas as orientações pertencentes a um dado conjunto de orientações).

A movimentação dos mecanismos paralelos está sujeita a várias restrições como limitações mecânicas das juntas passivas, limitações próprias dos atuadores, possibilidades de interferência entre suas partes móveis além, logicamente, de suas limitações dimensionais.

A determinação do espaço de trabalho pode ser feita por vários métodos: o geométrico, por discretização e utilizando-se a otimização.

\subsection{Análise dinâmica}

Num mecanismo atuam forças e momentos internos e externos. Dentre as forças e momentos externos citamos as forças gravitacionais, as forças originadas pelo processo de fabricação (como, por exemplo, as forças de usinagem) as forças e torques dos atuadores, etc.

No que se refere a forças e momentos internos podemos citar aqueles que agem nas articulações do mecanismo, os efeitos das propriedades de distribuição (massa, momento de inércia, baricentro), os efeitos das propriedades cinemáticas (comprimento da barra, posição relativa, etc.), o efeito do atrito, etc. Os efeitos dos esforços de deformações e vibrações não serão considerados neste trabalho.

A grande finalidade da análise dinâmica é, conhecendo-se as forças e momen- 
tos aplicados no mecanismo, podermos calcular as forças e momentos internos para que se possa dimensionar adequadamente as sua peças. Outra finalidade é a geração do modelo dinâmico para ser utilizado no sistema de controle do mecanismo.

Semelhantemente à análise cinemática a análise dinâmica pode ser apresentada de duas formas: dinâmica inversa e dinâmica direta. Na dinâmica inversa o movimento do mecanismo é conhecido e busca-se determinar as força e momentos nos atuadores. Na dinâmica direta são conhecidos as forças e momentos dos atuadores e busca-se determinar seu movimento.

Neste trabalho só trataremos da dinâmica inversa e com a imposição dos corpos serem rígidos.

\subsection{Métodos para análise dinâmica}

Existem vários métodos para resolver a análise dinâmica e dentre eles destacamos o de Newton-Euler e o de Lagrange. Em nosso trabalho, entretanto, empregaremos um outro método mais recente e menos conhecido mas muito eficiente: o método de Kane.

\subsubsection{Método de Newton-Euler}

É um método que relaciona forças e momentos. Quando o movimento é composto de translação e rotação empregamos o Teorema do Movimento do Baricentro (TMB) para resolver a parte da translação e a parte rotação é resolvida pelo Teorema do Momento Angular (TMA).

Através deste método é possível calcular os esforços de reação em todas as peças do mecanismo e, portanto, é um método muito bom quando se precisa dimensionar as suas peças. A aplicação do método é relativamente fácil, mas sua resolução é trabalhosa, pois as equações originadas por este método são complexas e em grande número, logo sua eficiência computacional é baixa.

As fórmulas de Newton-Euler para a solução de um sistema de $n$ corpos são:

$$
\begin{gathered}
\vec{R}_{i}=m_{i} \vec{A}_{i} \\
\vec{T}_{i}=I_{i} \dot{\vec{w}_{i}}+\vec{w}_{i} \wedge I_{i} \vec{w}_{i} \quad(i=1, \ldots, n)
\end{gathered}
$$

Sendo,

$\vec{R}_{i}$ : resultante das forças externas atuantes no corpo $i$ 
$m_{i}$ : massa do corpo $i$

$\vec{A}_{i}$ : aceleração do centro de massa do corpo $i$

$\vec{T}_{i}$ : momento resultante dos momentos externos atuantes no corpo $i$

$I_{i}$ : momento de inércia do corpo $i$ em relação ao seu eixo baricêntrico

$\overrightarrow{w_{i}}$ e $\dot{w_{i}}$ : velocidade angular do centro de massa do corpo $i$ e sua derivada temporal.

\subsubsection{Método de Lagrange}

Este método leva em conta a energia cinética e potencial de cada peça. Ele faz a avaliação utilizando as chamadas coordenadas generalizadas (geralmente representadas por $q_{k}$ ) que correspondem aos graus de liberdade do sistema. Como este método elimina os esforços internos a sua eficiência computacional é ótima e, portanto, mais interessante para sistemas de controle em tempo real. Porém a dedução das equações com coordenadas generalizadas independentes é muito difícil, exigindo às vezes, a adição de coordenadas extras. Além disso, como este método lida com energia, não transparece o discernimento físico que o método de Newton-Euler apresenta.

A fórmula de Lagrange é a seguinte:

$$
\frac{d}{d t}\left(\frac{\partial L}{\partial \dot{q}_{k}}\right)-\frac{\partial L}{\partial q_{k}}=Q_{k} \quad(k=1, \ldots, M)
$$

Sendo $L$ a lagrangeana, isto é, a diferença entre a energia cinética e potencial do sistema, $q_{k}$ é a coordenada generalizada, $M$ é a quantidade de graus de liberdade, ou seja, é a mobilidade do mecanismo e $Q_{k}$ é a força generalizada.

\subsubsection{Método de Kane}

O método de Kane em seu início também relaciona forças e momentos e utiliza o TMB e o TMA como no método de Newton-Euler mas depois deve-se especificar as variáveis de movimento, normalmente representadas por $u_{r}$. As equações de Kane são deduzidas a partir do produto escalar das equações obtidas pelo método de Newton-Euler (ou equações do TMB e TMA), pelas derivadas parciais, respectivamente das velocidades lineares e angulares dos centros de gravidade de cada peça, em relação às variáveis de movimento. Com isto obtemos equações escalares que deverão ser reunidas membro a membro, respectivamente para cada par de peças conectadas e para cada índice $r$ da variável de movimento, obtendo-se um conjunto final de equações. A quantidade destas equações normalmente coincide 
com a mobilidade do mecanismo, o que dá uma idéia da redução do número de equações ao se comparar com o método de Newton-Euler.

Por este método é possível também calcular os esforços de reação em todas as peças do mecanismo. O método de Kane apresenta uma sistemática de solução bem estruturada e, além disso, oferece bom discernimento físico por lidar com forças e momentos. Entretanto tem a desvantagem em primeiro lugar de ser pouco conhecido e depois pela dificuldade da determinação das variáveis de movimento.

As fórmulas empregadas no método de Kane são:

$$
F_{r}+F_{r}^{*}=0 \quad(r=1, \ldots, M)
$$

Onde

$$
\begin{gathered}
F_{r}=\sum_{i=1}^{n}\left(\overrightarrow{R_{i}} \cdot \frac{\partial \overrightarrow{v_{i}}}{\partial u_{r}}+\overrightarrow{T_{i}} \cdot \frac{\partial \vec{w}_{i}}{\partial u_{r}}\right) \\
F_{r}^{*}=-\sum_{i=1}^{n}\left(m_{i} \overrightarrow{A_{i}} \cdot \frac{\partial \overrightarrow{v_{i}}}{\partial u_{r}}+\left(I_{i} \overrightarrow{\alpha_{i}}+\overrightarrow{w_{i}} \wedge I_{k} \overrightarrow{w_{i}}\right) \cdot \frac{\partial \overrightarrow{w_{i}}}{\partial u_{r}}\right)
\end{gathered}
$$

Sendo

$\overrightarrow{v_{i}}$ : velocidade linear do centro de massa do corpo $i$

$\overrightarrow{\alpha_{i}}=\overrightarrow{w_{i}}$ : aceleração angular do centro de massa do corpo $i$

$u_{r}$ : variável de movimento de Kane

$M$ : quantidade de variáveis de movimento de Kane

$n$ : quantidade de corpos do sistema

Demais símbolos são iguais aos da seção Método de Newton-Euler acima.

\subsection{Síntese. Método da adição de uma cadeia passiva.}

Existem vários métodos para a geração da arquitetura de mecanismos paralelos como, por exemplo, o método da enumeração das cadeias ativas, o método da adição de uma cadeia passiva, etc.

No método da adição de uma cadeia passiva o movimento da plataforma móvel é restringido pela cadeia passiva. Assim sendo, se quisermos um mecanismo com uma determinada mobilidade $M$ (ou grau de mobilidade $G M$ ) devemos criar uma cadeia passiva que possua uma conectividade correspondente à esta mobilidade.

A conectividade de uma cadeia é determinada pela soma dos graus de liberdade $(G L)$ das juntas existentes nesta cadeia. Chamando de $C_{n+1}$ à conectividade 
da cadeia passiva podemos então colocar

$$
C_{n+1}=M
$$

As conectividades $C_{k}$ ( sendo $k=1, \ldots, m$ ) das $m$ cadeias ativas do mecanismo devem ser iguais ao número de movimentos independentes $\lambda$ que o mecanismo pode executar no seu espaço de funcionamento, isto é, $\lambda=3$ para o espaço plano e $\lambda=6$ para o espaço tridimensional, então temos

$$
C_{k}=\lambda \quad(k=1, \ldots, m)
$$

O número de cadeias ativas deve ser igual à mobilidade do mecanismo, donde

$$
m=M
$$

O manipulador robótico paralelo espacial de $3 G M$ para operações Pick-AndPlace, $3 \underline{R} U S+C P$, mostrado na fig. 2.4 e que serviu de base para o mecanismo plano objeto desta dissertação, foi criado à partir de uma cadeia passiva que permite translação e rotação da plataforma móvel. A conectividade desta cadeia passiva é $C_{n+1}=3$ pois a mesma é composta de uma junta cilíndrica $(G L=2)$ e de uma junta prismática $(G L=1)$. Portanto a mobilidade $M$ do mecanismo é 3 e em conseqüência o número $m$ de cadeias ativas deve ser 3 .

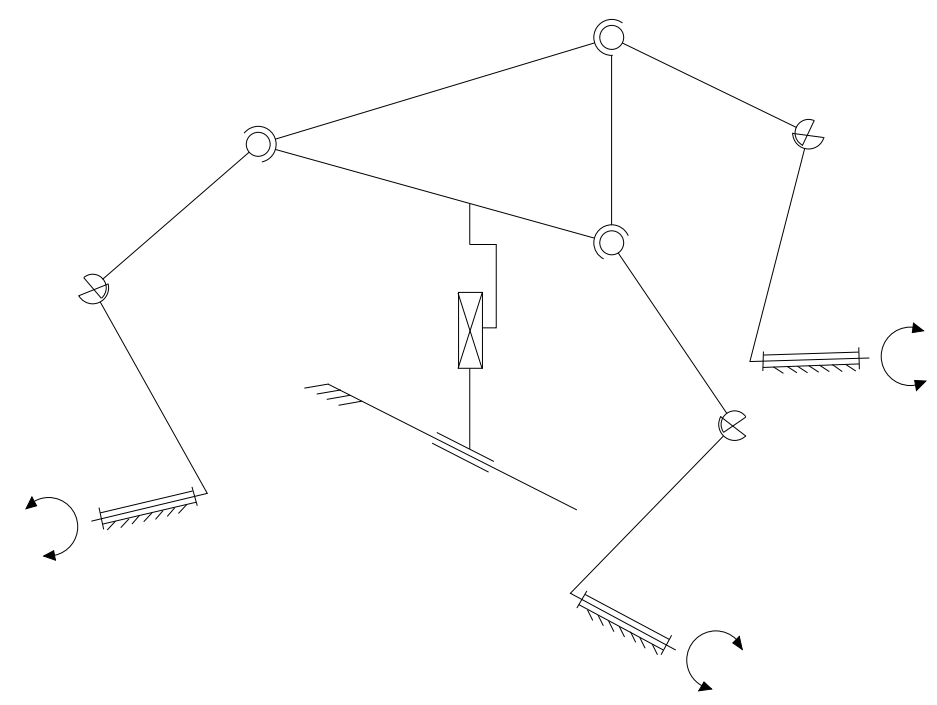

Figura 2.4: Diagrama cinemático do manipulador robótico paralelo espacial 3GM

Como $\lambda=6$, o mecanismo deverá possuir 3 cadeias ativas com conectividade 
igual a 6 e no nosso caso foram escolhidas as cadeias ativas compostas de uma junta de rotação com atuador $(\underline{R})$, uma junta universal $(U)$ e uma junta esférica $(S)$.

\subsection{Metodologia empregada}

A metodologia empregada nesta pesquisa seguirá a seqüência mostrada no fluxograma da fig.2.5. Neste procuramos mostrar os respectivos dados de entrada e resultados a serem obtidos em cada etapa. Para cada etapa elaboramos um programa de cálculo desenvolvido através do software Mathematica, os quais estão mostrados nos Anexos, para serem utilizados nas aplicações numéricas.

Tomamos como ponto de partida o mecanismo paralelo plano $2 \underline{R} R R+R P$, conforme já descrito anteriormente. Admitiremos como conhecidos todos os seus parâmetros: comprimento das barras, massas, etc.

Análise cinemática: posições. Nesta análise relacionamos as coordenadas dos atuadores com as coordenadas da garra. Fornecendo-se os parâmetros do mecanismo, na cinemática inversa obteremos as coordenadas dos atuadores e na cinemática direta obteremos as coordenadas da garra. Estes resultados serão utilizados em primeiro lugar na verificação se o espaço de trabalho do mecanismo atende as posições impostas; em segundo lugar na Análise cinemática: velocidades e acelerações; em terceiro lugar nas Análises dinâmicas; e em quarto lugar no Efeito do atrito.

Espaço de trabalho. Nesta etapa tendo-se os parâmetros podemos verificar se o espaço de trabalho atende as posições requeridas do mecanismo.

Trajetória da garra. Nesta etapa devemos fornecer os dados de projeto referentes à velocidade máxima, aceleração e desaceleração e tipo de trajetória para obtermos como resultados a velocidade e aceleração finais da garra. Estes resultados serão utilizados nas etapas Analises dinâmicas I e II, e Efeito do atrito.

Análise cinemática: velocidades e acelerações. Nesta etapa devemos fornecer os parâmetros, os resultados obtidos na Análise cinemática: posições (ângulos dos elos) e os resultados obtidos na etapa Trajetória da garra (velocidade e aceleração da garra). Com a entrada destes dados teremos como resultados as velocidades e acelerações angulares dos elos e as acelerações dos baricentros (CG) dos elos, os quais serão utilizados posteriormente nas etapas Análises dinâmicas I e II, e Efeito do atrito.

Análise estática. Nesta etapa devemos fornecer os pesos dos elos e dos contrapesos, as posições dos seus CG, força e momento atuantes na garra, etc. e 
obteremos como resultados as reações estáticas.

Análise dinâmica I. Nesta etapa devemos fornecer os parâmetros mais os resultados das etapas Análise cinemática: posições, Trajetória da garra, Análise cinemática: velocidades e acelerações, aceleração da gravidade e obteremos as reações dinâmicas sem levar em conta os esforços externos na garra e o atrito.

Análise dinâmica II. Nesta etapa devemos fornecer os mesmos dados da Análise dinâmica I mais os resultados da Análise estática e obteremos as reações dinâmicas, levando em conta os esforços externos na garra mas sem considerar o atrito.

Efeito do atrito. Nesta última etapa devemos fornecer os mesmos dados da Análise dinâmica I, com os esforços externos atuantes na garra e os dados de atrito. Como resultado obteremos as reações dinâmicas levando em conta os esforços na garra e o atrito. 


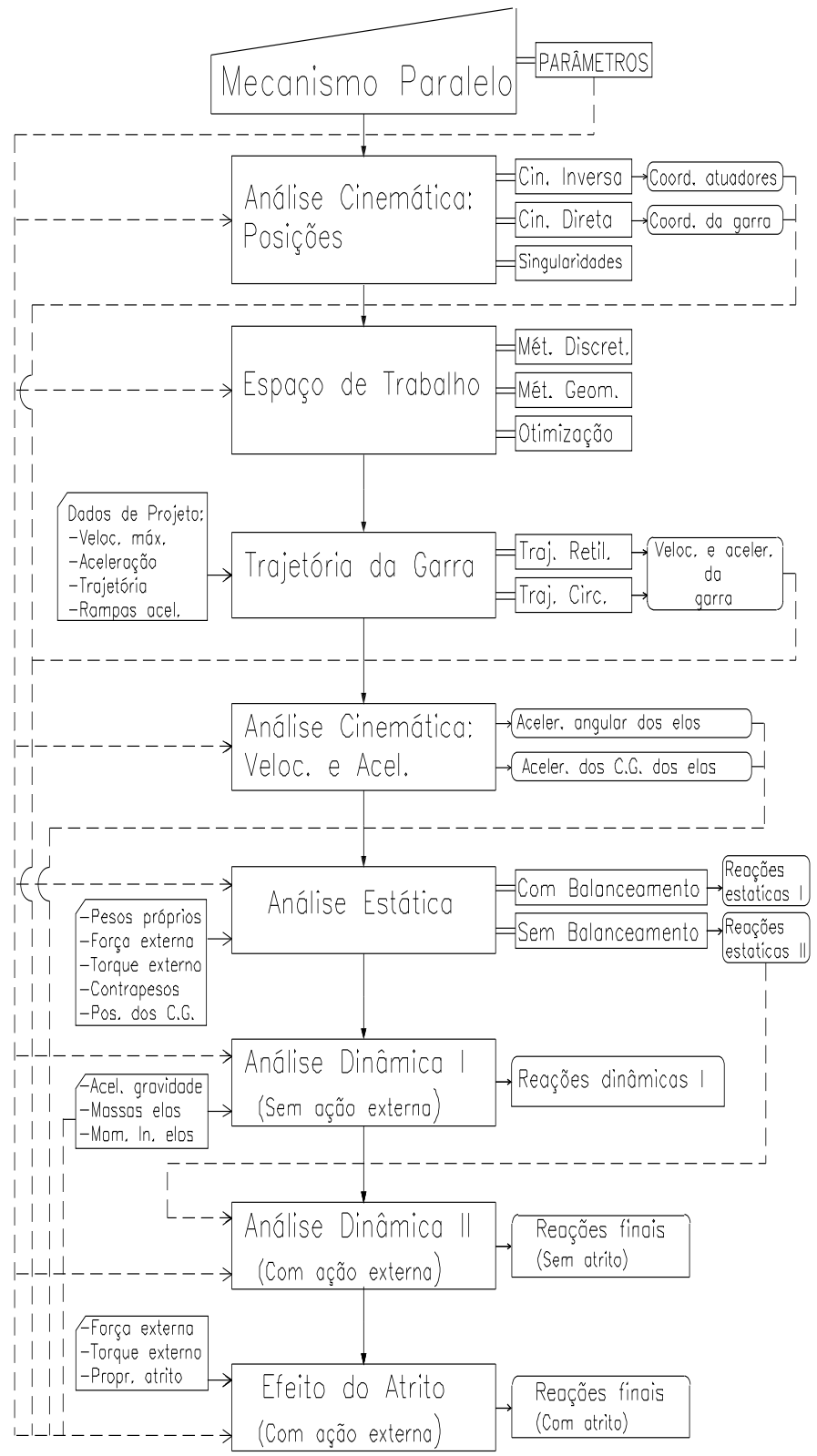

Figura 2.5: Fluxograma 


\section{Capítulo 3: ANÁLISE CINEMÁTICA: POSIÇÕES}

\subsection{Cinemática inversa}

Nosso objetivo nesta análise é obtermos uma relação matemática entre a posição da garra (ponto $G$ ) com as coordenadas dos atuadores. Isto será muito importante no estudo do espaço de trabalho, trajetórias, análise estática, etc.

Vamos chamar de $L_{n}$ o comprimento do elo $n$ e de $\theta_{n}$ o ângulo que ele forma com a horizontal e vamos adotar as seguintes simplificações de notação: $\operatorname{sen} \theta_{n}=$ $s \theta_{n}=s_{n}$ e $\cos \theta_{n}=c \theta_{n}=c_{n}$.

A Fig.3.1 apresenta o diagrama cinemático do mecanismo com as coordenadas dos seus vértices e os ângulos dos elos com a horizontal.

Na cinemática inversa $L_{7}$ e $\theta_{7}$ são conhecidos, e $\theta_{2}$ e $\theta_{6}$ são incógnitas. Nosso objetivo, portanto, é determinar estes ângulos.

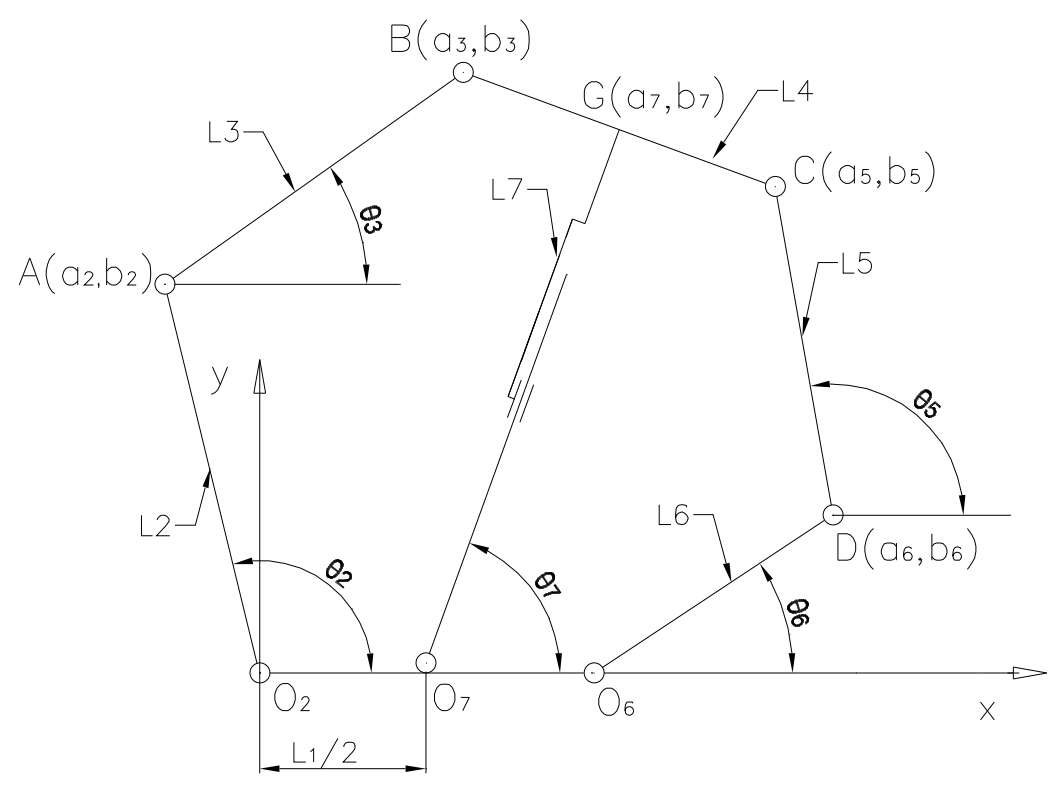

Figura 3.1: Mecanismo: coordenada dos vértices.

\section{Coordenadas dos pontos}

Em primeiro lugar vamos determinar as coordenadas dos pontos $G, B$ e $C$. 


$$
\begin{aligned}
G: & \left\{a_{7}, b_{7}\right\}=\left\{\frac{L_{1}}{2}+L_{7} c_{7}, L_{7} s_{7}\right\} \\
B: & \left\{a_{3}, b_{3}\right\}=\frac{L_{4}}{2}\left\{-s_{7}, c_{7}\right\}+\left\{a_{7}, b_{7}\right\} \\
C: \quad & \left\{a_{5}, b_{5}\right\}=\frac{L_{4}}{2}\left\{s_{7},-c_{7}\right\}+\left\{a_{7}, b_{7}\right\}
\end{aligned}
$$

O ponto A é a interseção das circunferências com centro em $O_{2}$ e raio $L_{2}$ e centro em B e raio $L_{3}$ :

$$
\begin{gathered}
x^{2}+y^{2}=L_{2}^{2} \\
\left(x-a_{3}\right)^{2}+\left(y-b_{3}\right)^{2}=L_{3}^{2}
\end{gathered}
$$

Resolvendo estas duas equações em $x$ e $y$ obteremos as coordenadas do ponto $A\left(a_{2}, b_{2}\right)$.

O ponto D é a interseção das circunferências com centro em $O_{6}$ e raio $L_{6}$ e centro em $C$ e raio $L_{5}$ :

$$
\begin{aligned}
\left(x-L_{1}\right)^{2}+y^{2} & =L_{6}^{2} \\
\left(x-a_{5}\right)^{2}+\left(y-b_{5}\right)^{2} & =L_{5}^{2}
\end{aligned}
$$

Com estas equações determinamos $D\left(a_{6}, b_{6}\right)$.

Mas temos duas soluções para cada vértice $A$ e $D$. Ou seja, para o vértice $A$ temos como soluções os pontos $A^{\prime}$ e $A^{\prime \prime}$ que são simétricos em relação à reta $\mathrm{O}_{2} B$. Do mesmo modo, quanto ao vértice $D$ temos as soluções $D^{\prime}$ e $D^{\prime \prime}$ que são simétricos em relação à reta $O_{6} C$. No programa que fizemos no Anexo A impomos as condições de $A$ estar sempre à esquerda do segmento $O_{2} B$ e $D$ à direita de $O_{6} C$, utilizando a propriedade do produto vetorial (ver mais detalhes no Capítulo 10, seção 10.1.1).

\section{Conclusão}

No Anexo A apresentamos o programa do software Mathematica para solução da cinemática inversa

Fornecendo-se os seguintes dados:

- Comprimento dos elos: $L_{1}$ a $L_{6}$.

- Comprimento da cadeia passiva e seu ângulo de inclinação: $L_{7}$ e $\theta_{7}$. 
O programa determina:

- Os ângulos de entrada: $\theta_{2}$ e $\theta_{6}$.

- Os ângulos dos elos 3 e $5: \theta_{3}$ e $\theta_{5}$.

- Verifica a existência de singularidades e informa seus tipos (conforme desenvolvido na seção adiante).

- Traça o gráfico do mecanismo

\section{Exemplo de aplicação}

A tabela 3.1 foi feita considerando um mecanismo onde todos os lados têm comprimento unitário $\left(L_{1}=L_{2}=\ldots .=L_{7}=1\right)$ e variamos o ângulo da cadeia passiva $\theta_{7}$ para ver qual é a variação dos ângulos $\theta_{2}, \theta_{3}, \theta_{5}$ e $\theta_{6}$.

\begin{tabular}{cccccc}
\hline$\theta_{7}$ & $90^{\circ}$ & $75^{\circ}$ & $60^{\circ}$ & $45^{\circ}$ & $15^{\circ}$ \\
\hline$\theta_{2}$ & $150^{\circ}$ & $131,5^{\circ}$ & $114,3^{\circ}$ & $98,3^{\circ}$ & $69^{\circ}$ \\
$\theta_{3}$ & $30^{\circ}$ & $20,25^{\circ}$ & $11,8^{\circ}$ & $4,07^{\circ}$ & $348^{\circ}$ \\
$\theta_{5}$ & $150^{\circ}$ & $138^{\circ}$ & $122,8^{\circ}$ & $102,9^{\circ}$ & $50^{\circ}$ \\
$\theta_{6}$ & $30^{\circ}$ & $9,7^{\circ}$ & $-13^{\circ}$ & $-38,4^{\circ}$ & $268^{\circ}$ \\
\hline
\end{tabular}

Tabela 3.1: Exemplo cinemática inversa.

\subsection{Cinemática direta}

Neste caso são dados os ângulos de entrada $\theta_{2}$ e $\theta_{6}$ e vamos determinar $L_{7}$ e $\theta_{7}$.

Coordenadas dos pontos $A$ e $D$ :

$$
\begin{aligned}
A: & \left\{a_{2}, b_{2}\right\}=\left\{L_{2} c_{2}, L_{2} s_{2}\right\} \\
D: & \left\{a_{6}, b_{6}\right\}=\left\{L_{1}+L_{6} c_{6}, L_{6} s_{6}\right\}
\end{aligned}
$$

As coordenadas de $B$ e $C$ serão calculadas em função de $L_{7}$ e $\theta_{7}$ :

$$
\begin{array}{rlrl}
B: & \left\{a_{3}, b_{3}\right\} & =\left\{\frac{L_{1}}{2}, 0\right\}+L_{7}\left\{c_{7}, s_{7}\right\}+\frac{L_{4}}{2}\left\{-s_{7}, c_{7}\right\} \\
C: & \left\{a_{5}, b_{5}\right\}=\left\{\frac{L_{1}}{2}, 0\right\}+L_{7}\left\{c_{7}, s_{7}\right\}+\frac{L_{4}}{2}\left\{s_{7},-c_{7}\right\}
\end{array}
$$


Mas como

$$
s_{7}^{2}+c_{7}^{2}=1
$$

então

$$
s_{7}=\sqrt{1-c_{7}^{2}} .
$$

E $s_{7}$ é sempre positivo porque $\theta_{7} \in[0, \pi]$, pois uma das condições que estamos impondo ao mecanismo é que a cadeia passiva nunca avance para baixo da base $L_{1}$. Então as coordenadas dos pontos $B$ e $C$ podem ser escritas assim:

$$
\begin{aligned}
B: & \left\{a_{3}, b_{3}\right\}=\left\{\frac{L_{1}}{2}, 0\right\}+L_{7}\left\{c_{7}, \sqrt{1-c_{7}^{2}}\right\}+\frac{L_{4}}{2}\left\{-\sqrt{1-c_{7}^{2}}, c_{7}\right\}, \\
C: & \left\{a_{5}, b_{5}\right\}=\left\{\frac{L_{1}}{2}, 0\right\}+L_{7}\left\{c_{7}, \sqrt{1-c_{7}^{2}}\right\}+\frac{L_{4}}{2}\left\{\sqrt{1-c_{7}^{2}},-c_{7}\right\} .
\end{aligned}
$$

Por outro lado, como a distância entre $A$ e $B$ é $L_{3}$ e entre $C$ e $D$ é $L_{5}$, podemos escrever:

$$
\begin{aligned}
& \left(a_{2}-a_{3}\right)^{2}+\left(b_{2}-b_{3}\right)^{2}=L_{3}^{2}, \\
& \left(a_{5}-a_{6}\right)^{2}+\left(b_{5}-b_{6}\right)^{2}=L_{5}^{2} .
\end{aligned}
$$

Com estas equações podemos calcular os valores de $a_{3}, b_{3}, a_{5}, b_{5}, L_{7}$ e $\theta_{7}$.

No Anexo B apresentamos o programa do software Mathematica para solução da cinemática direta onde consideramos as seguintes condições:

- $L_{7} \geqslant 0$

- $0 \leqslant \theta_{7} \leqslant \pi$

- A plataforma nunca deve cruzar com a base $L_{1}$ ou com os elos inferiores $L_{2}$ e $L_{6}$ (ver mais detalhes no Capítulo 10, seção 10.1.2).

Fornecendo-se o comprimento dos elos $L_{1}$ a $L_{6}$ e os ângulos de entrada $\theta_{2}$ e $\theta_{6}$, o programa calcula o comprimento da cadeia passiva $L_{7}$ e seu ângulo de inclinação $\theta_{7}$, e mostra um gráfico do mecanismo.

\subsection{Singularidades}

\subsubsection{Introdução}

Segundo Merlet [4] as singularidades são posições particulares nas quais o mecanismo perde sua rigidez e o órgão terminal passa a apresentar graus de liberdade adicionais tornando-se incontrolável. Existem outras situações nas quais o 
mecanismo pode perder graus de liberdade quando, por exemplo, a plataforma móvel se aproxima dos limites de seu espaço de trabalho.

Uma maneira de detectar as singularidades é através da matriz jacobiana. Se o determinante da matriz jacobiana é zero, o sistema de equações, que transforma as forças aplicadas na plataforma móvel em forças nos atuadores, não pode mais ser resolvido. Consequentemente ou a rigidez da plataforma móvel se anula em alguma direção ou o esforço em uma das juntas é infinito [13]. Conforme Gosselin e Angeles [14] a matriz jacobiana pode ser separada em uma matriz que está associada à cinemática inversa e outra que está associada à cinemática direta. Exemplos de aplicação poderão ser vistos em [15] e [16].

Chamando de $\vec{x}$ o vetor das coordenadas do órgão terminal e de $\vec{q}$ o vetor das coordenadas dos atuadores, podemos relacioná-los através da equação:

$$
f(\vec{x}, \vec{q})=\overrightarrow{0}
$$

Diferenciando-se esta equação em relação ao tempo e colocando o resultado na forma matricial, temos:

$$
J_{x} \dot{\vec{x}}+J_{q} \dot{\vec{q}}=\overrightarrow{0}
$$

Nesta equação $J_{x}$ e $J_{q}$ são as matrizes jacobianas; $\dot{\vec{x}}$ e $\dot{\vec{q}}$ são respectivamente os vetores das velocidades do órgão terminal e dos atuadores.

Se $\operatorname{det} J_{x}=0$ temos a singularidade direta. Neste caso o órgão terminal adquire graus de liberdade adicionais, isto é, o órgão terminal pode se movimentar mesmo com os atuadores parados. É um tipo de singularidade que ocorre no interior do espaço de trabalho.

Se $\operatorname{det} J_{q}=0$ temos a singularidade inversa. Neste caso o órgão terminal perde graus de liberdade, isto é, o órgão terminal não pode se movimentar em determinadas direções. Este tipo de singularidade ocorre nas fronteiras do espaço de trabalho.

Quando tanto det $J_{x}$ quanto $\operatorname{det} J_{q}$ são nulos temos as singularidades combinadas.

\subsubsection{Determinação das matrizes jacobianas}

Considerando a cadeia $O_{2} A B$ podemos escrever:

$$
\left(a_{2}-a_{3}\right)^{2}+\left(b_{2}-b_{3}\right)^{2}=L_{3}^{2}
$$


Isto é,

$$
\begin{gathered}
L_{2}^{2}+\left(L_{7} c_{7}-\frac{L_{4} s_{7}}{2}+\frac{L_{1}}{2}\right)^{2}-2\left(L_{7} c_{7}-\frac{L_{4} s_{7}}{2}+\frac{L_{1}}{2}\right) L_{2} c_{2}+ \\
\left(L_{7} s_{7}+\frac{L_{4} c_{7}}{2}\right)^{2}-2\left(L_{7} s_{7}+\frac{L_{4} c_{7}}{2}\right) L_{2} s_{2}=L_{3}^{2}
\end{gathered}
$$

Derivando em relação ao tempo e rearranjando chegamos a

$$
\left(-2 L_{2} c_{2} c_{7}+L_{1} c_{7}-2 L_{2} s_{2} s_{7}+2 L_{7}\right) \dot{L_{7}}+
$$

$\left(2 L_{2} L_{7} c_{2} s_{7}+L_{2} L_{4} s_{2} s_{7}-L_{1} L_{7} s_{7}+L_{2} L_{4} c_{2} c_{7}-2 L_{2} L_{7} s_{2} c_{7}-L_{1} L_{4} c_{7} / 2\right) \dot{\theta}_{7}+$

$$
\left(2 L_{2} L_{7} s_{2} c_{7}-L_{2} L_{4} c_{2} c_{7}-L_{2} L_{4} s_{2} s_{7}-2 L_{2} L_{7} c_{2} s_{7}-L_{1} L_{2} s_{2}\right) \dot{\theta_{2}}=0
$$

Colocando

$$
\left(-2 L_{2} c_{2} c_{7}+L_{1} c_{7}-2 L_{2} s_{2} s_{7}+2 L_{7}\right)=p_{11}
$$

$\left(2 L_{2} L_{7} c_{2} s_{7}+L_{2} L_{4} s_{2} s_{7}-L_{1} L_{7} s_{7}+L_{2} L_{4} c_{2} c_{7}-2 L_{2} L_{7} s_{2} c_{7}-L_{1} L_{4} c_{7} / 2\right)=p_{12}$

$\left(2 L_{2} L_{7} s_{2} c_{7}-L_{2} L_{4} c_{2} c_{7}-L_{2} L_{4} s_{2} s_{7}-2 L_{2} L_{7} c_{2} s_{7}-L_{1} L_{2} s_{2}\right)=q_{11}$

as equações acima podem ser escritas assim

$$
p_{11} \dot{L}_{7}+p_{12} \dot{\theta}_{7}+q_{11} \dot{\theta}_{2}=0
$$

Considerando agora a cadeia $\mathrm{O}_{6} \mathrm{DC}$ temos:

$$
\left(a_{5}-a_{6}\right)^{2}+\left(b_{5}-b_{6}\right)^{2}=L_{5}^{2}
$$

Isto é,

$$
\begin{gathered}
\left(L_{7} c_{7}+\frac{L_{4} s_{7}}{2}-\frac{L_{1}}{2}\right)^{2}-2\left(L_{7} c_{7}+\frac{L_{4} s_{7}}{2}-\frac{L_{1}}{2}\right) L_{6} c_{6}+\left(L_{7} s_{7}-\frac{L_{4} c_{7}}{2}\right)^{2}- \\
2\left(L_{7} s_{7}-\frac{L_{4} c_{7}}{2}\right) L_{6} s_{6}+L_{6}^{2}=L_{5}^{2}
\end{gathered}
$$

Derivando em relação ao tempo e rearranjando chegamos a

$$
\begin{gathered}
\left(-2 L_{6} c_{6} c_{7}-L_{1} c_{7}-2 L_{6} s_{6} s_{7}+2 L_{7}\right) \dot{L}_{7}+ \\
\left(2 L_{6} L_{7} c_{6} s_{7}-L_{6} L_{4} s_{6} s_{7}+L_{1} L_{7} s_{7}-L_{6} L_{4} c_{6} c_{7}-2 L_{6} L_{7} s_{6} c_{7}-L_{1} L_{4} c_{7} / 2\right) \dot{\theta}_{7}+
\end{gathered}
$$




$$
\left(2 L_{6} L_{7} s_{6} c_{7}+L_{6} L_{4} c_{6} c_{7}+L_{6} L_{4} s_{6} s_{7}-2 L_{6} L_{7} c_{6} s_{7}-L_{1} L_{6} s_{6}\right) \dot{\theta_{6}}=0
$$

Colocando

$$
\left(-2 L_{6} c_{6} c_{7}-L_{1} c_{7}-2 L_{6} s_{6} s_{7}+2 L_{7}\right)=p_{21}
$$

$\left(2 L_{6} L_{7} c_{6} s_{7}-L_{6} L_{4} s_{6} s_{7}+L_{1} L_{7} s_{7}-L_{6} L_{4} c_{6} c_{7}-2 L_{6} L_{7} s_{6} c_{7}-L_{1} L_{4} c_{7} / 2\right)=p_{22}$

$$
\left(2 L_{6} L_{7} s_{6} c_{7}+L_{6} L_{4} c_{6} c_{7}+L_{6} L_{4} s_{6} s_{7}-2 L_{6} L_{7} c_{6} s_{7}-L_{1} L_{6} s_{6}\right)=q_{22}
$$

as equações acima podem ser escritas assim

$$
p_{21} \dot{L}_{7}+p_{22} \dot{\theta}_{7}+q_{22} \dot{\theta}_{6}=0
$$

As equações 3.8 e 3.9 podem ser escritas na forma matricial

$$
\left[\begin{array}{ll}
p_{11} & p_{12} \\
p_{21} & p_{22}
\end{array}\right]\left[\begin{array}{c}
\dot{L}_{7} \\
\dot{\theta}_{7}
\end{array}\right]+\left[\begin{array}{cc}
q_{11} & 0 \\
0 & q_{22}
\end{array}\right]\left[\begin{array}{c}
\dot{\theta}_{2} \\
\dot{\theta}_{6}
\end{array}\right]=0
$$

Então as matrizes jacobianas são

$$
J_{x}=\left[\begin{array}{ll}
p_{11} & p_{12} \\
p_{21} & p_{22}
\end{array}\right]
$$

$\mathrm{e}$

$$
J_{q}=\left[\begin{array}{cc}
q_{11} & 0 \\
0 & q_{22}
\end{array}\right]
$$

No Anexo A utilizamos estas matrizes jacobianas para a determinação das singularidades. 


\section{Capítulo 4: ESTUDO DO ESPAÇO DE TRABALHO}

\subsection{Método por discretização}

O método por discretização para determinação do espaço de trabalho é um método que desenvolve um domínio (um reticulado) de pontos com espaçamentos previamente definidos e que devem satisfazer todas as restrições impostas.

Podemos definir o nosso espaço de trabalho como o lugar geométrico que o ponto $G$ (ponto de referência da garra do mecanismo) poderá atingir quando a plataforma móvel é movimentada para qualquer posição que o mecanismo permita, limitado inferiormente pela reta que passa pelos pontos $\mathrm{O}_{2}$ e $\mathrm{O}_{6}$. Portanto conforme a fig. 4.1 a primeira restrição que estamos impondo é:

$$
0 \leqslant \theta_{7} \leqslant \pi
$$

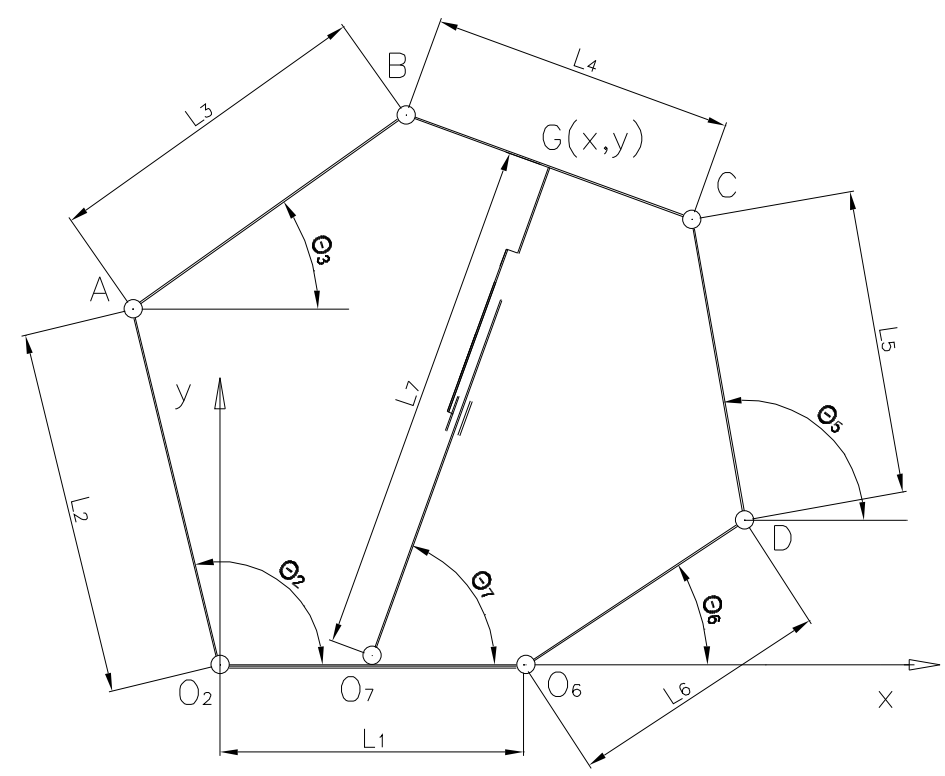

Figura 4.1: Diagrama do mecanismo

A segunda restrição se refere aos limites que a cadeia passiva $L_{7}$ poderá se estender ou contrair. Ao limite de extensão chamaremos de $L L$. Vamos então considerar os seguintes limites para $L_{7}$ :

$$
0 \leqslant L_{7} \leqslant L L
$$


As outras restrições se referem aos limites de extensão ou contração das pernas do mecanismo:

$$
\begin{aligned}
& \left|L_{2}-L_{3}\right| \leqslant\left\|B-O_{2}\right\| \leqslant L_{3}+L_{2}, \\
& \left|L_{6}-L_{5}\right| \leqslant\left\|C-O_{6}\right\| \leqslant L_{5}+L_{6} .
\end{aligned}
$$

As coordenadas dos vértices do mecanismo são:

$$
\begin{aligned}
& B\left(-\frac{L_{4}}{2} s_{7}+\frac{L_{1}}{2}+L_{7} c_{7}, \frac{L_{4}}{2} c_{7}+L_{7} s_{7}\right), \\
& O_{2}(0,0), \\
& C\left(\frac{L_{4}}{2} s_{7}+\frac{L_{1}}{2}+L_{7} c_{7},-\frac{L_{4}}{2} c_{7}+L_{7} s_{7}\right), \\
& O_{6}\left(L_{1}, 0\right) .
\end{aligned}
$$

Então,

$$
\begin{aligned}
\left\|B-O_{2}\right\| & =\sqrt{\left(-\frac{L_{4}}{2} s_{7}+\frac{L_{1}}{2}+L_{7} c_{7}\right)^{2}+\left(\frac{L_{4}}{2} c_{7}+L_{7} s_{7}\right)^{2}}, \\
\left\|C-O_{6}\right\| & =\sqrt{\left(\frac{L_{4}}{2} s_{7}+\frac{L_{1}}{2}+L_{7} c_{7}-L_{1}\right)^{2}+\left(-\frac{L_{4}}{2} c_{7}+L_{7} s_{7}\right)^{2}} .
\end{aligned}
$$

No anexo C apresentamos a listagem do programa Mathematica que constrói o gráfico da área de trabalho. Nesta listagem as coordenadas do ponto $G$ serão designadas por $(x, y)$.

A menor abscissa do ponto $G$ acontece quando $\theta_{7}=180^{\circ}$ e vale

$$
x_{\min }=\frac{L_{1}}{2}-L L
$$

A maior abscissa de $G$ acontece quando $\theta_{7}=0^{\circ}$ e vale

$$
x_{\max }=\frac{L_{1}}{2}+L L
$$

Por outro lado, a menor ordenada de $G$ é zero e a maior é $L L$.

Então podemos colocar

$$
\begin{aligned}
\frac{L_{1}}{2}-L L & \leqslant x \leqslant \frac{L_{1}}{2}+L L, \\
0 & \leqslant y \leqslant L L .
\end{aligned}
$$




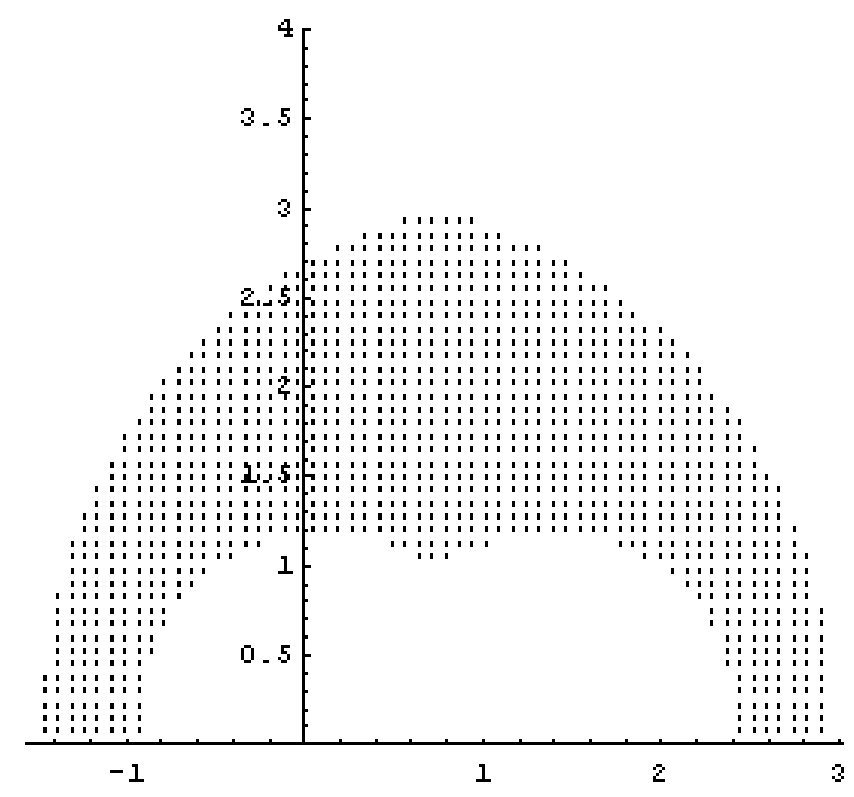

Figura 4.2: Espaço de trabalho método discretização

O comprimento da cadeia passiva é calculado por:

$$
L_{7}=\sqrt{\left(x-\frac{L_{1}}{2}\right)^{2}+y^{2}}
$$

Mas

$$
c_{7}=\frac{x-\frac{L_{1}}{2}}{L_{7}}
$$

e assim temos $c_{7}$ e $s_{7}$.

Se levarmos em conta as condições acima apresentadas podemos montar um vetor de coordenadas $\{x, y\}$ que as satisfaçam e construir um gráfico como o da fig.4.2 que foi feito utilizando-se o programa do Anexo C para os seguintes dados: $L_{1}=1,5, \quad L_{2}=L_{4}=L_{6}=1, \quad L_{3}=L_{5}=2$ e as distâncias entre os pontos do gráfico são iguais a $0,05 \cdot L_{1}$ tanto na direção $x$ como na direção $y$.

\subsection{Método geométrico}

\subsubsection{Curva externa da área de trabalho.}

A curva externa ocorre quando estendemos ao máximo as cadeias ativas do mecanismo, de forma que alcancem os comprimentos $L_{2}+L_{3}$ e $L_{5}+L_{6}$.

A fig. 4.3 mostra a construção da curva externa na qual consideramos um 
mecanismo tal que

$$
\begin{aligned}
L_{3} & =L_{5}, \\
L_{2} & =L_{6}, \\
L_{3} & >L_{2} \text { e } \quad L_{5}>L_{6} .
\end{aligned}
$$

O ponto 1 é a posição do ponto $G$ do mecanismo quando este está totalmente estendido de forma simétrica em relação à vertical que passa por $O_{7}$. O ponto 2 e demais pontos da curva externa direita ocorrem quando inclinamos a cadeia passiva para a direita mantendo-se estendidos e alinhados os elos da cadeia esquerda. Logicamente estamos supondo que a cadeia passiva tem curso suficiente para isto. A curva externa esquerda é o espelho da curva externa direita já que estamos considerando um mecanismo simétrico

\section{Determinação dos pontos da curva externa}

O ponto 2 é a posição do ponto $G$ do mecanismo quando inclinamos de um ângulo qualquer $\alpha$ a sua cadeia passiva, mantendo os elos $L_{2}$ e $L_{3}$ estendidos e alinhados. Para sua determinação devemos:

1) Com centro em $O_{2}$ e raio $L_{2}+L_{3}$ traçamos a circunferência $C_{1}$.

2) Por $O_{7}$ traçamos a semi-reta $r_{2}$ inclinada de $\alpha$ em relação à vertical $O_{7} 1$.

3) A uma distância $L_{4} / 2$ traçamos do lado esquerdo uma paralela à semi-reta $r_{2}$ e denominamos de $2 e$ o ponto de interseção desta paralela com a circunferência $C_{1}$.

4) Pelo ponto $2 e$ traçamos uma perpendicular à semi-reta $r_{2}$. A interseção destas nos dá o ponto 2 procurado.

Seguindo o mesmo raciocínio determinamos os demais pontos da curva externa. 


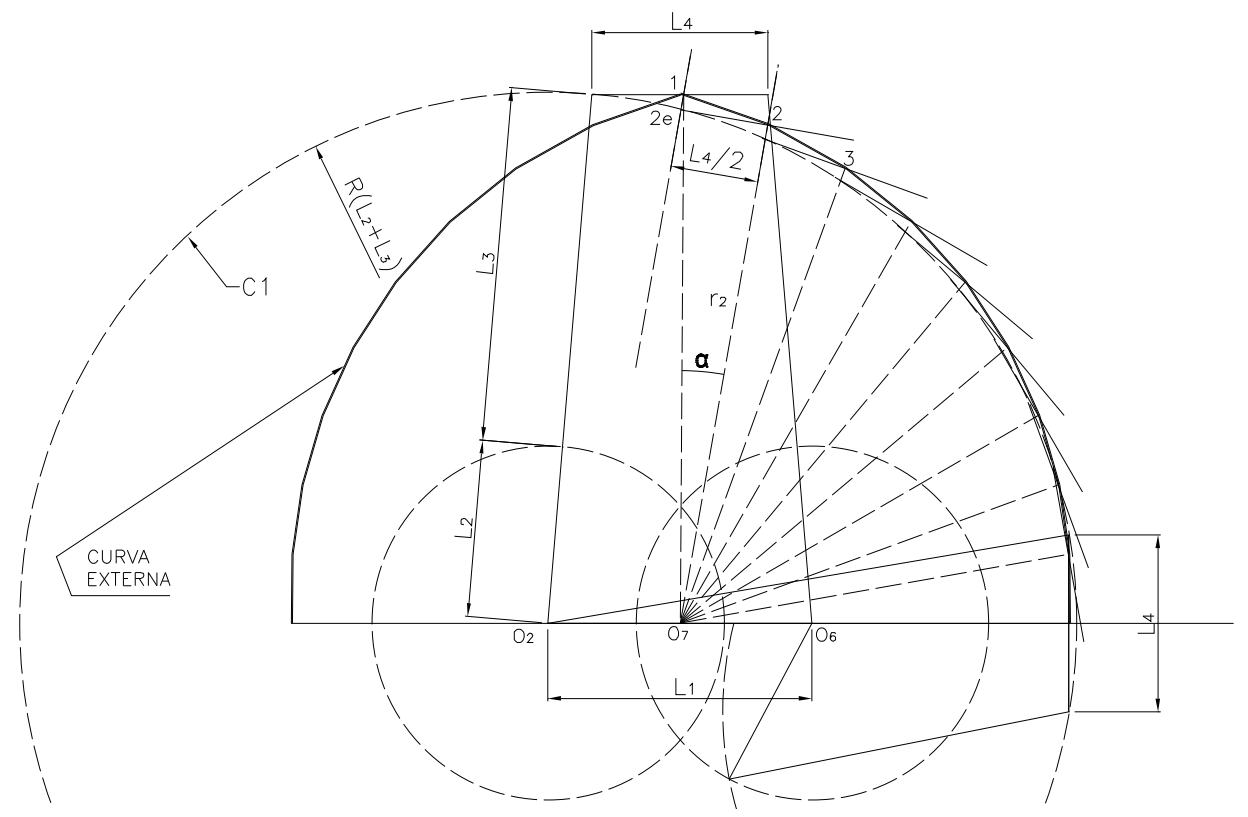

Figura 4.3: Curva externa do espaço de trabalho

\subsubsection{Curva interna da área de trabalho}

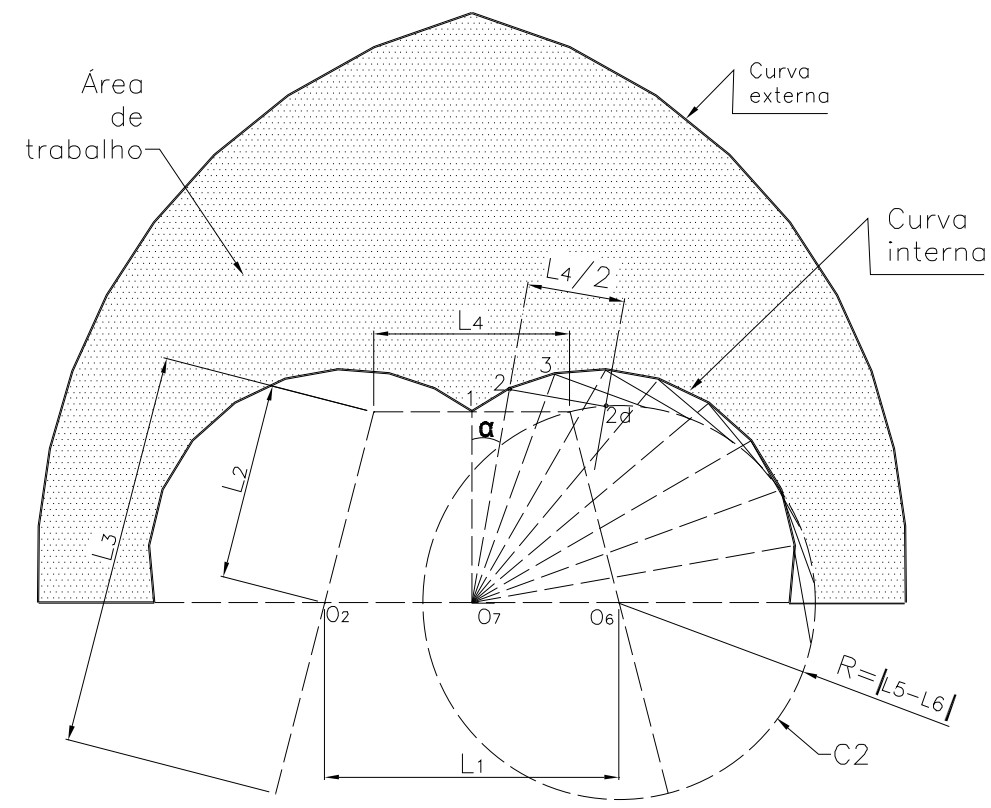

Figura 4.4: Curva interna e área de trabalho

A curva interna da área de trabalho dá o limite que o ponto $G$ pode atingir quando retraímos as cadeias ativas para $\left|L_{2}-L_{3}\right|$ e $\left|L_{5}-L_{6}\right|$.

A fig. 4.4 mostra como foi feita a construção da curva interna da área de trabalho. O espaço compreendido entre a curva externa e a curva inferna é a área 
de trabalho deste mecanismo.

O ponto 1 é a posição do ponto $G$ quando o mecanismo está totalmente contraído de forma simétrica em relação à vertical que passa por $O_{7}$. Quando inclinamos a cadeia passiva para direita obtemos o ponto 2 e demais pontos.

\section{Determinação dos pontos da curva interna}

1) Com centro em $O_{6}$ e raio $\left|L_{5}-L_{6}\right|$ traçamos a circunferência $C_{2}$.

2) Por $O_{7}$ traçamos a semi-reta $r_{2}$ com inclinação de $\alpha$ em relação à vertical $\mathrm{O}_{7} 1$.

3) A uma distância de $L_{4} / 2$ para direita traçamos uma paralela a $r_{2}$ e denominamos por $2 d$ o ponto de interseção desta paralela com a circunferência $C_{2}$.

4) Pelo ponto $2 d$ traçamos uma perpendicular à semi-reta $r_{2}$ e na interseção com esta temos o ponto 2.

Os demais pontos da curva são determinados de forma análoga.

\subsection{Otimização do espaço de trabalho}

Interessa-nos agora determinar os comprimentos dos elos das cadeias ativas de forma a otimizar o espaço de trabalho do mecanismo em relação ao espaço de trabalho específico da cadeia passiva.

Vamos impor inicialmente as seguintes condições:

- Mecanismo simétrico em relação à cadeia passiva, isto é, $L_{2}=L_{6}$ e $L_{3}=L_{5}$. Deste modo podemos fazer o estudo só para um lado do mecanismo.

- São fornecidos os comprimentos da base $\left(L_{1}\right)$, da plataforma móvel $\left(L_{4}\right)$, da cadeia passiva totalmente estendida $\left(L_{7 E}\right)$, totalmente contraída $\left(L_{7 C}\right)$ bem como os limites de inclinação da cadeia passiva, isto, é $0 \leqslant \theta_{7} \gtrless \pi$.

Conforme podemos ver pela fig.4.5 o espaço de trabalho alcançado pela cadeia passiva isoladamente é a área da semi-coroa $G_{1} G_{2} G_{3} G_{4} G_{5} G_{6}$ cujo raio externo é $L_{7 E}$ e cujo raio interno é $L_{7 C}$. 


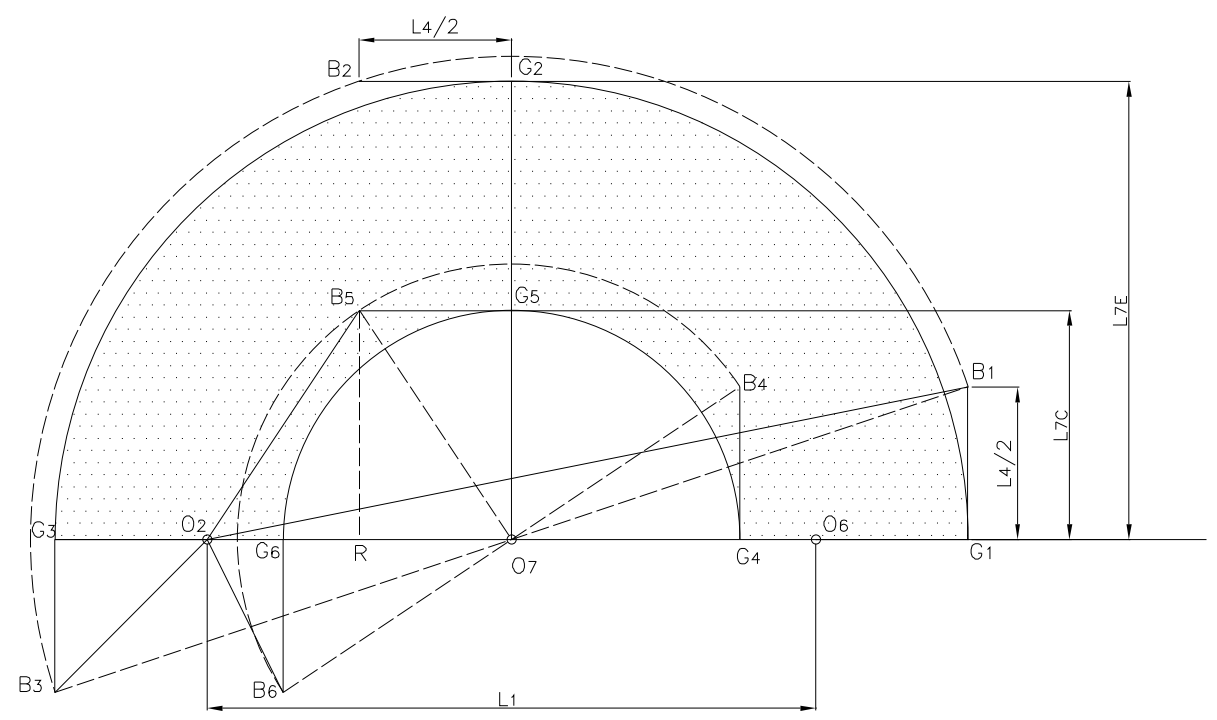

Figura 4.5: Otimização do espaço de trabalho

Quando a cadeia passiva está totalmente estendida a garra descreve o arco $G_{1} G_{2} G_{3}$ e o ponto $B$ (extremidade esquerda da plataforma móvel) descreve o arco $B_{1} B_{2} B_{3}$ de raio $O_{7} B_{1}$.

A maior distância entre o ponto $B$ e a junta $O_{2}$ ocorre quando $B$ está em $B_{1}$. Assim sendo podemos escrever:

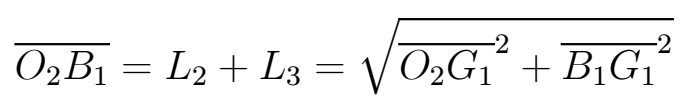

mas

$$
\begin{gathered}
\overline{O_{2} G_{1}}=L_{7 E}+\frac{L_{1}}{2} \\
\overline{B_{1} G_{1}}=\frac{L_{4}}{2}
\end{gathered}
$$

donde

$$
L_{2}+L_{3}=\sqrt{\left(L_{7 E}+\frac{L_{1}}{2}\right)^{2}+\left(\frac{L_{4}}{2}\right)^{2}}
$$

A menor distância entre o ponto $B$ e a junta $O_{2}$ ocorre quando $B$ está em $B_{3}$, isto é,

$$
\overline{O_{2} B_{3}}=\sqrt{{\overline{O_{2} G_{3}}}^{2}+{\overline{B_{3} G_{3}}}^{2}}
$$

mas

$$
\begin{gathered}
\overline{O_{2} G_{3}}=L_{7 E}-\frac{L_{1}}{2} \\
\overline{B_{3} G_{3}}=\frac{L_{4}}{2}
\end{gathered}
$$


donde

$$
\overline{O_{2} B_{3}}=\sqrt{\left(L_{7 E}-\frac{L_{1}}{2}\right)^{2}+\left(\frac{L_{4}}{2}\right)^{2}}
$$

Quando a cadeia passiva está totalmente contraída a garra descreve o arco $G_{4} G_{5} G_{6}$ e o ponto B descreve o arco $B_{4} B_{5} B_{6}$ de raio $O_{7} B_{4}$.

A menor distância entre o ponto $B$ e a junta $O_{2}$ ocorre quando $B$ está em $B_{5}$ ou em $B_{6}$. Então temos:

$$
\overline{O_{2} B_{5}}=\sqrt{{\overline{O_{2} R}}^{2}+{\overline{B_{5} R}}^{2}}
$$

mas

$$
\begin{gathered}
\overline{O_{2} R}=\frac{L_{1}}{2}-\frac{L_{4}}{2} \\
\overline{B_{5} R}=L_{7 C}
\end{gathered}
$$

donde

$$
\overline{O_{2} B_{5}}=\sqrt{\left(\frac{L_{1}}{2}-\frac{L_{4}}{2}\right)^{2}+L_{7 C}^{2}}
$$

e

$$
\overline{O_{2} B_{6}}=\sqrt{{\overline{O_{2} G_{6}}}^{2}+{\overline{B_{6} G_{6}}}^{2}}
$$

mas

donde

$$
\begin{gathered}
\overline{O_{2} G_{6}}=\frac{L_{1}}{2}-L_{7 C} \\
\overline{B_{6} G_{6}}=\frac{L_{4}}{2}
\end{gathered}
$$

$$
\overline{O_{2} B_{6}}=\sqrt{\left(\frac{L_{1}}{2}-L_{7 C}\right)^{2}+\left(\frac{L_{4}}{2}\right)^{2}}
$$

Então $\left|L_{2}-L_{3}\right|$ será a menor das distâncias $\overline{O_{2} B_{3}}, \overline{O_{2} B_{5}}$ e $\overline{O_{2} B_{6}}$.

Conclusão: Para otimizarmos o espaço de trabalho devemos determinar os comprimentos dos elos tal que a soma dos elos $L_{2}+L_{3}$ seja determinada conforme a equação 4.1 e o módulo de sua diferença $\left|L_{2}-L_{3}\right|$ seja igual ao menor dos valores encontrados pelas equações $4.2,4.3$ e 4.4 . 


\section{Capítulo 5: TRAJETÓRIAS DA GARRA}

\subsection{Introdução}

Na robótica busca-se sempre melhorar a eficiência das operações tanto no que se refere à precisão dos movimentos como também à sua rapidez. Mas, acima de um determinado nível de velocidade, tanto a partida como a parada de um mecanismo não pode ser feita de forma brusca, pois isto fatalmente levará a efeitos indesejáveis como solavancos, vibrações, golpes bruscos ou até ao próprio colapso do mecanismo. Uma forma de minimizar estes efeitos indesejáveis é fazer com que o mecanismo inicie o movimento, acelerando uniformemente, até atingir sua velocidade máxima. Da mesma forma quando tiver que parar em determinado ponto o mecanismo deverá previamente reduzir sua velocidade de maneira gradativa até sua parada total. As rampas de aceleração e desaceleração são justamente estes movimentos uniformemente variados que impomos ao mecanismo nos extremos de seu deslocamento para suavizar sua operação.

É importante ressaltar que o movimento que tratamos aqui se refere sempre ao centro de gravidade da plataforma móvel, o qual definimos como ponto de referência da garra do mecanismo, como já dissemos anteriormente.

A aceleração, a desaceleração e a velocidade máxima são variáveis de projeto.

Um problema que devemos considerar no estudo da trajetória realizada pelo mecanismo é que a mesma deve estar contida no espaço de trabalho e que não haja singularidades que possam afetar esta trajetória. Conforme Koren o planejamento da trajetória significa a determinação da trajetória real, ou caminho ("path") ao longo do qual o órgão terminal do robô se moverá[17]. Com base neste livro de Koren desenvolvemos nosso trabalho sobre as rampas de aceleração e desaceleração, porem consideramos sempre uma trajetória regular e contínua dentro do espaço de trabalho, isto é, sem variações bruscas de trajeto impostas por descontinuidades do espaço de trabalho ou por qualquer tipo de singularidade.

\subsection{Deslocamentos retilíneos}

O deslocamento deve ser realizado conforme mostrado na fig. 5.1 onde do instante $t_{A}$ a $t_{B}$ temos um movimento uniformemente acelerado, de $t_{B}$ a $t_{C}$ ocorre a velocidade máxima (constante) e de $t_{C}$ a $t_{D}$ temos o movimento uniformemente retardado. Considerando que a aceleração e a desaceleração têm o mesmo módulo 
$a$, e que no instante $t_{A}$ a velocidade é nula (o mecanismo parte do repouso), podemos escalarmente escrever:

$$
\begin{gathered}
t_{A} \leqslant t<t_{B}, \quad v=a\left(t-t_{A}\right) \\
t_{B} \leqslant t \leqslant t_{C}, \quad v=v_{\max }, \quad a=0 \\
t_{C} \leqslant t<t_{D}, \quad v=v_{\max }-a\left(t-t_{C}\right)
\end{gathered}
$$

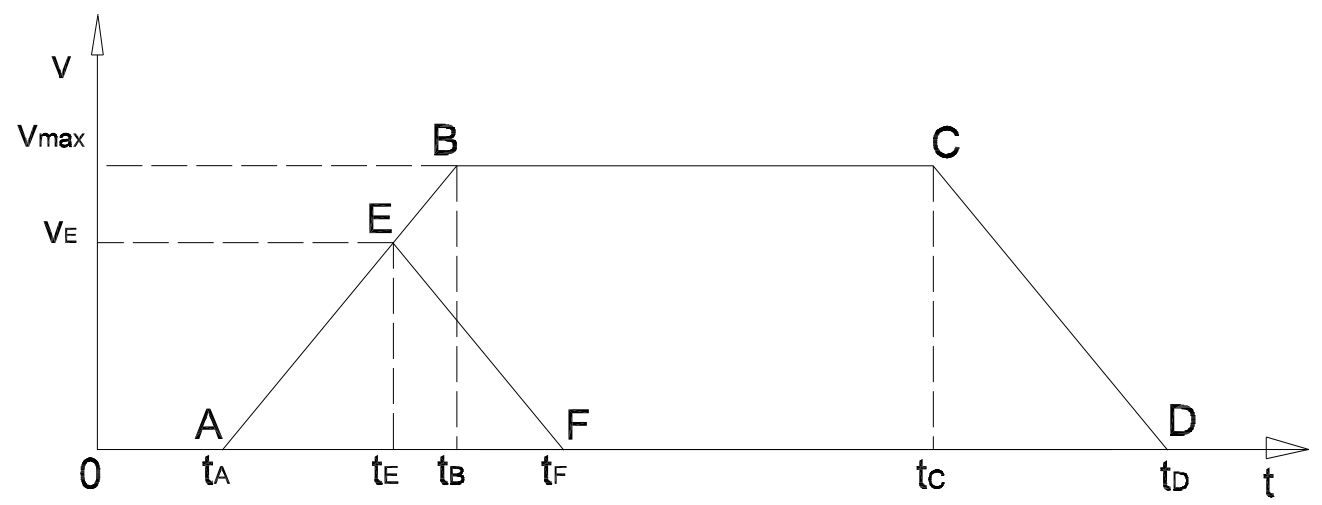

Figura 5.1: Trajetória retilínea: curva tempo x velocidade.

O espaço percorrido é dado pela área da curva com o eixo horizontal.

Então o espaço percorrido no tempo $t$ entre $t_{A}$ e $t_{B}$ é:

$$
s=\frac{v\left(t-t_{A}\right)}{2}=\frac{a\left(t-t_{A}\right)^{2}}{2}
$$

Portanto no instante $t_{B}$ o espaço percorrido é dado por:

$$
s_{A B}=\frac{v_{\max }\left(t_{B}-t_{A}\right)}{2}=\frac{a\left(t_{B}-t_{A}\right)^{2}}{2}
$$

No trecho BC a velocidade é constante e, portanto, a aceleração é nula. Então para um tempo $t$ entre os instantes $t_{B}$ e $t_{C}$ o espaço percorrido no regime de velocidade constante será de:

$$
s=v_{\max }\left(t-t_{B}\right)
$$


Logo o espaço total percorrido durante todo o regime de velocidade constante é:

$$
s_{B C}=v_{\max }\left(t_{C}-t_{B}\right)
$$

E o espaço total percorrido durante o regime de desaceleração é:

$$
s_{C D}=\frac{v_{\max }\left(t_{D}-t_{C}\right)}{2}
$$

Então o espaço total percorrido é:

$$
s_{t}=s_{A B}+s_{B C}+s_{C D}=\frac{v_{\max }\left(t_{B}-t_{A}\right)}{2}+v_{\max }\left(t_{C}-t_{B}\right)+\frac{v_{\max }\left(t_{D}-t_{C}\right)}{2}
$$

isto é,

$$
s_{t}=\frac{v_{\max }\left(t_{D}+t_{C}-t_{B}-t_{A}\right)}{2}
$$

Existem casos em que o espaço a ser percorrido é tão pequeno que o mecanismo nem chega a atingir a velocidade máxima e já terá que desacelerar para parar. É o caso mostrado na fig. 5.1 quando o mecanismo parte de A e acelera até o ponto E e desacelera até parar em F.

A velocidade no instante $t_{E}$ tem o valor de

$$
v_{E}=a\left(t_{E}-t_{A}\right)
$$

e como estamos impondo que o módulo da aceleração é igual ao da desaceleração, o espaço total percorrido pelo mecanismo é calculado por:

$$
s_{A F}=a\left(t_{E}-t_{A}\right)^{2}
$$

Como nos interessa trabalhar mais com o espaço percorrido do que com o tempo, a partir de agora vamos lançar mão da fórmula de Torricelli para desenvolver nossos cálculos. A fig. 5.2 mostra a curva da velocidade em função do espaço para as três fases do movimento: aceleração, velocidade máxima e desaceleração. 


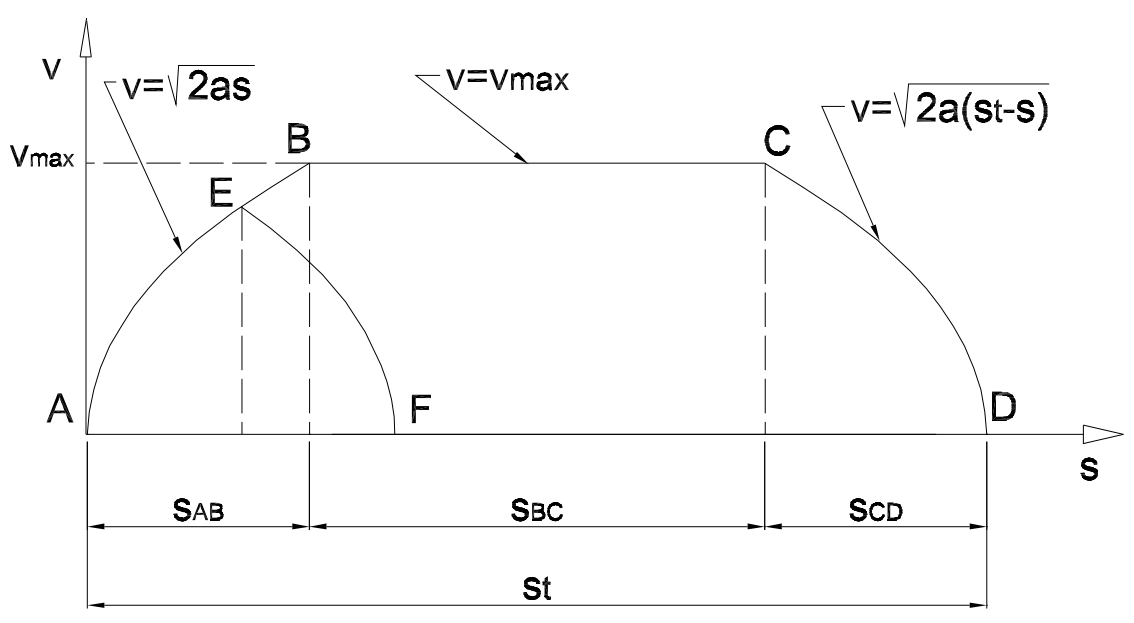

Figura 5.2: Trajetória retilínea: curva espaço x velocidade.

Vamos dividir nosso problema em duas condições: a primeira condição é quando o mecanismo atinge a velocidade máxima, isto é, $s_{B C}>0$ e a segunda condição é quando o mecanismo não chega a atingir a velocidade máxima. Para cada uma destas condições analisaremos as particularidades de cada fase.

\subsubsection{Primeira condição: $s_{B C} \geqslant 0$}

Trecho AB: $0<s<s_{A B}$

A velocidade é calculada por

$$
v=\sqrt{2 a s}
$$

Trecho BC: $s_{A B}<s<s_{A B}+s_{B C}$

A aceleração é nula e a velocidade é constante e igual a

$$
v=v_{\max }
$$

Trecho CD: $s_{A B}+s_{B C}<s<s_{t}$

A velocidade é calculada por

$$
v=\sqrt{2 a\left(s_{t}-s\right)}
$$




\subsubsection{Segunda condição: $s_{B C}<0$}

Trecho AE: $s<s_{t} / 2$

A velocidade é calculada por

$$
v=\sqrt{2 a s}
$$

Trecho EF: $s>s_{t} / 2$

A velocidade é calculada por

$$
v=\sqrt{2 a\left(s_{t}-s\right)}
$$

\subsection{Deslocamento retilíneo numa direção qual- quer}

Vamos supor agora que o deslocamento AD do mecanismo seja numa direção que forma um ângulo $\varphi$ com a horizontal. Nosso objetivo é obter as componentes escalares das velocidades e acelerações em relação aos eixos de coordenas, isto é $v_{x}, v_{y}, a_{x}$ e $a_{y}$ as quais serão utilizadas no cálculo dos torques e forças de reação (Análise Dinâmica).

\subsubsection{Primeira condição: $s_{B C} \geqslant 0$}

Trecho AB: $0<s<s_{A B}$

As componentes da velocidade são:

$$
\begin{aligned}
& v_{x}=\sqrt{2 a s} c \varphi \\
& v_{y}=\sqrt{2 a s} s \varphi
\end{aligned}
$$

As componentes da aceleração são:

$$
\begin{aligned}
& a_{x}=a c \varphi \\
& a_{y}=a s \varphi
\end{aligned}
$$


Trecho BC: $s_{A B}<s<s_{A B}+s_{B C}$

As componentes da velocidade são:

$$
\begin{aligned}
& v_{x}=v_{\max } c \varphi \\
& v_{y}=v_{\max } s \varphi
\end{aligned}
$$

Não há componentes da aceleração.

Trecho CD: $s_{A B}+s_{B C}<s<s_{t}$

As componentes da velocidade são:

$$
\begin{aligned}
& v_{x}=\sqrt{2 a\left(s_{t}-s\right)} c \varphi \\
& v_{y}=\sqrt{2 a\left(s_{t}-s\right)} s \varphi
\end{aligned}
$$

As componentes da aceleração são:

$$
\begin{aligned}
& a_{x}=-a c \varphi \\
& a_{y}=-a s \varphi
\end{aligned}
$$

\subsubsection{Segunda condição: $s_{B C}<0$}

Trecho AE: $s<s_{t} / 2$

As componentes da velocidade são:

$$
\begin{aligned}
& v_{x}=\sqrt{2 a s} c \varphi \\
& v_{y}=\sqrt{2 a s} s \varphi
\end{aligned}
$$

As componentes da aceleração são:

$$
\begin{aligned}
& a_{x}=a c \varphi \\
& a_{y}=a s \varphi
\end{aligned}
$$


Trecho EF: $s>s_{t} / 2$

As componentes da velocidade são:

$$
\begin{aligned}
& v_{x}=\sqrt{2 a\left(s_{t}-s\right)} c \varphi \\
& v_{y}=\sqrt{2 a\left(s_{t}-s\right)} s \varphi
\end{aligned}
$$

As componentes da aceleração são:

$$
\begin{aligned}
& a_{x}=-a c \varphi \\
& a_{y}=-a s \varphi
\end{aligned}
$$

Consequentemente quando acionamos o mecanismo para percorrer uma trajetória reta, dentro da sua área de trabalho, podemos, utilizando as equações acima, calcular as componentes da velocidade e aceleração, as quais serão necessárias para o cálculo dos torques dos atuadores em qualquer ponto percurso.

No AnexoD mostramos a listagem de programa do software Mathematica que calcula $v_{x}, v_{y}, a_{x}$ e $a_{y}$ para qualquer ponto situado na trajetória.

Neste programa consideramos os dados de projeto:

-Velocidade máxima: $v_{\max }=1,0 \mathrm{~m} / \mathrm{s}$

-Módulo da aceleração igual da desaceleração: $a=10,0 \mathrm{~m} / \mathrm{s}^{2}$

Consequentemente o espaço percorrido na aceleração $\left(s_{A B}\right)$ e desaceleração $\left(s_{C D}\right)$ tem o mesmo comprimento, isto é:

$$
s_{A B}=s_{C D}=\frac{v_{\max }^{2}}{2 a_{t}}=0,05 \mathrm{~m}
$$

então

$$
S_{B C}=s_{t}-0,1
$$

Devemos entrar com os seguintes dados:

-Deslocamento total: $s_{t}$

-Inclinação deste deslocamento em relação ao eixo $x: \varphi$

-Deslocamento do ponto onde queremos achar os resultados: $s$.

O programa mostra o resultado na forma de um vetor: $\left\{v_{x}, v_{y}, a_{x}, a_{y}\right\}$. 


\subsection{Deslocamento circular}

Vamos considerar que a garra do mecanismo parte do ponto A e sofre um deslocamento circular de raio $r$ até parar no ponto D, conforme mostra a fig.5.3. Portanto a trajetória é um arco AD e, no caso, o seu sentido é anti-horário e o seu centro está na origem dos eixos de coordenadas. No trecho da rampa de aceleração que vai de $\mathrm{A}$ até $\mathrm{B}$ temos uma aceleração tangencial constante que denominaremos de $\overrightarrow{a_{t}}$. No trecho BC a velocidade tangencial é constante, portanto $\overrightarrow{a_{t}}=0$. No trecho da rampa de desaceleração CD temos uma desaceleração cujo módulo é o mesmo da aceleração.

Conforme vimos no caso da trajetória reta podemos ter situações nas quais o percurso é tão pequeno que o mecanismo nem chega a atingir a velocidade máxima e já tem que desacelerar para parar. Vamos então considerar em primeiro lugar a condição de que o mecanismo atinge a velocidade máxima e depois vamos considerar a condição na qual o mecanismo não chega a atingir esta velocidade.

Como agora lidaremos com ângulos, vamos estabelecer que todos os ângulos serão medidos no sentido anti-horário à partir do eixo horizontal de coordenadas.

Inicialmente desenvolveremos a teoria considerando o centro da trajetória coincidindo com a origem dos eixos de coordenadas, como mostrado na fig5.3 e depois o desenvolvimento será feito para o caso mais geral, em que o centro da trajetória não coincide com a origem dos eixos de coordenadas.

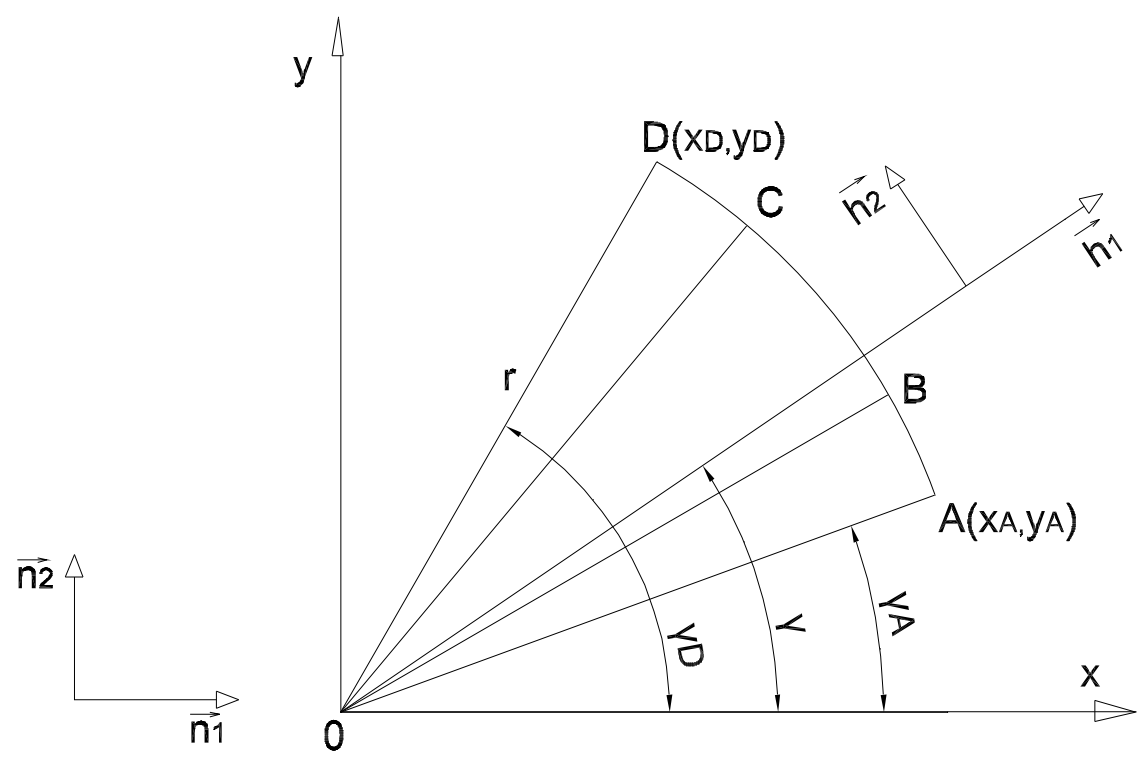

Figura 5.3: Trajetória circular com centro do arco na origem dos eixos de coordenadas. 
É importante observar que no caso geral (fig.5.4), em sendo dados o ponto de início de movimento (A) e o ponto do final do movimento (D), podemos ter quatro arcos de mesmo raio que vai de $\mathrm{A}$ até $\mathrm{D}$, isto é:

-Arco $\mathrm{AD}$ com centro à esquerda da corda $\mathrm{AD}$ e sentido horário.

-Arco $\mathrm{AD}$ com centro à esquerda da corda $\mathrm{AD}$ e sentido anti- horário.

-Arco $\mathrm{AD}$ com centro à direita da corda $\mathrm{AD}$ e sentido horário.

-Arco $\mathrm{AD}$ com centro à direita da corda $\mathrm{AD}$ e sentido anti-horário.

Do mesmo modo que no caso do deslocamento retilínio, consideraremos nos exemplos de aplicação os seguintes dados de projeto:

-Velocidade tangencial máxima: $v_{\max }=1 \mathrm{~m} / \mathrm{s}$

-Aceleração tangencial: $a_{t}=10 \mathrm{~m} / \mathrm{s}^{2}$

-Módulo da aceleração tangencial igual ao da desaceleração.

Consequentemente o trecho de aceleração e desaceleração têm o mesmo comprimento, isto é,

$$
s_{A B}=s_{C D}=\frac{v_{\max }^{2}}{2 a_{t}}=0,05 m
$$

Onde $s_{A B}$ e $s_{C D}$ são os comprimentos dos arcos que vão de $\mathrm{A}$ até $\mathrm{B}$ e de $\mathrm{C}$ até $\mathrm{D}$ respectivamente.

\subsection{Centro do arco na origem dos eixos de co- ordenadas}

\subsubsection{Primeira condição: $s_{B C} \geqslant 0$}

Nesta condição o mecanismo chega a atingir a velocidade máxima e com esta velocidade percorre o trecho que vai de B até C, cujo comprimento estamos chamando de $s_{B C}$.

O espaço total a ser percorrido pelo mecanismo do ponto de partida até a parada é dado por:

$$
s_{t}=r\left(\gamma_{D}-\gamma_{A}\right)
$$

O espaço a ser percorrido no trecho onde a velocidade é constante é dado por:

$$
s_{B C}=s_{t}-s_{A B}-s_{C D}=r\left(\gamma_{D}-\gamma_{A}\right)-s_{A B}-s_{C D}
$$

O espaço a ser percorrido até um ponto qualquer da trajetória a partir do ponto inicial A é dado por:

$$
s=r\left(\gamma-\gamma_{A}\right)
$$


Temos três casos a considerar nesta primeira condição:

1 - O ponto onde queremos determinar a velocidade e aceleração está situado no trecho de aceleração: $\mathrm{AB}$.

2 - O ponto está no trecho de velocidade constante: BC.

3 - O ponto está no trecho de desaceleração: CD.

Trecho AB: $0 \leqslant s \leqslant s_{A B}$

A velocidade tangencial em um ponto qualquer neste trecho é dada por:

$$
\vec{v}=v \overrightarrow{h_{2}}
$$

onde

$$
v=\sqrt{2 a_{t} s}
$$

então

$$
\vec{v}=\sqrt{2 a_{t} s} \overrightarrow{h_{2}}
$$

A aceleração tangencial é

$$
\overrightarrow{a_{t}}=a_{t} \overrightarrow{h_{2}}
$$

A aceleração centrípeta é

$$
\overrightarrow{a_{c}}=-a_{c} \overrightarrow{h_{1}}
$$

onde

$$
a_{c}=\frac{v^{2}}{r}=\frac{2 a_{t} s}{r}
$$

então

$$
\overrightarrow{a_{c}}=-\frac{2 a_{t} s}{r} \overrightarrow{h_{1}}=-2 a_{t}\left(\gamma-\gamma_{A}\right) \overrightarrow{h_{1}}
$$

As componentes da velocidade tangencial são:

$$
\overrightarrow{v_{x}}=v\left(\overrightarrow{h_{2}} \cdot \overrightarrow{n_{1}}\right) \overrightarrow{n_{1}}=-v s \gamma \overrightarrow{n_{1}}
$$

ou seja,

$$
\overrightarrow{v_{x}}=-\sqrt{2 a_{t} s} s \gamma \overrightarrow{n_{1}}
$$

e

$$
\overrightarrow{v_{y}}=v\left(\overrightarrow{h_{2}} \cdot \overrightarrow{n_{2}}\right) \overrightarrow{n_{2}}=v c \gamma \overrightarrow{n_{2}}
$$

ou seja,

$$
\overrightarrow{v_{y}}=\sqrt{2 a_{t} s} c \gamma \overrightarrow{n_{2}}
$$


As componentes da aceleração tangencial são:

$$
\begin{aligned}
& \overrightarrow{a_{t x}}=a_{t}\left(\overrightarrow{h_{2}} \cdot \overrightarrow{n_{1}}\right) \overrightarrow{n_{1}}=-a_{t} s \gamma \overrightarrow{n_{1}} \\
& \overrightarrow{a_{t y}}=a_{t}\left(\overrightarrow{h_{2}} \cdot \overrightarrow{n_{2}}\right) \overrightarrow{n_{2}}=a_{t} c \gamma \overrightarrow{n_{2}}
\end{aligned}
$$

As componentes da aceleração centrípeta são:

$$
\overrightarrow{a_{c x}}=-a_{c}\left(\overrightarrow{h_{1}} \cdot \overrightarrow{n_{1}}\right) \overrightarrow{n_{1}}=-a_{c} c \gamma \overrightarrow{n_{1}}
$$

ou seja,

$$
\overrightarrow{a_{c x}}=-\frac{2 a_{t} s}{r} c \gamma \overrightarrow{n_{1}}=-2 a_{t}\left(\gamma-\gamma_{A}\right) c \gamma \overrightarrow{n_{1}}
$$

e

$$
\overrightarrow{a_{c y}}=-a_{c}\left(\overrightarrow{h_{1}} \cdot \overrightarrow{n_{2}}\right) \overrightarrow{n_{2}}=-a_{c} s \gamma \overrightarrow{n_{2}}
$$

ou seja,

$$
\overrightarrow{a_{c y}}=-\frac{2 a_{t} s}{r} s \gamma \overrightarrow{n_{2}}=-2 a_{t}\left(\gamma-\gamma_{A}\right) s \gamma \overrightarrow{n_{2}}
$$

Consequentemente as componentes finais das acelerações são:

$$
\overrightarrow{a_{x}}=\overrightarrow{a_{t x}}+\overrightarrow{a_{c x}}
$$

ou seja,

$$
\overrightarrow{a_{x}}=\left[-a_{t} s \gamma-2 a_{t}\left(\gamma-\gamma_{A}\right) c \gamma\right] \overrightarrow{n_{1}}
$$

$\mathrm{e}$

$$
\overrightarrow{a_{y}}=\overrightarrow{a_{t y}}+\overrightarrow{a_{c y}}
$$

ou seja,

$$
\overrightarrow{a_{y}}=\left[a_{t} c \gamma-2 a_{t}\left(\gamma-\gamma_{A}\right) s \gamma\right] \overrightarrow{n_{2}}
$$

Trecho BC: $s_{A B} \leqslant s \leqslant s_{A B}+s_{B C}$

Neste trecho a velocidade tangencial é constante e máxima. Não há aceleração tangencial, mas somente a aceleração centrípeta. Temos então:

$$
\begin{gathered}
\vec{v}=v_{\max } \overrightarrow{h_{2}} \\
\overrightarrow{a_{t}}=0 \\
\overrightarrow{a_{c}}=-\frac{v_{\max }^{2}}{r} \overrightarrow{h_{1}}
\end{gathered}
$$


Componentes da velocidade tangencial

$$
\begin{aligned}
& \overrightarrow{v_{x}}=v_{\max }\left(\overrightarrow{h_{2}} \cdot \overrightarrow{n_{1}}\right) \overrightarrow{n_{1}}=-v_{\max } s \gamma \overrightarrow{n_{1}} \\
& \overrightarrow{v_{y}}=v_{\max }\left(\overrightarrow{h_{2}} \cdot \overrightarrow{n_{2}}\right) \overrightarrow{n_{2}}=v_{\max } c \gamma \overrightarrow{n_{2}}
\end{aligned}
$$

As componentes da aceleração são constituídas somente pelas componentes da aceleração centrípeta já que não existe aceleração tangencial. Então

$$
\begin{aligned}
& \overrightarrow{a_{x}}=-\frac{v_{\max }^{2}}{r} c \gamma \overrightarrow{n_{1}} \\
& \overrightarrow{a_{y}}=-\frac{v_{\max }^{2}}{r} s \gamma \overrightarrow{n_{2}}
\end{aligned}
$$

Trecho CD: $s_{A B}+s_{B C} \leqslant s \leqslant s_{t}$

Com raciocínio análogo ao que fizemos para o trecho $\mathrm{AB}$ temos:

Velocidade tangencial:

$$
\vec{v}=\sqrt{2 a_{t} s} \overrightarrow{h_{2}}
$$

Componentes da velocidade tangencial

$$
\begin{aligned}
& \overrightarrow{v_{x}}=-\sqrt{2 a_{t} r\left[\gamma_{D}-\gamma\right]} s \gamma \overrightarrow{n_{1}} \\
& \overrightarrow{v_{y}}=\sqrt{2 a_{t} r\left[\gamma_{D}-\gamma\right]} c \gamma \overrightarrow{n_{2}}
\end{aligned}
$$

Aceleração tangencial:

$$
\overrightarrow{a_{t}}=a_{t} \overrightarrow{h_{2}}
$$

Aceleração centrípeta

$$
\overrightarrow{a_{c}}=-2 a_{t}\left(\gamma_{D}-\gamma\right) \overrightarrow{h_{1}}
$$

Componentes das acelerações

$$
\begin{aligned}
\overrightarrow{a_{x}} & =\left[\begin{array}{ll}
a_{t} s \gamma-2 a_{t}\left(\gamma_{D}-\gamma\right) c \gamma
\end{array}\right] \overrightarrow{n_{1}} \\
\overrightarrow{a_{y}} & =\left[-a_{t} c \gamma-2 a_{t}\left(\gamma_{D}-\gamma\right) s \gamma\right] \overrightarrow{n_{2}}
\end{aligned}
$$

\subsubsection{Segunda condição: $s_{B C}<0$}

Nesta condição o mecanismo antes de atingir a velocidade máxima começa a desacelerar até parar. Temos então somente duas fases: fase de aceleração e fase 
de desaceleração.

Fase aceleração: $s<s_{t} / 2$

São as mesmas equações do trecho $\mathrm{AB}$ da condição anterior:

Velocidade tangencial:

$$
\vec{v}=\sqrt{2 a_{t} s} \overrightarrow{h_{2}}
$$

Componentes da velocidade tangencial

$$
\begin{aligned}
& \overrightarrow{v_{x}}=-\sqrt{2 a_{t} s} s \gamma \overrightarrow{n_{1}} \\
& \overrightarrow{v_{y}}=\sqrt{2 a_{t} s} c \gamma \overrightarrow{n_{2}}
\end{aligned}
$$

Aceleração tangencial

$$
\overrightarrow{a_{t}}=a_{t} \overrightarrow{h_{2}}
$$

Aceleração centrípeta

$$
\overrightarrow{a_{c}}=-2 a_{t}\left(\gamma-\gamma_{A}\right) \overrightarrow{h_{1}}
$$

Componentes das acelerações

$$
\begin{aligned}
& \overrightarrow{a_{x}}=\left[-a_{t} s \gamma-2 a_{t}\left(\gamma-\gamma_{A}\right) c \gamma\right] \overrightarrow{n_{1}} \\
& \overrightarrow{a_{y}}=\left[a_{t} c \gamma-2 a_{t}\left(\gamma-\gamma_{A}\right) s \gamma\right] \overrightarrow{n_{2}}
\end{aligned}
$$

Fase desaceleração: $s>s_{t} / 2$

São as mesmas equações do trecho CD da condição anterior.

Velocidade tangencial e componentes:

$$
\begin{gathered}
\vec{v}=\sqrt{2 a_{t} s} \overrightarrow{h_{2}} \\
\overrightarrow{v_{x}}=-\sqrt{2 a_{t} r\left[\gamma_{D}-\gamma\right]} s \gamma \overrightarrow{n_{1}} \\
\overrightarrow{v_{y}}=\sqrt{2 a_{t} r\left[\gamma_{D}-\gamma\right]} c \gamma \overrightarrow{n_{2}}
\end{gathered}
$$

Aceleração tangencial

$$
\overrightarrow{a_{t}}=a_{t} \overrightarrow{h_{2}}
$$


Aceleração centrípeta

$$
\overrightarrow{a_{c}}=-2 a_{t}\left(\gamma_{D}-\gamma\right) \overrightarrow{h_{1}}
$$

Componentes das acelerações

$$
\begin{aligned}
& \overrightarrow{a_{x}}=\left[a_{t} s \gamma-2 a_{t}\left(\gamma_{D}-\gamma\right) c \gamma\right] \overrightarrow{n_{1}} \\
& \overrightarrow{a_{y}}=\left[-a_{t} c \gamma-2 a_{t}\left(\gamma_{D}-\gamma\right) s \gamma\right] \overrightarrow{n_{2}}
\end{aligned}
$$

O anexoE é utilizado para o caso em que o centro do arco coincide com a origem dos eixos de coordenadas principal. Nele devemos entrar com o raio do arco , a aceleração tangencial (módulo), o ângulo do raio que corresponde ao ponto $\mathrm{P}$ (ponto no qual queremos achar as componentes da velocidade e aceleração), o ângulo do raio que corresponde ao ponto inicial do movimento (ponto A), o ângulo do raio que corresponde ao ponto final do movimento (ponto D) e com o sentido (sinal) do arco (horário ou anti-horário).

O resultado será um vetor com as componentes da velocidade e aceleração para o ponto $\mathrm{P}$

\subsection{Centro do arco fora da origem dos eixos de coordenadas. Caso geral.}

No caso geral temos duas circunferências de mesmo raio $r$ que passam por A e $\mathrm{D}$ cujos centos são $O_{1}$ e $O_{2}$. Para irmos de A até $\mathrm{D}$ existem 4 arcos trajetórias conforme ilustrados na fig. 5.4. Enquanto que no caso anterior eram dados os ângulos $\gamma_{A}$ e $\gamma_{D}$, e definíamos apenas o sentido do arco, agora são dados o ponto inicial do movimento $A\left(x_{A}, y_{A}\right)$ e o ponto final $D\left(x_{D}, y_{D}\right)$ e devemos definir não só o sentido do arco, como também o seu centro. Para usar as mesmas fórmulas anteriores devemos agora determinar as coordenadas dos dois centros dos arcos, os ângulos $\gamma_{A}$ e $\gamma_{D}$, o sentido do arco, e se o seu centro está à direita ou esquerda da corda AD. 


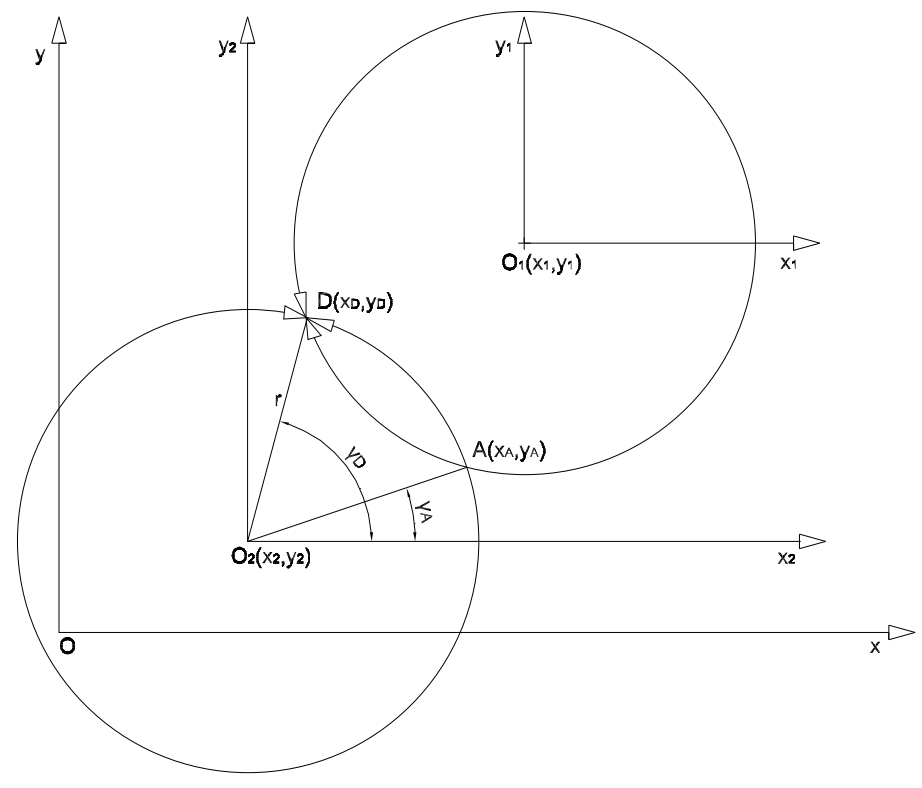

Figura 5.4: Trajetória circular caso geral

O ponto $A$ em relação ao sistema $O$ é igual ao ponto $A$ em relação ao sistema $O_{1}$ (ou $\left.O_{2}\right)$ mais o vetor $\left(O_{1}-O\right)$ (ou $\left(O_{2}-O\right)$ ). O mesmo se aplica para o $D$ ou qualquer outro ponto.

Então para o centro $O_{1}$ podemos escrever:

$$
\begin{aligned}
& { }^{0} A={ }^{1} A+\left(O_{1}-O\right) \\
& { }^{0} D={ }^{1} D+\left(O_{1}-O\right)
\end{aligned}
$$

Para o centro $\mathrm{O}_{2}$ temos:

$$
\begin{aligned}
& { }^{0} A={ }^{2} A+\left(O_{2}-O\right) \\
& { }^{0} D={ }^{2} D+\left(O_{2}-O\right)
\end{aligned}
$$




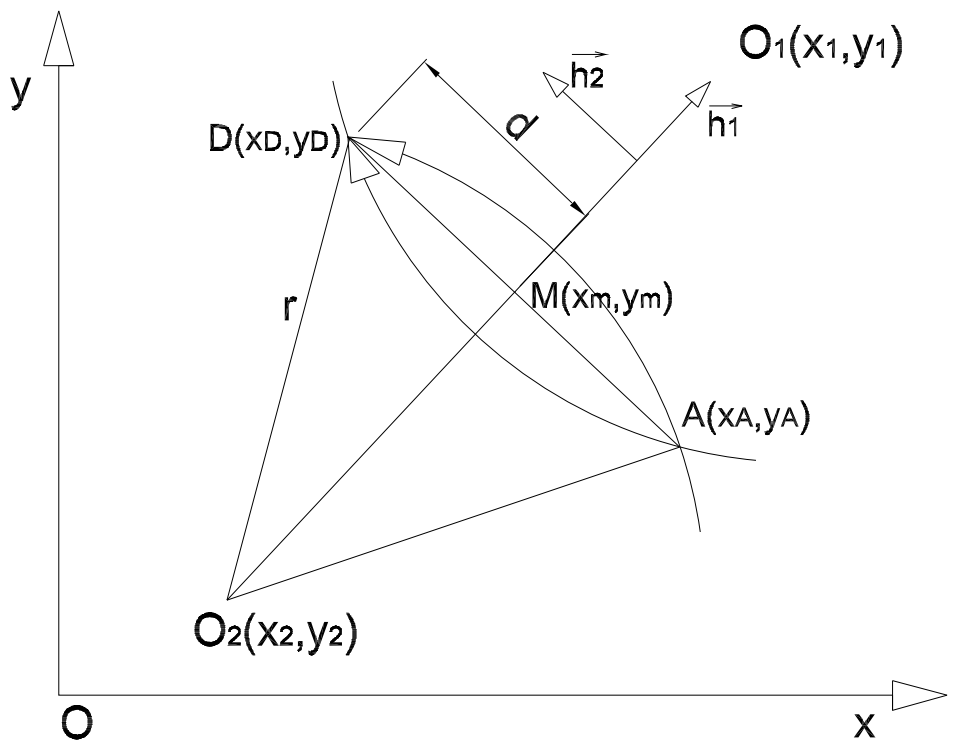

Figura 5.5: Trajetória circular, detalhe

Como relacionamos os pontos com os eixos de coordenadas principal $O$, podemos de agora em diante trabalharmos somente neste sistema.

Chamando de $M\left(x_{m}, y_{m}\right)$ o ponto médio da corda $A D$, de $d$ a metade da corda $A D$, e de $r$ o raio do arco $A D$, como mostrado na fig. 5.5, os centros $O_{1}$ e $O_{2}$, respectivamente à direita e á esquerda da corda $A D$, são definidos pelos vetores:

$$
\begin{aligned}
& \overrightarrow{O_{1}}=\vec{M}+\sqrt{r^{2}-d^{2}} \overrightarrow{h_{1}} \\
& \overrightarrow{O_{2}}=\vec{M}-\sqrt{r^{2}-d^{2}} \overrightarrow{h_{1}}
\end{aligned}
$$

Onde

$$
\begin{gathered}
d=\frac{\|D-A\|}{2} \\
\overrightarrow{h_{2}}=\frac{(D-A)}{\|D-A\|}=h_{2 x}+j h_{2 y}
\end{gathered}
$$

O versor $\overrightarrow{h_{1}}$ é o versor $\overrightarrow{h_{2}}$ rotacionado de $90^{\circ}$, isto é, multiplicado por $j$ :

$$
\overrightarrow{h_{1}}=j\left(h_{2 x}+j h_{2 y}\right)=-h_{2 y}+j h_{2 x}
$$

O vetor $\vec{M}$ é calculado por:

$$
\vec{M}=\vec{A}+d \overrightarrow{h_{2}}
$$


Ou seja,

$$
\left(x_{m}+j y_{m}\right)=\left(x_{A}+j y_{A}\right)+d\left(h_{2 x}+j h_{2 y}\right)
$$

Portanto com esta equação achamos as coordenadas do ponto $M$. Observe que todas as coordenadas se referem ao sistema principal $O$.

Consequentemente ficaram determinados os centros $O_{1}$ e $O_{2}$ dos arcos, bastando apenas definirmos se queremos o da direita $O_{1}$ ou o da esquerda $O_{2} \mathrm{em}$ relação à corda $A D$.

Conhecendo-se $O_{1}$ ficam determinados os vetores $\left(A-O_{1}\right)$ e $\left(D-O_{1}\right)$ e, portanto, ficam também determinados seus argumento $\gamma_{A 1}$ e $\gamma_{D 1}$. A diferença $\Delta \gamma_{1}=\gamma_{D 1}-\gamma_{A 1}$ permite-nos definir qual é o sentido do arco que queremos:

-Sentido anti-horário: $\Delta \gamma_{1}>0$

-Sentido horário: $\quad \Delta \gamma_{1}<0$

Da mesma forma fazemos para o centro $\mathrm{O}_{2}$ :

A diferença $\Delta \gamma_{2}=\gamma_{D 2}-\gamma_{A 2}$ permite-nos definir qual é o sentido do arco que queremos:

-Sentido anti-horário: $\Delta \gamma_{2}>0$

-Sentido horário: $\quad \Delta \gamma_{2}<0$

Como queremos calcular as velocidades e acelerações para vários pontos igualmente espaçados da trajetória, vamos então dividir o ângulo $\Delta \gamma_{1}$ (independentemente se for $\Delta \gamma_{1}$ ou $\Delta \gamma_{2}$ o desenvolvimento é idêntico ) em $n$ ângulos iguais a $\Delta \theta$, isto é,

$$
\Delta \theta=\frac{\Delta \gamma_{1}}{n}
$$

Então para um ponto qualquer $P_{i}$ das subdivisões da trajetória, o vetor $\vec{P}_{i}$ será determinado pela soma do vetor do centro do arco $\overrightarrow{O_{1}}$ mais o vetor $\left(A-O_{1}\right)$ rotacionado de $(i-1) \Delta \theta$, onde o índice $i$ varia de 1 até $n$. Isto é,

$$
\vec{P}_{i}=\left(O_{1}-O\right)+\left(A-O_{1}\right) e^{j \Delta \theta(i-1)}
$$

Então para $i=1$ não há rotação e $P_{1}$ coincide com $A$.

Para $i=n+1$ teremos a rotação $n \Delta \theta=\Delta \gamma_{1}$ e $P_{n+1}$ coincide com $D$.

No anexo $\mathrm{F}$ apresentamos o programa desenvolvido no software Mathematica que calcula as componentes da velocidade e da aceleração para um ponto ou para vários pontos igualmente espaçados da trajetória.

Na elaboração deste programa os centros dos arcos são determinados com as equações:

$$
\left(x_{A}-x_{i}\right)^{2}+\left(y_{A}-y_{i}\right)^{2}=r^{2}
$$




$$
\left(x_{D}-x_{i}\right)^{2}+\left(y_{D}-y_{i}\right)^{2}=r^{2}
$$

Estas duas equações calculam dois centros $\left(x_{i}, y_{i}\right)$ onde $i=1$ ou 2 .

Os ângulos dos raios que dão os pontos inicial e final do arco são determinados por

$$
\begin{aligned}
\gamma_{A} & =\arccos \frac{x_{A}-x_{i}}{r} \\
\gamma_{D} & =\arccos \frac{x_{D}-x_{i}}{r}
\end{aligned}
$$

Definimos no programa que o sentido do arco é positivo quando ele cresce no sentido anti-horário. Para a escolha do centro do arco utilizamos o produto vetorial do vetor que vai do meio da corda $\mathrm{AD}$ até o centro do arco com o vetor que vai de $\mathrm{A}$ até $\mathrm{D}$.

$\mathrm{O}$ anexo $\mathrm{F}$ é utilizado para o caso em que o centro do arco não coincide como a origem dos eixos de coordenadas principal. Nele devemos entrar com o raio do arco, com a aceleração tangencial (módulo), com o ângulo do raio que corresponde ao ponto $\mathrm{P}$ (ponto no qual queremos achar as componentes da velocidade e aceleração) ou com o número de pontos onde queremos achar as componentes da velocidade e aceleração, com as coordenadas cartesianas de A e D, com o sentido (sinal) do arco (horário ou anti-horário) e com a definição se queremos o arco cujo centro está à direita ou esquerda da corda $\mathrm{AD}$.

Neste caso teremos dois resultados:

a) Um vetor com as componentes da velocidade e aceleração para o ponto $P$.

b) $n$ vetores com as componentes da velocidade e aceleração para $n$ pontos intermediários do arco $\mathrm{AD}$. 


\section{Capítulo 6: ANÁLISE CINEMÁTICA: VELOCIDADES E ACELERAÇÕES}

\subsection{Introdução}

Nas equações que desenvolveremos pelo método de Kane no Capítulo 8: Análise dinâmica, necessitaremos dos valores das velocidades e acelerações angulares dos elos, $u_{1}=\dot{\theta}_{1}, \dot{u}_{1}=\ddot{\theta}_{1}, u_{2}=\dot{\theta}_{2}, \dot{u}_{2}=\ddot{\theta}_{2}$, etc.; bem como precisaremos dos valores das acelerações lineares dos centros de gravidade dos elos e suas componentes, isto é, $A_{A}^{n}, A_{A}^{t}, A_{B}^{n}, A_{B}^{t}$, etc. Então vamos adotar a mesma simbologia e nomenclatura que serão usadas naquele capítulo e conforme mostrado na fig.6.1, onde as letras com asterisco indicam a posição do centro de gravidade (CG) dos elos.

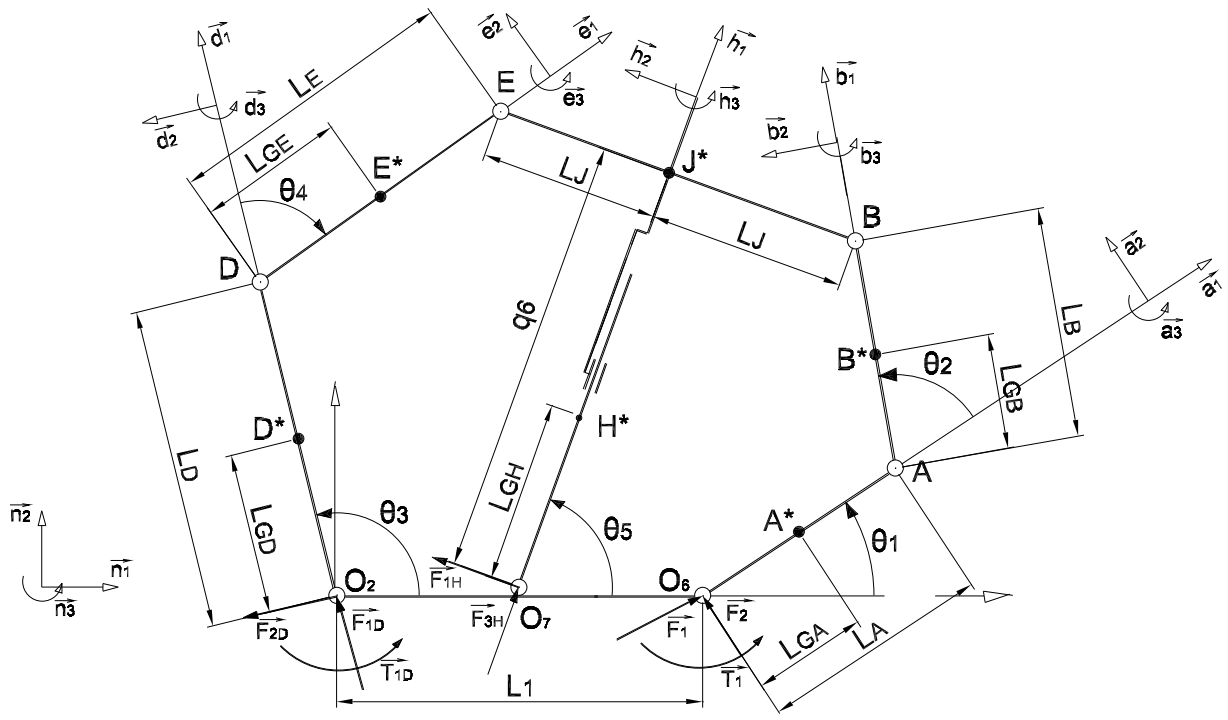

Figura 6.1: Diagrama cinemático do mecanismo

\subsection{Velocidades e acelerações angulares}

Nosso objetivo é a determinação das velocidades $u_{1}, u_{2}, u_{3}, u_{4}, u_{5}, u_{6}$ e das acelerações $\dot{u_{1}}, \dot{u_{2}}, \dot{u_{3}}, \dot{u}_{4}, \dot{u}_{5}$ e $\dot{u}_{6}$, supondo conhecidas a velocidade e aceleração do ponto $J^{*}$ (CG da plataforma móvel) as quais podem ser determinadas conforme o Capítulo 5. Em nosso trabalho consideraremos este ponto como ponto de referência da movimentação do mecanismo. Logicamente o ponto de referência da movimentação do mecanismo vai estar na garra do mecanismo e, portanto, a uma 
certa distância fixa do ponto $J^{*}$. Mas esta é uma simplificação que adotaremos, pois a situação real deste ponto dependerá de cada aplicação prática.

Consideraremos também como sendo dados os ângulos $\theta_{1}, \theta_{2}, \theta_{3}, \theta_{4}, \theta_{5}$ e o deslocamento $q_{6}$ já que os podemos calcular usando as equações da cinemática inversa ou direta que vimos anteriormente no Capítulo 3.

\subsubsection{Cadeia passiva}

Determinação de $u_{5}=\dot{\theta}_{5}$ e $u_{6}=\dot{q}_{6}$.

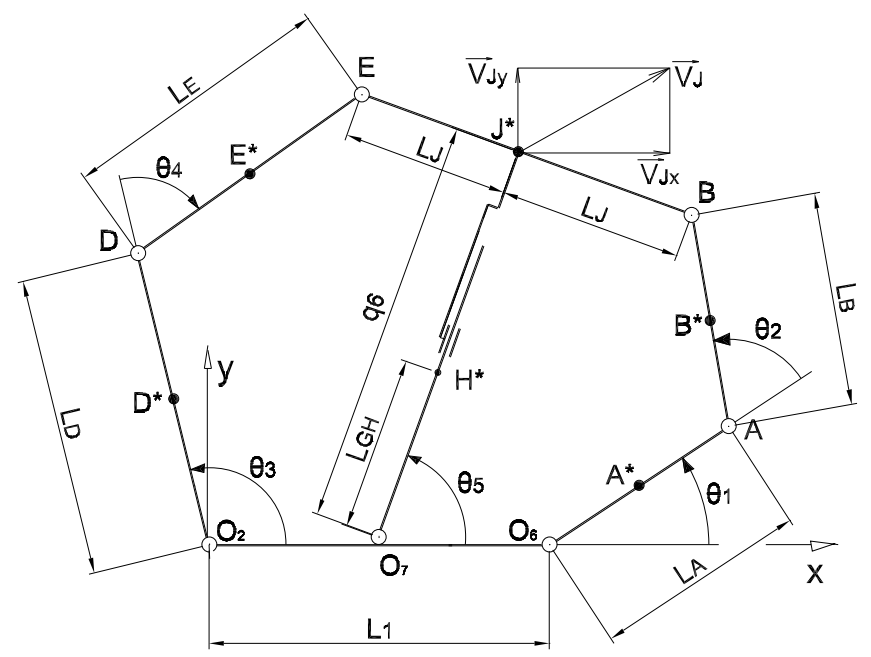

Figura 6.2: Dimensões e ângulos dos elos

Conforme a fig.6.2 vemos que $\left(J^{*}-O_{7}\right)=q_{6} e^{j \theta_{5}}$, portanto, a velocidade linear da garra é $\overrightarrow{V_{J}}=\left(J^{*} \stackrel{\bullet}{-} O_{7}\right)$ isto é:

$$
V_{J x}+j V_{J y}=\dot{q}_{6} e^{j \theta_{5}}+q_{6} j \dot{\theta}_{5} e^{j \theta_{5}}=\dot{q}_{6}\left(c_{5}+j s_{5}\right)+q_{6} \dot{\theta}_{5}\left(-s_{5}+j c_{5}\right)
$$

que desenvolvendo chegamos a

$$
\begin{aligned}
& V_{J x}=c_{5} \dot{q}_{6}-q_{6} s_{5} \dot{\theta}_{5}=c_{5} u_{6}-q_{6} s_{5} u_{5} \\
& V_{J y}=s_{5} \dot{q_{6}}+q_{6} c_{5} \dot{\theta}_{5}=s_{5} u_{6}+q_{6} c_{5} u_{5}
\end{aligned}
$$


Determinação de $\dot{u}_{5}$ e $\dot{u}_{6}$.

As componentes da aceleração linear da garra são $a_{J x}=\dot{V_{J x}}$ e $a_{J y}=\dot{V_{J y}}$ que desenvolvendo chegamos a

$$
a_{J x}=-q_{6} s_{5} \ddot{\theta}_{5}+c_{5} \ddot{q}_{6}-2 \dot{\theta_{5}} \dot{q}_{6} s_{5}-q_{6}\left(\dot{\theta_{5}}\right)^{2} c_{5}
$$

ou seja,

$$
a_{J x}=-q_{6} s_{5} \dot{u}_{5}+c_{5} \dot{u}_{6}-2 u_{5} u_{6} s_{5}-q_{6}\left(u_{5}\right)^{2} c_{5}
$$

Por outro lado

$$
a_{J y}=q_{6} c_{5} \ddot{\theta}_{5}+s_{5} \ddot{q}_{6}+2 \dot{\theta}_{5} \dot{q}_{6} c_{5}-q_{6}\left(\dot{\theta_{5}}\right)^{2} s_{5}
$$

ou seja,

$$
a_{J y}=q_{6} c_{5} \dot{u_{5}}+s_{5} \dot{u_{6}}+2 u_{5} u_{6} c_{5}-q_{6}\left(u_{5}\right)^{2} s_{5}
$$

\subsubsection{Cadeia ativa direita}

Determinação de $u_{1}=\dot{\theta}_{1} \mathbf{e} u_{2}=\dot{\theta}_{2}$

Conforme a fig. 6.2 podemos escrever:

$$
\begin{aligned}
& \left(J^{*}-O_{7}\right)=\left(O_{6}-O_{7}\right)+\left(A-O_{6}\right)+(B-A)+\left(J^{*}-B\right) \\
& \left(J^{*}-O_{7}\right)=\left(O_{6}-O_{7}\right)+L_{A} e^{j \theta_{1}}+L_{B} e^{j\left(\theta_{1}+\theta_{2}\right)}+L_{J} j e^{j \theta_{5}}
\end{aligned}
$$

Mas

$$
\overrightarrow{V_{J}}=\left(J^{*} \stackrel{\bullet}{-} O_{7}\right)
$$

então

$$
\overrightarrow{V_{J}}=L_{A} j \dot{\theta_{1}} e^{j \theta_{1}}+L_{B} j\left(\dot{\theta_{1}}+\dot{\theta_{2}}\right) e^{j\left(\theta_{1}+\theta_{2}\right)}-L_{J} \dot{\theta}_{5} e^{j \theta_{5}}
$$

que desenvolvendo chegamos a

$$
V_{J x}=-L_{A} s_{1} \dot{\theta}_{1}-L_{B} s_{12}\left(\dot{\theta_{1}}+\dot{\theta}_{2}\right)-L_{J} c_{5} \theta_{5}
$$

isto é,

$$
V_{J x}=-L_{A} s_{1} u_{1}-L_{B} s_{12}\left(u_{1}+u_{2}\right)-L_{J} c_{5} u_{5}
$$


Por outro lado temos

$$
V_{J y}=L_{A} c_{1} \dot{\theta_{1}}+L_{B} c_{12}\left(\dot{\theta_{1}}+\dot{\theta_{2}}\right)-L_{J} s_{5} \dot{\theta_{5}}
$$

isto é,

$$
V_{J y}=L_{A} c_{1} u_{1}+L_{B} c_{12}\left(u_{1}+u_{2}\right)-L_{J} s_{5} u_{5}
$$

\section{Determinação de $\dot{u_{1}}$ e $\dot{u_{2}}$}

Sabemos que $a_{J x}=V_{J x}$ e $a_{J y}=V_{J y}$ então desenvolvendo chegamos a

$$
\begin{gathered}
a_{J x}=-L_{A} c_{1}\left(\dot{\theta_{1}}\right)^{2}-L_{A} s_{1} \ddot{\theta}_{1}-L_{B} c_{12}\left(\dot{\theta_{1}}+\dot{\theta}_{2}\right)^{2}- \\
L_{B} s_{12}\left(\ddot{\theta_{1}}+\ddot{\theta_{2}}\right)+L_{J} s_{5}\left(\dot{\theta}_{5}\right)^{2}-L_{J} c_{5} \ddot{\theta}_{1}
\end{gathered}
$$

ou seja,

$$
\begin{gathered}
a_{J x}=-L_{A} c_{1}\left(u_{1}\right)^{2}-L_{A} s_{1} \dot{u_{1}}-L_{B} c_{12}\left(u_{1}+u_{2}\right)^{2}- \\
L_{B} s_{12}\left(\dot{u_{1}}+\dot{u_{2}}\right)+L_{J} s_{5}\left(u_{5}\right)^{2}-L_{J} c_{5} \dot{u_{5}}
\end{gathered}
$$

Por outro lado,

$$
\begin{gathered}
a_{J y}=-L_{A} s_{1}\left(\dot{\theta_{1}}\right)^{2}+L_{A} c_{1} \ddot{\theta}_{1}-L_{B} s_{12}\left(\dot{\theta}_{1}+\dot{\theta_{2}}\right)^{2}+ \\
L_{B} c_{12}\left(\ddot{\theta_{1}}+\ddot{\theta_{2}}\right)-L_{J} c_{5}\left(\dot{\theta}_{5}\right)^{2}-L_{J} s_{5} \ddot{\theta}_{1}
\end{gathered}
$$

ou seja,

$$
\begin{gathered}
a_{J y}=-L_{A} s_{1}\left(u_{1}\right)^{2}+L_{A} c_{1} \dot{u_{1}}-L_{B} s_{12}\left(u_{1}+u_{2}\right)^{2}+ \\
L_{B} c_{12}\left(\dot{u_{1}}+\dot{u_{2}}\right)-L_{J} c_{5}\left(u_{5}\right)^{2}-L_{J} s_{5} \dot{u_{5}}
\end{gathered}
$$

\subsubsection{Cadeia ativa esquerda}

Determinação de $u_{3}=\dot{\theta}_{3}$ e $u_{4}=\dot{\theta}_{4}$.

Mutatis mutandis em relação à cadeia ativa direita, podemos escrever:

$$
V_{J x}=-L_{D} s_{3} u_{3}-L_{E} s_{34}\left(u_{3}+u_{4}\right)+L_{J} c_{5} u_{5}
$$




$$
V_{J y}=L_{D} c_{3} u_{3}+L_{E} c_{34}\left(u_{3}+u_{4}\right)+L_{J} s_{5} u_{5}
$$

Determinação de $\dot{u_{3}}$ e $\dot{u}_{4}$.

Mutatis mutandis em relação à cadeia ativa direita, podemos escrever:

$$
\begin{gathered}
a_{J x}=-L_{D} c_{3}\left(u_{3}\right)^{2}-L_{D} s_{3} \dot{u_{3}}-L_{E} c_{34}\left(u_{3}+u_{4}\right)^{2}- \\
L_{E} s_{34}\left(\dot{u} 3+\dot{u_{4}}\right)-L_{J} s_{5}\left(u_{5}\right)^{2}+L_{J} c_{5} \dot{u_{5}} \\
a_{J y}=-L_{D} s_{3}\left(u_{3}\right)^{2}+L_{D} c_{3} \dot{u_{3}}-L_{E} s_{34}\left(u_{3}+u_{4}\right)^{2}+ \\
L_{E} c_{34}\left(\dot{u_{3}}+\dot{u_{4}}\right)-L_{J} c_{5}\left(u_{5}\right)^{2}-L_{J} s_{5} \dot{u_{5}}
\end{gathered}
$$

No Anexo $\mathrm{G}$ apresentamos o programa Mathematica que, utilizando as equações acima, deduz as equações para o cálculo das velocidades $\theta_{1}=u_{1}, \theta_{2}=u_{2}, \theta_{3}=u_{3}$, $\dot{\theta}_{4}=u_{4}, \dot{\theta}_{5}=u_{5}$ e $\dot{q}_{6}=u_{6}$ e as acelerações $\ddot{\theta}_{1}=\dot{u}_{1}, \ddot{\theta}_{2}=\dot{u}_{2}, \ddot{\theta}_{3}=\dot{u}_{3}, \ddot{\theta}_{4}=\dot{u}_{4}$, $\ddot{\theta}_{5}=\dot{u}_{5}$ e $\ddot{q}_{6}=\dot{u}_{6}$. O Anexo $\mathrm{H}$ calcula seus valores numéricos.

\subsection{Acelerações dos CG dos elos}

6.3.1 Elo A: $A_{A}^{n}$ e $A_{A}^{t}$

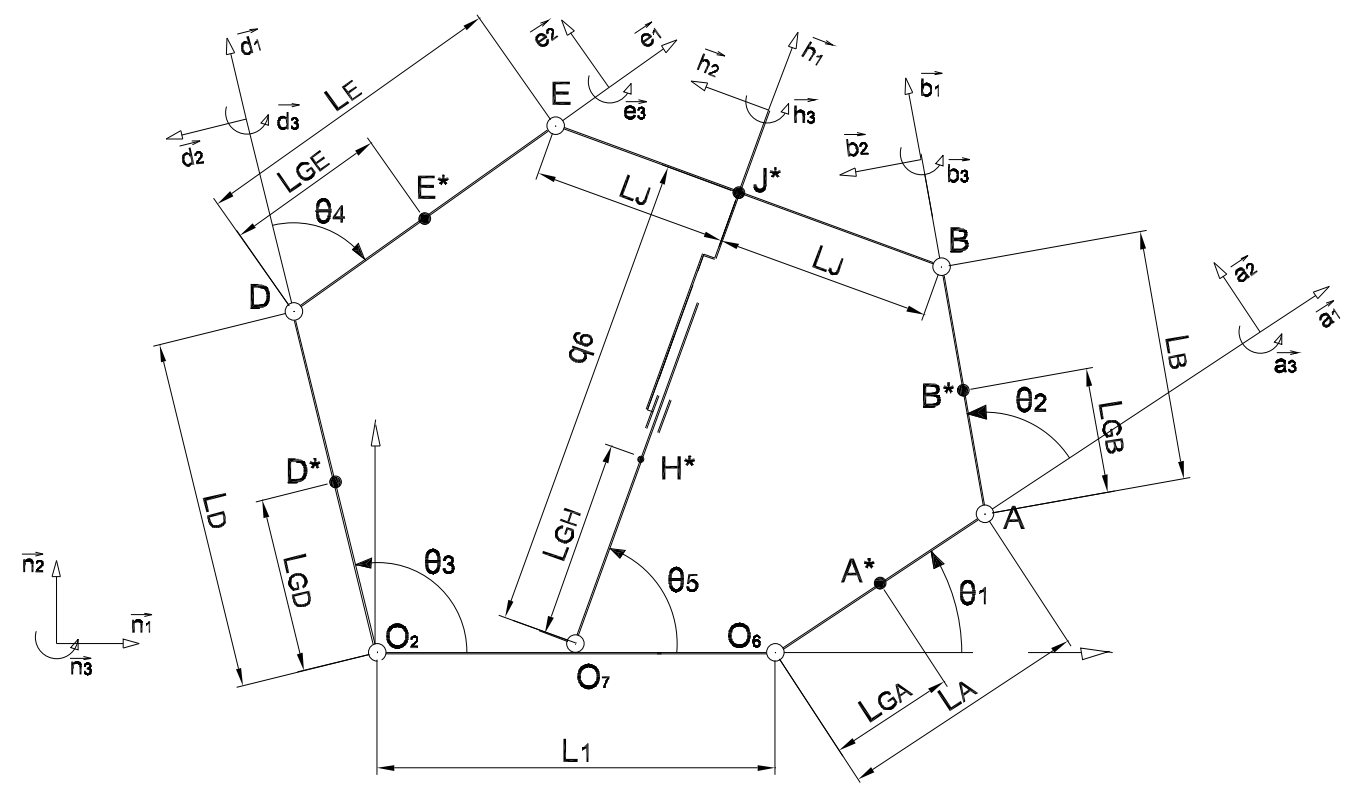

Figura 6.3: Diagrama auxiliar para determinação das acelerações 
$A_{A}^{n}$ é a componente da aceleração linear do ponto $A^{*}$ (CG do elo $\left.A\right)$ na direção $\overrightarrow{a_{1}}$, enquanto que $A_{A}^{t}$ é a componente da mesma aceleração na direção $\overrightarrow{a_{2}}$ (ver fig. 6.3).

Temos

$$
\overrightarrow{A_{A}}=\frac{d \overrightarrow{V_{A}}}{d t}
$$

e sendo

$$
\overrightarrow{V_{A}}=L_{G A} u_{1} \overrightarrow{a_{2}}
$$

então

$$
\overrightarrow{A_{A}}=L_{G A} \dot{u_{1}} \overrightarrow{a_{2}}-L_{G A}\left(u_{1}\right)^{2} \overrightarrow{a_{1}}
$$

Isto é

$$
\begin{gathered}
A_{A}^{n}=-L_{G A}\left(u_{1}\right)^{2} \\
A_{A}^{t}=L_{G A} \dot{u_{1}}
\end{gathered}
$$

\subsubsection{Elo B: $A_{B}^{n}$ e $A_{B}^{t}$}

$A_{B}^{n}$ é a componente da aceleração linear do ponto $B^{*}(\mathrm{CG}$ do elo $B)$ na direção $\overrightarrow{b_{1}}$, enquanto que $A_{B}^{t}$ é a componente da mesma aceleração na direção $\overrightarrow{b_{2}}$.

Temos então

$$
\overrightarrow{A_{B}}=\frac{d \overrightarrow{V_{B}}}{d t}
$$

e sendo

$$
\overrightarrow{V_{B}}=L_{A} u_{1} \overrightarrow{a_{2}}+L_{G B}\left(u_{1}+u_{2}\right) \overrightarrow{b_{2}}
$$

então

$$
\begin{gathered}
\overrightarrow{A_{B}}=L_{A} \dot{u_{1}} \overrightarrow{a_{2}}-L_{G A}\left(u_{1}\right)^{2} \overrightarrow{a_{1}}+L_{G B}\left(\dot{u_{1}}+\dot{u_{2}}\right)\left(-s_{12} \overrightarrow{n_{1}}+c_{12} \overrightarrow{n_{2}}\right)- \\
L_{G B}\left(u_{1}+u_{2}\right)^{2}\left(c_{12} \overrightarrow{n_{1}}+s_{12} \overrightarrow{n_{2}}\right)
\end{gathered}
$$

Mas $A_{B}^{n}=\overrightarrow{A_{B}} \cdot \overrightarrow{b_{1}}$.

Fazendo este produto escalar e desenvolvendo chegamos a

$$
A_{B}^{n}=L_{A} \dot{u_{1}} s_{2}-L_{A}\left(u_{1}\right)^{2} c_{2}-L_{G B}\left(u_{1}+u_{2}\right)^{2}
$$


Por outro lado $A_{B}^{t}=\overrightarrow{A_{B}} \cdot \overrightarrow{b_{2}}$.

Fazendo este produto escalar e desenvolvendo chegamos a

$$
A_{B}^{t}=L_{A} \dot{u_{1}} c_{2}-L_{A}\left(u_{1}\right)^{2} s_{2}+L_{G B}\left(\dot{u_{1}}+\dot{u_{2}}\right)
$$

6.3.3 Elo D: $A_{D}^{n}$ e $A_{D}^{t}$

Mutatis mutandis em relação ao elo A temos:

$$
\begin{gathered}
A_{D}^{n}=-L_{G D}\left(u_{3}\right)^{2} \\
A_{D}^{t}=L_{G D} \dot{u_{3}}
\end{gathered}
$$

6.3.4 Elo E: $A_{E}^{n}$ e $A_{E}^{t}$

Mutatis mutandis em relação ao elo B temos:

$$
\begin{aligned}
& A_{E}^{n}=L_{D} \dot{u_{3}} s_{4}-L_{D}\left(u_{3}\right)^{2} c_{4}-L_{G E}\left(u_{3}+u_{4}\right)^{2} \\
& A_{E}^{t}=L_{D} \dot{u_{3}} c_{4}-L_{D}\left(u_{3}\right)^{2} s_{4}+L_{G E}\left(\dot{u_{3}}+\dot{u_{4}}\right)
\end{aligned}
$$

\subsubsection{Elo H: $A_{H}^{n}$ e $A_{H}^{t}$}

Temos

$$
\begin{gathered}
\overrightarrow{V_{H}}=L_{G H} \overrightarrow{h_{1}}=L_{G H} u_{5} \overrightarrow{h_{2}} \\
\overrightarrow{A_{H}}=\frac{d \overrightarrow{V_{H}}}{d t}
\end{gathered}
$$

que desenvolvendo chegamos a

$$
\overrightarrow{A_{H}}=-L_{G H}\left(u_{5}\right)^{2} \overrightarrow{h_{1}}+L_{G H} \dot{u_{5}} \overrightarrow{h_{2}}
$$

Portanto concluímos

$$
\begin{gathered}
A_{H}^{n}=-L_{G H}\left(u_{5}\right)^{2} \\
A_{H}^{t}=L_{G H} \dot{u_{5}}
\end{gathered}
$$

6.3.6 Elo J: $A_{J}^{n}$ e $A_{J}^{t}$

Temos

$$
\overrightarrow{A_{J}}=\frac{d \overrightarrow{V_{J}}}{d t}
$$


e sendo

$$
\overrightarrow{V_{J}}=u_{6} \overrightarrow{h_{1}}+q_{6} u_{5} \overrightarrow{h_{2}}
$$

então

$$
\overrightarrow{A_{J}}=\left(\dot{u_{6}}-q_{6}\left(u_{5}\right)^{2}\right) \overrightarrow{h_{1}}+\left(2 u_{6} u_{5}+q_{6} \dot{u_{5}}\right) \overrightarrow{h_{2}}
$$

portanto, concluímos

$$
\begin{aligned}
& A_{J}^{t}=\dot{u_{6}}-q_{6}\left(u_{5}\right)^{2} \\
& A_{J}^{n}=2 u_{6} u_{5}+q_{6} \dot{u_{5}}
\end{aligned}
$$

No Anexo $\mathrm{H}$ apresentamos o programa elaborado no software Mathematica que com as equações 6.1 a 6.12 determina os valores numéricos das velocidades $\dot{\theta}_{1}=u_{1}, \dot{\theta}_{2}=u_{2}, \dot{\theta}_{3}=u_{3}, \dot{\theta}_{4}=u_{4}, \dot{\theta}_{5}=u_{5}$ e $\dot{q}_{6}=u_{6}$ e das acelerações $\ddot{\theta}_{1}=\dot{u}_{1}$, $\ddot{\theta}_{2}=\dot{u}_{2}, \ddot{\theta}_{3}=\dot{u}_{3}, \ddot{\theta}_{4}=\dot{u}_{4}, \ddot{\theta}_{5}=\dot{u}_{5}$ e $\ddot{q}_{6}=\dot{u}_{6}$; e com as equações 6.13 a 6.24 determina as acelerações dos $\mathrm{CG}$ dos elos, isto é, $A_{A}^{n}, A_{A}^{t}, A_{B}^{n}, A_{B}^{t}, A_{D}^{n}, A_{D}^{t}, A_{E}^{n}$, $A_{E}^{t}, A_{H}^{n}, A_{H}^{t}, A_{J}^{n}$ e $A_{J}^{t}$. 


\section{Capítulo 7: ANÁLISE ESTÁTICA}

\subsection{Introdução}

Como a finalidade do mecanismo que estamos estudando é ser utilizado como manipulador robótico, vamos considerar o ponto $G$ (ponto central da plataforma móvel) como ponto de referência da garra, ou seja, todo esforço de manipulação do mecanismo será concentrado neste ponto.

Conforme ilustrado na fig.7.1 vamos considerar que no ponto $G$ atua um momento $\vec{M}$ e a força de manipulação $\vec{F}$, a qual tem uma inclinação $\beta$ em relação à horizontal.

Nesta situação, $\vec{M}$ e $\vec{F}$ darão origem às reações de momento e força nos apoios $\mathrm{O}_{2}$ e $\mathrm{O}_{6}$, onde estão os atuadores. No apoio $O_{7}$ haverá somente reação de força, pois neste ponto existe uma junta de rotação sem atuador.

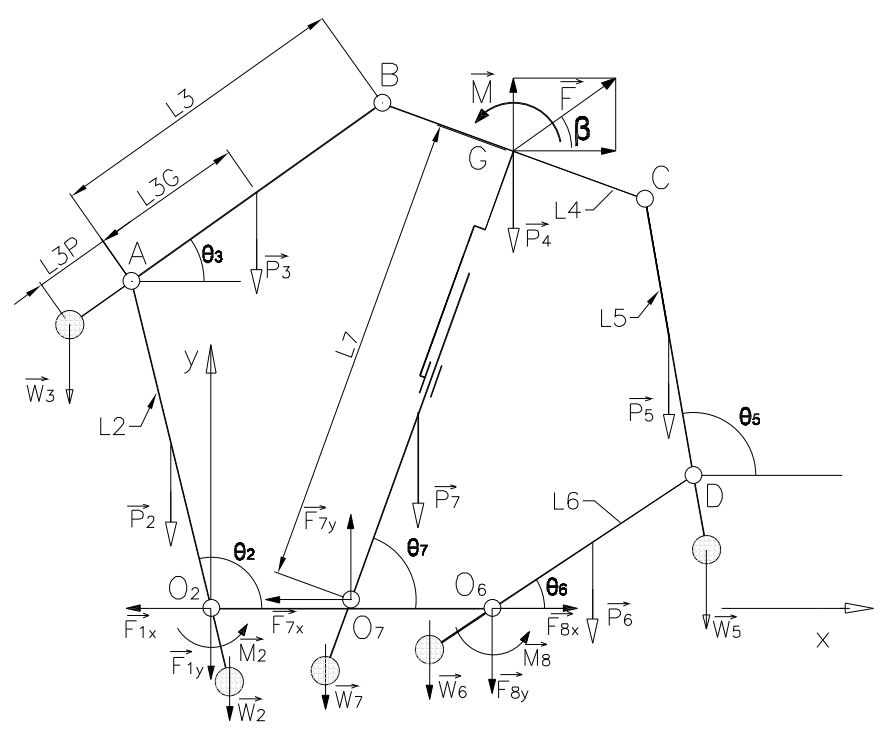

Figura 7.1: Forças e momentos

Para a determinação dos torques nos atuadores e das forças de reação vamos construir os diagramas de corpo livre de cada elo e aplicar as equações de equilíbrio da estática. Os pesos próprios dos elos serão representados por $\vec{P}_{i}$ e seus comprimentos $L_{i}$, sendo $i=1, \ldots, 7$ respectivamente os números das peças. Consideraremos também que os centros de gravidade dos elos estarão situados a uma distância $L_{i G}$ de sua extremidade inferior, exceto no caso da plataforma móvel que o consideraremos situado no ponto $G$. A fim de generalizarmos um pouco 
mais nosso estudo vamos introduzir também os contrapesos $\vec{W}_{i}$ distanciados $L_{i P}$ das juntas dos elos conforme mostram as figuras seguintes.

\subsection{Equações de equilíbrio}

\section{Elo 7}

O diagrama de corpo livre do elo 7 é mostrado na fig.7.2 e as equações escalares de equilíbrio são:

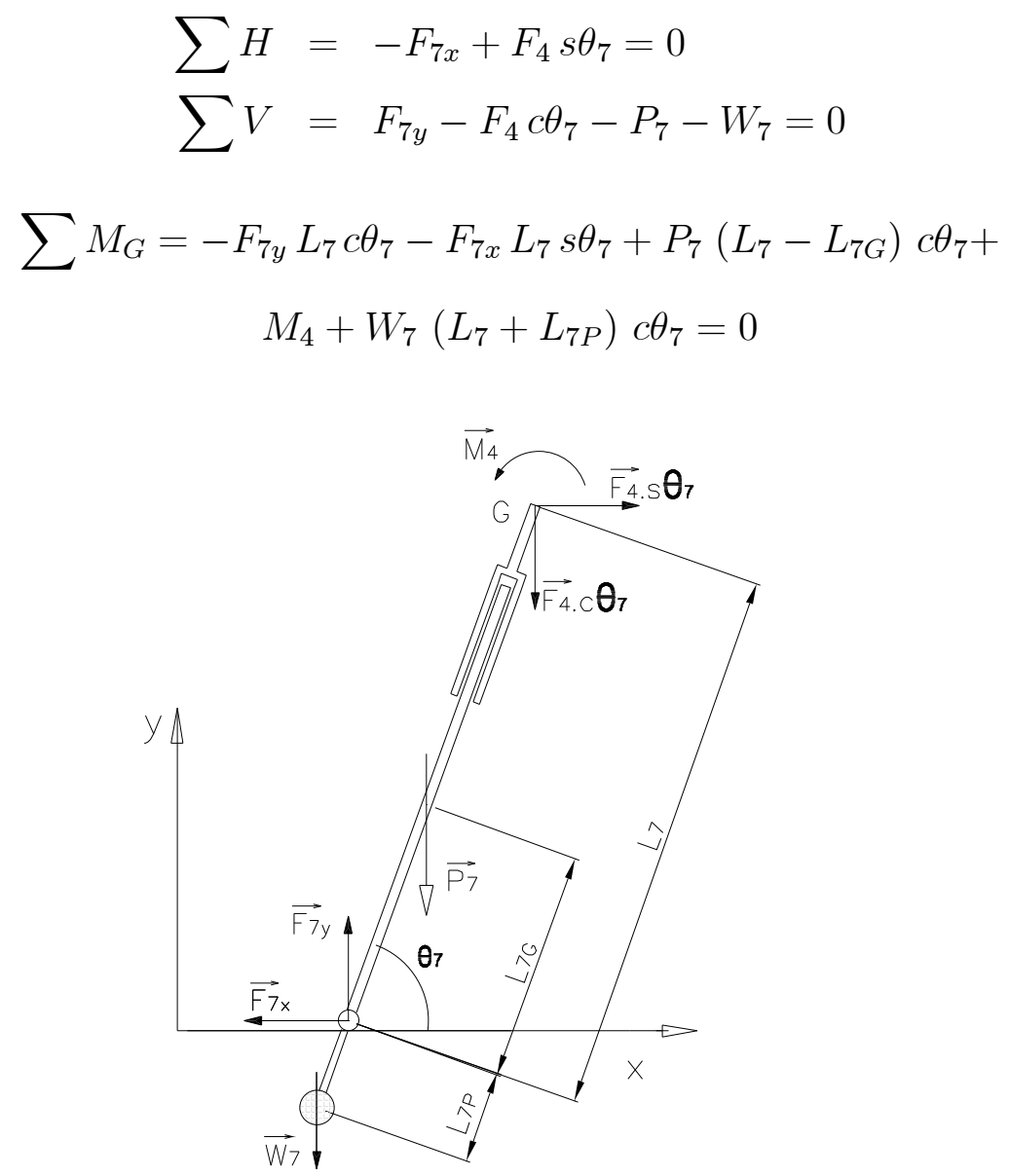

Figura 7.2: Diagrama de corpo livre: elo 7

\section{Elo 4}

O diagrama de corpo livre do elo 4 é mostrado na fig.7.3 e as equações escalares de equilíbrio são: 


$$
\begin{gathered}
\sum H=-F_{3 x}-F_{4} s \theta_{7}+F c \beta+F_{5 x}=0 \\
\sum V=-F_{3 y}+F_{4} c \theta_{7}+F s \beta-F_{5 y}-P_{4}=0 \\
\sum M_{B}=F_{5 x} L_{4} c \theta_{7}-F_{5 y} L_{4} s \theta_{7}+\left(F c \beta-F_{4} s \theta_{7}\right) \frac{L_{4}}{2} c \theta_{7}+ \\
\left(F s \beta+F_{4} c \theta_{7}-P_{4}\right) \frac{L_{4}}{2} s \theta_{7}-M_{4}+M=0
\end{gathered}
$$

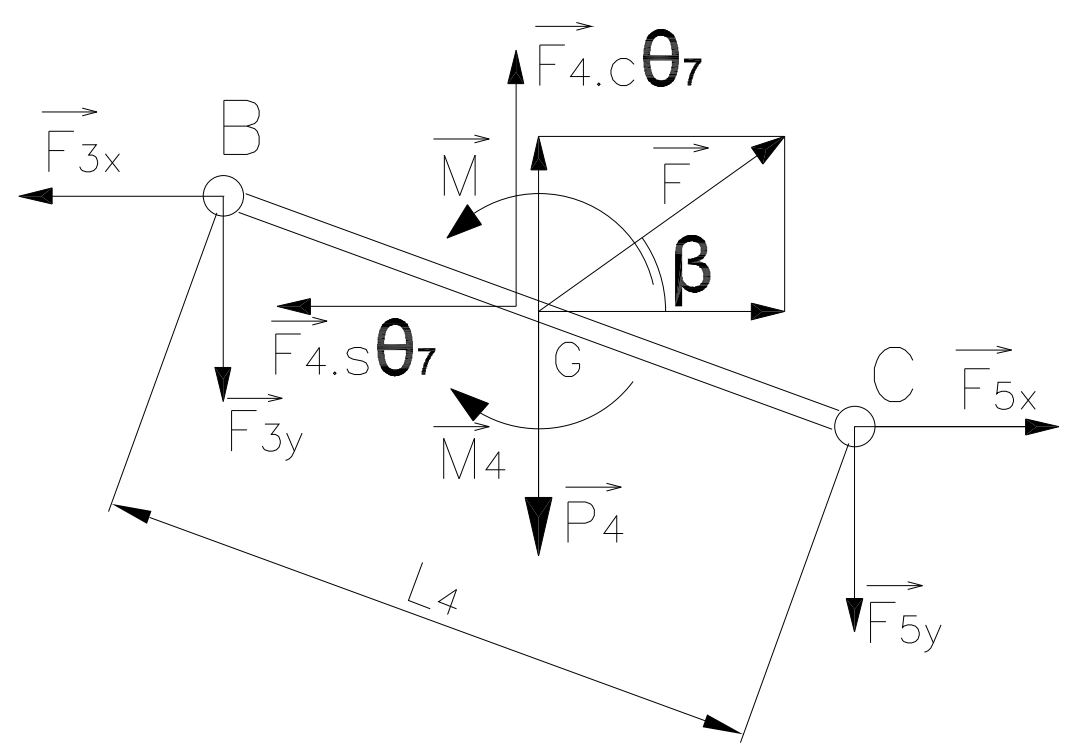

Figura 7.3: Diagrama de corpo livre: elo 4

\section{Elo 3}

O diagrama de corpo livre do elo 3 é mostrado na fig.7.4 e as equações escalares de equilíbrio são:

$$
\begin{aligned}
\sum H & =-F_{2 x}+F_{3 x}=0 \\
\sum V & =-F_{2 y}+F_{3 y}-P_{3}-W_{3}=0 \\
\sum M_{B} & =F_{2 y} L_{3} c \theta_{3}-F_{2 x} L_{3} s \theta_{3}+P_{3}\left(L_{3}-L_{3 G}\right) c \theta_{3}+W_{3}\left(L_{3}+L_{3 P}\right) c \theta_{3}=0
\end{aligned}
$$




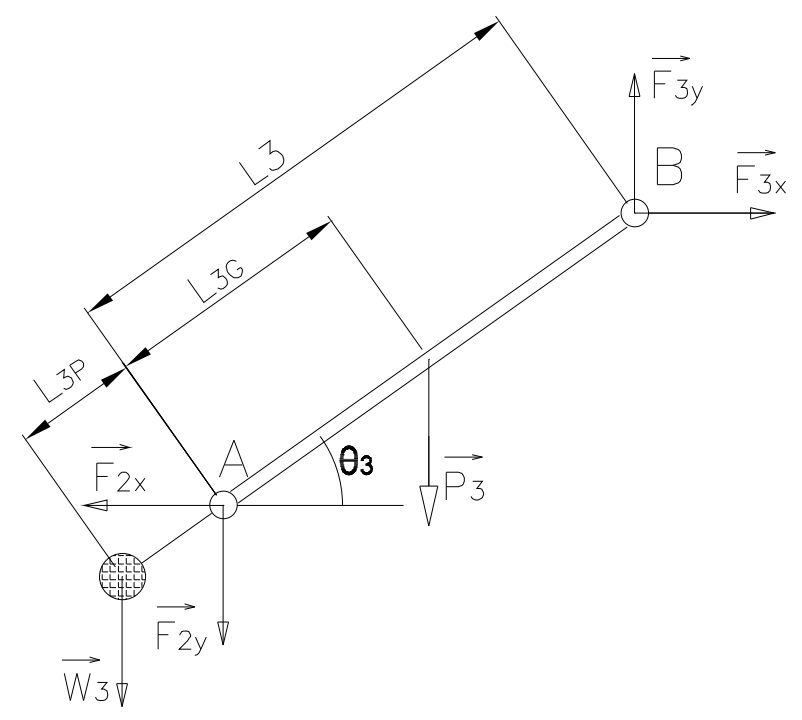

Figura 7.4: Diagrama de corpo livre: elo 3

\section{Elo 2}

O diagrama de corpo livre do elo 2 é mostrado na fig.7.5 e as equações escalares de equilíbrio são:

$$
\begin{aligned}
\sum H & =F_{2 x}-F_{1 x}=0 \\
\sum V & =F_{2 y}-F_{1 y}-P_{2}-W_{2}=0 \\
\sum M_{A} & =F_{1 y} L_{2} c \theta_{2}-F_{1 x} L_{2} s \theta_{2}+P_{2}\left(L_{2}-L_{2 G}\right) c \theta_{2}+M_{2}+W_{2}\left(L_{2}+L_{2 P}\right) c \theta_{2}=0
\end{aligned}
$$




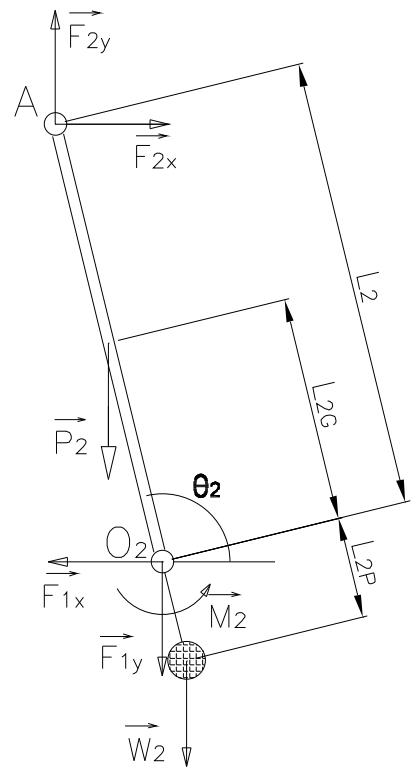

Figura 7.5: Diagrama de corpo livre: elo 2

\section{Elo 5}

O diagrama de corpo livre do elo 5 é mostrado na fig.7.6 e as equações escalares de equilíbrio são:

$$
\begin{aligned}
\sum H & =-F_{5 x}+F_{6 x}=0 \\
\sum V & =F_{5 y}-F_{6 y}-P_{5}-W_{5}=0 \\
\sum M_{C} & =F_{6 y} L_{5} c \theta_{5}+F_{6 x} L_{5} s \theta_{5}+P_{5}\left(L_{5}-L_{5 G}\right) c \theta_{5}+W_{5}\left(L_{5}+L_{5 P}\right) c \theta_{5}=0
\end{aligned}
$$




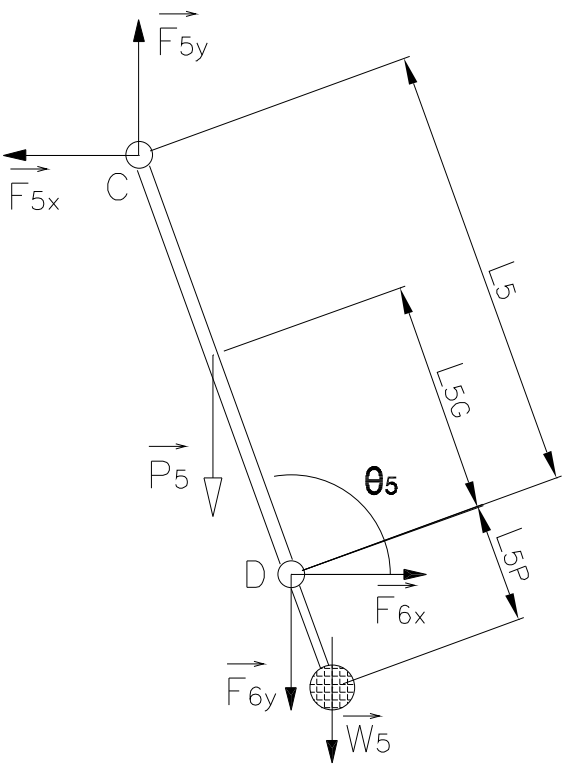

Figura 7.6: Diagrama de corpo livre: elo 5

\section{Elo 6}

O diagrama de corpo livre do elo 6 é mostrado na fig.7.7 e as equações escalares de equilíbrio são:

$$
\begin{aligned}
\sum H & =-F_{6 x}+F_{8 x}=0 \\
\sum V & =F_{6 y}-F_{8 y}-P_{6}-W_{6}=0 \\
\sum M_{B} & =F_{8 y} L_{6} c \theta_{6}+F_{8 x} L_{6} s \theta_{6}+P_{6}\left(L_{6}-L_{6 G}\right) c \theta_{6}+M_{8}+W_{6}\left(L_{6}+L_{6 P}\right) c \theta_{6}=0
\end{aligned}
$$




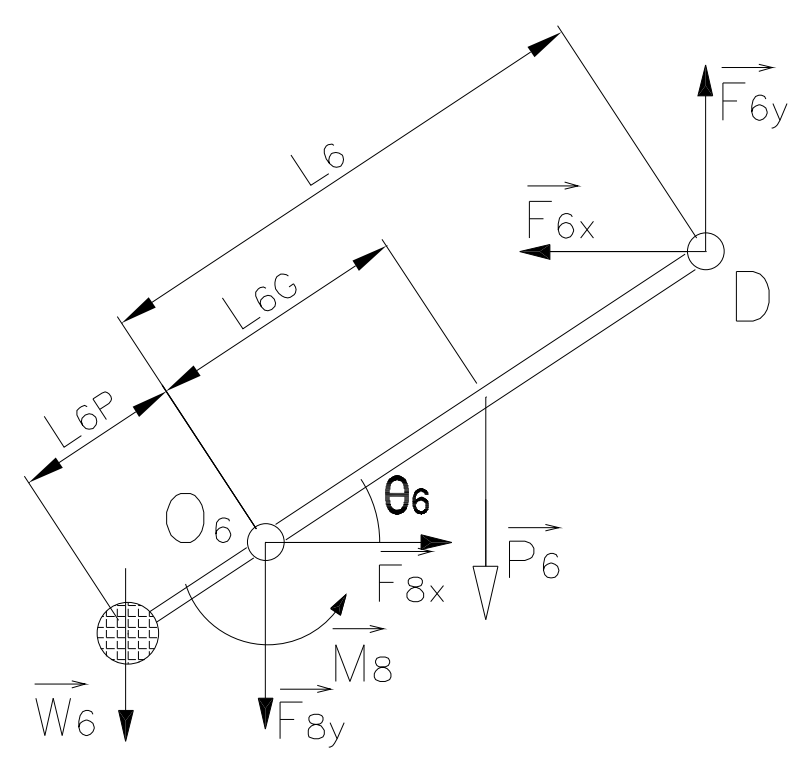

Figura 7.7: Diagrama de corpo livre: elo 6

\subsubsection{Listagem do programa}

De posse destas equações elaboramos um programa no software Mathematica apresentado no Anexo I, o qual foi desenvolvido para a determinação dos torques nos atuadores e das forças de reação, considerando-se que conhecemos a configuração do mecanismo, os ângulos de inclinação dos elos, os comprimento dos elos, seus pesos próprios, as posições dos centros de gravidade, os contrapesos, suas posições, a força externa e momento externo na garra.

\subsection{Determinação dos contrapesos para momen- tos de reação nulos}

Nosso objetivo agora é determinarmos os valores dos contrapesos que anulem estaticamente os torques nos atuadores quando consideramos somente os pesos próprios dos elos. Neste caso vamos estabelecer que a distância dos contrapesos até a articulação seja igual a $1 / 4$ do comprimento do respectivo elo. Vamos considerar também que os centros de gravidade dos elos estejam situados no meio dos seus comprimentos.

$\mathrm{Na}$ fig.7.8 isolamos os elementos do mecanismo com seus respectivos contrapesos para podermos calcular os valores dos contrapesos que equilibram os pesos próprios dos elos. 
-Elo 7.

$$
W_{7}\left(\frac{L_{7}}{4}\right) c \theta_{7}=P_{7}\left(\frac{L_{7}}{2}\right) c \theta_{7}
$$

donde

$$
W_{7}=2 P_{7}
$$
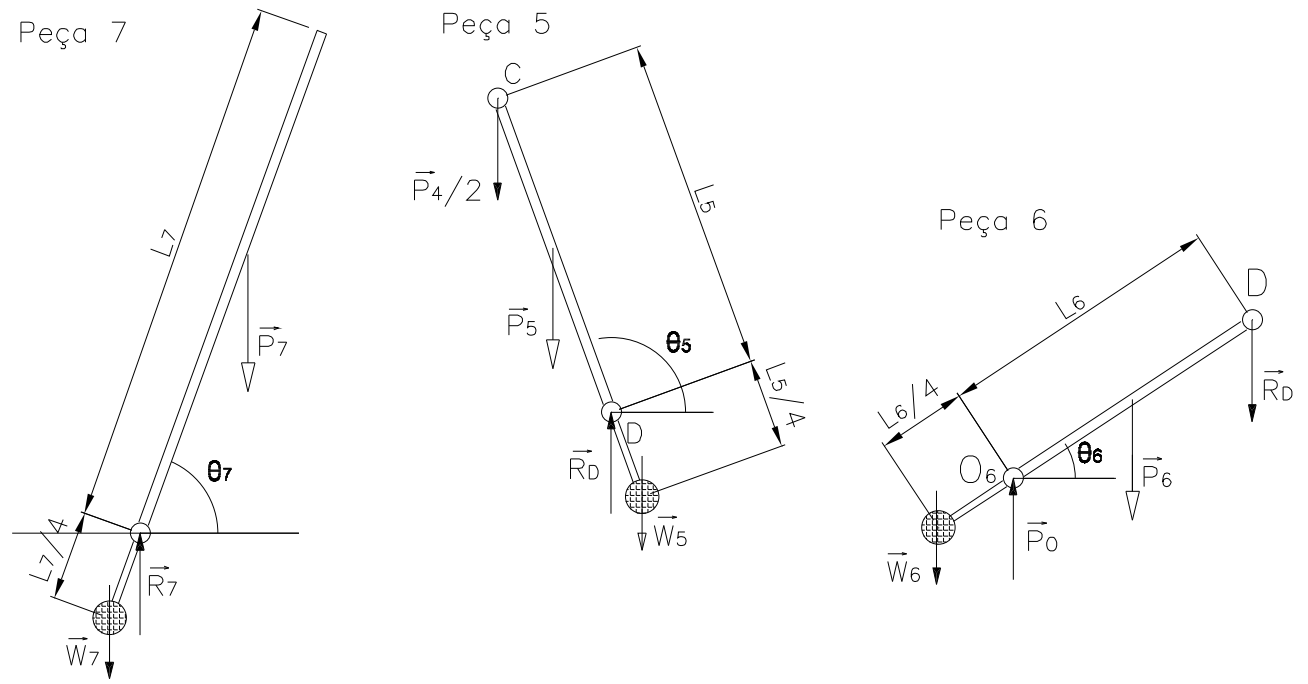

Figura 7.8: Determinação dos contrapesos

-Elo 5

$$
W_{5} \frac{L_{5}}{4}=P_{5} \frac{L_{5}}{2}+\frac{P_{4}}{2} L_{5}
$$

então

$$
W_{5}=2 P_{5}+2 P_{4}
$$

$\mathrm{e}$

$$
R_{D}=\frac{P_{4}}{2}+P_{5}+W_{5}
$$

então

$$
R_{D}=\frac{5}{2} P_{4}+3 P_{5}
$$

-Elo 6

$$
W_{6} \frac{L_{6}}{4}=P_{6} \frac{L_{6}}{2}+R_{D} L_{6}
$$

então

$$
W_{6}=2 P_{6}+10 P_{4}+12 P_{5}
$$


Considerando a simetria do mecanismo podemos colocar:

$$
\begin{aligned}
& W_{3}=2 P_{3}+2 P_{4} \\
& W_{2}=2 P_{2}+12 P_{3}+10 P_{4}
\end{aligned}
$$

Utilizando, portanto, os contrapesos calculados de acordo com as equações acima e empregando o programa do Anexo I podemos verificar que anulamos os momentos de reação, isto, logicamente sem considerar o efeito dinâmico e carga externa.

O balanceamento estático usando contrapesos elimina os momentos de reação, mas em contra partida, aumenta o efeito dinâmico devido ao aumento da inércia. Portanto para cada caso deve-se verificar a vantagem ou não de seu uso. 


\section{Capítulo 8: ANÁLISE DINÂMICA}

\subsection{Introdução}

O desenvolvimento da análise dinâmica será feito, como já dissemos, através do método de Kane, portanto, nesta Introdução apresentaremos algumas noções teóricas sobre este método. Em seguida faremos o desenvolvimento deste método para o caso específico do mecanismo objeto deste trabalho. Descreveremos e mostraremos então um diagrama deste mecanismo com seus parâmetros e versores de referência. Seguem-se as especificações das variáveis, o desenvolvimento das equações que o método de Kane fornece, um roteiro de cálculo e três exemplos de aplicação.

A fim de termos uma noção deste método, apresentaremos no parágrafo seguinte, de forma resumida, alguns tópicos abordados por R. Brent Gillespie em [18] :

No método de Kane as variáveis de movimento $u_{r}(r=1, \ldots, M)$ são em geral funções lineares das variáveis de movimento de Lagrange $\dot{q}_{r}(r=1, \ldots, l)$ e o número de variáveis de movimento de Kane $(M)$ é igual ao número de variáveis de movimento de Lagrange $(l)$ menos o número de vínculos de movimento $(\mathrm{m})$, isto é, $M=l-m$. Portanto, o uso das variáveis de movimento de Kane (também chamadas de generalized speeds em contra-partida às variáveis de movimento de Lagrange denominadas de generalized velocities) geralmente produz equações compactas. No caso de sistemas vinculados não holomicamente o emprego das variáveis de movimento de Kane leva-nos a uma equação diferencial ordinária enquanto que, quando usamos as variáveis de movimento de Lagrange, precisamos usar o método dos multiplicadores independentes.

Para deduzir as equações de Kane vamos considerar um sistema com $n$ corpos $P i,(i=1,2, \ldots, n)$. Seja $\vec{R}_{i}$ a resultante das forças que agem no corpo $P_{i}$ e aplicada em seu centro de gravidade. Seja $\overrightarrow{T_{i}}$ a soma de todos os momentos aplicados em $P_{i}$. Então, como já vimos no Capítulo 2, Seção 2.9.1 as equações de Newton-Euler podem ser escritas assim:

$$
\begin{gathered}
\overrightarrow{R_{i}}-m_{i} \overrightarrow{A_{i}}=\overrightarrow{0} \\
\overrightarrow{T_{i}}-\left(I_{i} \overrightarrow{\alpha_{i}}+\overrightarrow{w_{i}} \wedge I_{i} \overrightarrow{w_{i}}\right)=\overrightarrow{0}
\end{gathered}
$$

Sendo, 
$m_{i}:$ massa do corpo $P_{i}$

$\vec{A}_{i}$ : aceleração do centro de massa do corpo $P_{i}$

$I_{i}$ : momento de inércia do corpo $P_{i}$ em relação ao seu eixo baricêntrico

$\vec{w}_{i}$ : velocidade angular do centro de massa do corpo $P_{i}$

$\overrightarrow{\alpha_{i}}=\overrightarrow{w_{i}}$ : a aceleração angular do centro de massa do corpo $P_{i}$

Para produzir equações escalares vamos fazer o produto escalar da primeira equação acima por $\frac{\partial \overrightarrow{v_{i}}}{\partial u_{r}}$ e da segunda por $\frac{\partial \overrightarrow{w_{i}}}{\partial u_{r}}$, isto é,

$$
\begin{gathered}
\overrightarrow{R_{i}} \cdot \frac{\partial \overrightarrow{v_{i}}}{\partial u_{r}}-m_{i} \overrightarrow{A_{i}} \cdot \frac{\partial \overrightarrow{v_{i}}}{\partial u_{r}}=0 \\
\overrightarrow{T_{i}} \cdot \frac{\partial \overrightarrow{w_{i}}}{\partial u_{r}}-\left(I_{i} \overrightarrow{\alpha_{i}}+\overrightarrow{w_{i}} \wedge I_{k} \overrightarrow{w_{i}}\right) \cdot \frac{\partial \overrightarrow{w_{i}}}{\partial u_{r}}=0
\end{gathered}
$$

Sendo $\overrightarrow{v_{i}}$ a velocidade linear do centro de gravidade do corpo $P i, u_{r}$ a variável de movimento de Kane (generalized speed) e $r=1,2, \ldots, M$ o seu número.

Somando membro a membro as equações acima e estendendo para $n$ corpos, temos

$$
\sum_{i=1}^{n}\left[\overrightarrow{R_{i}} \cdot \frac{\partial \overrightarrow{v_{i}}}{\partial u_{r}}+\overrightarrow{T_{i}} \cdot \frac{\partial \overrightarrow{w_{i}}}{\partial u_{r}}-\left(m_{i} \overrightarrow{A_{i}} \cdot \frac{\partial \overrightarrow{v_{i}}}{\partial u_{r}}+\left(I_{i} \overrightarrow{\alpha_{i}}+\overrightarrow{w_{i}} \wedge I_{k} \overrightarrow{w_{i}}\right) \cdot \frac{\partial \overrightarrow{w_{i}}}{\partial u_{r}}\right)\right]=0
$$

Então colocando:

$$
F_{r}=\sum_{i=1}^{n}\left(\overrightarrow{R_{i}} \cdot \frac{\partial \overrightarrow{v_{i}}}{\partial u_{r}}+\vec{T}_{i} \cdot \frac{\partial \vec{w}_{i}}{\partial u_{r}}\right)
$$

temos o que se chama de força ativa generalizada do sistema. E

$$
F_{r}^{*}=-\sum_{i=1}^{n}\left(m_{i} \overrightarrow{A_{i}} \cdot \frac{\partial \overrightarrow{v_{i}}}{\partial u_{r}}+\left(I_{i} \overrightarrow{\alpha_{i}}+\vec{w}_{i} \wedge I_{k} \overrightarrow{w_{i}}\right) \cdot \frac{\partial \vec{w}_{i}}{\partial u_{r}}\right)
$$

temos o que se chama de força de inércia generalizada do sistema. De modo que as equações diferenciais dinâmicas de Kane podem ser escritas simplesmente assim:

$$
F_{r}+F_{r}^{*}=0 \quad(r=1,2, \ldots, M)
$$

No caso específico do nosso trabalho, se desenvolvermos as equações do Teorema do Movimento do Baricentro e do Teorema do Momento Angular para o 
corpo $P i$, isto é, $(T M B)_{i}$ e $(T M A)_{i}$ podemos então colocar:

$$
\sum_{i=1}^{n}\left[(T M B)_{i} \cdot \frac{\partial \overrightarrow{v_{i}}}{\partial u_{r}}+(T M A)_{i} \cdot \frac{\partial \overrightarrow{w_{i}}}{\partial u_{r}}\right]=0 \quad(r=1,2, \ldots, M)
$$

\subsection{Aplicação do método de Kane}

Na fig. 8.1 apresentamos o mecanismo esquematicamente desenhado onde mostramos todos os vetores unitários que usaremos para o desenvolvimento da análise, bem como as forças e torques de reação na base. Conforme vimos no Capítulo 6 a base do mecanismo $\mathrm{O}_{2} \mathrm{O}_{6}$ tem comprimento $L_{1}$, a plataforma móvel $E B$ (doravante denominada de elo $J$ ) tem comprimento $2 L_{J}$ e seu centro de gravidade é $J^{*}$. A cadeia ativa direita é composta dos elos $O_{6} A$ ( ou elo $A$ ) e $A B$ (ou elo $B$ ) cujos respectivos comprimentos são $L_{A}$ e $L_{B}$, e os respectivos CG são $A^{*}$ e $B^{*}$. A cadeia ativa esquerda é composta dos elos $O_{2} D$ ( ou elo $D$ ) e $D E$ (ou elo $E$ ) cujos respectivos comprimentos são $L_{D}$ e $L_{E}$, e os respectivos CG são $D^{*}$ e $E^{*}$. A cadeia passiva tem comprimento variável $q_{6}$ porém o elo $H$, parte inferior da cadeia passiva, tem o CG a uma distância $L_{G H}$ do ponto $O_{7}$.

Desprezaremos qualquer efeito das forças de atrito, resistência do ar, deformações e vibrações. Consideraremos todos os corpos como rígidos e situaremos o mecanismo no plano vertical para que o efeito dos pesos próprios dos corpos sejam considerados.

Para o desenvolvimento do método de Kane dividiremos o mecanismo em pares de elos interconectados e utilizaremos então a equação 8.1 para cada par, isto é, o primeiro par será constituído dos elos $A$ e $B$ (cujos índices da variável $u_{r}$ são $r=1$ e $r=2$ ), o segundo par será constituído dos elos D e E (cujos índices da variável $u_{r}$ são $r=3$ e $r=4$ ), e o terceiro par será constituído dos elos $\mathrm{H}$ e $\mathrm{J}$ (cujos índices da variável $u_{r}$ são $r=5$ e $r=6$ ). Vamos assumir que as variáveis $u_{r}$ sejam independentes, de modo que o produto escalar dos vetores TMB e TMA com as derivadas parciais das velocidades em relação a $u_{r}$ dos demais elos, não pertencentes ao par, se anulem. Desta forma, no final, chegaremos a 6 equações que nos possibilitarão calcular 6 incógnitas e através de uma manipulação algébrica podemos chegar a somente 2 equações cujas incógnitas são os torques do atuadores. 


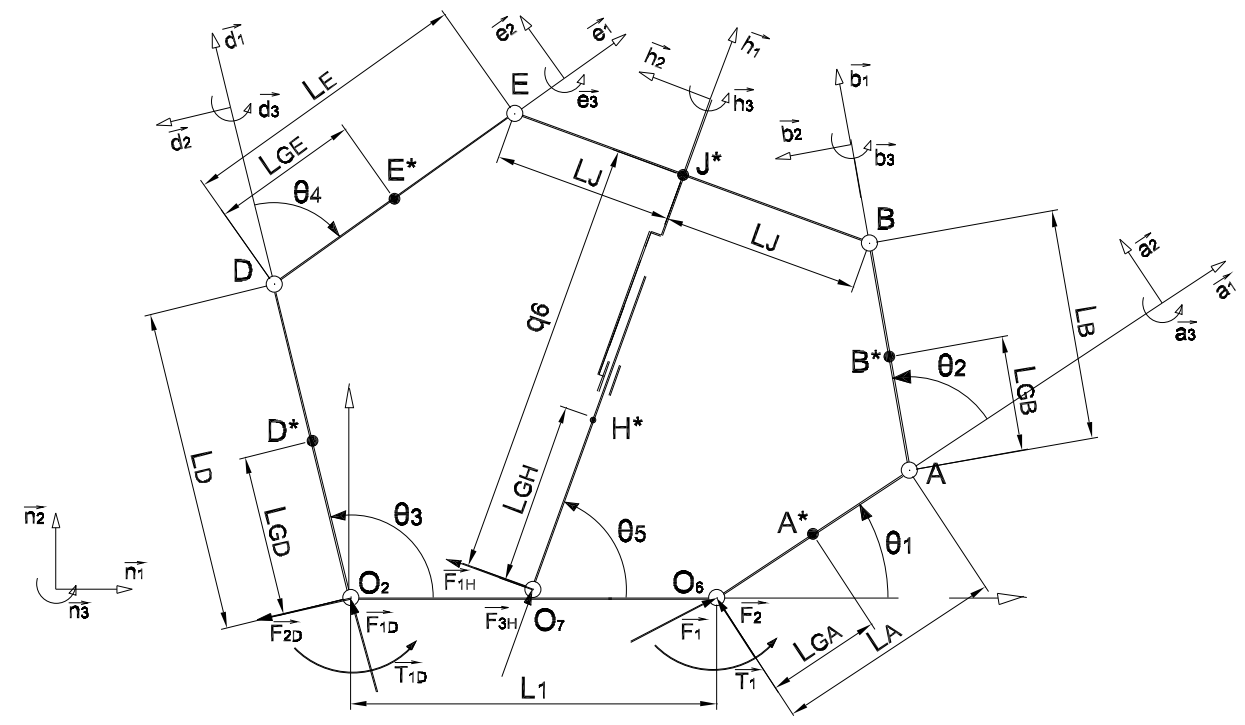

Figura 8.1: Diagrama cinemático do mecanismo

\subsubsection{Especificação das variáveis.}

Consideraremos como variáveis de movimento de Kane (generalized speeds) as derivadas temporais dos ângulos $\theta_{1}, \theta_{2}, \theta_{3}, \theta_{4}, \theta_{5}$, e da distância $q_{6}$, quais sejam: $\dot{\theta}_{1}, \dot{\theta}_{2}, \dot{\theta}_{3}, \dot{\theta}_{4}, \dot{\theta}_{5}$ e $\dot{q}_{6}$, que passaremos também a indicá-las respectivamente por $u_{1}, u_{2}, u_{3}, u_{4}, u_{5}$ e $u_{6}$.

Portanto suas derivadas segundas em relação ao tempo são: $\ddot{\theta}_{1}=\dot{u}_{1}, \ddot{\theta}_{2}=\dot{u}_{2}$, $\ddot{\theta}_{3}=\dot{u}_{3}, \quad \ddot{\theta}_{4}=\dot{u}_{4}, \quad \ddot{\theta}_{5}=\dot{u}_{5} \quad$ e $\ddot{q}_{6}=\dot{u}_{6}$.

Como já dissemos anteriormente, vamos desenvolver o método de Kane admitindo que estas variáveis sejam independentes entre si. A dependência entre elas só vai se manifestar quando a trajetória da garra e a cinemática de posição forem definidas. Ou seja, a dependência entre as variáveis existe, mas na dedução do método de Kane usaremos o artifício da não dependência entre as variáveis.

\subsubsection{Elos A e B}

A fig.8.2 mostra os diagramas de corpo livre dos elos A e B. A partir deles poderemos aplicar o Teorema do Movimento do Baricentro (TMB) e o Teorema do Momento Angular (TMA) para cada elo. 
8.2. Aplicação do métógdo de Kane

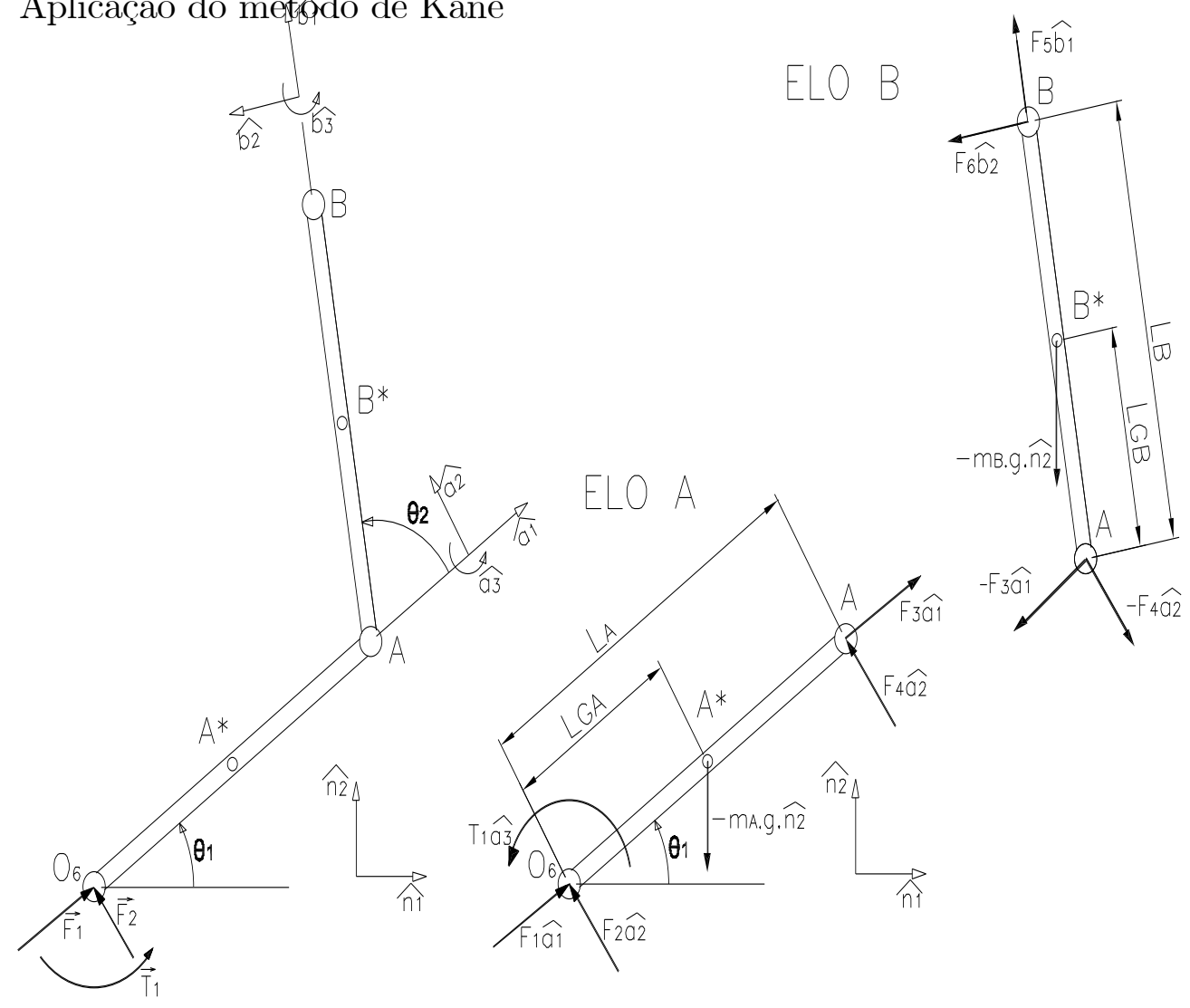

Figura 8.2: Diagramas de corpo livre dos elos A e B

\subsubsection{Elo A}

\section{TMB do elo A.}

Aplicando o Teorema do Movimento do Baricentro ao elo A, considerando as forças atuantes conforme representadas no seu diagrama de corpo livre fig.8.2 chegaremos à seguinte equação:

$$
\left(F_{1}+F_{3}\right) \overrightarrow{a_{1}}+\left(F_{2}+F_{4}\right) \overrightarrow{a_{2}}-m_{A} g \overrightarrow{n_{2}}=m_{A}\left(A_{A}^{n} \overrightarrow{a_{1}}+A_{A}^{t} \overrightarrow{a_{2}}\right)
$$

Onde $g$ é a aceleração da gravidade, $m_{A}$ massa do elo $A ; A_{A}^{n}$ e $A_{A}^{t}$ são as componentes da aceleração do centro de gravidade $A^{*}$ do elo $A$ (os índices inferiores se referem à denominação do respectivo elo), respectivamente normal e tangencial ao seu deslocamento, cuja determinação já vimos no Capítulo 6 .

\section{TMA do elo A}

Aplicando agora o Teorema do Momento Angular ao elo A chegaremos à seguinte equação: 


$$
T_{1} \overrightarrow{a_{3}}+\left(O_{6}-A^{*}\right) \wedge F_{2} \overrightarrow{a_{2}}+\left(A-A^{*}\right) \wedge F_{4} \overrightarrow{a_{2}}=I_{A} \alpha_{A} \overrightarrow{a_{3}}
$$

Onde $I_{A}$ é o momento de inércia do elo $A$ em relação ao seu centro de gravidade e $\alpha_{A}$ é o módulo de sua aceleração angular que calcularemos adiante (os índices se referem à denominação do respectivo elo).

Desenvolvendo chegamos a

$$
\left[T_{1}-L_{G A} F_{2}+\left(L_{A}-L_{G A}\right) F_{4}\right] \overrightarrow{a_{3}}=I_{A} \alpha_{A} \overrightarrow{a_{3}}
$$

\section{Velocidades referentes ao CG do elo A}

A velocidade linear do ponto $A^{*}$ (CG do elo A) é obtida por:

$$
\overrightarrow{V_{A}}=L_{G A} \dot{\theta_{1}} \overrightarrow{a_{2}}=L_{G A} u_{1} \overrightarrow{a_{2}}
$$

A velocidade angular de $A^{*}$ é determinada por:

$$
\overrightarrow{w_{A}}=\dot{\theta}_{1} \overrightarrow{a 3}=u_{1} \overrightarrow{a 3}
$$

A aceleração angular de $A^{*}$ é determinada por:

$$
\overrightarrow{\alpha_{A}}=\ddot{\theta_{1}} \overrightarrow{a 3}=\dot{u_{1}} \overrightarrow{a 3}
$$

\section{Derivadas parciais para $r=1$.}

As derivadas parciais para $r=1$ são as derivadas parciais da velocidade linear e da velocidade angular de $A^{*}$ em relação à variável de índice $r=1$, isto é, variável $u_{1}$. São as seguintes:

$$
\begin{gathered}
\frac{\partial \overrightarrow{V_{A}}}{\partial u_{1}}=L_{G A} \overrightarrow{a_{2}} \\
\frac{\partial \overrightarrow{w_{A}}}{\partial u_{1}}=\overrightarrow{a_{3}}
\end{gathered}
$$

\section{Derivadas parciais para $r=2$}

As derivadas parciais para $r=2$ são as derivadas parciais da velocidade linear e da velocidade angular de $A^{*}$ em relação à variável de índice $r=2$, isto 
é, variável $u_{2}$. São as seguintes:

$$
\begin{aligned}
& \frac{\partial \overrightarrow{V_{A}}}{\partial u_{2}}=0 \\
& \frac{\partial \overrightarrow{w_{A}}}{\partial u_{2}}=0
\end{aligned}
$$

Equações de Kane para o elo $\mathbf{A}$ e $r=1$

\section{Equação (AF1)}

Para construir esta equação, que estamos chamando de (AF1), devemos pegar a equação TMB do elo A, eq.8.2, e multiplicar escalarmente seus dois membros pela derivada parcial $\frac{\partial \overrightarrow{V_{A}}}{\partial u_{1}}$ acima determinada. Doravante vamos simplificar esta descrição adotando a seguinte representação:

$$
T M B_{e l o A} \cdot\left(\frac{\partial \overrightarrow{V_{A}}}{\partial u_{1}}\right)
$$

isto é,

$$
\left[\left(F_{1}+F_{3}\right) \overrightarrow{a_{1}}+\left(F_{2}+F_{4}\right) \overrightarrow{a_{2}}-m_{A} g \overrightarrow{n_{2}}\right] \cdot L_{G A} \overrightarrow{a_{2}}=\left[m_{A}\left(A_{A}^{n} \overrightarrow{a_{1}}+A_{A}^{t} \overrightarrow{a_{2}}\right)\right] \cdot L_{G A} \overrightarrow{a_{2}}
$$

que desenvolvendo chegamos a

$$
F_{2} L_{G A}+F_{4} L_{G A}-m_{A} g L_{G A} c_{1}=m_{A} L_{G A} A_{A}^{t}
$$

\section{Equação (AM1)}

Para construir esta equação, que estamos chamando de (AM1), devemos pegar a equação TMA do elo A, eq.8.3, e multiplicar escalarmente seus dois membros pela derivada parcial $\frac{\partial \overrightarrow{w_{A}}}{\partial u_{1}}$ acima determinada. Doravante vamos simplificar esta descrição adotando a seguinte representação:

$$
T M A_{e l o A} \cdot\left(\frac{\partial \overrightarrow{w_{A}}}{\partial u_{1}}\right)
$$

isto é,

$$
\left[T_{1}-L_{G A} F_{2}+\left(L_{A}-L_{G A}\right) F_{4}\right] \overrightarrow{a_{3}} \cdot \overrightarrow{a_{3}}=I_{A} \alpha_{A} \overrightarrow{a_{3}} \cdot \overrightarrow{a_{3}}
$$


que desenvolvendo chegamos a

$$
T_{1}-L_{G A} F_{2}+L_{A} F_{4}-L_{G A} F_{4}=I_{A} \dot{u}_{1}
$$

\section{Equações de Kane do elo A e $r=2$}

As equações que construiremos agora são similares às anteriores, porém, agora as derivadas parciais serão em relação à variável de índice $r=2$, ou seja, variável $u_{2}$.

1. Equação (AF2)

$$
T M B_{\text {eloA }} \cdot\left(\frac{\partial \overrightarrow{V_{A}}}{\partial u_{2}}\right)=0
$$

Portanto não há equação.

2. Equação (AM2)

$$
T M A_{e l o A} \cdot\left(\frac{\partial \overrightarrow{w_{A}}}{\partial u_{2}}\right)=0
$$

Também não há equação.

\subsubsection{Elo B}

Identicamente ao que fizemos com o elo A vamos fazer para o elo B.

\section{TMB do elo B}

$$
-F_{3} \overrightarrow{a_{1}}-F_{4} \overrightarrow{a_{2}}+F_{5} \overrightarrow{b_{1}}+F_{6} \overrightarrow{b_{2}}-m_{B} g \overrightarrow{n_{2}}=m_{B}\left(A_{B}^{n} \overrightarrow{b_{1}}+A_{B}^{t} \overrightarrow{b_{2}}\right)
$$

\section{TMA do elo B}

$$
\left(A-B^{*}\right) \wedge\left(-F_{3} \overrightarrow{a_{1}}\right)+\left(A-B^{*}\right) \wedge\left(-F_{4} \overrightarrow{a_{2}}\right)+\left(B-B^{*}\right) \wedge\left(F_{6} \overrightarrow{b_{2}}\right)=I_{B} \alpha_{B} \overrightarrow{b_{3}}
$$

Desenvolvendo esta equação chegamos a

$$
\left[-L_{G B} F_{3} s_{2}+L_{G B} F_{4} c_{2}+\left(L_{B}-L_{G B}\right) F_{6}\right] \overrightarrow{b_{3}}=I_{B} \alpha_{B} \overrightarrow{b_{3}}
$$


Velocidades referentes ao CG do elo B

$$
\begin{gathered}
\overrightarrow{V_{B}}=L_{A} \dot{\theta_{1}} \overrightarrow{a_{2}}+L_{G B}\left(\dot{\theta_{1}}+\dot{\theta_{2}}\right) \overrightarrow{b_{2}}=L_{A} u_{1} \overrightarrow{a_{2}}+L_{G B}\left(u_{1}+u_{2}\right) \overrightarrow{b_{2}} \\
\overrightarrow{w_{B}}=\left(\dot{\theta_{1}}+\dot{\theta_{2}}\right) \overrightarrow{a_{3}}=\left(u_{1}+u_{2}\right) \overrightarrow{a_{3}} \\
\overrightarrow{a_{B}}=\left(\ddot{\theta_{1}}+\ddot{\theta_{2}}\right) \overrightarrow{a_{3}}=\left(\dot{u_{1}}+\dot{u_{2}}\right) \overrightarrow{a_{3}}
\end{gathered}
$$

Derivadas parciais para $r=1$.

$$
\begin{gathered}
\frac{\partial \overrightarrow{V_{B}}}{\partial u_{1}}=L_{A} \overrightarrow{a_{2}}+L_{G B} \overrightarrow{b_{2}} \\
\frac{\partial \overrightarrow{w_{B}}}{\partial u_{1}}=\overrightarrow{a_{3}}
\end{gathered}
$$

Derivadas parciais para $r=2$.

$$
\begin{gathered}
\frac{\partial \overrightarrow{V_{B}}}{\partial u_{2}}=L_{G B} \overrightarrow{b_{2}} \\
\frac{\partial \overrightarrow{w_{B}}}{\partial u_{2}}=\overrightarrow{a_{3}}
\end{gathered}
$$

Equações de Kane para o elo B e $r=1$.

1. Equação (BF1)

$$
T M B_{e l o B} \cdot\left(\frac{\partial \overrightarrow{V_{B}}}{\partial u_{1}}\right)
$$

isto é,

$$
\begin{gathered}
\left(-F_{3} \overrightarrow{a_{1}}-F_{4} \overrightarrow{a_{2}}+F_{5} \overrightarrow{b_{1}}+F_{6} \overrightarrow{b_{2}}-m_{B} g \overrightarrow{n_{2}}\right) \cdot\left(L_{A} \overrightarrow{a_{2}}+L_{G B} \overrightarrow{b_{2}}\right) \\
=m_{B}\left(A_{B}^{n} \overrightarrow{b_{1}}+A_{B}^{t} \overrightarrow{b_{2}}\right) \cdot\left(L_{A} \overrightarrow{a_{2}}+L_{G B} \overrightarrow{b_{2}}\right)
\end{gathered}
$$


Desenvolvendo esta equação chegamos a

$$
\begin{gathered}
F_{3} L_{G B} s_{2}-F_{4} L_{A}-F_{4} L_{G B} c_{2}+F_{5} L_{A} s_{2}+F_{6} L_{A} c_{2}+ \\
F_{6} L_{G B}-m_{B} g L_{A} c_{1}-m_{B} g L_{G B} c_{12} \\
=m_{B}\left(A_{B}^{n} L_{A} s_{2}+A_{B}^{t} L_{A} c_{2}+A_{B}^{t} L_{G B}\right)
\end{gathered}
$$

onde $c_{12}=\cos \left(\theta_{1}+\theta_{2}\right)$.

2. Equação (BM1)

$$
T M A_{e l o B} \cdot\left(\frac{\partial \overrightarrow{w_{B}}}{\partial u_{1}}\right)
$$

isto é,

$$
\left[-L_{G B} F_{3} s_{2}+L_{G B} F_{4} c_{2}+\left(L_{B}-L_{G B}\right) F_{6}\right] \overrightarrow{b_{3}} \cdot \overrightarrow{a_{3}}=I_{B} \alpha_{B} \overrightarrow{b_{3}} \cdot \overrightarrow{a_{3}}
$$

que desenvolvendo chegamos a

$$
-L_{G B} F_{3} s_{2}+L_{G B} F_{4} c_{2}+\left(L_{B}-L_{G B}\right) F_{6}=I_{B}\left(\dot{u_{1}}+\dot{u_{2}}\right)
$$

Equações de Kane para o elo B e $r=2$.

1. Equação (BF2)

$$
T M B_{e l o B} \cdot\left(\frac{\partial \overrightarrow{V_{B}}}{\partial u_{2}}\right)
$$

isto é,

$$
\begin{gathered}
\left(-F_{3} \overrightarrow{a_{1}}-F_{4} \overrightarrow{a_{2}}+F_{5} \overrightarrow{b_{1}}+F_{6} \overrightarrow{b_{2}}-m_{B} g \overrightarrow{n_{2}}\right) \cdot L_{G B} \overrightarrow{b_{2}} \\
=\left[m_{B}\left(A_{B}^{n} \overrightarrow{b_{1}}+A_{B}^{t} \overrightarrow{b_{2}}\right) \cdot L_{G B} \overrightarrow{b_{2}}\right]
\end{gathered}
$$

que desenvolvendo chegamos a

$$
L_{G B} F_{3} s_{2}-L_{G B} F_{4} c_{2}+L_{G B} F_{6}-m_{B} g L_{G B} c_{12}=m_{B} L_{G B} A_{B}^{t}
$$


2. Equação (BM2)

$$
T M A_{\text {eloB }} \cdot\left(\frac{\partial \overrightarrow{w_{B}}}{\partial u_{2}}\right)
$$

isto é,

$$
\left[-L_{G B} F_{3} s_{2}+L_{G B} F_{4} c_{2}+\left(L_{B}-L_{G B}\right) F_{6}\right] \overrightarrow{b_{3}} \overrightarrow{a_{3}}=I_{B} \alpha_{B} \overrightarrow{b_{3}} \cdot \overrightarrow{a_{3}}
$$

que desenvolvendo chegamos a

$$
-L_{G B} F_{3} s_{2}+L_{G B} F_{4} c_{2}+\left(L_{B}-L_{G B}\right) F_{6}=I_{B}\left(\dot{u_{1}}+\dot{u_{2}}\right)
$$

\subsubsection{Resultado para os elos A e B}

Agora utilizando a fórmula 8.1 podemos montar as equações de Kane do par de elos A e B para $r=1$, que vamos chamar de equação (AB1) e $r=2$, que chamaremos de equação (AB2).

\section{Equação $(\mathrm{AB1})=(\mathrm{AF} 1)+(\mathrm{AM} 1)+(\mathrm{BF} 1)+(\mathrm{BM} 1)$}

A equação (AB1) é obtida somando membro a membro as equações 8.4, 8.5, 8.8 e 8.9. O resultado após as simplificações é o seguinte:

$$
\begin{aligned}
& T_{1}+F_{5} L_{A} s_{2}+F_{6} L_{A} c_{2}+F_{6} L_{B}-m_{A} g L_{G A} c_{1}-m_{B} g L_{A} c_{1}-m_{B} g L_{G B} c_{12} \\
& =\left(I_{A}+I_{B}\right) \dot{u_{1}}+I_{B} \dot{u_{2}}+m_{A} L_{G A} A_{A}^{t}+m_{B}\left(A_{B}^{n} L_{A} s_{2}+A_{B}^{t} L_{A} c_{2}+A_{B}^{t} L_{G B}\right)
\end{aligned}
$$

\section{Equação $(\mathrm{AB2})=(\mathrm{AF} 2)+(\mathrm{AM} 2)+(\mathrm{BF} 2)+(\mathrm{BM} 2)$}

A equação (AB2) é obtida somando membro a membro as equações 8.10 e 8.11. O resultado após as simplificações é o seguinte:

$$
F_{6} L_{B}-m_{B} g L_{G B} c_{12}=m_{B} L_{G B} A_{B}^{t}+I_{B}\left(\dot{u_{1}}+\dot{u_{2}}\right)
$$

\subsubsection{Elos D e E}

Tudo que fizemos para o par A e B vamos semelhantemente fazer para o par D e E. 


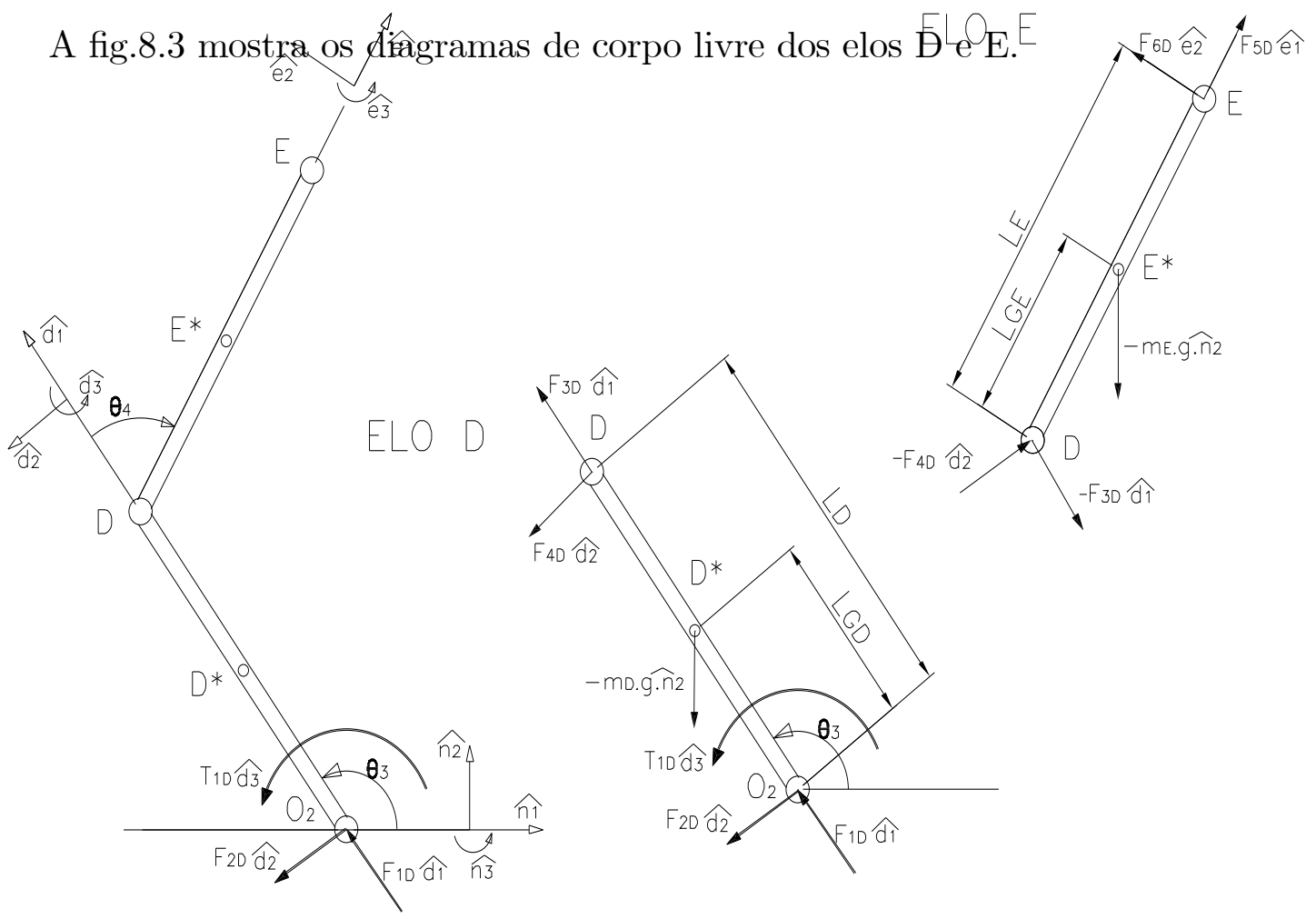

Figura 8.3: Diagramas de corpo livre dos elos D e E

\subsubsection{Elo D}

\section{TMB do elo D}

$$
\left(F_{1}^{D}+F_{3}^{D}\right) \overrightarrow{d_{1}}+\left(F_{2}^{D}+F_{4}^{D}\right) \overrightarrow{d_{2}}-m_{D} g \overrightarrow{n_{2}}=m_{D}\left(A_{D}^{n} \overrightarrow{d_{1}}+A_{D}^{t} \overrightarrow{d_{2}}\right)
$$

TMA do elo D

$$
\left[T_{1}^{D}-L_{G D} F_{2}^{D}+\left(L_{D}-L_{G D}\right) F_{4}^{D}\right] \overrightarrow{d_{3}}=I_{D} \alpha_{D} \overrightarrow{d_{3}}
$$

\section{Velocidades referentes ao CG do elo D}

$$
\begin{gathered}
\overrightarrow{V_{D^{*}}}=L_{G D} \dot{\theta}_{3} \overrightarrow{d_{2}}=L_{G D} \overrightarrow{u_{3}} \overrightarrow{d_{2}} \\
\overrightarrow{w_{D^{*}}}=\dot{\theta}_{3} \overrightarrow{d_{3}}=u_{3} \overrightarrow{d_{3}} \\
\overrightarrow{\alpha_{D^{*}}}=\ddot{\theta}_{3} \overrightarrow{d_{3}}=\dot{u}_{3} \overrightarrow{d_{3}}
\end{gathered}
$$


Derivadas parciais para $r=3$

$$
\begin{gathered}
\frac{\partial \overrightarrow{V_{D}}}{\partial u_{3}}=L_{G D} \overrightarrow{d_{2}} \\
\frac{\partial \overrightarrow{w_{D}}}{\partial u_{3}}=\overrightarrow{d_{3}}
\end{gathered}
$$

Derivadas parciais para $r=4$

$$
\begin{aligned}
& \frac{\partial \overrightarrow{V_{D}}}{\partial u_{4}}=0 \\
& \frac{\partial \overrightarrow{w_{D}}}{\partial u_{4}}=0
\end{aligned}
$$

Equações de Kane para o elo D e $r=3$

1. Equação (DF3)

$$
T M B_{e l o D} \cdot\left(\frac{\partial \overrightarrow{V_{D}}}{\partial u_{3}}\right)
$$

isto é,

$$
F_{2}^{D} L_{G D}+F_{4}^{D} L_{G D}-m_{D} g L_{G D} c_{3}=m_{D} L_{G D} A_{D}^{t}
$$

2. Equação (DM3)

$$
T M A_{\text {eloD }} \cdot\left(\frac{\partial \overrightarrow{w_{D}}}{\partial u_{3}}\right)
$$

isto é,

$$
T_{1}^{D}-L_{G D} F_{2}^{D}+\left(L_{D}-L_{G D}\right) F_{4}^{D}=I_{D} \dot{u}_{3}
$$

Equações de Kane do elo D e $r=4$

1. Equação (DF4)

$$
T M B_{\text {eloD }} \cdot\left(\frac{\partial \overrightarrow{V_{D}}}{\partial u_{4}}\right)=0
$$


Portanto não há equação.

2. Equação (DM4)

$$
T M A_{\text {eloD }} \cdot\left(\frac{\partial \overrightarrow{w_{D}}}{\partial u_{4}}\right)=0
$$

Também não há equação.

\subsubsection{Elo E}

\section{TMB do elo $\mathrm{E}$}

$$
-F_{3}^{D} \overrightarrow{d_{1}}-F_{4}^{D} \overrightarrow{d_{2}}+F_{5}^{D} \overrightarrow{e_{1}}+F_{6}^{D} \overrightarrow{e_{2}}-m_{E} g \overrightarrow{n_{2}}=m_{E}\left(A_{E}^{n} \overrightarrow{e_{1}}+A_{E}^{t} \overrightarrow{e_{2}}\right)
$$

\section{TMA do elo E}

$$
\left[-L_{G E} F_{3}^{D} s_{4}+L_{G E} F_{4}^{D} c_{4}+\left(L_{E}-L_{G E}\right) F_{6}^{D}\right] \overrightarrow{e_{3}}=I_{E} \alpha_{E} \overrightarrow{e_{3}}
$$

Velocidades referentes ao CG do elo E

$$
\begin{aligned}
& \overrightarrow{V_{E^{*}}}=L_{D} \dot{\theta_{3}} \overrightarrow{d_{2}}+L_{G E}\left(\dot{\theta_{3}}+\dot{\theta_{4}}\right) \overrightarrow{e_{2}}=L_{D} u_{3} \overrightarrow{d_{2}}+L_{G E}\left(u_{3}+u_{4}\right) \overrightarrow{e_{2}} \\
& \overrightarrow{w_{E^{*}}}=\left(\dot{\theta_{3}}+\dot{\theta_{4}}\right) \overrightarrow{d_{3}}=\left(u_{3}+u_{4}\right) \overrightarrow{d_{3}} \\
& \overrightarrow{\alpha_{E^{*}}}=\left(\ddot{\theta_{3}}+\ddot{\theta_{4}}\right) \overrightarrow{d_{3}}=\left(\dot{u_{3}}+\dot{u_{4}}\right) \overrightarrow{d_{3}}
\end{aligned}
$$

Derivadas parciais para $r=3$.

$$
\begin{gathered}
\frac{\partial \overrightarrow{V_{E}}}{\partial u_{3}}=L_{D} \overrightarrow{d_{2}}+L_{G E} \overrightarrow{e_{2}} \\
\frac{\partial \overrightarrow{w_{E}}}{\partial u_{3}}=\overrightarrow{d_{3}}
\end{gathered}
$$

Derivadas parciais para $r=4$.

$$
\frac{\partial \overrightarrow{V_{E}}}{\partial u_{4}}=L_{G E} \overrightarrow{e_{2}}
$$




$$
\frac{\partial \overrightarrow{w_{E}}}{\partial u_{4}}=\overrightarrow{d_{3}}
$$

Equações de Kane para o elo E e $r=3$.

1. Equação (EF3)

$$
T M B_{e l o E} \cdot\left(\frac{\partial \overrightarrow{V_{E}}}{\partial u_{3}}\right)
$$

isto é,

$$
\begin{gathered}
F_{3}^{D} L_{G E} s_{4}-F_{4}^{D} L_{D}-F_{4}^{D} L_{G E} c_{4}+F_{5}^{D} L_{D} s_{4}+F_{6}^{D} L_{D} c_{4}+ \\
F_{6}^{D} L_{G E}-m_{E} g L_{D} c_{3}-m_{E} g L_{G E} c_{34} \\
=m_{E}\left(A_{E}^{n} L_{D} s_{4}+A_{E}^{t} L_{D} c_{4}+A_{E}^{t} L_{G E}\right)
\end{gathered}
$$

onde $c_{34}=\cos \left(\theta_{3}+\theta_{4}\right)$.

2. Equação (EM3)

$$
T M A_{e l o E} \cdot\left(\frac{\partial \overrightarrow{w_{E}}}{\partial u_{3}}\right)
$$

isto é,

$$
-L_{G E} F_{3}^{D} s_{4}+L_{G E} F_{4}^{D} c_{4}+\left(L_{E}-L_{G E}\right) F_{6}^{D}=I_{E}\left(\dot{u_{3}}+\dot{u} 4\right)
$$

Equações de Kane para o elo $\mathbf{E}$ e $r=4$.

1. Equação (EF4)

$$
T M B_{\text {eloE }} \cdot\left(\frac{\partial \overrightarrow{V_{E}}}{\partial u_{4}}\right)
$$

isto é,

$$
L_{G E} F_{3}^{D} s_{4}-L_{G E} F_{4}^{D} c_{4}+L_{G E} F_{6}^{D}-m_{E} g L_{G E} c_{34}=m_{E} L_{G E} A_{E}^{t}
$$


2. Equação (EM4)

$$
T M A_{\text {eloE }} \cdot\left(\frac{\partial \overrightarrow{w_{E}}}{\partial u_{4}}\right)
$$

isto é,

$$
-L_{G E} F_{3}^{D} s_{4}+L_{G E} F_{4}^{D} c_{4}+\left(L_{E}-L_{G E}\right) F_{6}^{D}=I_{E}\left(\dot{u_{3}}+\dot{u_{4}}\right)
$$

\subsubsection{Resultado para os elos D e E}

\section{Equação $($ DE3 $)=($ DF3 $)+($ DM3 $)+($ EF3 $)+($ EM3 $)$}

A equação (DE3) é obtida somando membro a membro as equações $8.16,8.17,8.20$ e 8.21. O resultado após as simplificações é o seguinte:

$$
\begin{aligned}
& T_{1}^{D}+F_{5}^{D} L_{D} s_{4}+F_{6}^{D} L_{D} c_{4}+F_{6}^{D} L_{E}-m_{D} g L_{G D} c_{3}-m_{E} g L_{D} c_{3}-m_{E} g L_{G E} c_{34} \\
& =\left(I_{D}+I_{E}\right) \dot{u_{3}}+I_{E} \dot{u}_{4}+m_{D} L_{G D} A_{D}^{t}+m_{E}\left(A_{E}^{n} L_{D} s_{4}+A_{E}^{t} L_{D} c_{4}+A_{E}^{t} L_{G E}\right)
\end{aligned}
$$

\section{Equação $(\mathrm{DE} 4)=(\mathrm{DF} 4)+(\mathrm{DM} 4)+(\mathrm{EF} 4)+(\mathrm{EM} 4)$}

A equação (DE4) é obtida somando membro a membro as equações 8.22 e 8.23. O resultado após as simplificações é o seguinte:

$$
F_{6}^{D} L_{E}-m_{E} g L_{G E} c_{34}=m_{E} L_{G E} A_{E}^{t}+I_{E}\left(\dot{u_{3}}+\dot{u_{4}}\right)
$$

\subsubsection{Elos $\mathbf{H}$ e J}

A fig.8.4 mostra os diagramas de corpo livre dos elos H e J. Podemos então desenvolver o TMB e TMA de cada elo. Entretanto é importante observarmos que as variáveis de configuração neste caso são o ângulo $\theta_{5}$ e o comprimento $q_{6}$. As variáveis de movimento de Kane são $u_{5}=\theta_{5}$ e $u_{6}=\dot{q}_{6}$. 


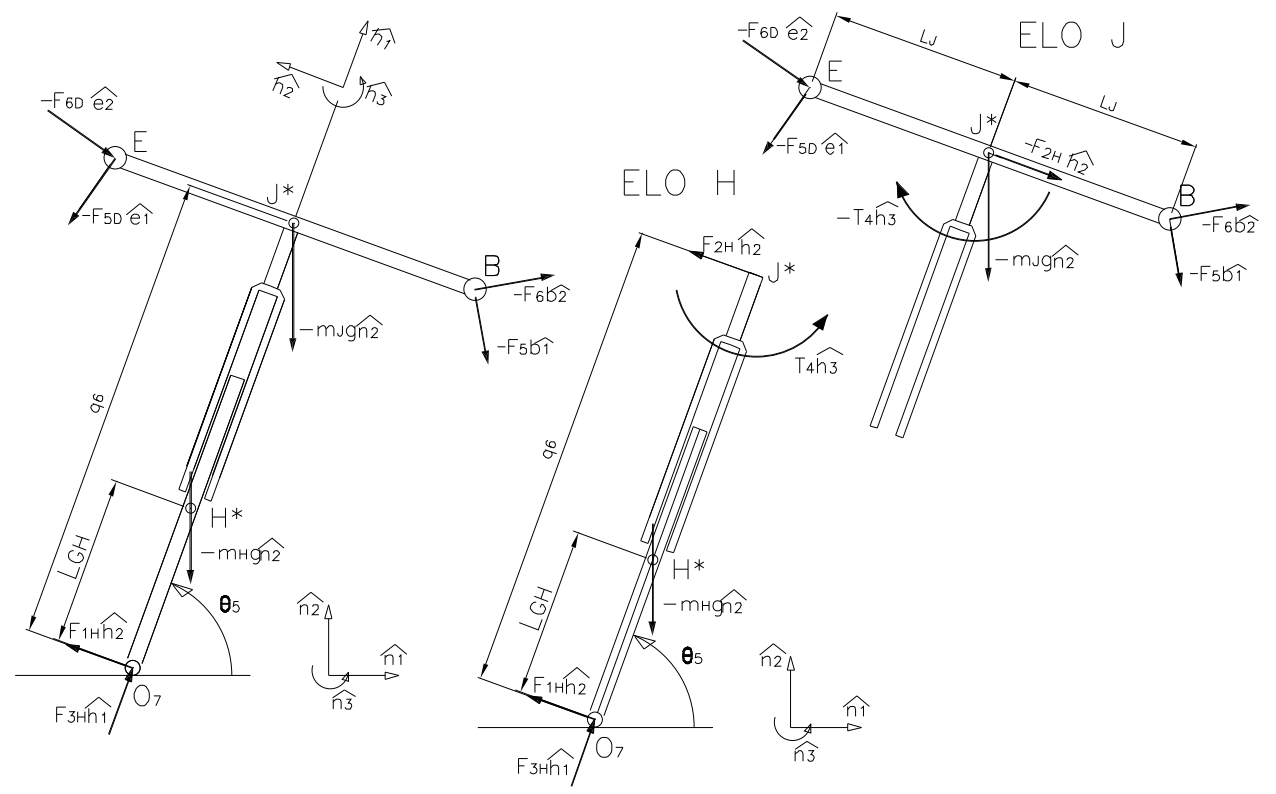

Figura 8.4: Diagramas de corpo livre dos elos $\mathrm{H}$ e J

\subsubsection{Elo $\mathrm{H}$}

\section{TMB do elo $\mathbf{H}$.}

$$
F_{1}^{H} \overrightarrow{h_{2}}+F_{2}^{H} \overrightarrow{h_{2}}+F_{3}^{H} \overrightarrow{h_{1}}-m_{H} g \overrightarrow{n_{2}}=m_{H}\left(A_{H}^{n} \overrightarrow{h_{1}}+A_{H}^{t} \overrightarrow{h_{2}}\right)
$$

\section{TMA do elo $\mathbf{H}$}

$$
T_{4} \overrightarrow{h_{3}}+\left(O_{7}-H^{*}\right) \wedge F_{1}^{H} \overrightarrow{h_{2}}+\left(J^{*}-H^{*}\right) \wedge F_{2}^{H} \overrightarrow{h_{2}}=I_{H} \alpha_{h} \overrightarrow{h_{3}}
$$

Desenvolvendo esta equação chegamos a:

$$
\left[T_{4}-L_{G H} F_{1}^{H}+\left(q_{6}-L_{G H}\right) F_{2}^{H}\right] \overrightarrow{h_{3}}=I_{H} \alpha_{h} \overrightarrow{h_{3}}
$$

Velocidades referentes ao CG do elo $\mathbf{H}$

$$
\begin{gathered}
\overrightarrow{V_{H^{*}}}=L_{G H} \dot{\theta_{5}} \overrightarrow{h_{2}}=L_{G H} u_{5} \overrightarrow{h_{2}} \\
\overrightarrow{w_{H^{*}}}=\dot{\theta_{5}} \overrightarrow{h_{3}}=u_{5} \overrightarrow{h_{3}} \\
\overrightarrow{\alpha_{H^{*}}}=\ddot{\theta_{5}} \overrightarrow{h_{3}}=\dot{u_{5}} \overrightarrow{h_{3}}
\end{gathered}
$$


Derivadas parciais para $r=5$.

$$
\begin{gathered}
\frac{\partial \overrightarrow{V_{H}}}{\partial u_{5}}=L_{G H} \overrightarrow{h_{2}} \\
\frac{\partial \overrightarrow{w_{H}}}{\partial u_{5}}=\overrightarrow{h_{3}}
\end{gathered}
$$

Derivadas parciais para $r=6$.

$$
\begin{aligned}
& \frac{\partial \overrightarrow{V_{H}}}{\partial u_{6}}=0 \\
& \frac{\partial \overrightarrow{w_{H}}}{\partial u_{6}}=0
\end{aligned}
$$

Equações de Kane do elo $\mathbf{H}$ e $r=5$.

1. Equação (HF5)

$$
T M B_{e l o H} \cdot\left(\frac{\partial \overrightarrow{V_{H}}}{\partial u_{5}}\right)
$$

isto é,

$$
\left(F_{1}^{H} \overrightarrow{h_{2}}+F_{2}^{H} \overrightarrow{h_{2}}+F_{3}^{H} \overrightarrow{h_{1}}-m_{H} g \overrightarrow{n_{2}}\right) \cdot L_{G H} \overrightarrow{h_{2}}=\left[m_{H}\left(A_{H}^{n} \overrightarrow{h_{1}}+A_{H}^{t} \overrightarrow{h_{2}}\right)\right] \cdot L_{G H} \overrightarrow{h_{2}}
$$

Desenvolvendo esta equação chegamos a

$$
L_{G H} F_{1}^{H}+L_{G H} F_{2}^{H}-L_{G H} m_{H} g c_{5}=L_{G H} m_{H} A_{H}^{t}
$$

2. Equação (HM5)

$$
T M A_{\text {eloH }} \cdot\left(\frac{\partial \overrightarrow{w_{H}}}{\partial u_{5}}\right)
$$

isto é,

$$
\left[\left[T_{4}-L_{G H} F_{1}^{H}+\left(q_{6}-L_{G H}\right) F_{2}^{H}\right] \overrightarrow{h_{3}}\right] \cdot \overrightarrow{h_{3}}=\left(\begin{array}{ll}
I_{H} & \dot{u_{5}} \overrightarrow{h_{3}}
\end{array}\right) \cdot \overrightarrow{h_{3}}
$$


Desenvolvendo esta equação chegamos a

$$
T_{4}-L_{G H} F_{1}^{H}+\left(q_{6}-L_{G H}\right) F_{2}^{H}=I_{H} \dot{u}_{5}
$$

Equações de Kane do corpo $\mathbf{H}$ e $r=6$

1. Equação (HF6)

$$
T M B_{\text {eloH }} \cdot\left(\frac{\partial \overrightarrow{V_{H}}}{\partial u_{6}}\right)=0
$$

isto é, não existe equação.

1. Equação (HM6)

$$
T M A_{\text {eloH }} \cdot\left(\frac{\partial \overrightarrow{w_{H}}}{\partial u_{6}}\right)=0
$$

Portanto também não existe equação.

\subsubsection{Elo J}

\section{TMB do elo J}

$$
-F_{6}^{D} \overrightarrow{e_{2}}-F_{5}^{D} \overrightarrow{e_{1}}-F_{2}^{H} \overrightarrow{h_{2}}-F_{5} \overrightarrow{b_{1}}-F_{6} \overrightarrow{b_{2}}-m_{J} g \overrightarrow{n_{2}}=m_{J}\left(A_{J}^{n} \overrightarrow{h_{2}}+A_{J}^{t} \overrightarrow{h_{1}}\right)
$$

\section{TMA do elo J}

$$
\begin{gathered}
\left(E-J^{*}\right) \wedge\left(-F_{6}^{D} \overrightarrow{e_{2}}\right)+\left(E-J^{*}\right) \wedge\left(-F_{5}^{D} \overrightarrow{e_{1}}\right)+\left(B-J^{*}\right) \wedge\left(-F_{5} \overrightarrow{b_{1}}\right)+ \\
\left(B-J^{*}\right) \wedge\left(-F_{6} \overrightarrow{b_{2}}\right)-T_{4} \overrightarrow{h_{3}}=I_{J} \alpha_{J} \overrightarrow{h_{3}}
\end{gathered}
$$

Desenvolvendo esta equação chegamos a

$$
\left(-T_{4}+L_{J} F_{6}^{D} s_{\lambda}+L_{J} F_{5}^{D} c_{\lambda}-L_{J} F_{5} c_{\alpha}+L_{J} F_{6} s_{\alpha}\right) \overrightarrow{h_{3}}=I_{J} \alpha_{J} \overrightarrow{h_{3}}
$$

onde

$$
\begin{array}{ll}
s_{\lambda}=\operatorname{sen}\left(\theta_{5}-\theta_{4}-\theta_{3}\right) & c_{\lambda}=\cos \left(\theta_{5}-\theta_{4}-\theta_{3}\right) \\
s_{\alpha}=\operatorname{sen}\left(\theta_{1}+\theta_{2}-\theta_{5}\right) & c_{\alpha}=\cos \left(\theta_{1}+\theta_{2}-\theta_{5}\right)
\end{array}
$$




\section{Velocidades referentes ao CG do elo J}

$$
\begin{gathered}
\overrightarrow{V_{J^{*}}}=\dot{q_{6}} \overrightarrow{h_{1}}+q_{6} \dot{\theta_{5}} \overrightarrow{h_{2}}=u_{6} \overrightarrow{h_{1}}+q_{6} u_{5} \overrightarrow{h_{2}} \\
\overrightarrow{w_{J^{*}}}=\dot{\theta_{5}} \overrightarrow{h_{3}}=u_{5} \overrightarrow{h_{3}} \\
\overrightarrow{\alpha_{J^{*}}}=\ddot{\theta_{5}} \overrightarrow{h_{3}}=\dot{u_{5}} \overrightarrow{h_{3}}
\end{gathered}
$$

Derivadas parciais para $r=5$.

$$
\begin{gathered}
\frac{\partial \overrightarrow{V_{J}}}{\partial u_{5}}=q_{6} \overrightarrow{h_{2}} \\
\frac{\partial \overrightarrow{w_{J}}}{\partial u_{5}}=\overrightarrow{h_{3}}
\end{gathered}
$$

Derivadas parciais para $r=6$.

$$
\begin{gathered}
\frac{\partial \overrightarrow{V_{J}}}{\partial u_{6}}=\overrightarrow{h_{1}} \\
\frac{\partial \overrightarrow{w_{J}}}{\partial u_{6}}=0
\end{gathered}
$$

Equações de Kane do elo J e $r=5$.

1. Equação (JF5)

$$
T M B_{\text {eloJ }} \cdot\left(\frac{\partial \overrightarrow{V_{J}}}{\partial u_{5}}\right)
$$

isto é,

$$
\begin{gathered}
\left(-F_{6}^{D} \overrightarrow{e_{2}}-F_{5}^{D} \overrightarrow{e_{1}}-F_{2}^{H} \overrightarrow{h_{2}}-F_{5} \overrightarrow{b_{1}}-F_{6} \overrightarrow{b_{2}}-m_{J} g \overrightarrow{n_{2}}\right) \cdot q_{6} \overrightarrow{h_{2}} \\
=\left[m_{J}\left(A_{J}^{n} \overrightarrow{h_{2}}+A_{J}^{t} \overrightarrow{h_{1}}\right)\right] \cdot q_{6} \overrightarrow{h_{2}}
\end{gathered}
$$

Desenvolvendo esta equação chegamos a

$$
-F_{6}^{D} q_{6} c_{\lambda}+F_{5}^{D} q_{6} s_{\lambda}-F_{2}^{H} q_{6}-F_{5} q_{6} s_{\alpha}-F_{6} q_{6} c_{\alpha}-m_{J} g q_{6} c_{5}=m_{J} q_{6} A_{J}^{n}
$$


2. Equação (JM5)

$$
T M A_{\text {eloJ }} \cdot\left(\frac{\partial \overrightarrow{w_{J}}}{\partial u_{5}}\right)
$$

isto é,

$$
-T_{4}+L_{J} F_{6}^{D} s_{\lambda}+L_{J} F_{5}^{D} c_{\lambda}-L_{J} F_{5} c_{\alpha}+L_{J} F_{6} s_{\alpha}=I_{J} \dot{u_{5}}
$$

Equações de Kane do elo J e $r=6$.

1. Equação (JF6)

$$
T M B_{\text {elo }} \cdot\left(\frac{\partial \overrightarrow{V_{J}}}{\partial u_{6}}\right)
$$

isto é,

$$
\begin{gathered}
\left(-F_{6}^{D} \overrightarrow{e_{2}}-F_{5}^{D} \overrightarrow{e_{1}}-F_{2}^{H} \overrightarrow{h_{2}}-F_{5} \overrightarrow{b_{1}}-F_{6} \overrightarrow{b_{2}}-m_{J} g \overrightarrow{n_{2}}\right) \cdot \overrightarrow{h_{1}} \\
=\left[m_{J}\left(A_{J}^{n} \overrightarrow{h_{2}}+A_{J}^{t} \overrightarrow{h_{1}}\right)\right] \cdot \overrightarrow{h_{1}}
\end{gathered}
$$

Desenvolvendo chegamos a

$$
-F_{6}^{D} s_{\lambda}-F_{5}^{D} c_{\lambda}-F_{5} c_{\alpha}+F_{6} s_{\alpha}-m_{J} g s_{5}=m_{J} A_{J}^{t}
$$

2. Equação (JM6)

$$
T M A_{\text {eloJ }} \cdot\left(\frac{\partial \overrightarrow{w_{J}}}{\partial u_{6}}\right)=0
$$

Portanto não há equação.

\subsubsection{Resultado para os elos $\mathrm{H}$ e J}

\section{Equação $($ HJ5 $)=($ HF5 $)+($ HM5 $)+($ JF5 $)+($ JM5 $)$}

A equação (HJ5) é obtida somando membro a membro as equações 8.28, 8.29, 8.32 e 8.33. O resultado após as simplificações é o seguinte:

$$
\begin{gathered}
-F_{5}\left(L_{j} c_{\alpha}+q_{6} s_{\alpha}\right)+F_{5}^{D}\left(L_{j} c_{\lambda}+q_{6} s_{\lambda}\right)+F_{6}\left(L_{j} s_{\alpha}-q_{6} c_{\alpha}\right)+ \\
F_{6}^{D}\left(L_{j} s_{\lambda}-q_{6} c_{\lambda}\right)-L_{G H} m_{H} g c_{5}-m_{J} g q_{6} c_{5}
\end{gathered}
$$




$$
=L_{G H} m_{H} A_{H}^{t}+m_{J} q_{6} A_{J}^{n}+\left(I_{H}+I_{J}\right) \dot{u}_{5}
$$

2. Equação $($ HJ6 $)=($ HF6 $)+($ HM6 $)+(J F 6)+(J M 6)$

A equação (HJ6) será, portanto, a equação 8.34, isto é,

$$
-F_{6}^{D} s_{\lambda}-F_{5}^{D} c_{\lambda}-F_{5} c_{\alpha}+F_{6} s_{\alpha}-m_{J} g s_{5}=m_{J} A_{J}^{t}
$$

\subsubsection{Resultado geral}

Com isto completamos as 6 equações que o método de Kane nos fornece que são as equações (AB1), (AB2), (DE3), (DE4), (HJ5) e (HJ6) que estão respectivamente numeradas por $8.12,8.13,8.24,8.25,8.35$ e 8.36 .

\subsection{Determinação dos torques e forças}

\subsubsection{Torques de reação $T_{1} \mathbf{e} T_{1}^{D}$}

Os torques de reação dos atuadores, $T_{1}$ e $T_{1}^{D}$, e as forças $F_{5}, F_{5}^{D}, F_{6}$ e $F_{6}^{D}$ que atuam nas juntas da plataforma móvel, são exatamente as seis incógnitas das seis equações 8.12, 8.13, 8.24, 8.25, 8.35 e 8.36 que deduzimos na seção 8.2.

No Anexo J apresentamos o programa Mathematica que deduz as equações de cada uma destas incógnitas.

A equação do momento de reação no atuador $O_{2}$ é:

$$
\begin{gathered}
T_{1}^{D}=m_{D} L_{G D}\left(A_{D}^{t}+g c_{3}\right)+m_{E} L_{D}\left(A_{E}^{t} c_{4}+A_{E}^{n} s_{4}+g c_{3}\right)+m_{E} L_{G E}\left(A_{E}^{t}+g c_{34}\right)+ \\
I_{D} \dot{u}_{3}+I_{E} \dot{u}_{3}+I_{E} \dot{u}_{4}+\frac{\left(L_{D} c_{4}+L_{E}\right)\left(-m_{E} L_{G E} A_{E}^{t}-m_{E} L_{G E} g c_{34}-I_{E} \dot{u}_{3}-I_{E} \dot{u}_{4}\right)}{L_{E}}+ \\
\frac{\left(-2 L_{J} c_{\alpha} s_{\lambda}+q_{6} c_{\alpha \lambda}\right)\left(m_{E} L_{G E} A_{E}^{t}+m_{E} L_{G E} g c_{34}+I_{E} \dot{u}_{3}+I_{E} \dot{u}_{4}\right)}{L_{D}\left(-2 L_{J} c_{\alpha} c_{\lambda}-q_{6} s_{\alpha \lambda}\right)}+ \\
\frac{\left(-m_{\alpha} L_{J}-q_{6} s_{\alpha}\right)\left(m_{J} A_{J}^{t}+m_{J} g s_{5}\right)}{-2 L_{J} c_{\alpha} c_{\lambda}-q_{6} s_{\alpha \lambda}}+ \\
\left.\frac{L_{\alpha}\left(m_{H} A_{H}^{t} L_{G H}+m_{J} A_{J}^{n} q_{6}+m_{H} L_{G H} g c_{5}+m_{J} q_{6} g c_{5}+I_{H} \dot{u}_{5}+I_{J} \dot{u}_{5}\right)}{-2 L_{J} c_{\alpha} c_{\lambda}-q_{6} s_{\alpha \lambda}}\right\}
\end{gathered}
$$

A equação do momento de reação no atuador $O_{6}$ é: 


$$
\begin{aligned}
& T_{1}= m_{A} L_{G A}\left(A_{A}^{t}+g c_{1}\right)+m_{B} L_{A}\left(A_{B}^{t} c_{2}+A_{B}^{n} s_{2}+g c_{1}\right)+ \\
& m_{B} L_{G B}\left(A_{B}^{t}+g c_{12}\right)+I_{A} \dot{u}_{1}+I_{B} \dot{u}_{1}+I_{B} \dot{u}_{2}+ \\
& \frac{\left(L_{A} c_{2}+L_{B}\right)\left(-m_{B} L_{G B} A_{B}^{t}-m_{B} L_{G B} g c_{12}-I_{B} \dot{u}_{1}-I_{B} \dot{u}_{2}\right)}{L_{B}}+
\end{aligned}
$$

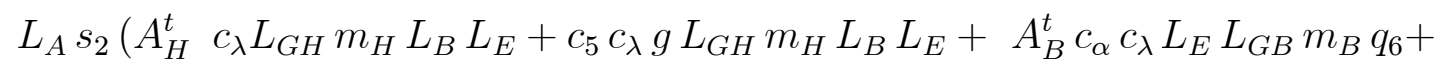
$c_{12} c_{\alpha} c_{\lambda} g L_{E} L_{G B} m_{B} q_{6}+A_{E}^{t} c_{\lambda}^{2} L_{B} L_{G E} m_{E} q_{6}+c_{34} c_{\lambda}^{2} g L_{B} L_{G E} m_{E} q_{6}+$ $A_{J}^{n} c_{\lambda} L_{B} L_{E} m_{J} q_{6}+c_{5} c_{\lambda} g m_{J} q_{6} L_{B} L_{E}+c_{\lambda} g L_{B} L_{E} L_{J} m_{J} s_{5}-$ $2 A_{B}^{t} c_{\lambda} L_{E} L_{G B} L_{J} m_{B} s_{\alpha}-2 c_{12} c_{\lambda} g L_{E} L_{G B} L_{J} m_{B} s_{\alpha}+A_{J}^{t} L_{B} L_{E} m_{J} q_{6} s_{\lambda}+$ $g L_{B} L_{E} m_{J} q_{6} s_{5} s_{\lambda}-A_{B}^{t} L_{E} L_{G B} m_{B} q_{6} c_{\alpha} s_{\lambda}-c_{12} g L_{E} L_{G B} m_{B} q_{6} s_{\alpha} s_{\lambda}+$ $A_{E}^{t} L_{B} L_{G E} m_{E} q_{6} s_{\lambda}^{2}+c_{34} g L_{B} L_{G E} m_{E} q_{6} s_{\lambda}^{2}+c_{\alpha} c_{\lambda} I_{B} L_{E} q_{6} \dot{u}_{1}-$ $2 c_{\lambda} I_{B} L_{E} L_{J} s_{\alpha} \dot{u}_{1}-I_{B} L_{E} q_{6} s_{\alpha} s_{\lambda} \dot{u}_{1}+c_{\alpha} c_{\lambda} I_{B} L_{E} q_{6} \dot{u}_{2}-2 c_{\lambda} I_{B} L_{E} L_{J} s_{\alpha} \dot{u}_{2}-$ $I_{B} L_{E} q_{6} s_{\alpha} s_{\lambda} \dot{u}_{2}+c_{\lambda}^{2} I_{E} L_{B} q_{6} \dot{u}_{3}+s_{\lambda}^{2} I_{E} L_{B} q_{6} \dot{u}_{3}+c_{\lambda}^{2} I_{E} L_{B} q_{6} \dot{u}_{4}+$

$$
\begin{aligned}
\left.s_{\lambda}^{2} I_{E} L_{B} q_{6} \dot{u}_{4}+c_{\lambda} I_{H} L_{B} L_{E} \dot{u}_{5}+c_{\lambda} I_{J} L_{B} L_{E} \dot{u}_{5}\right) / \\
L_{B} L_{E}\left(2 L_{J} c_{\alpha} c_{\lambda}+q_{6} c_{\lambda} s_{\alpha}+q_{6} c_{\alpha} s_{\lambda}\right)
\end{aligned}
$$

\subsubsection{Forças de reação $F_{1}$ e $F_{2}$}

Para a determinação das forças de reação no atuador $O_{6}$, isto é, $F_{1}$ e $F_{2}$, e das forças $F_{3}$ e $F_{4}$ que atuam na junta $A$, utilizamos as equações 8.4, 8.5, 8.8 e a quarta equação foi obtida multiplicando-se escalarmente a equação 8.2 pelo vetor unitário $\overrightarrow{a_{1}}$. Isto é:

$$
\left[\left(F_{1}+F_{3}\right) \overrightarrow{a_{1}}+\left(F_{2}+F_{4}\right) \overrightarrow{a_{2}}-m_{A} g \overrightarrow{n_{2}}\right] \overrightarrow{a_{1}}=\left[m_{A}\left(A_{A}^{n} \overrightarrow{a_{1}}+A_{A}^{t} \overrightarrow{a_{2}}\right)\right] \overrightarrow{a_{1}}
$$

que desenvolvendo chegamos a

$$
F_{1}+F_{3}-m_{A} g s_{1}=m_{A} A_{A}^{n}
$$

No AnexoK apresentamos o programa Mathematica que deduz as equações destas forças.

\subsubsection{Forças de reação $F_{1}^{D}$ e $F_{2}^{D}$}

Para a determinação das forças de reação no atuador $O_{2}$, isto é, $F_{1}^{D}$ e $F_{2}^{D}$, e das forças $F_{3}^{D}$ e $F_{4}^{D}$ que atuam na junta $D$, utilizamos as equações 8.16, 8.17, 
8.20 e a quarta equação foi obtida multiplicando-se escalarmente a equação 8.14 pelo vetor unitário $\overrightarrow{d_{1}}$. Isto é:

$$
\left[\left(F_{1}^{D}+F_{3}^{D}\right) \overrightarrow{d_{1}}+\left(F_{2}^{D}+F_{4}^{D}\right) \overrightarrow{d_{2}}-m_{D} g \overrightarrow{n_{2}}\right] \overrightarrow{d_{1}}=\left[m_{D}\left(A_{D}^{n} \overrightarrow{d_{1}}+A_{D}^{t} \overrightarrow{d_{2}}\right)\right] \overrightarrow{d_{1}}
$$

que desenvolvendo temos

$$
F_{1}^{D}+F_{3}^{D}-m_{D} g s_{3}=m_{D} A_{D}^{n}
$$

No AnexoL apresentamos o programa Mathematica que deduz as equações destas forças.

\subsubsection{Forças e torque de reação na cadeia passiva}

Para a determinação das forças e torque de reação na cadeia passiva, isto é, $F_{1}^{H}, F_{2}^{H}, F_{3}^{H}$ e $T_{4}$ utilizamos as equações $8.28,8.29,8.31$ e a quarta equação é obtida pelo produto escalar entre 8.26 e o vetor unitário $\overrightarrow{h_{1}}$, que dá:

$$
F_{3}^{H}=m_{H} g s_{7}+m_{H} A_{H}^{n}
$$

No AnexoM apresentamos o programa Mathematica que deduz as equações destes esforços.

\subsection{Roteiro de cálculo}

Agora que temos a nossa disposição os recursos e as equações necessárias para o cálculo das reações nos atuadores e nas juntas do mecanismo, podemos resumir nosso roteiro de cálculo, conforme os seguintes passos:

\section{Primeiro passo: Ângulos dos elos. Anexo A ou B.}

Neste primeiro passo devemos usar os programas que foram desenvolvidos anteriormente no Capítulo 3: Análise cinemática: posições. É importante salientar, entretanto, que as designações então usadas para os dados e variáveis do mecanismo são diferentes das que estamos usando agora na análise dinâmica. Infelizmente isto foi necessário para dar maior clareza na aplicação do método de Kane. Temos duas situações a serem consideradas neste primeiro passo:

a) - Na cinemática inversa devemos entrar com os comprimentos dos elos $L_{1}$ a $L_{6}$ e com a posição da garra dada pelo comprimento $L_{7}$ e o ângulo $\theta_{7}$. O programa 
que calcula os demais ângulos dos elos é mostrado no Anexo A .

b) - Na cinemática direta são dados os ângulos de entrada e calculamos a posição da garra dada pelo comprimento $L_{7}$ e o ângulo $\theta_{7}$. Neste caso devemos usar o programa mostrado no Anexo B.

\section{Segundo passo: Espaço de trabalho. Anexo C.}

Através do programa do Anexo $\mathrm{C}$ podemos verificar se o espaço de trabalho atende nossas necessidades.

\section{Terceiro passo: Trajetória e movimento da garra. Anexos D, E e F.}

Com os programas dos Anexos D, E e F determinamos as componentes da velocidade e aceleração da garra do mecanismo em trajetória retilínea ou circular. Estes resultados serão úteis para o desenvolvimento do próximo passo.

\section{Quarto passo: Velocidades e acelerações dos elos. Anexo H}

No Anexo H temos o programa onde entrando-se com os ângulos e dimensões do mecanismo, posições dos CG e dados do movimento da garra, calculamos todas velocidades e acelerações angulares dos elos, $u_{1}=\dot{\theta_{1}}, \dot{u_{1}}=\ddot{\theta_{1}}, u_{2}=\dot{\theta_{2}}, \dot{u_{2}}=\ddot{\theta_{2}}$, etc. e as acelerações dos CG dos elos, $A_{A}^{n}, A_{A}^{t}, A_{B}^{n}$, etc. Estes resultados serão necessários no desenvolvimento do Anexo N.

\section{Quinto passo: Reações estáticas. Anexo I}

No Anexo I temos o programa que, fornecendo-se as dimensões do mecanismo, os pesos das peças e dos contrapesos, as posições dos seus centros de gravidade, a força externa e o momento externo aplicados, determina estaticamente as forças e torques dos atuadores bem como a força de reação na junta de rotação da cadeia passiva. Além disso, o programa verifica e informa as singularidades eventualmente existentes e traça um gráfico do mecanismo.

\section{Sexto passo: Torques dinâmicos de reação nos atuadores. Anexo N}

No Anexo $\mathrm{N}$ entramos com os dados determinados em passos anteriores e o programa calcula os torques de reação $T_{1}$ e $T_{1}^{D}$. Neste caso devemos acrescentar os dados de entrada: aceleração da gravidade, massas e momentos de inércia dos elos, os quais serão também necessários nos passos seguintes. 


\section{Sétimo passo: Forças dinâmicas de reação das cadeias ativas. Anexo O}

O Anexo $\mathrm{O}$ mostra o programa que calcula as forças $F_{1}$ a $F_{6}$ e $F_{1}^{D}$ a $F_{6}^{D}$. São forças de reação dinâmica nos atuadores as forças $F_{1}, F_{2}, F_{1}^{D}$ e $F_{2}^{D}$. Todas demais são forças nas juntas. Neste programa devemos entrar também com os torques calculados no passo anterior.

\section{Oitavo passo: Forças e torque dinâmicos na cadeia passiva. Anexo $\mathbf{P}$}

No Anexo P temos o programa que calcula os valores numéricos das forças de reação $F_{1}^{H}$ e $F_{3}^{H}$ na junta de rotação da cadeia passiva, alem da força $F_{2}^{H}$ e do torque $T_{4}$ que atuam na extremidade superior da cadeia passiva. Neste programa devemos acrescentar a entrada das seguintes forças calculadas no passo anterior: $F_{5}, F_{5}^{D}, F_{6}$ e $F_{6}^{D}$.

\subsubsection{Aglutinação dos programas. Anexo Q}

Para o cálculo dos torques e forças de reação nos atuadores agrupamos vários passos anteriores (utilizando a opção Cinemática Inversa) em uma só listagem, conforme apresentado no Anexo Q . Salientamos, entretanto, que este programa não leva em conta os contrapesos.

Para usar este programa devemos entrar com os seguintes dados:

-Comprimento, massa, momento de inércia e posição do CG dos elos.

-Posição da garra (ponto $J^{*}$ ), sua velocidade e aceleração.

-Momento externo aplicado na garra.

-Força externa aplicada na garra e seu ângulo com a horizontal.

- Valor da aceleração da gravidade.

Este programa determina entre outros esforços os seguintes:

1- $\left(M_{2}, M_{8}\right)$ : Torques estáticos de reação respectivamente nos atuadores $\mathrm{O}_{2}$ e $O_{6}$ levando-se em conta somente os esforços na garra $(F$ e $M)$.

2- $\left(T_{1 D}, T_{1}\right)$ : Torques dinâmicos de reação respectivamente nos atuadores $O_{2}$ e $O_{6}$ levando-se em conta somente os efeitos das massas dos elos.

3- $\left(M_{O 2}, M_{O 6}\right)$ : Torques finais (soma dos torques anteriores) respectivamente nos atuadores $\mathrm{O}_{2}$ e $\mathrm{O}_{6}$ conforme mostra a fig. 8.5. 


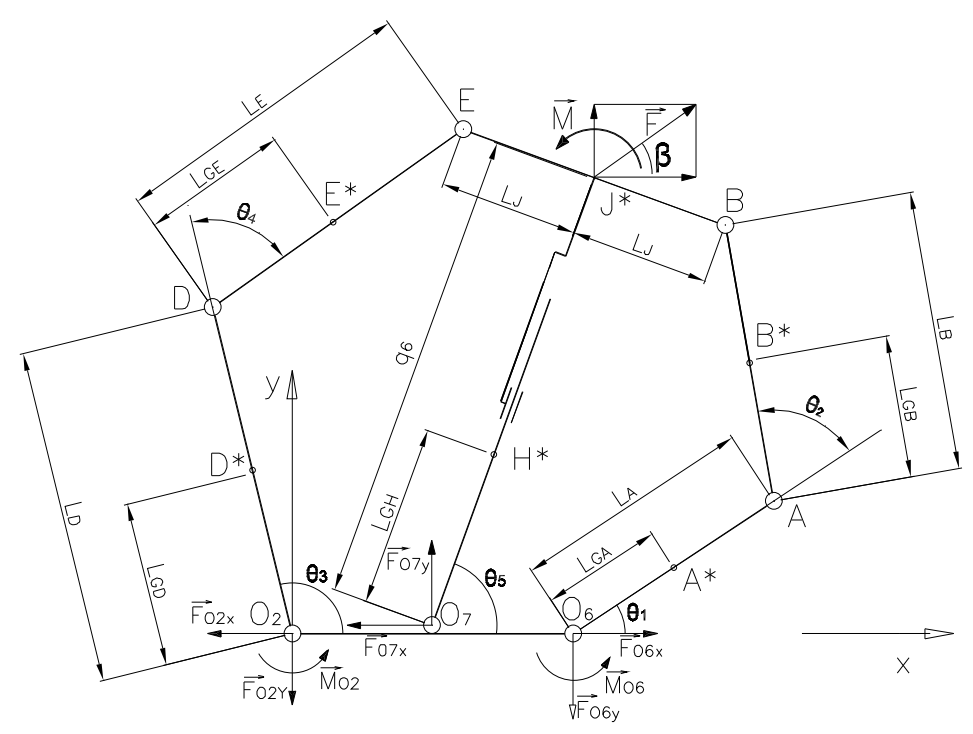

Figura 8.5: Mecanismo: reações finais nos atuadores

\subsubsection{Exemplo de aplicação 1}

Afim de melhor ilustrarmos o problema vamos usar o programa do Anexo Q para determinar os torques de reação dinâmicos $T_{1}^{D}$ e $T_{1}$ em função da variação discreta da posição garra (ponto $J^{*}$ ). Neste exemplo vamos supor que a garra se desloque verticalmente com velocidade constante de $1 \mathrm{~m} / \mathrm{s}$.

Os dados de entrada são os seguintes:

-Comprimento dos elos: $0,4 \mathrm{~m}$

-Massa de cada elo: $1 \mathrm{~kg}$

-Momento de inércia dos elos em relação aos seus CG: $0.013 \mathrm{~kg} \cdot \mathrm{m}^{2}$

-Posições do ponto $J^{*}$ : consideraremos o ângulo $\theta_{5}=\frac{\pi}{2}$ e $q_{6}$ variando de $0,4 m$ a $0,7 \mathrm{~m}$ em intervalos de $0,1 \mathrm{~m}$.

-Velocidade de $J^{*}: V_{J}^{y}=1 \mathrm{~m} / \mathrm{s}$ (não há componente horizontal)

-Aceleração de $J^{*}$ : inexistente.

-Força e momento na garra: inexistentes.

-Aceleração da gravidade: $g=10 \mathrm{~m} / \mathrm{s}^{2}$.

Os torques resultantes (em $\mathrm{Nm}$ ) foram:

\begin{tabular}{ccccc}
\hline$q_{6}$ & 0,4 & 0,5 & 0,6 & 0,7 \\
\hline$T_{1}^{D}$ & $-10,52$ & $-9,58$ & $-8,37$ & $-7,09$ \\
$T_{1}$ & 10,52 & 9,58 & 8,37 & 7,09 \\
\hline
\end{tabular}

Tabela 8.1: Exemplo de aplicação 1. Análise dinâmica 
No próximo capítulo, na fig. 9.2, mostramos as curvas correspondentes a estes valores e também as curvas para a mesma situação, mas considerando-se a existência de força de atrito na junta prismática.

\subsubsection{Exemplo de aplicação 2}

No exemplo anterior mantivemos constante o ângulo da cadeia passiva e variamos seu comprimento; e consideramos também que a garra (ponto $J^{*}$ ) possuía uma velocidade vertical unitária. Neste exemplo 2 vamos manter constante o comprimento da cadeia passiva e vamos variar o seu ângulo de inclinação.

Então todos os dados de entrada são os mesmos do exemplo anterior, exceto os seguintes:

-Posições do ponto $J^{*}$ : consideraremos $q_{6}=0,5$ e o ângulo $\theta_{5}$ inicialmente igual a $90^{\circ}$ e em seguida decrescendo de $10^{\circ} \mathrm{em} 10^{\circ}$.

O resultado dos torques de reação dinâmicos foi o seguinte:

\begin{tabular}{llllll}
\hline$\theta_{5}$ & $90^{\circ}$ & $80^{\circ}$ & $70^{\circ}$ & $60^{\circ}$ & $50^{\circ}$ \\
\hline$T_{1}^{D}$ & $-9,58$ & $-8,53$ & $-7,01$ & $-5,11$ & $-2,96$ \\
$T_{1}$ & 9,58 & 10,13 & 10,15 & 9,71 & 8,97 \\
\hline
\end{tabular}

Tabela 8.2: Exemplo de aplicação 2. Análise dinâmica

Veja no próximo capítulo na fig.9.3 as curvas correspondentes.

\subsubsection{Exemplo de aplicação 3}

Neste exemplo vamos considerar que a garra descreva uma trajetória circular passando pelas três fases de movimento: aceleração, velocidade constante e desaceleração.

Suponhamos um mecanismo onde cada elo tem massa de $1 \mathrm{~kg}$, comprimento de $0,4 m$ e momento de inércia em relação ao seu centro de gravidade igual a $0,013 \mathrm{~kg} \cdot \mathrm{m}^{2}$, e aceleração da gravidade $10 \mathrm{~m} / \mathrm{s}^{2}$. São dados de projeto a velocidade tangencial máxima de $1 \mathrm{~m} / \mathrm{s}$ e o módulo de aceleração e desaceleração tangencial de $10 \mathrm{~m} / \mathrm{s}^{2}$. Suponhamos ainda que a garra do mecanismo, não sujeita a força externa, parta do ponto $A(0,211 ; 0,453)$ e pare no ponto $D(0 ; 0,5)$ descrevendo um arco no sentido anti-horário de raio $0,5 \mathrm{~m}$ com centro na origem dos eixos de coordenadas conforme a fig. 8.6. Pede-se determinar os momentos de reação nos atuadores, $T_{1 D}$ e $T_{1}$, para 9 pontos intermediários da trajetória $A D$, igualmente distantes entre si. 


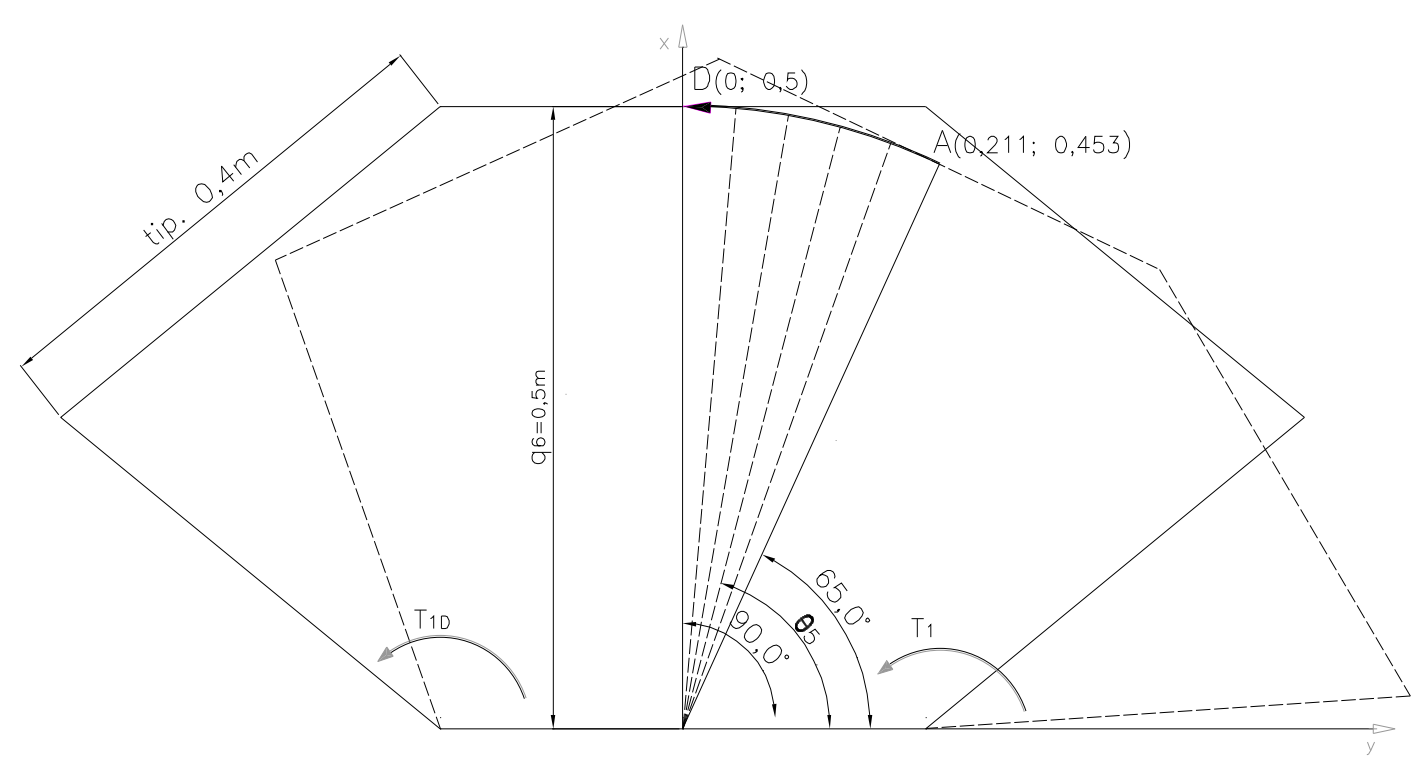

Figura 8.6: Exemplo 3: trajetória da garra

Para a solução deste problema primeiro devemos determinar as componentes das velocidades e acelerações dos 9 pontos igualmente espaçados da trajetória $A D$, utilizando o Anexo F. Utilizando o Anexo Q, para cada posição da garra, calculamos os torques nos atuadores, conforme mostrado na tabela seguinte:

\begin{tabular}{ccccccccc}
\hline Pos. & $\theta_{5}$ & $\mathrm{v}_{J x}$ & $\mathrm{v}_{J y}$ & $\mathrm{a}_{J x}$ & $\mathrm{a}_{J y}$ & $\mathrm{~T}_{1 D}$ & $\mathrm{~T}_{1}$ & Regime \\
\hline 1 & $67.5^{\circ}$ & $-0,61$ & 0,25 & $-9,57$ & 3,02 & 1,82 & 18,69 & acel. \\
2 & $70^{\circ}$ & $-0,88$ & 0,32 & $-9,99$ & 1,78 & 2,09 & 18,03 & acel. \\
3 & $72.5^{\circ}$ & $-0,95$ & 0,30 & $-0,60$ & $-1,91$ & $-5,32$ & 9,26 & $\mathrm{v}=$ cte. \\
4 & $75^{\circ}$ & $-0,96$ & 0,26 & $-0,52$ & $-1,93$ & $-5,75$ & 9,15 & $\mathrm{v}=$ cte. \\
5 & $77.5^{\circ}$ & $-0,97$ & 0,22 & $-0,43$ & $-1,95$ & $-6,17$ & 9,01 & $\mathrm{v}=$ cte. \\
6 & $80^{\circ}$ & $-0,98$ & $-0,17$ & $-0,35$ & $-1,97$ & $-6,56$ & 8,84 & $\mathrm{v}=$ cte. \\
7 & $82.5^{\circ}$ & -0.99 & 0,13 & $-0,26$ & $-1,98$ & $-6,93$ & 8,64 & v=cte. \\
8 & $85^{\circ}$ & $-0,93$ & 0,08 & 9,81 & $-2,61$ & $-15,19$ & 0,51 & desac. \\
9 & $87.5^{\circ}$ & $-0,66$ & 0,03 & 9,95 & $-1,31$ & $-16,25$ & 0,99 & desac. \\
\hline
\end{tabular}

Tabela 8.3: Exemplo de aplicação 3. Análise dinâmica

Lançando os resultados dos torques $T_{1 D}$ e $T_{1}$ num gráfico, obteremos as seguintes curvas: 


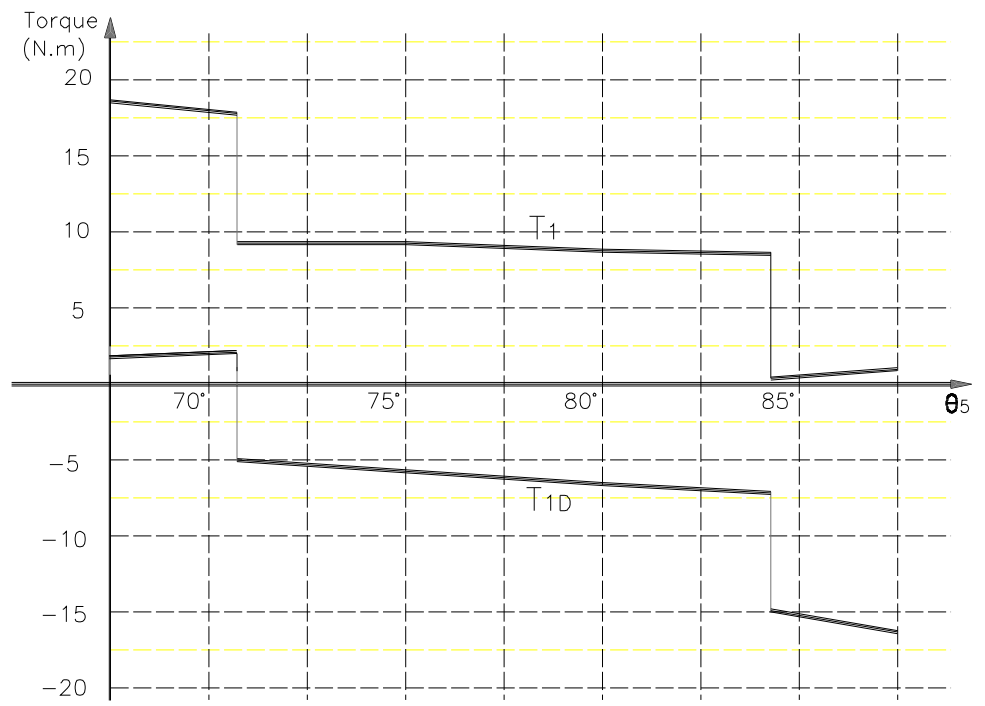

Figura 8.7: Exemplo 3: curvas dos torques nos atuadores 


\section{Capítulo 9: EFEITO DO ATRITO NAS JUNTAS}

\subsection{Introdução}

A força de atrito é a força que se opõe ao movimento dos corpos, causada pela força de contato entre eles e pelas propriedades de suas áreas de contato no que se refere á rugosidade, rigidez dos materiais, lubrificação, bem como de suas velocidades relativas. Face a esta diversidade de propriedades que influem na determinação do atrito definem-se vários tipos de força de atrito.

O atrito estático é a força necessária para iniciar o movimento. O atrito de Coulomb é a componente do atrito que não depende da magnitude da velocidade, enquanto que o atrito viscoso depende da magnitude da velocidade. Existe ainda um modelo de força de atrito chamado de Karnopp que leva em conta o afundamento (stick) e o escorregamento (slip) correspondentes ao início do movimento do corpo e sua velocidade posterior. O efeito de Stribeck é a diminuição do atrito com o aumento da velocidade em baixas velocidades.

Uma avaliação da força de atrito pode ser obtida pela soma do atrito de Coulomb e do atrito viscoso, isto é:

$$
F_{a}=C \cdot \operatorname{sign}(\dot{q})+V
$$

Onde $F_{a}$ é a resultante da força de atrito; $C$ é o atrito de Coulomb, $V$ o atrito viscoso e $\dot{q}$ a velocidade. Sendo

$$
C=k N
$$

para juntas de translação.

$$
C=k_{r} \frac{d}{2} N
$$

para juntas de rotação. E

$$
V=c \dot{q}
$$

Onde

$k$ é o coeficiente de atrito de Coulomb,

$k_{r}$ é o coeficiente de atrito de rolamento,

$d$ é o diâmetro da junta de rotação e

$c$ é o coeficiente de atrito viscoso.

O artigo [19] de Itul; Pisla desenvolve o estudo de um modelo de atrito para um robô paralelo considerando os efeitos do atrito de Coulomb e do atrito viscoso. Neste artigo é mencionado que poucos autores têm considerado o efeito do atrito 
e que este efeito pode representar cerca de $25 \%$ das forças/torques necessários para a movimentação de um manipulador em situações típicas [20]. O trabalho de Farhat; Diaz; Mata [21] apresenta um modelo de atrito não linear: são considerados os atritos de Coulomb, o atrito estático, o atrito viscoso e a parcela não linear referente à velocidade de Stribeck

Asada; Slotine [22] e Craig [20] destacam que a preponderância do atrito é devida à redução por pares de engrenagens entre o motor e o braço do mecanismo. Não há menção a respeito do atrito nas juntas, dando a entender que sua influência é pequena.

No nosso trabalho, entretanto, estamos interessados na determinação dos torques diretamente atuantes nos braços do mecanismo. Assim sendo vamos considerar a existência de atrito na junta prismática e vamos desprezar o atrito nas juntas de rotação já que o atrito nestas é normalmente muito menor que naquela. Vamos também desprezar a parcela de atrito viscoso, enfatizando somente o atrito de Coulomb.

\subsection{Desenvolvimento das equações}

Como vamos considerar somente o efeito da força de atrito da junta prismática $\left(F_{a}\right)$, podemos utilizar todas equações deduzidas no capítulo anterior exceto às referentes à cadeia passiva (elos $H$ e $J$ ).

Para maior abrangência vamos considerar também o efeito do torque $(\vec{M})$ e força $(\vec{F})$ atuantes na garra, além dos pesos próprios das partes.

A fig. 9.1 mostra os novos diagramas de corpo livre dos elos $H$ e $J$, tendo em vista as considerações acima e a seguir desenvolveremos as novas equações para estes elos.

\subsubsection{Elo $\mathbf{H}$}

Em relação ao capítulo anterior só houve mudança no TMB do elo H que ficou assim:

\section{TMB do elo $\mathbf{H}$}

$$
F_{1}^{H} \overrightarrow{h_{2}}+F_{2}^{H} \overrightarrow{h_{2}}+F_{3}^{H} \overrightarrow{h_{1}}-F_{a} \overrightarrow{h_{1}} \operatorname{sign}\left(\dot{q}_{6}\right)-m_{H} g \overrightarrow{n_{2}}=m_{H}\left(A_{H}^{n} \overrightarrow{h_{1}}+A_{H}^{t} \overrightarrow{h_{2}}\right)
$$



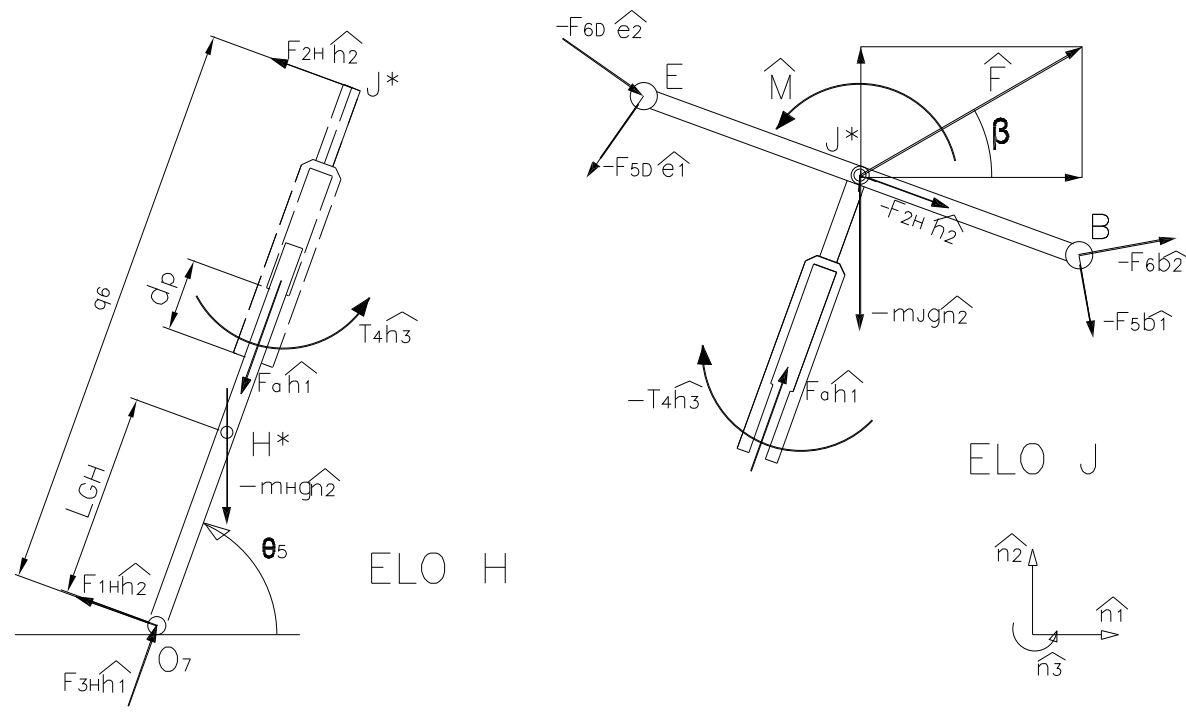

Figura 9.1: Diagramas dos elos $\mathrm{H}$ e J

\subsubsection{Elo J}

Em relação ao elo J as seguintes equações foram alteradas:

\section{TMB do elo J}

$$
\begin{gathered}
-F_{6}^{D} \overrightarrow{e_{2}}-F_{5}^{D} \overrightarrow{e_{1}}-F_{2}^{H} \overrightarrow{h_{2}}-F_{5} \overrightarrow{b_{1}}-F_{6} \overrightarrow{b_{2}}-m_{J} g \overrightarrow{n_{2}}+F c \beta \overrightarrow{n_{1}}+ \\
F s \beta \overrightarrow{n_{2}}-\operatorname{sign}\left(\dot{q}_{6}\right) F_{a} \overrightarrow{h_{1}}=m_{J}\left(A_{J}^{n} \overrightarrow{h_{2}}+A_{J}^{t} \overrightarrow{h_{1}}\right)
\end{gathered}
$$

\section{TMA do elo J}

$$
\left(-T_{4}+L_{J} F_{6}^{D} s_{\lambda}+L_{J} F_{5}^{D} c_{\lambda}-L_{J} F_{5} c_{\alpha}+L_{J} F_{6} s_{\alpha}+M\right) \overrightarrow{h_{3}}=I_{J} \dot{u_{5}} \overrightarrow{h_{3}}
$$

onde

$$
\begin{array}{ll}
s_{\lambda}=\operatorname{sen}\left(\theta_{5}-\theta_{4}-\theta_{3}\right) & c_{\lambda}=\cos \left(\theta_{5}-\theta_{4}-\theta_{3}\right) \\
s_{\alpha}=\operatorname{sen}\left(\theta_{1}+\theta_{2}-\theta_{5}\right) & c_{\alpha}=\cos \left(\theta_{1}+\theta_{2}-\theta_{5}\right)
\end{array}
$$

\section{Equação (JF5)}

$$
\begin{gathered}
-F_{6}^{D} q_{6} c_{\lambda}+F_{5}^{D} q_{6} s_{\lambda}-F_{2}^{H} q_{6}-F_{5} q_{6} s_{\alpha}-F_{6} q_{6} c_{\alpha}-m_{J} g q_{6} c_{5}- \\
F c \beta q_{6} s_{5}+F s \beta q_{6} c_{5}=m_{J} q_{6} A_{J}^{n}
\end{gathered}
$$


Equação (JM5)

$$
-T_{4}+L_{J} F_{6}^{D} s_{\lambda}+L_{J} F_{5}^{D} c_{\lambda}-L_{J} F_{5} c_{\alpha}+L_{J} F_{6} s_{\alpha}+M=I_{J} \dot{u}_{5}
$$

Equação (JF6)

$$
\begin{gathered}
-F_{6}^{D} s_{\lambda}-F_{5}^{D} c_{\lambda}-F_{5} c_{\alpha}+F_{6} s_{\alpha}-m_{J} g s_{5}+F c \beta c_{5}+ \\
F s \beta s_{5}-\operatorname{sign}\left(\dot{q}_{6}\right) F_{a}=m_{J} A_{J}^{t}
\end{gathered}
$$

\subsubsection{Resultado para os elos $\mathbf{H}$ e $\mathbf{J}$}

Em consequiência das alterações acima, as equações (HJ5) e (HJ6) modificaramse.

$$
\text { 1. Equação }(\text { HJ5 })=(\text { HF5 })+(\text { HM5 })+(\text { JF5 })+(\text { JM5 })
$$

$$
\begin{gathered}
-F_{5}\left(L_{j} c_{\alpha}+q_{6} s_{\alpha}\right)+F_{5}^{D}\left(L_{j} c_{\lambda}+q_{6} s_{\lambda}\right)+F_{6}\left(L_{j} s_{\alpha}-q_{6} c_{\alpha}\right)+F_{6}^{D}\left(L_{j} s_{\lambda}-q_{6} c_{\lambda}\right)- \\
-L_{G H} m_{H} g c_{5}-m_{J} g q_{6} c_{5}-F c \beta q_{6} s_{5}+F s \beta q_{6} c_{5}+M \\
=L_{G H} m_{H} A_{H}^{t}+m_{J} q_{6} A_{J}^{n}+\left(I_{H}+I_{J}\right) \dot{u}_{5}
\end{gathered}
$$

2. Equação $(\mathrm{HJ6})=(\mathrm{HF} 6)+(\mathrm{HM} 6)+(\mathrm{JF} 6)+(\mathrm{JM} 6)$

$$
\begin{gathered}
-F_{6}^{D} s_{\lambda}-F_{5}^{D} c_{\lambda}-F_{5} c_{\alpha}+F_{6} s_{\alpha}-m_{J} g s_{5}+ \\
F c \beta c_{5}+F s \beta s_{5}-\operatorname{sign}\left(\dot{q}_{6}\right) F_{a}=m_{J} A_{J}^{t}
\end{gathered}
$$

\subsubsection{Força de atrito na junta prismática}

Como no nosso caso a junta prismática está sujeita somente ao torque $T_{4}$, a força de atrito nesta junta é calculada por

$$
F_{a}=\frac{T_{4}}{d_{p}} k_{p}+F_{p r}
$$

Onde $T_{4}$ é o torque atuante na junta prismática, $d_{p}$ e $k_{p}$ respectivamente seu comprimento e seu coeficiente de atrito; e $F_{p r}$ é a força de atrito da proteção da junta prismática. 


\subsubsection{Resultado geral}

Ajuntando-se as equações (AB1), (AB2), (DE3) e (DE4) do capítulo anterior mais as equações (HJ5), (HJ6) que deduzimos neste capítulo e a equação da força de atrito acima, isto é, agrupando-se as equações 8.12, 8.13, 8.24, 8.25, 9.1, 9.2 e 9.3 podemos determinar os torques $T_{1}$ e $T_{1 D}$ nos atuadores, o torque $T_{4}$ na junta prismática e as forças $F_{a}, F_{5}, F_{6}, F_{5 D}$ e $F_{6 D}$.

No Anexo R apresentamos o programa que deduz as equações para o cálculo destas incógnitas.

No Anexo S apresentamos o programa que, utilizando as equações deduzidas no Anexo $\mathrm{R}$, calcula os torques de reação nos atuadores, $T_{1}$ e $T_{1 D}$. Neste programa além dos parâmetros do mecanismo, velocidades e acelerações da garra e propriedades do atrito da junta prismática, podemos entrar também com força e momento atuantes na garra.

\subsubsection{Exemplo de aplicação 1}

Vamos pegar o exemplo de aplicação 1 do capítulo anterior e vamos calcular os torques $T_{1 D}$ e $T_{1}$ nos atuadores, porém agora considerando a existência de atrito na junta prismática com as seguintes características:

-Comprimento da junta prismática $d_{p}=0,07 m$

-Coeficiente de atrito na junta prismática $k_{p}=0,2$

-Força de atrito da proteção da junta prismática $F_{\text {prot }}=5 \mathrm{~N}$

Utilizando o Anexo S chegamos ao seguinte resultado:

\begin{tabular}{ccccc}
\hline$q_{6}$ & 0,4 & 0,5 & 0,6 & 0,7 \\
\hline$T_{1 D}$ & $-12,25$ & $-11,15$ & $-9,69$ & $-8,05$ \\
$T_{1}$ & 12,25 & 11,15 & 9,69 & 8,05 \\
\hline
\end{tabular}

Tabela 9.1: Exemplo de aplicação 1. Atrito

A fig. 9.2 mostra as curvas dos torques acima e dos torques sem o atrito na junta prismática determinados no capítulo anterior. 


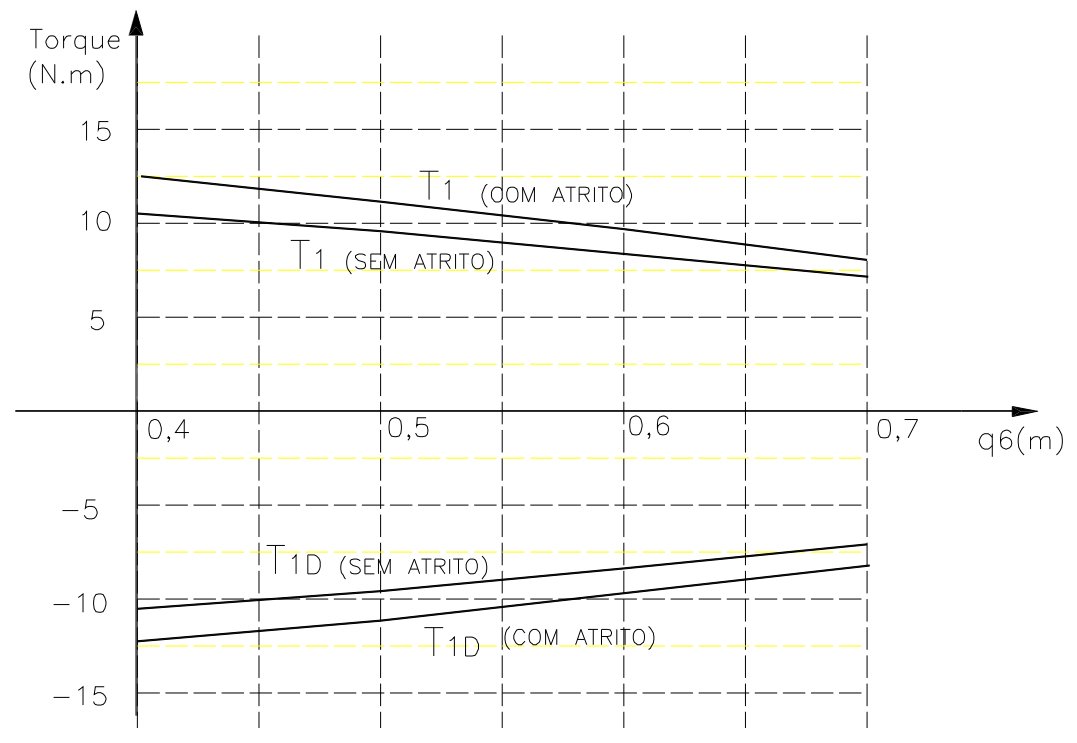

Figura 9.2: Exemplo 1: curvas dos torques com e sem atrito

\subsubsection{Exemplo de aplicação 2}

Vamos pegar o exemplo de aplicação 2 do capítulo anterior e vamos calcular os torques $T_{1 D}$ e $T_{1}$ nos atuadores, porém agora, considerando a existência de atrito na junta prismática com as mesmas características acima. Utilizando o Anexo S chegamos ao seguinte resultado:

\begin{tabular}{cccccc}
\hline$\theta_{5}$ & $90^{\circ}$ & $80^{\circ}$ & $70^{\circ}$ & $60^{\circ}$ & $50^{\circ}$ \\
\hline$T_{1 D}$ & $-11,15$ & $-10,26$ & $-8,93$ & $-7,27$ & $-5,40$ \\
$T_{1}$ & 11,15 & 11,73 & 11,78 & 11,40 & 10,74 \\
\hline
\end{tabular}

Tabela 9.2: Exemplo de aplicação 2. Atrito

A fig. 9.3 mostra as curvas dos torques acima e dos torques sem o atrito na junta prismática determinados no capítulo anterior. 


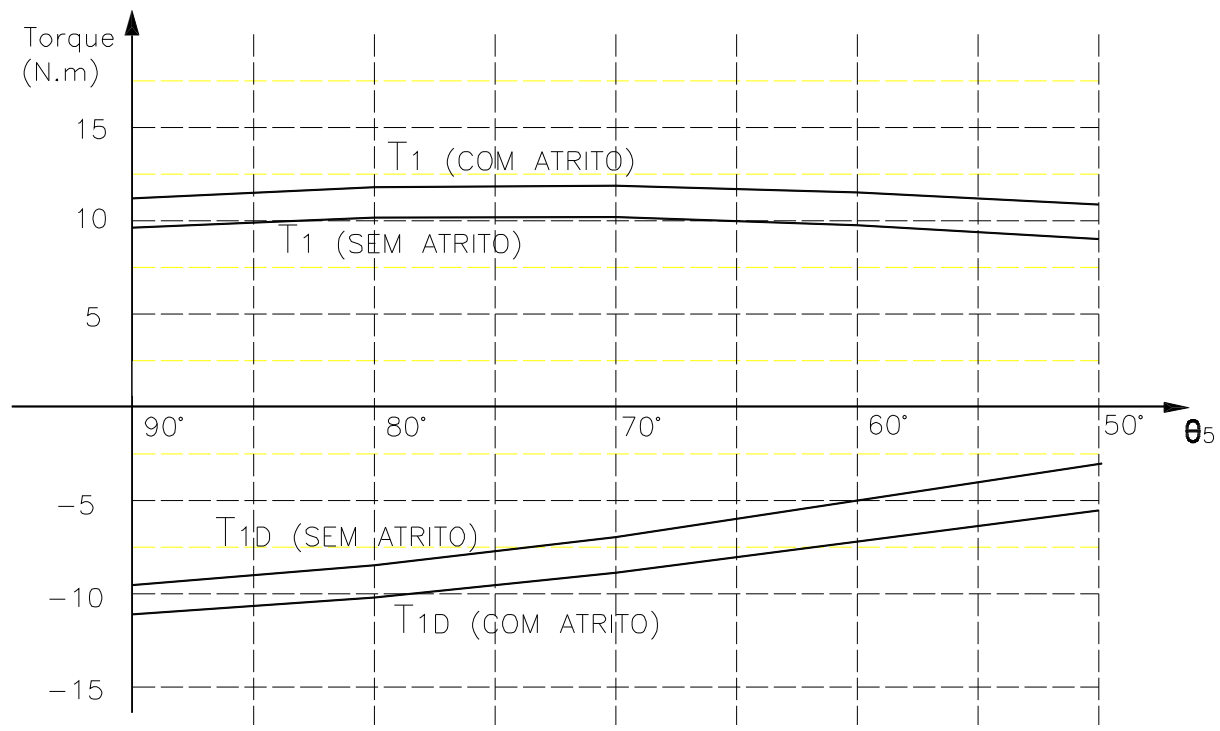

Figura 9.3: Exemplo 2: curvas dos torques com e sem atrito 


\section{Capítulo 10: DISCUSSÃO}

\subsection{Análise cinemática: posições}

\subsubsection{Cinemática inversa}

No estudo da cinemática inversa nós vimos que na determinação dos ângulos $\theta_{2}$ e $\theta_{6}$ teríamos duas soluções para cada ângulo, ou seja, o vértice $A$ poderia estar em $A^{\prime}$ ou $A^{\prime \prime}$ que são simétricos em relação à reta $O_{2} B$, o mesmo acontecendo com o vértice $D$ em relação à reta $O_{6} C$ (fig.10.1).

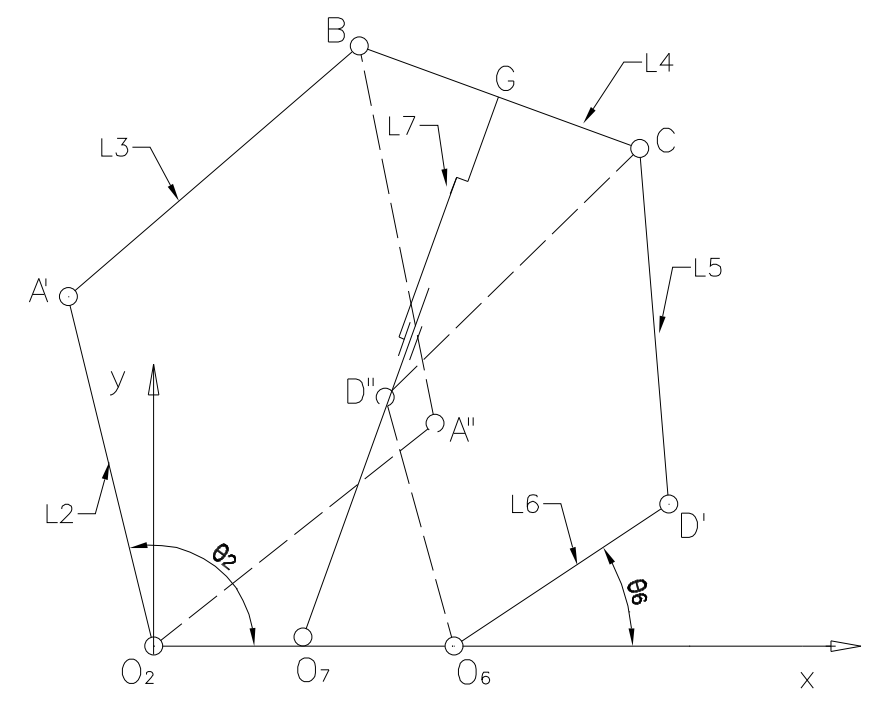

Figura 10.1: Posições das juntas A e D

Quando desenvolvemos o programa do Anexo A impomos a condição de que seja escolhido, para ambos os casos, somente o ponto situado do lado de fora em relação àquelas retas e, desta forma, estaremos evitando qualquer possível interferência das cadeias ativas com a cadeia passiva. E para isto, adotamos a propriedade do produto vetorial a qual nos parece muito útil para a solução de problemas de interferência de elos nos mecanismos e que explicaremos a seguir. 


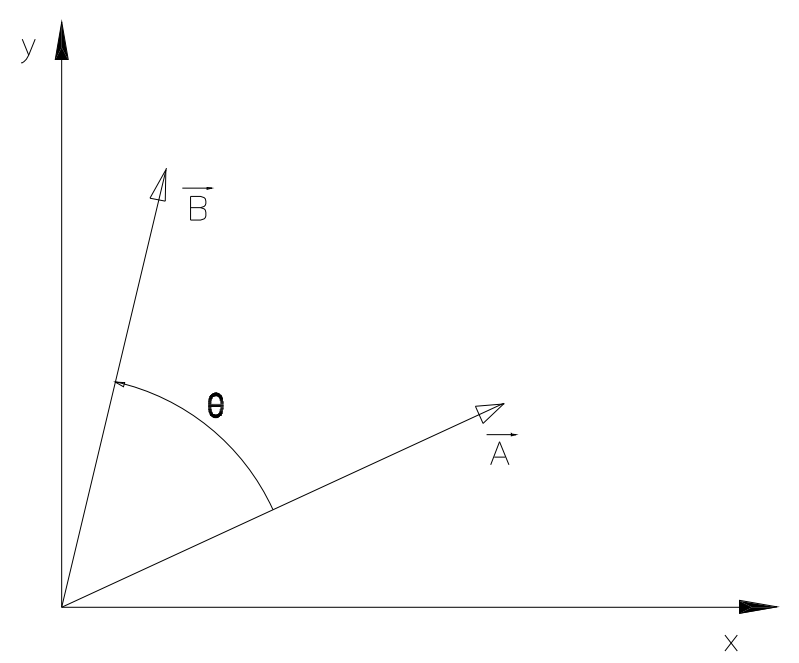

Figura 10.2: Propriedades do produto vetorial

Sejam dois vetores $\vec{A}=A_{x} \vec{i}+A_{y} \vec{j}+A_{z} \vec{k}$ e $\vec{B}=B_{x} \vec{i}+B_{y} \vec{j}+B_{z} \vec{k}$ no plano $x y$, ou seja, com $A_{z}=B_{z}=0$. Assim, temos:

$$
\vec{A} \wedge \vec{B}=\left(A_{x} B_{y}-A_{y} B_{x}\right) \vec{k}
$$

Sendo $\theta$ o ângulo entre $\vec{A}$ e $\vec{B}$, medido de $\vec{A}$ para $\vec{B}$ na direção anti-horária (fig.10.2). Assim sendo, como $\vec{A}$ e $\vec{B}$ estão no plano, as propriedades geométricas do produto vetorial nos dão:

$$
\vec{A} \wedge \vec{B}=A B \operatorname{sen} \theta \vec{k}
$$

Onde $A$ é o comprimento do vetor $\vec{A}$ e $B$ o comprimento do vetor $\vec{B}$.

Comparando as equações acima, temos:

$$
A_{x} B_{y}-A_{y} B_{x}=A B \operatorname{sen} \theta
$$

Portanto:

1. Se $A_{x} B_{y}-A_{y} B_{x}=0$, então $\vec{B}$ está na reta determinada por $\vec{A}$.

2. Se $A_{x} B_{y}-A_{y} B_{x}>0$, então $\vec{B}$ está à esquerda, relativa a um observador voltado para a direção apontada por $\vec{A}$, da reta determinada por $\vec{A}$.

3. Se $A_{x} B_{y}-A_{y} B_{x}<0$, então $\vec{B}$ está à direita, relativa a um observador voltado para a direção apontada por $\vec{A}$, da reta determinada por $\vec{A}$. 


\subsubsection{Cinemática direta}

No caso da cinemática direta nós impomos além da condição de que a cadeia passiva não avance para baixo da base, impomos uma segunda condição de que a plataforma móvel não deve interferir com a base ou com os elos adjacentes a ela. No desenvolvimento do Anexo B resolvemos isto adotando a teoria seguinte.

Consideramos dois segmentos de reta, por exemplo, $P Q$ e $R S$ e desenvolvemos as equações que passam por estes dois pontos. Se não houver solução para o sistema destas duas equações, isto quer dizer que elas são paralelas. Mas havendo solução então teremos um ponto de interseção $I$ das duas retas. Entretanto esta interseção pode ocorrer de 3 formas diferentes (fig.10.3):

1. O ponto de interseção pertence aos dois segmentos de reta $P Q$ e $R S$.

2. O ponto de interseção pertence a um segmento de reta e ao prolongamento do outro segmento.

3. O ponto de interseção está situado nos prolongamentos dos dois segmentos de reta, portanto, não pertence a nenhum dos dois segmentos de reta.
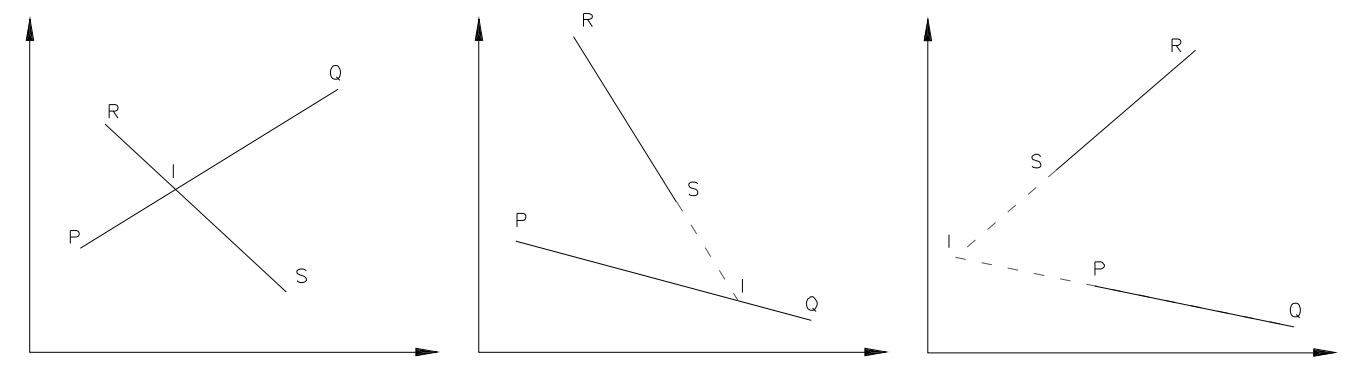

Figura 10.3: Interseção das retas PQ e RS

Para nós só interessa o primeiro caso, pois queremos justamente evitar que um elo de comprimento $P Q$ cruze como o outro elo de comprimento $R S$. E para que o ponto de interseção pertença aos dois segmentos é necessário que a sua abscissa (ou ordenada) esteja entre as abscissas (ou ordenadas) dos extremos dos segmentos $P Q$ e $R S$. 


\subsection{Análise dinâmica}

\subsubsection{Comparação entre o método de Kane com os de Newton-Euler e Lagrange}

Desenvolvemos a análise dinâmica inversa admitindo que os elos sejam corpos rígidos. Assim, não consideramos deslocamentos devidos à deformação elástica bem como vibrações mecânicas. Nosso intento foi a determinação dos torques nos atuadores ou dos esforços nas partes do mecanismo quando a garra do mesmo estivesse sujeita a movimentação e ação externa.

Adotamos o método de Kane na nossa análise dinâmica com o objetivo de conhecê-lo e termos uma idéia comparativa com os demais métodos.

Primeiramente vamos fazer a comparação com o método de Newton-Euler. Observamos que o modelo dinâmico do mecanismo, empregando o método de Newton-Euler, corresponde a um sistema linear contendo 18 equações e 18 incógnitas, a saber: 14 componentes de força nas juntas de rotação, 2 torques de reação (um para cada atuador) um torque e uma força na junta prismática da cadeia passiva. Ao adotarmos o método de Kane tivemos que desenvolver as equações do TMB e TMA para todas as partes do mecanismo, da mesma forma que faríamos se tivéssemos adotado o método de Newton-Euler.

A vantagem que pudemos observar no método de Kane em relação ao método de Newton-Euler foi que no final daquele método apareceram simplificações, resultando somente 6 equações e 6 incógnitas. Estas incógnitas eram os torques nos atuadores e as componentes das forças nas juntas de rotação da plataforma móvel. Portanto, se o nosso objetivo for somente o cálculo destes esforços não precisaremos desenvolver e resolver o sistema de 18 equações que o método de Newton-Euler exige.

Por outro lado, se pretendemos determinar somente os torques nos atuadores, podemos através de uma manipulação simbólica reduzir as seis equações obtidas pelo método de Kane em somente duas que nos fornecerão os dois torques.

Se o nosso objetivo for determinarmos todas as 18 incógnitas, pelo método de Kane já teríamos 6 incógnitas determinadas e utilizando as demais equações do TMB e TMA chegaremos às incógnitas restantes como, aliás, fizemos neste nosso trabalho.

Em resumo, no método de Kane utilizamos todas as equações do método de Newton-Euler e efetuamos o produto escalar com as derivadas parciais das velocidades lineares e angulares dos centros de massa de cada elo, em relação às variáveis de movimento e em seguida os agruparmos segundo estas variáveis. Isto 
pode parecer à primeira vista uma desvantagem em relação ao método de NewtonEuler, mas graças a este artifício esforços internos serão eliminados de forma idêntica ao que acontece com o método de Lagrange. Além disso, a experiência na aplicação do método de Kane vai fazer com que possamos perceber de antemão estas simplificações e o seu desenvolvimento poderá tornar-se ainda mais simples.

No desenvolvimento que fizemos para o método de Kane vimos que temos 6 peças móveis. Portanto tratamos o problema como se eu tivesse $r$ variando de 1 a 6 e estas 6 variáveis (5 são ângulos de elos e uma se refere ao comprimento da cadeia passiva) foram consideradas como independentes, de modo que não precisamos de aplicar a equação 8.1 para todos os elos mas somente para aqueles que implicitamente sabemos não serem nulos. Mas na realidade elas não são independentes e o vínculo entre elas é estabelecido pela trajetória da garra, ou seja, estarão vinculadas pela cinemática inversa de posição. Portanto quando calcularmos as acelerações dos baricentros elas irão respeitar esta condição de dependência. No final, com o método de Kane chegamos a seis equações.

Se aplicarmos o método de Kane a um sistema de duas peças móveis teríamos então duas variáveis. Ao calcularmos a aceleração do baricentro em função destas variáveis vamos chegar a duas equações do mesmo modo que chegaríamos se empregássemos o método de Lagrange. Podemos ver aí uma equivalência entre estes dois métodos.

Entretanto, ao empregarmos o método de Lagrange para o caso do nosso mecanismo, temos que desenvolver tudo em função de duas variáveis (ângulos dos atuadores). Inclusive a energia cinética e potencial dos seis elos devem ser desenvolvidas em função destas duas variáveis. Isto nos parece um problema verdadeiramente complexo ao contrário do que vimos no método de Kane.

Na tabela 10.1 apresentamos uma síntese comparativa entre os três métodos.

\begin{tabular}{llll}
\hline & Newton-Euler & Lagrange & Kane \\
\hline $\mathrm{N}^{o}$ de equações & 18 & 2 & $6^{*}$ \\
Custo computacional & Elevado & Pequeno & Médio \\
Dificuldade de obtenção & Fácil & Difícil & Média \\
\hline
\end{tabular}

* $\mathrm{N}^{o}$ de equações igual a 2 , com manipulação simbólica

Tabela 10.1: Comparação entre formulações

\subsubsection{O balanceamento com contrapesos}

No Capítulo 7, Seção 7.3, desenvolvemos as equações do cálculo dos contrapesos que anulam estaticamente os torques nos atuadores para a condição de que os 
braços dos contrapesos sejam iguais a 1/4 dos comprimentos dos respectivos elos. Entretanto, embora estaticamente possamos anular estes torques, dependendo das condições dinâmicas o efeito dos contrapesos poderá aumentá-los em vez de reduzi-los. É o que podemos verificar utilizando o Exemplo de aplicação 1 do Capítulo 8. Neste exemplo foi considerado que não existem nem contrapesos e nem esforços externos na garra e que a cadeia passiva permanece perpendicular à base, tendo porém seu comprimento variando conforme a velocidade dada. Vamos agora introduzir os contrapesos conforme definidos pelas equações da Seção 7.3 .

Temos então:

Massas dos elos (fig.10.4):

$m_{A *}=1 \mathrm{~kg}, m_{B *}=1 \mathrm{~kg}, m_{D *}=1 \mathrm{~kg}, m_{E *}=1 \mathrm{~kg}, m_{H *}=1 \mathrm{~kg}$ e $m_{J *}=1 \mathrm{~kg}$

Massas dos contrapesos:

$m_{w A}=24 k g, m_{w B}=4 k g, m_{w D}=24 k g, m_{w E}=4 k g$ e $m_{w H}=2 k g$.

Massas dos elos com contrapesos:

$m_{A}=25 \mathrm{~kg}, m_{B}=5 \mathrm{~kg}, m_{D}=25 \mathrm{~kg}, m_{E}=5 \mathrm{~kg}, m_{H}=3 \mathrm{~kg}$ e $m_{J}=1 \mathrm{~kg}$.

Posição dos CG dos elos com contrapesos:

$L_{G A}=-0,088 m, L_{G B}=-0,04 m, L_{G D}=-0,088 m, L_{G E}=-0,04 m \mathrm{e}$ $L_{G H}=0$.

Momentos de Inércia dos elos com contrapesos em relação ao novo CG:

$I_{A}=0,0994 \mathrm{~kg} \mathrm{~m}^{2}, I_{B}=0,085 \mathrm{~kg} \mathrm{~m}^{2}, I_{D}=0,0994 \mathrm{~kg} \mathrm{~m}^{2}$,

$I_{E}=0,085 \mathrm{kgm}^{2}, I_{H}=0,073 \mathrm{kgm}^{2}$ e $I_{J}=0,013 \mathrm{~kg} \mathrm{~m}^{2}$.

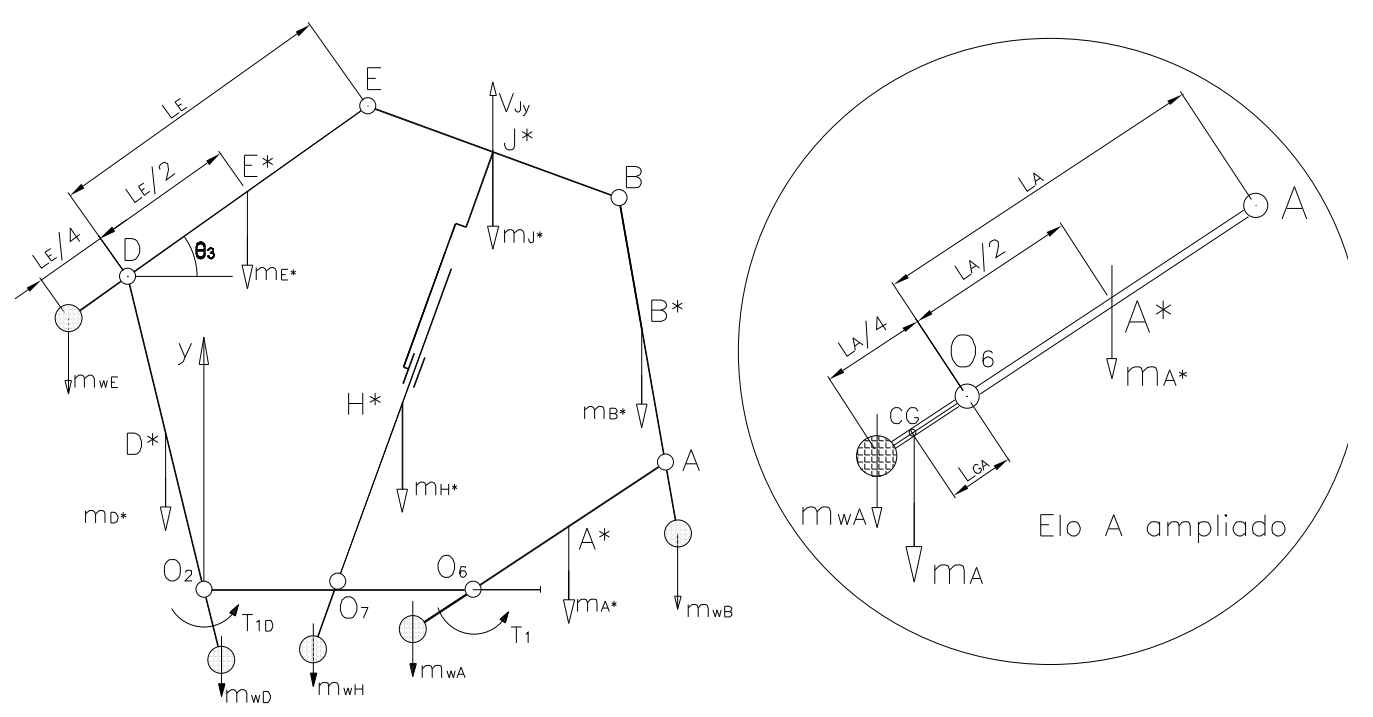

Figura 10.4: Balanceamento com contrapesos 
Utilizando os programas do Anexo Q ou Anexo S que fazem o cálculo dinâmico, vamos pegar a configuração do mecanismo tal que a sua cadeia passiva tenha o comprimento $q_{6}=0,5 \mathrm{~m}$ e vamos determinar os torques $T_{1}$ no atuador $O_{6}$ para várias velocidades da garra, considerando primeiro o mecanismo sem contrapesos e depois com contrapesos, conforme a tabela seguinte:

\begin{tabular}{cccccc}
\hline$V_{J y}(\mathrm{~m} / \mathrm{s})$ & 0 & 1 & 2 & 3 & 4 \\
\hline$T_{1}$ sem contrapesos $(N \mathrm{~m})$ & 9,37 & 9,58 & 10,24 & 11,33 & 12,85 \\
$T_{1}$ com contrapesos $(N \mathrm{~m})$ & 0 & 2,76 & 11,05 & 24,87 & 44,21 \\
\hline
\end{tabular}

Tabela 10.2: Influência dos contrapesos conforme a velocidade

Portanto podemos concluir por esta tabela que o balanceamento através de contrapesos é favorável até velocidades inferiores a $2 \mathrm{~m} / \mathrm{s}$. A partir daí o crescimento do torque, quando existem os contrapesos, é cada vez mais acentuado em relação ao crescimento do torque sem a existência de contrapesos. A grande vantagem do balanceamento pela utilização de molas é justamente pelo fato destas reduzirem as massas e, consequentemente, os seus efeitos de inércia, ao contrário do que acontece com os contrapesos.

\subsubsection{Influência das acelerações dos CG dos elos.}

Na seção anterior pudemos ter uma idéia da influência das massas e da velocidade sobre o valor dos torques nos atuadores. Surgem então outras questões. E quanto às acelerações dos CG dos elos? Até que ponto podemos desprezá-las nos nossos cálculos?

No Exemplo de aplicação 1 do Capítulo 8 foi considerado que a garra possui uma velocidade vertical de $1 \mathrm{~m} / \mathrm{s}$, que não existe nem força e nem momento externo atuando na garra, que a massa dos elos é de $1 \mathrm{~kg}$, etc. Para esta condição vamos construir a tabela seguinte que mostra os torques no atuador com e sem as acelerações do CG dos elos, para vários comprimentos $q_{6}$ da cadeia passiva.

\begin{tabular}{ccccc}
\hline$q_{6}(m)$ & 0,4 & 0,5 & 0,6 & 0,7 \\
\hline$T_{1}$ com acel. $(N m)$ & 10,52 & 9,58 & 8,36 & 7,09 \\
$T_{1}$ sem acel. $(N m)$ & 10,42 & 9,42 & 8,04 & 6,12 \\
$\Delta$ & 0,10 & 0,16 & 0,32 & 0,97 \\
$\%$ & 0,95 & 1,70 & 3,98 & 15,85 \\
\hline
\end{tabular}

Tabela 10.3: Torques com e sem acelerações e v=1m/s 
Portanto vemos que quanto maior for o comprimento da cadeia passiva maior a influência das acelerações e acima de $0,5 \mathrm{~m}$ já não mais podemos desprezar sua influência.

Vamos agora construir a tabela seguinte com os mesmos parâmetros da tabela anterior, mas mudando a velocidade da garra para $4 \mathrm{~m} / \mathrm{s}$ :

\begin{tabular}{ccccc}
\hline$q_{6}(m)$ & 0,4 & 0,5 & 0,6 & 0,7 \\
\hline$T_{1}$ com acel. $(N m)$ & 12,43 & 12,85 & 14,80 & 26,25 \\
$T_{1}$ sem acel. $(N m)$ & 10,89 & 10,22 & 9,62 & 10,82 \\
$\Delta$ & 1,54 & 2,63 & 5,18 & 15,43 \\
$\%$ & 14,14 & 25,73 & 53,84 & 142,60 \\
\hline
\end{tabular}

Tabela 10.4: Torques com e sem acelerações e v $=4 \mathrm{~m} / \mathrm{s}$

Agravou-se muito mais a influência das acelerações não havendo condição de desprezá-la em nenhum dos casos.

\subsubsection{A influência da aceleração de Coriolis}

A aceleração de Coriolis só aparece no CG da plataforma móvel (ponto $J^{*}$ ) devido ao movimento de alongamento e rotação da cadeia passiva e corresponde à parcela $2 \dot{q_{6}} \dot{\theta}_{5}$ da equação 6.24 do Capítulo 6 :

$$
A_{J}^{n}=2 \dot{q}_{6} \dot{\theta}_{5}+q_{6} \ddot{\theta}_{5}
$$

A segunda parcela desta equação, $q_{6} \ddot{\theta}_{5}$, corresponde ao que chamamos de aceleração tangencial. A aceleração de Coriolis e esta têm a direção normal à cadeia passiva e sua soma nos dá a aceleração total, $A_{J}^{n}$, do ponto $J^{*}$ nesta direção. Podemos então escrever a equação na forma

$$
A_{J}^{n}=A_{c o r}+A_{t g}
$$

Pegando o mecanismo estudado no Exemplo de aplicação 1 para o caso no qual o comprimento da cadeia passiva é igual a $0,4 m$ e variando as componentes da velocidade e da aceleração do ponto $J^{*}$ construímos a seguinte tabela 


\begin{tabular}{ccccccccccc}
\hline Sit. & $V_{J x}$ & $V_{J y}$ & $a_{J x}$ & $a_{J y}$ & $\dot{q}_{6}$ & $\dot{\theta}_{5}$ & $A_{c o r}$ & $\ddot{\theta}_{5}$ & $A_{t g}$ & $A_{J}^{n}=A_{c o r}+A_{t g}$ \\
\hline 1 & 1 & 0 & 0 & 0 & 0 & $-2,5$ & 0 & 0 & 0 & 0 \\
2 & 0 & 1 & 0 & 0 & 1 & 0 & 0 & 0 & 0 & 0 \\
3 & 0 & 0 & 1 & 0 & 0 & 0 & 0 & $-2,5$ & -1 & -1 \\
4 & 0 & 0 & 0 & 1 & 0 & 0 & 0 & 0 & 0 & 0 \\
5 & 0 & 0 & 1 & 1 & 0 & 0 & 0 & $-2,5$ & -1 & -1 \\
6 & 1 & 0 & 1 & 0 & 0 & $-2,5$ & 0 & $-2,5$ & -1 & -1 \\
7 & 0 & 1 & 0 & 1 & 1 & 0 & 0 & 0 & 0 & 0 \\
8 & 1 & 0 & 1 & 1 & 0 & $-2,5$ & 0 & $-2,5$ & -1 & -1 \\
9 & 0 & 1 & 1 & 1 & 1 & 0 & 0 & $-2,5$ & -1 & -1 \\
10 & 1 & 1 & 0 & 0 & 1 & $-2,5$ & -5 & 12,5 & 5 & 0 \\
11 & 1 & 1 & 1 & 0 & 1 & $-2,5$ & -5 & 10 & 4 & -1 \\
12 & 1 & 1 & 0 & 1 & 1 & $-2,5$ & -5 & 12,5 & 5 & 0 \\
13 & 1 & 1 & 1 & 1 & 1 & $-2,5$ & -5 & 10 & 4 & -1 \\
\hline
\end{tabular}

Tabela 10.5: Acelerações tangenciais e de Coriolis

Conforme a tabela acima somente nas situações 10 a 13 houve influência da aceleração de Coriolis. Ou seja, somente nos casos em que o ponto $J^{*}$ está sujeito a velocidade constante ou variável cuja direção não seja nem coincidente e nem perpendicular à direção da cadeia passiva, existirá aceleração de Coriolis. Mas qual é o peso desta influência no torque dos atuadores?

Vamos então fazer as tabelas seguintes para as situações 10 a 13 mostrando os torques nos atuadores, considerando em primeiro lugar o efeito da aceleração de Coriolis e em segundo lugar vamos eliminar o efeito desta aceleração.

\begin{tabular}{lllll}
\hline Sit. & 10 & 11 & 12 & 13 \\
\hline$T_{1 D} \operatorname{com} A_{\text {cor }}(N m)$ & $-8,09$ & $-8,90$ & $-8,93$ & $-9,75$ \\
$T_{1 D}$ sem $A_{\text {cor }}(N m)$ & $-7,32$ & $-8,13$ & $-8,16$ & $-8,98$ \\
$|\Delta|(N m)$ & 0,77 & 0,77 & 0,77 & 0,77 \\
$\%$ & 10,52 & 9,47 & 9,44 & 8,57 \\
\hline
\end{tabular}

Tabela 10.6: Influência da aceleração de Coriolis em T1D 


\begin{tabular}{lllll}
\hline Sit. & 10 & 11 & 12 & 13 \\
\hline$T_{1} \operatorname{com} A_{\text {cor }}(N m)$ & 12,78 & 11,97 & 13,63 & 12,81 \\
$T_{1} \operatorname{sem} A_{\text {cor }}(N m)$ & 13,55 & 12,74 & 14,40 & 13,58 \\
$|\Delta|(N m)$ & 0,77 & 0,77 & 0,77 & 0,77 \\
$\%$ & 5,68 & 6,04 & 5,35 & 5,67 \\
\hline
\end{tabular}

Tabela 10.7: Influência da aceleração de Coriolis em T1

Consequentemente o incremento do torque nos atuadores devido à aceleração de Coriolis é sempre igual, pois esta não variou para os quatro casos e porcentualmente vemos que sua influência não é desprezível.

\subsection{Efeito do atrito nas juntas}

Neste estudo consideraremos somente o efeito do atrito na junta prismática porque nos rolamentos radiais o coeficiente de atrito é aproximadamente 0,001, enquanto que numa guia linear leve como a tipo LHK20AN do fabricante NSK e que tem somente $20 \mathrm{~mm}$ de trilho e $69,8 \mathrm{~mm}$ de comprimento, o seu coeficiente de atrito é de 0,004 e a força de atrito de sua proteção contra pó é de aproximadamente $6 \mathrm{~N}$. Lògicamente haverá situações nas quais a influência do atrito nas juntas de rotação, devido à sua quantidade que é 7 e principalmente se for tipo bucha em vez de rolamento, poderá ser maior que a da guia linear. Outra particularização do nosso trabalho é que só abordaremos o atrito de Coulomb.

Nos exemplos de aplicação que fizemos no capítulo anterior adotamos uma guia linear sem esferas, isto é, construída de aço e bronze e, portanto, com coeficiente de atrito igual a 0,2 e força de atrito da proteção igual a $5 \mathrm{~N}$. Vamos então discutir estes exemplos.

No Exemplo de aplicação 1 o mecanismo tem uma posição simétrica em relação ao seu eixo vertical central (cadeia passiva perpendicular à base) e variamos somente o comprimento da cadeia passiva. Desta forma, para cada comprimento da cadeia passiva, os torques nos atuadores têm o mesmo módulo e não existe torque na junta prismática. Portanto o incremento do torque nos atuadores devido à influência do atrito será causado somente pela força de atrito da proteção contra pó da guia linear. Pela tabela seguinte vemos que à medida que a cadeia passiva aumenta diminui o incremento do torque devido ao atrito. 


\begin{tabular}{ccccc}
\hline$q_{6}(m)$ & 0,4 & 0,5 & 0,6 & 0,7 \\
\hline$T_{1}$ com atrito $(N m)$ & 12,25 & 11,15 & 9,69 & 8,05 \\
$T_{1}$ sem atrito $(N m)$ & 10,52 & 9,58 & 8,37 & 7,09 \\
$\Delta(N m)$ & 1,73 & 1,57 & 1,32 & 0,96 \\
$\%$ & 16,44 & 16,38 & 15,77 & 13,54 \\
\hline
\end{tabular}

Tabela 10.8: Exemplo de aplicação 1 sem e com atrito

Nesta tabela não consideramos o efeito de forças na garra, mas tão somente os pesos dos elos do mecanismo. Se considerarmos agora a aplicação de uma força vertical de $100 \mathrm{~N}$ na garra, o incremento do torque nos atuadores, devido ao atrito, para a posição $q_{6}=0,4 \mathrm{~m}$ será de $(46,89-45,16)=1,73 N \mathrm{~m}$. Ou seja, o incremento do torque devido ao atrito não varia com o aumento da força na garra para um dado comprimento da cadeia passiva. Isto é compreensível, pois como não há torque na guia linear o incremento devido ao atrito se refere somente à força de atrito da proteção de pó, que é uma força constante. Evidentemente quando alteramos o comprimento da cadeia passiva este incremento variará, pois devido às novas posições dos elos os torques nos atuadores modificarão.

No Exemplo de aplicação 2 mantivemos o comprimento da cadeia passiva e variamos sua inclinação. Quando a cadeia passiva sai de sua posição vertical começa a surgir torque, e consequentemente força de atrito, na guia linear, os quais vão se aumentando com a inclinação da cadeia passiva em relação à vertical, conforme mostra a tabela seguinte:

\begin{tabular}{cccccc}
\hline$\theta_{5}$ & $90^{\circ}$ & $80^{\circ}$ & $70^{\circ}$ & $60^{\circ}$ & $50^{\circ}$ \\
\hline Torque na guia linear: $T_{4}(N m)$ & 0 & 0,14 & 0,35 & 0,67 & 1,16 \\
Força de atrito na guia linear: $F_{a}(N)$ & 5 & 5,41 & 6,0 & 6,94 & 8,30 \\
\hline
\end{tabular}

Tabela 10.9: Torque e atrito na guia linear

Portanto o incremento $|\Delta|$ do torque em ambos os atuadores também aumenta à medida que aumenta a inclinação da cadeia passiva, conforme vemos nas tabelas seguintes: 


\begin{tabular}{ccccc}
\hline$\theta_{5}$ & $80^{\circ}$ & $70^{\circ}$ & $60^{\circ}$ & $50^{\circ}$ \\
\hline$T_{1 D}$ com atrito $(N m)$ & $-10,26$ & $-8,93$ & $-7,27$ & $-5,40$ \\
$T_{1 D}$ sem atrito $(N m)$ & $-8,53$ & $-7,01$ & $-5,11$ & $-2,96$ \\
$|\Delta|(N m)$ & 1,73 & 1,92 & 2,16 & 2,44 \\
$\%$ & 20,28 & 27,39 & 42,27 & 82,24 \\
\hline
\end{tabular}

Tabela 10.10: Influência do atrito em T1D

\begin{tabular}{ccccc}
\hline$\theta_{5}$ & $80^{\circ}$ & $70^{\circ}$ & $60^{\circ}$ & $50^{\circ}$ \\
\hline$T_{1}$ com atrito & 11,73 & 11,78 & 11,40 & 10,74 \\
$T_{1}$ sem atrito & 10,13 & 10,15 & 9,71 & 8,97 \\
$|\Delta|$ & 1,60 & 1,63 & 1,69 & 1,77 \\
$\%$ & 15,79 & 16,06 & 17,4 & 19,73 \\
\hline
\end{tabular}

Tabela 10.11: Influência do atrito em T1

Nas tabelas acima consideramos somente o peso dos elos. Não houve ação de força na garra. Nas próximas tabelas incluiremos uma força vertical de $100 \mathrm{~N}$ na garra.

\begin{tabular}{ccc}
\hline$\theta_{5}$ & $80^{\circ}$ & $50^{\circ}$ \\
\hline$T_{1 D}$ com atrito $(N m)$ & $-39,12$ & $-21,16$ \\
$T_{1 D}$ sem atrito $(N m)$ & $-36,99$ & $-15,34$ \\
$\Delta(N m)$ & $-2,13$ & $-5,82$ \\
$\%$ & 5,75 & 37,94 \\
\hline
\end{tabular}

Tabela 10.12: Influência do atrito com carga externa em T1

\begin{tabular}{ccc}
\hline$\theta_{5}$ & $80^{\circ}$ & $50^{\circ}$ \\
\hline$T_{1}$ com atrito $(N m)$ & 44,54 & 42,40 \\
$T_{1}$ sem atrito $(N m)$ & 42,59 & 38,05 \\
$\Delta(N m)$ & 1,95 & 4,35 \\
$\%$ & 4,58 & 11,43 \\
\hline
\end{tabular}

Tabela 10.13: Influência do atrito com carga externa em T1D

Pelas tabelas acima podemos concluir que, aumentando-se a carga na garra o porcentual do incremento do torque nos atuadores, devido ao atrito na guia linear, diminui. Ou seja, a influência da força de atrito no torque dos atuadores não aumenta na mesma razão do aumento da força externa atuante na garra. E 
isto é explicável, pois na força de atrito existe uma parcela fixa referente ao atrito da proteção contra pó. Logo, a sua razão de crescimento é menor que a razão de crescimento da força externa e, consequentemente, dos torques nos atuadores. 


\section{Capítulo 11: CONCLUSÕES}

Nesta dissertação, apresentamos um robô paralelo, concebido com a finalidade de realizar operações "pega-e-põe"no espaço bidimensional (plano).

De modo a caracterizar, tanto cinematicamente quanto dinamicamente, o mecanismo selecionado para o robô, desenvolvemos sua correspondente modelagem matemática da forma mais abrangente possível.

Com relação à cinemática, realizamos as análises de posições, velocidades e acelerações, a investigação de ocorrência de singularidades, o levantamento do espaço de trabalho disponível, bem como a avaliação dos movimentos da garra do robô, segundo dois tipos possíveis de trajetórias, retilínea e circular.

Com relação à estática efetuamos sua análise, realizando inclusive o balanceamento do mecanismo com a adição de contrapesos.

Com relação à dinâmica, obtivemos as expressões explícitas dos torques dos atuadores empregando o método de Kane. Efetuamos a análise considerando também o efeito do atrito de Coulomb.

Observamos algumas vantagens na formulação de Kane sobre os métodos clássicos como o de Newton-Euler e de Lagrange.

Como temas para futuros trabalhos podemos sugerir:

- Adoção de técnicas de balanceamento sem a adição de massa, utilizando molas.

- Consideração do efeito do atrito viscoso na junta prismática bem como nas juntas de rotação.

- Comparação de maior profundidade entre os métodos de Kane e Lagrange.

- Simular o comportamento do robô para uma trajetória qualquer.

- Estender o desenvolvimento realizado no espaço bidimensional para o tridimensional. 


\section{Anexo A: PROGRAMA CINEMÁtICA INVERSA}

Neste anexo apresentamos o programa do software Mathematica para solução da cinemática inversa e verificação de singularidades.

Fornecendo-se os seguintes dados:

- Comprimento dos elos: $L_{1}$ a $L_{6}$.

- Comprimento da cadeia passiva e seu ângulo de inclinação: $L_{7}$ e $\theta_{7}$.

O programa determina:

- Os ângulos de entrada: $\theta_{2}$ e $\theta_{6}$.

- Os ângulos dos elos $L_{3}$ e $L_{5}: \theta_{3}$ e $\theta_{5}$.

- Verifica a existência de singularidades e informa seus tipos.

- Traça o gráfico do mecanismo

No programa impomos as condições de $A$ estar sempre à esquerda do segmento $\mathrm{O}_{2} \mathrm{~B}$ e $\mathrm{D}$ à direita de $\mathrm{O}_{6} \mathrm{C}$.

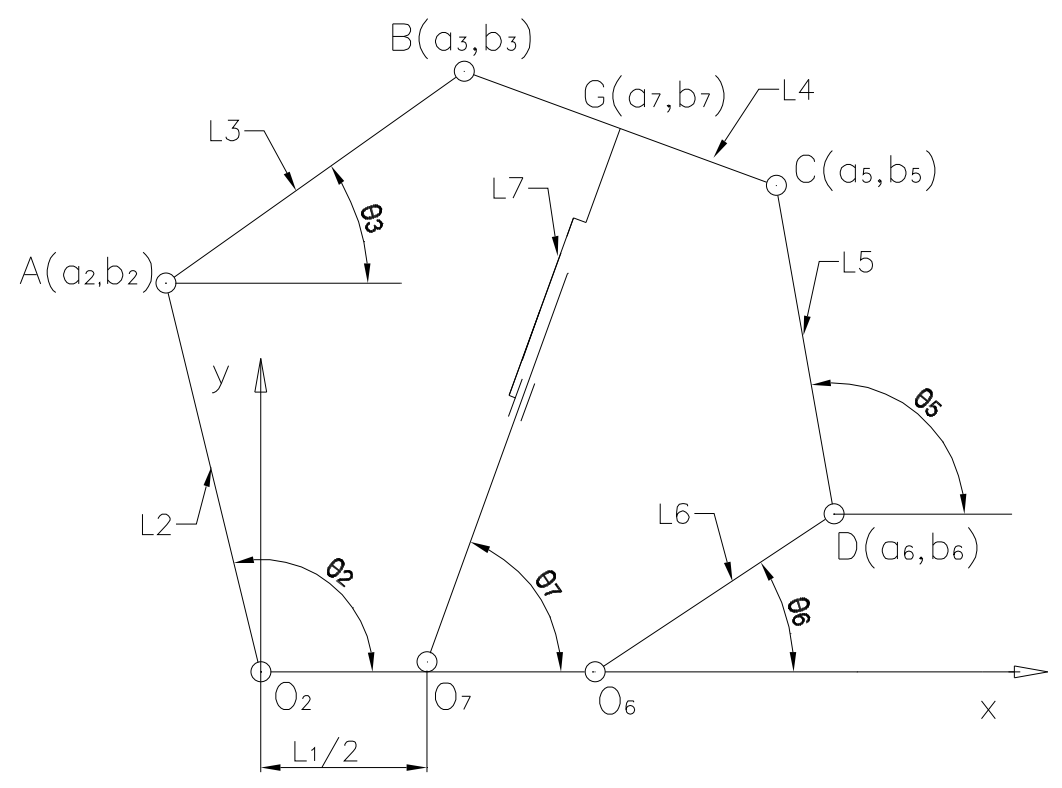

Figura 11.1: Anexo A: diagrama cinemático 


\section{LISTA DO PROGRAMA}

(* ANEXO A: CINEMATICA INVERSA e det. de singularidades *)

(* Resultado: $\{\mathrm{t} 2, \mathrm{t} 3, \mathrm{t} 5, \mathrm{t} 6$, sing, $\{$ hex, barra $\}$ \} $*$ )

FctA $\left[\mathrm{L} 1_{-}, \mathrm{L} 2_{-}, \mathrm{L} 3_{-}, \mathrm{L} 4_{-}, \mathrm{L} 5_{-}, \mathrm{L} 6_{-}, \mathrm{L} 7_{-}, \mathrm{t} 77_{-}\right]:=$

Module $[\{a 7, b 7, a 5, b 5, a 3, b 3$, sol6, sol2, $\mathrm{a} 61, \mathrm{~b} 61, \mathrm{a} 62$, b62, a21, b21, a22, b22, fsol, sing, $\mathrm{s} 7, \mathrm{c7}, \mathrm{s} 2, \mathrm{c} 2, \mathrm{~s} 6, \mathrm{c} 6\}$,

$s 7=\operatorname{Sin}[t 7] ; c 7=\operatorname{Cos}[t 7] ;$

(* Coordenadas de $\mathrm{G} *$ )

$\{\mathrm{a} 7, \mathrm{~b} 7\}=\{\mathrm{L} 1 / 2+\mathrm{L} 7 * \mathrm{c} 7, \mathrm{~L} 7 * \mathrm{~s} 7\}$;

(* Coordenadas de $\mathrm{B} *$ )

$\{\mathrm{a} 3, \mathrm{~b} 3\}=\mathrm{L} 4 / 2 *\{-\mathrm{s} 7, \mathrm{c} 7\}+\{\mathrm{a} 7, \mathrm{~b} 7\}$;

(* Coordenadas de $\mathrm{C} *$ )

$\{\mathrm{a} 5, \mathrm{~b} 5\}=\mathrm{L} 4 / 2 *\{\mathrm{~s} 7,-\mathrm{c} 7\}+\{\mathrm{a} 7, \mathrm{~b} 7\}$;

(* Coordenadas de $\mathrm{A} *$ )

sol2=Solve $\left[\left\{x^{\wedge} 2+y^{\wedge} 2==L 2 \wedge 2,(x-a 3)^{\wedge} 2+(y-b 3)^{\wedge} 2==L 3^{\wedge} 2\right\},\{x, y\}\right]$;

$\{\mathrm{a} 21, \mathrm{~b} 21\}=\operatorname{ReplaceAll}[\{\mathrm{x}, \mathrm{y}\}$, sol2 $[[1]]]$;

$\{\mathrm{a} 22, \mathrm{~b} 22\}=\operatorname{ReplaceAll}[\{\mathrm{x}, \mathrm{y}\}, \mathrm{sol} 2[[2]]]$;

(* Coordenadas de $\mathrm{D} *$ )

sol6=Solve $\left[\left\{(\mathrm{x}-\mathrm{L} 1)^{\wedge} 2+\mathrm{y}^{\wedge} 2==\mathrm{L} 6^{\wedge} 2,(\mathrm{x}-\mathrm{a} 5)^{\wedge} 2+(\mathrm{y}-\mathrm{b} 5)^{\wedge} 2==\mathrm{L} 5^{\wedge} 2\right\},\{\mathrm{x}, \mathrm{y}\}\right]$;

$\{\mathrm{a} 61, \mathrm{~b} 61\}=\operatorname{ReplaceAll}[\{\mathrm{x}, \mathrm{y}\}$, sol6 $[[1]]]$;

$\{\mathrm{a} 62, \mathrm{~b} 62\}=\operatorname{ReplaceAll}[\{\mathrm{x}, \mathrm{y}\}$, sol6 $[[2]]]$;

(* Achar os pontos A e D que satisfazem a condicao de estarem sempre para fora $*$ )

$$
\begin{aligned}
\operatorname{If}[((\mathrm{a} 61-\mathrm{a} 62) * \mathrm{~b} 5-(\mathrm{b} 61-\mathrm{b} 62) *(\mathrm{a} 5-\mathrm{L} 1))> & =0 \& \&((\mathrm{a} 21-\mathrm{a} 22) * \\
\mathrm{b} 3-(\mathrm{b} 21-\mathrm{b} 22) * \mathrm{a} 3)>=0, \mathrm{fsol} & =\{\{\mathrm{a} 61, \mathrm{~b} 61\},\{\mathrm{a} 22, \mathrm{~b} 22\}\},
\end{aligned}
$$




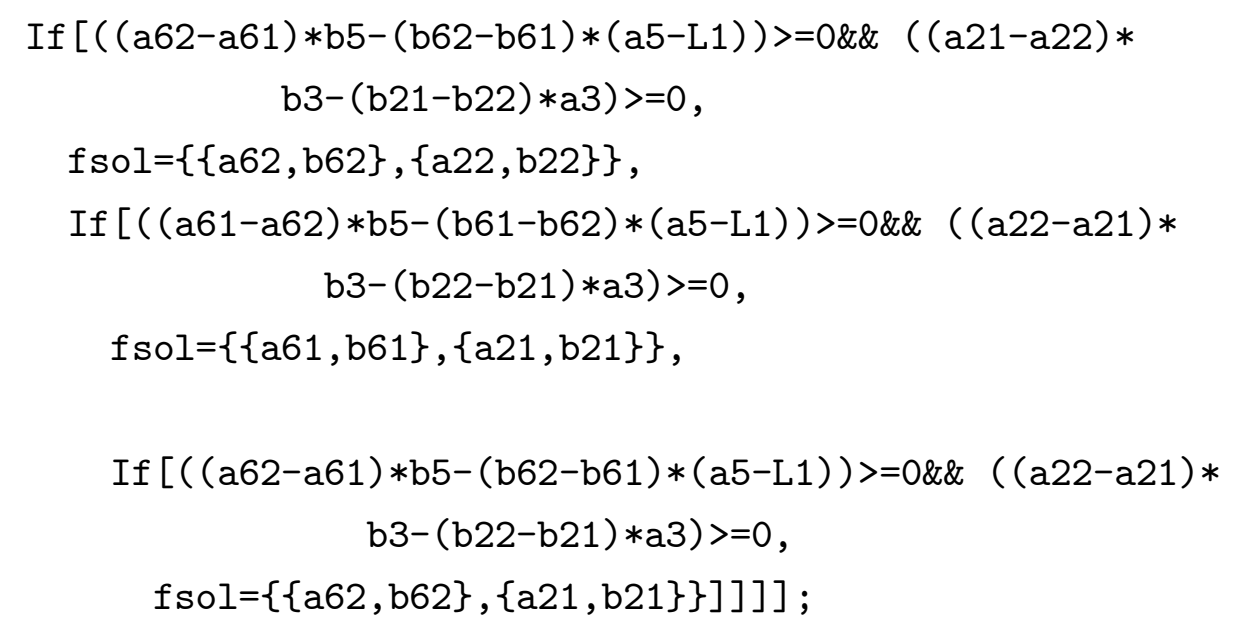

$\{\mathrm{a} 6, \mathrm{~b} 6\}=\mathrm{f}$ sol $[[1]]$;

$\{\mathrm{a} 2, \mathrm{~b} 2\}=\mathrm{fsol}[[2]]$;

(* Calculo dos angulos *)

If $[\mathrm{b} 2>=0, \mathrm{t} 2=\mathrm{ArcCos}[\mathrm{a} 2 / \mathrm{L} 2], \mathrm{t} 2=2 * \mathrm{Pi}-\operatorname{ArcCos}[\mathrm{a} 2 / \mathrm{L} 2]]$;

If $[\mathrm{b} 3>=\mathrm{b} 2, \mathrm{t} 3=\operatorname{Arc\operatorname {Cos}}[(\mathrm{a} 3-\mathrm{a} 2) / \mathrm{L} 3], \mathrm{t} 3=2 * \mathrm{Pi}-\operatorname{ArcCos}[(\mathrm{a} 3-\mathrm{a} 2) / \mathrm{L} 3]]$;

If $[\mathrm{b} 5>=\mathrm{b} 6, \mathrm{t} 5=\operatorname{ArcCos}[(\mathrm{a} 5-\mathrm{a} 6) / \mathrm{L} 5], \mathrm{t} 5=2 * \mathrm{Pi}-\operatorname{ArcCos}[(\mathrm{a} 5-\mathrm{a} 6) / \mathrm{L} 5]]$;

If $[\mathrm{b} 6>=0, \mathrm{t} 6=\operatorname{ArcCos}[(\mathrm{a} 6-\mathrm{L} 1) / \mathrm{L} 6], \mathrm{t} 6=2 * \mathrm{Pi}-\operatorname{ArcCos}[(\mathrm{a} 6-\mathrm{L} 1) / \mathrm{L} 6]]$;

(* Verificacao das singularidades $*$ )

$\mathrm{s} 2=\operatorname{Sin}[\mathrm{t} 2] ; \mathrm{c} 2=\operatorname{Cos}[\mathrm{t} 2] ; \mathrm{s} 6=\mathrm{Sin}[\mathrm{t} 6] ; \mathrm{c} 6=\operatorname{Cos}[\mathrm{t} 6]$;

$\mathrm{p} 11=-2 * \mathrm{~L} 2 * \mathrm{c} 2 * \mathrm{c} 7+\mathrm{L} 1 * \mathrm{c} 7-2 * \mathrm{~L} 2 * \mathrm{~s} 2 * \mathrm{~s} 7+2 * \mathrm{~L} 7$

$\mathrm{p} 12=2 * \mathrm{~L} 2 * \mathrm{~L} 7 * \mathrm{c} 2 * \mathrm{~s} 7+\mathrm{L} 2 * \mathrm{~L} 4 * \mathrm{~s} 2 * \mathrm{~s} 7-\mathrm{L} 1 * \mathrm{~L} 7 * \mathrm{~s} 7+\mathrm{L} 4 * \mathrm{~L} 2 * \mathrm{c} 2 * \mathrm{c} 7-$

$2 * \mathrm{~L} 2 * \mathrm{~L} 7 * \mathrm{~s} 2 * \mathrm{c} 7-\mathrm{L} 1 * \mathrm{~L} 4 * \mathrm{c} 7 / 2$;

$\mathrm{q} 11=2 * \mathrm{~L} 2 * \mathrm{~L} 7 * \mathrm{~s} 2 * \mathrm{c} 7-\mathrm{L} 2 * \mathrm{~L} 4 * \mathrm{c} 2 * \mathrm{c} 7-\mathrm{L} 4 * \mathrm{~L} 2 * \mathrm{~s} 2 * \mathrm{~s} 7-2 * \mathrm{~L} 2 * \mathrm{~L} 7 * \mathrm{c} 2 * \mathrm{~s} 7-$ $\mathrm{L} 2 * \mathrm{~L} 1 * \mathrm{~s} 2$;

$\mathrm{p} 21=-2 * \mathrm{~L} 6 * \mathrm{c} 6 * \mathrm{c} 7-\mathrm{L} 1 * \mathrm{c} 7-2 * \mathrm{~L} 6 * \mathrm{~s} 6 * \mathrm{~s} 7+2 * \mathrm{~L} 7$;

p22 $=2 * \mathrm{~L} 6 * \mathrm{~L} 7 * \mathrm{c} 6 * \mathrm{~s} 7-\mathrm{L} 6 * \mathrm{~L} 4 * \mathrm{~s} 6 * \mathrm{~s} 7+\mathrm{L} 1 * \mathrm{~L} 7 * \mathrm{~s} 7-\mathrm{L} 4 * \mathrm{~L} 6 * \mathrm{c} 6 * \mathrm{c} 7-$

$2 * \mathrm{~L} 6 * \mathrm{~L} 7 * \mathrm{~s} 6 * \mathrm{c} 7-\mathrm{L} 1 * \mathrm{~L} 4 * \mathrm{c} 7 / 2$;

$\mathrm{q} 22=2 * \mathrm{~L} 6 * \mathrm{~L} 7 * \mathrm{~s} 6 * \mathrm{c} 7+\mathrm{L} 6 * \mathrm{~L} 4 * \mathrm{c} 6 * \mathrm{c} 7+\mathrm{L} 4 * \mathrm{~L} 6 * \mathrm{~s} 6 * \mathrm{~s} 7-2 * \mathrm{~L} 6 * \mathrm{~L} 7 * \mathrm{c} 6 * \mathrm{~s} 7-$ $\mathrm{L} 6 * \mathrm{~L} 1 * \mathrm{~s} 6$;

sing $=0$

$J q=\{\{q 11,0\},\{0, q 22\}\}$ 
If $[\operatorname{Det}[\mathrm{Jq}]==0, \operatorname{sing}=1]$;

$\mathrm{Jx}=\{\{\mathrm{p} 11, \mathrm{p} 12\},\{\mathrm{p} 21, \mathrm{p} 22\}\} ;$

If $[\operatorname{Det}[\mathrm{Jx}]==0$, If $[$ sing $==1$, sing=3, sing=2] ] ;

barra $=$ Graphics $[$ RGBColor $[1,0,0], \operatorname{Line}[\{\{\mathrm{L} 1 / 2,0\},\{\mathrm{a} 7, \mathrm{~b} 7\}\}]\}]$;

vertices $=\{\{0,0\},\{\mathrm{a} 2, \mathrm{~b} 2\},\{\mathrm{a} 3, \mathrm{~b} 3\},\{\mathrm{a} 5, \mathrm{~b} 5\},\{\mathrm{a} 6, \mathrm{~b} 6\},\{\mathrm{L} 1,0\},\{0,0\}\}$;

hex=Graphics [Line [vertices] ] ;

$\operatorname{Return}[\{t 2, t 3, t 5, t 6$, sing, $\{$ hex , barra $\}\}] ;]$ 


\section{Anexo B: PROGRAMA CINEMÁtICA DIRETA}

Neste anexo apresentamos o programa do software Mathematica para solução da cinemática direta.

Fornecendo-se os seguintes dados:

- Comprimento dos elos $L_{1}$ a $L_{6}$

- Ângulos de entrada $\theta_{2}$ e $\theta_{6}$

O programa determina:

- Comprimento da cadeia passiva $L_{7}$

- Ângulo de inclinação $\theta_{7}$

Foram impostas as seguintes condições:

- $L_{7} \geqslant 0$

- $0 \leqslant \theta_{7} \leqslant \pi$

- A plataforma móvel nunca deve cruzar com a base $L_{1}$ ou com os elos inferiores $L_{2}$ e $L_{6}$.

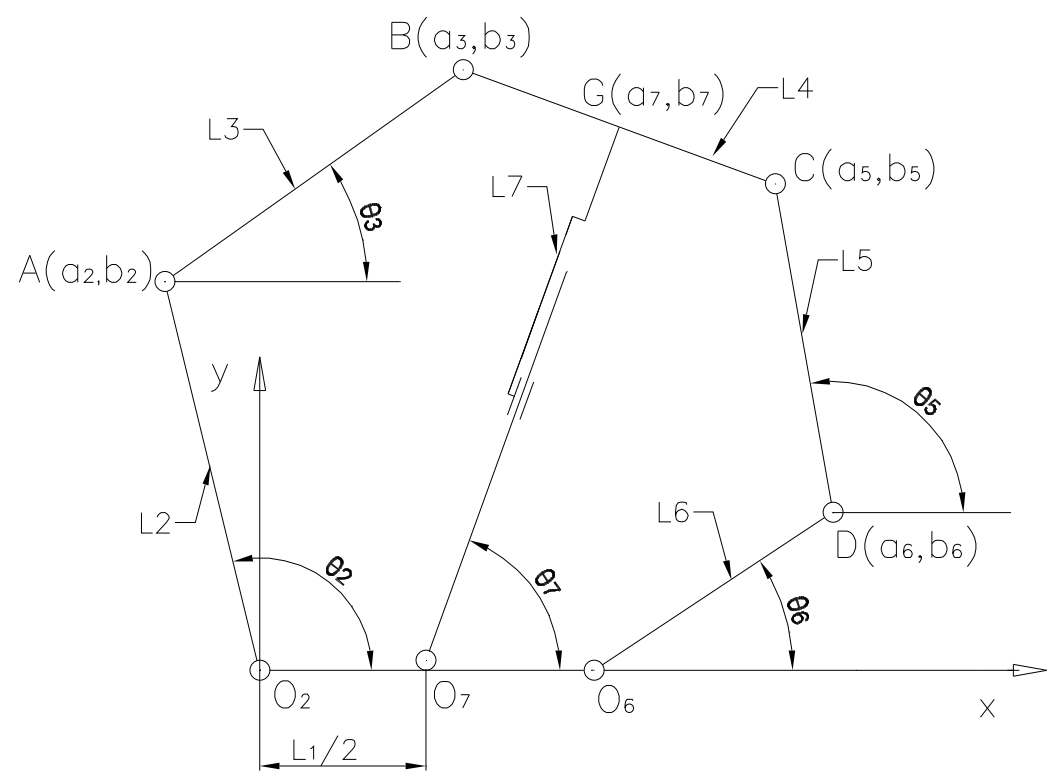

Figura 11.2: Anexo B: diagrama cinemático 
LISTA DO PROGRAMA

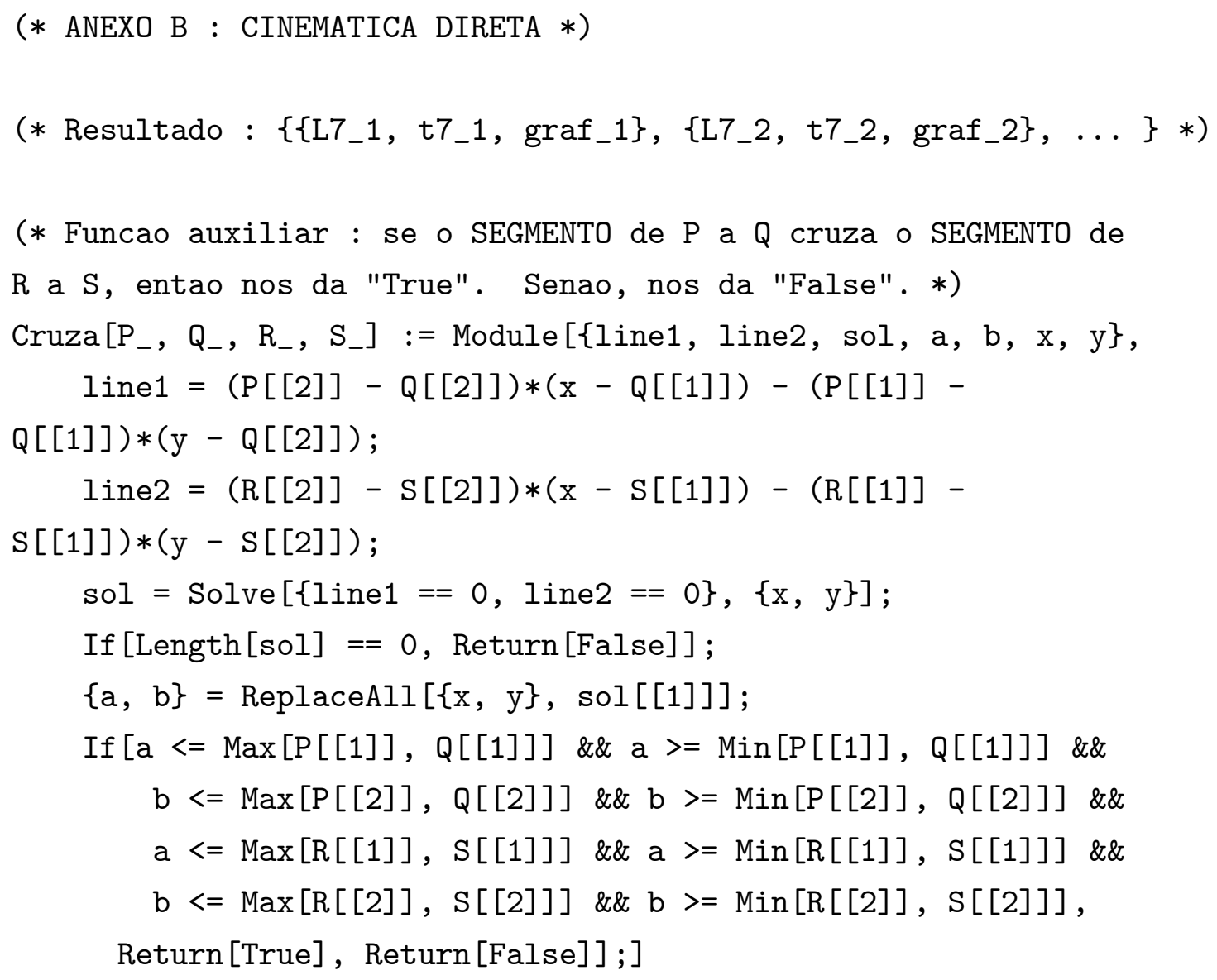




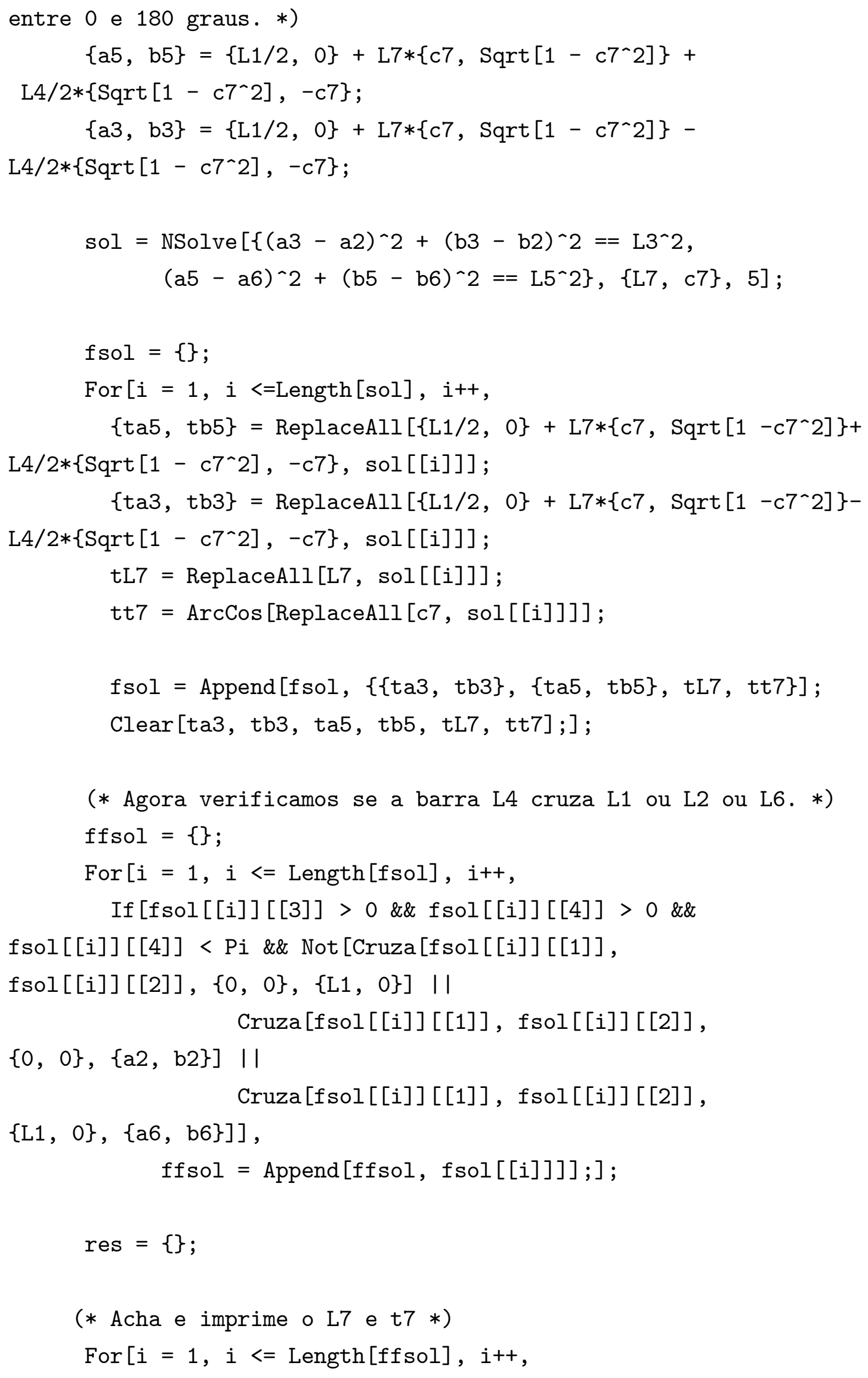




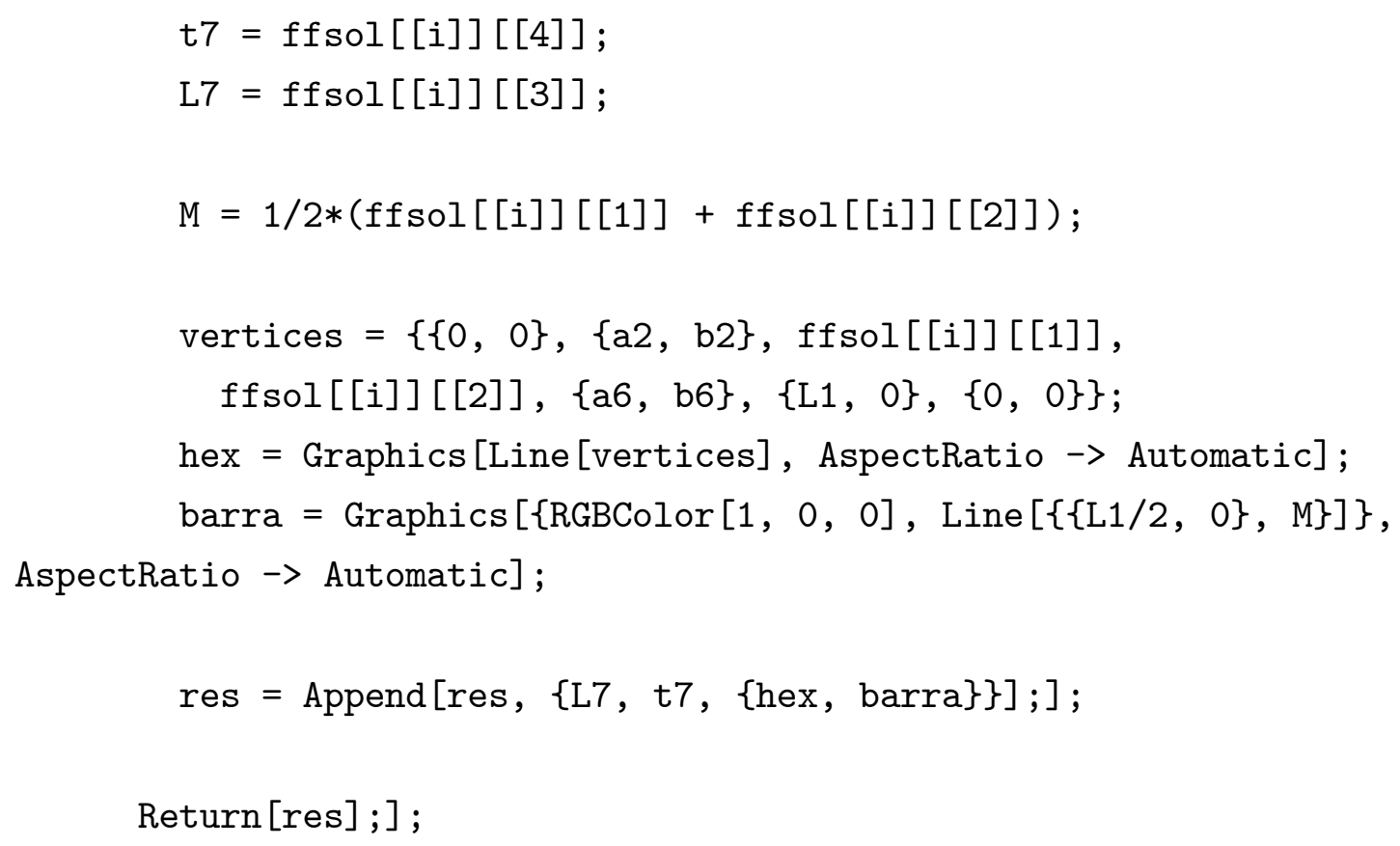




\section{Anexo C: PROGRAMA ESPAÇO DE TRABALHO}

Neste anexo apresentamos o programa executado no software Mathematica que plota o espaço de trabalho do mecanismo.

Os dados a serem fornecidos são os comprimentos dos elos do mecanismo $\left(L_{1}\right.$ a $L_{6}$ ) e o comprimento da cadeia passiva totalmente estendida $(L L)$. Também devemos definir, na listagem do programa, qual deve ser o espaçamento entre pontos nas direções $x$ e $y$.

As seguintes condições são impostas:

$$
\begin{aligned}
0 & \leqslant L_{7} \leqslant L L \\
\left|L_{2}-L_{3}\right| & \leqslant\left\|B-O_{2}\right\| \leqslant L_{3}+L_{2}, \\
\left|L_{6}-L_{5}\right| & \leqslant\left\|C-O_{6}\right\| \leqslant L_{5}+L_{6}
\end{aligned}
$$

\section{LISTA DO PROGRAMA}

(* ANEXO C: ESPACO DE TRABALHO *)

(* Retorna: graf *)

FctC $\left[\mathrm{L} 1_{-}, \mathrm{L} 2_{-}, \mathrm{L} 3_{-}, \mathrm{L} 4_{-}, \mathrm{L}_{-}, \mathrm{L} 66_{-}, \mathrm{LL} L_{-}\right]:=$Module $[$ppontos $, \mathrm{x}, \mathrm{y}, \mathrm{L} 7, \mathrm{t} 7, \mathrm{~s} 7, \mathrm{c} 7\}$ pontos $=\{\}$

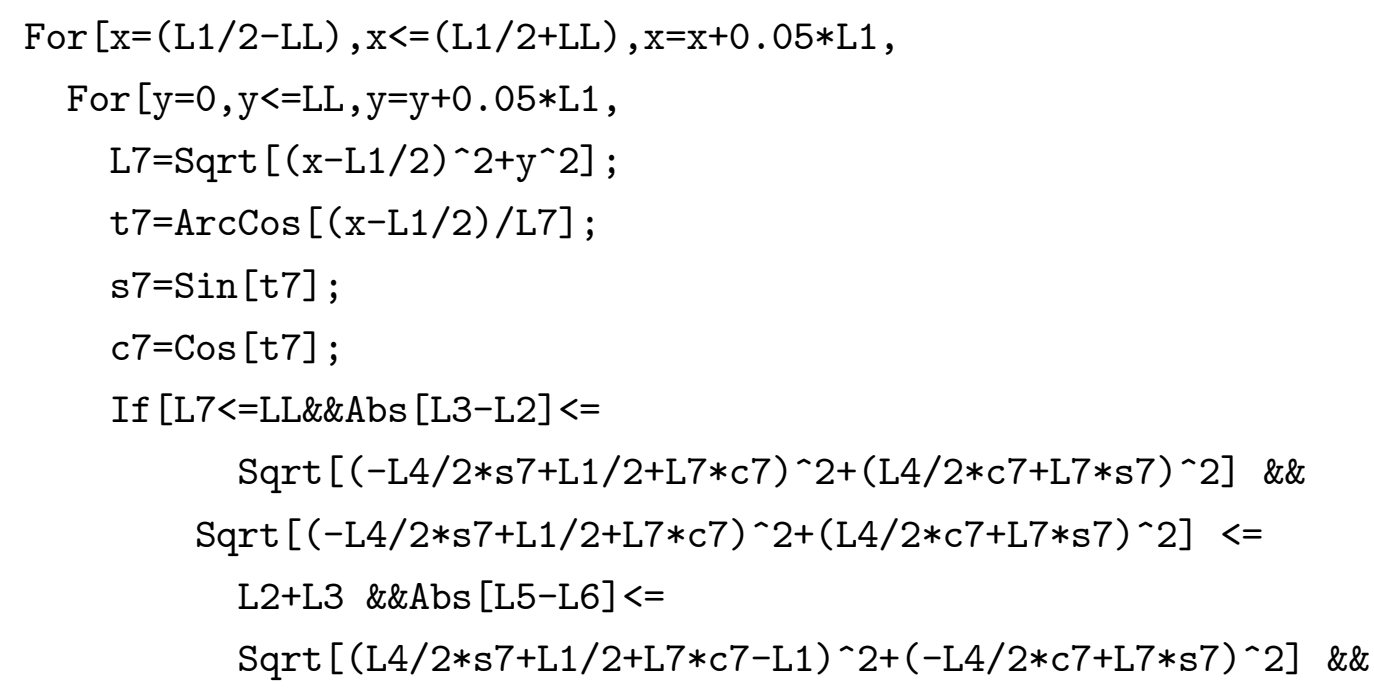


$\operatorname{Sqrt}\left[(\mathrm{L} 4 / 2 * \mathrm{~s} 7+\mathrm{L} 1 / 2+\mathrm{L} 7 * \mathrm{c} 7-\mathrm{L} 1)^{\wedge} 2+(-\mathrm{L} 4 / 2 * \mathrm{c} 7+\mathrm{L} 7 * \mathrm{~s} 7)^{\wedge} 2\right]<=$ L5+L6,

pontos=Append [pontos,$\{\mathrm{x}, \mathrm{y}\}]] ;]]$;

Return [ListPlot [pontos, AxesOrigin-> $\{0,0\}, P$ lotRange $->\{0, L L\}$, AspectRatio->Automatic] ] ; ] 


\section{Anexo D: PROGRAMA DAS VELOCIDADES E ACELERAÇÕES, TRAJETÓRIA RETILÍNEA}

Neste anexo apresentamos o programa desenvolvido no software Mathematica para calcular as componentes da velocidade e da aceleração da garra para qualquer ponto situado numa trajetória linear do mecanismo.

Neste programa consideramos os seguintes dados de projeto:

-Velocidade máxima: $v_{\max }=1,0 \mathrm{~m} / \mathrm{s}$

-Módulo da aceleração igual da desaceleração: $a=10,0 \mathrm{~m} / \mathrm{s}^{2}$

Devemos entrar com os seguintes dados:

-Deslocamento total: $s_{t}$

-Inclinação deste deslocamento em relação ao eixo $x: \varphi$

-Deslocamento do ponto onde queremos achar os resultados: $s$.

O programa mostra os resultados na forma de um vetor: $\left\{v_{x}, v_{y}, a_{x}, a_{y}\right\}$.

LISTA DO PROGRAMA

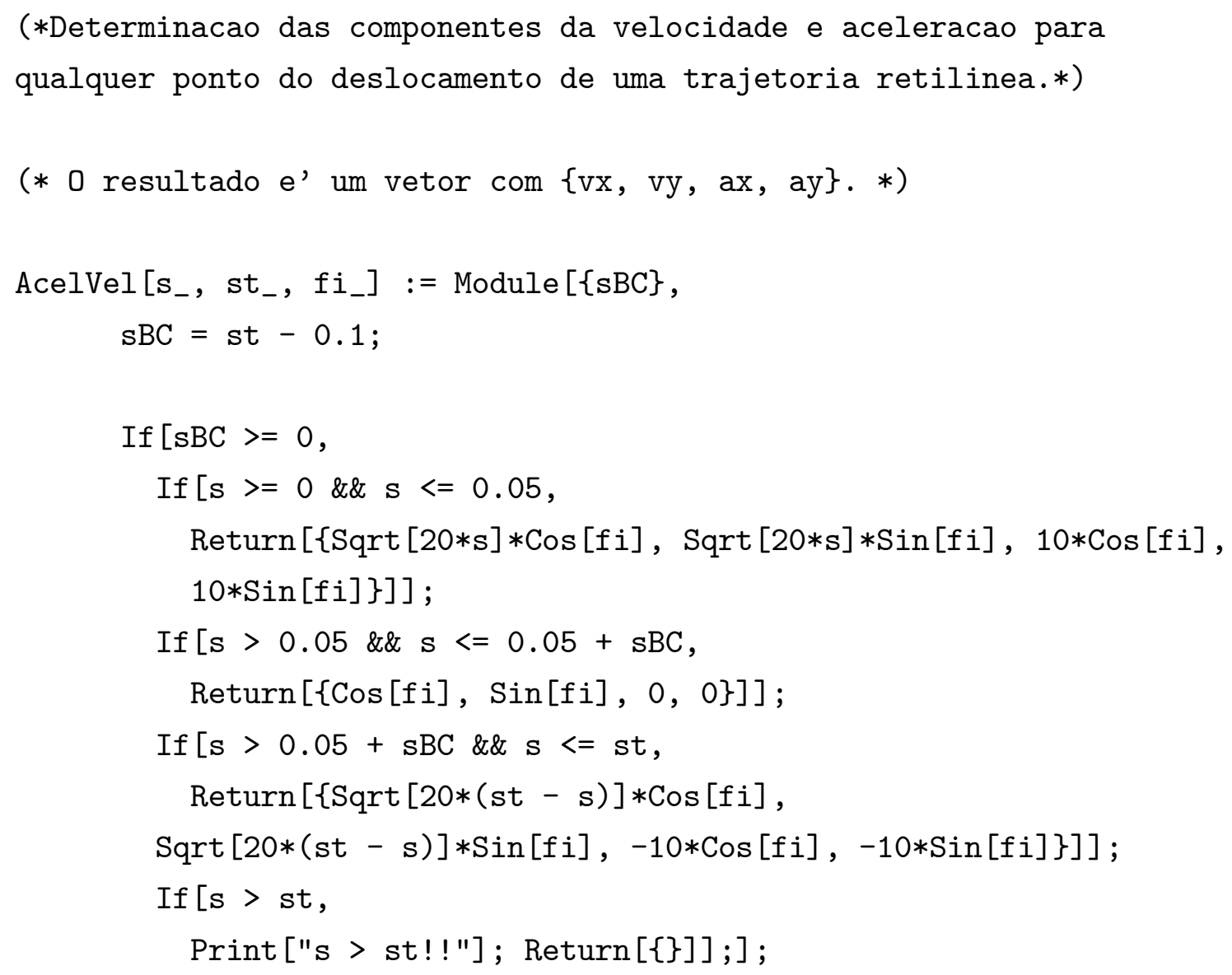




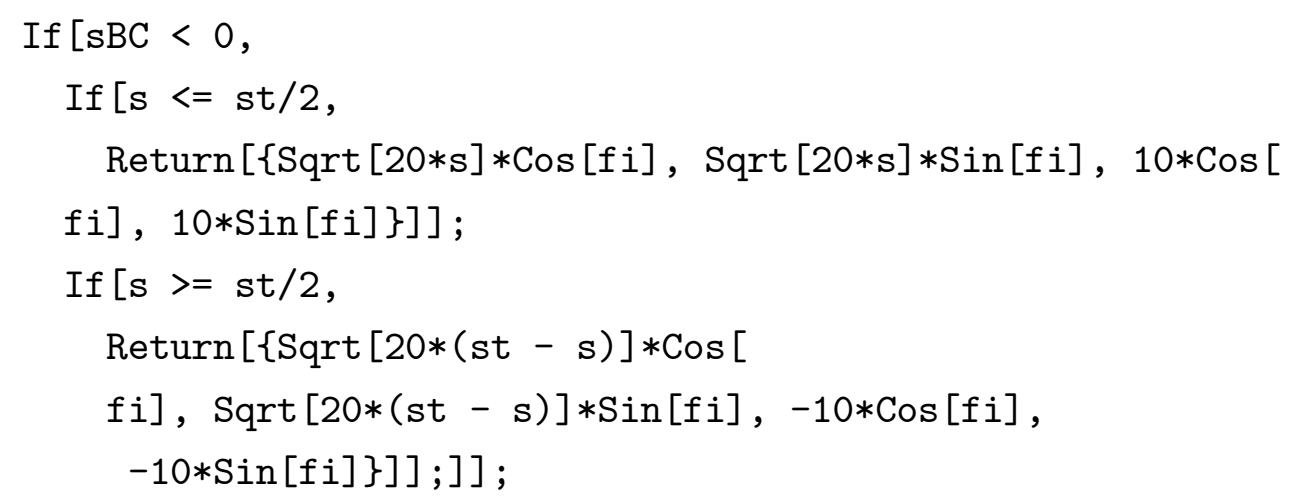




\section{Anexo E: PROGR. VEL. E ACEL., TRAJETÓRIA CIRCULAR E CENTRO NA ORIGEM}

Neste anexo apresentamos o programa desenvolvido no software Mathematica que calcula as componentes da velocidade e da aceleração da garra para qualquer ponto situado numa trajetória circular, cujo centro está na origem dos eixos de coordenadas principal.

Neste programa consideramos os seguintes dados de projeto:

-Velocidade máxima: $v_{\max }=1,0 \mathrm{~m} / \mathrm{s}$

-Módulo da aceleração igual da desaceleração: $10,0 \mathrm{~m} / \mathrm{s}^{2}$

Devemos entrar com os seguintes dados:

-Raio do arco trajetória: $r$

-Aceleração tangencial: $a_{t}=10$

-Ângulo do raio referente ao ponto onde queremos achar os resultados: $\gamma$

-Ângulo do raio referente ao ponto $A$, ponto inicial da trajetória: $\gamma_{A}$

-Ângulo do raio referente ao ponto $D$, ponto final da trajetória: $\gamma_{D}$

-Orientação do arco: sinal =1 (sentido anti-horário), sinal $=-1$ (sentido horário)

O programa mostra os resultados na forma de um vetor: $\left\{\left\{v_{x}, v_{y}\right\},\left\{a_{x}, a_{y}\right\}\right\}$.

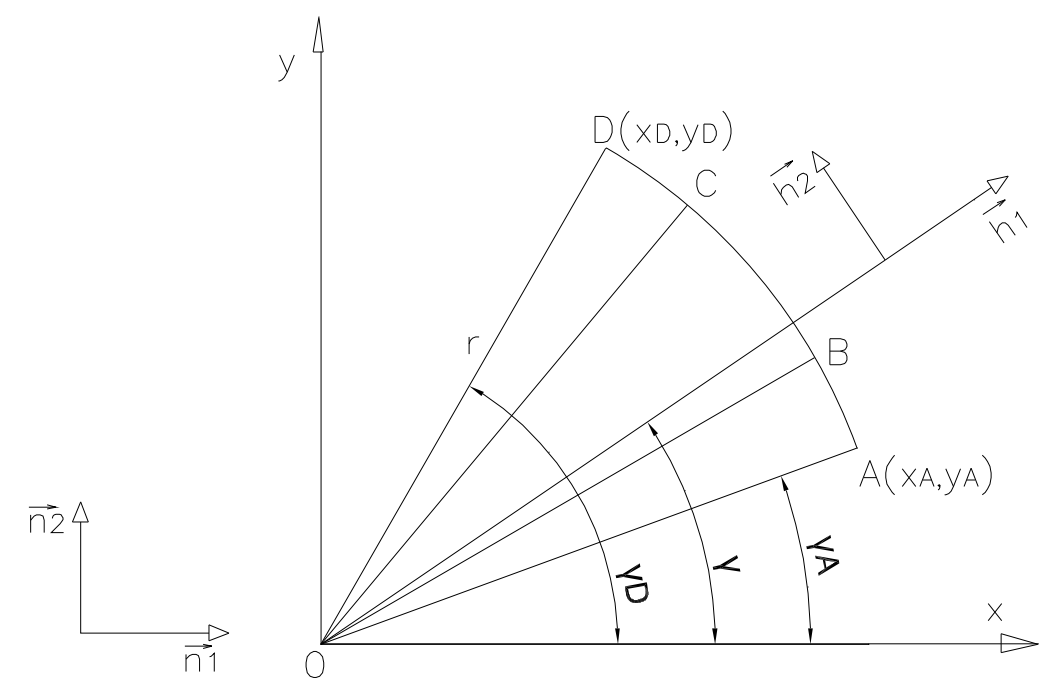

Figura 11.3: Anexo E: trajetória circular com centro na origem

LISTA DO PROGRAMA

(*Determinacao das componentes da velocidade e aceleracao para 
qualquer ponto de uma trajetoria circular, COM CENTRO COINCIDENTE COM A ORIGEM dos eixos de coordenadas.*)

(*Obs. : Todos os angulos devem ser no sentido antihorario a partir do eixo horizontal*)

(* 0 resultado e' um vetor com $\{\{\mathrm{vx}, \mathrm{vy}\},\{\mathrm{ax}, \mathrm{ay}\}\} . *)$

(* Calculo de angulos com a horizontal no sentido anti - horario. 0 e' o centro e A e' um ponto na semireta.*) AngF $\left[0_{-}, A_{-}\right]:=\operatorname{Module}[\{t a, P\}$, $P=(A-0)$; ta $=\operatorname{ArcCos}[\mathrm{P}[[1]] / \operatorname{Norm}[\mathrm{P}]]$; If $[\mathrm{P}[[2]]>=0, \operatorname{Return}[\mathrm{ta}], \operatorname{Return}[2 * \mathrm{Pi}-\mathrm{ta}]] ;]$

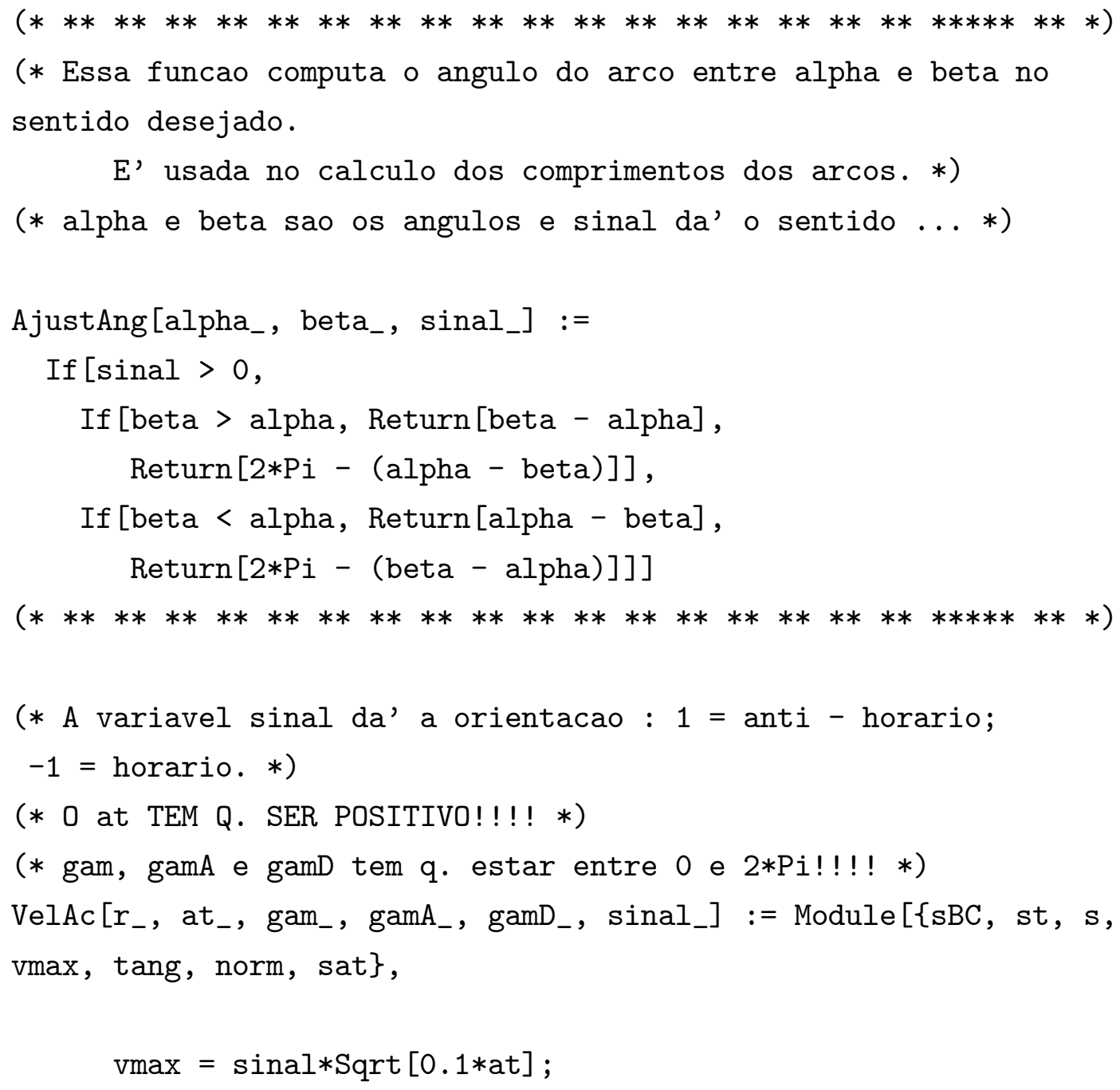




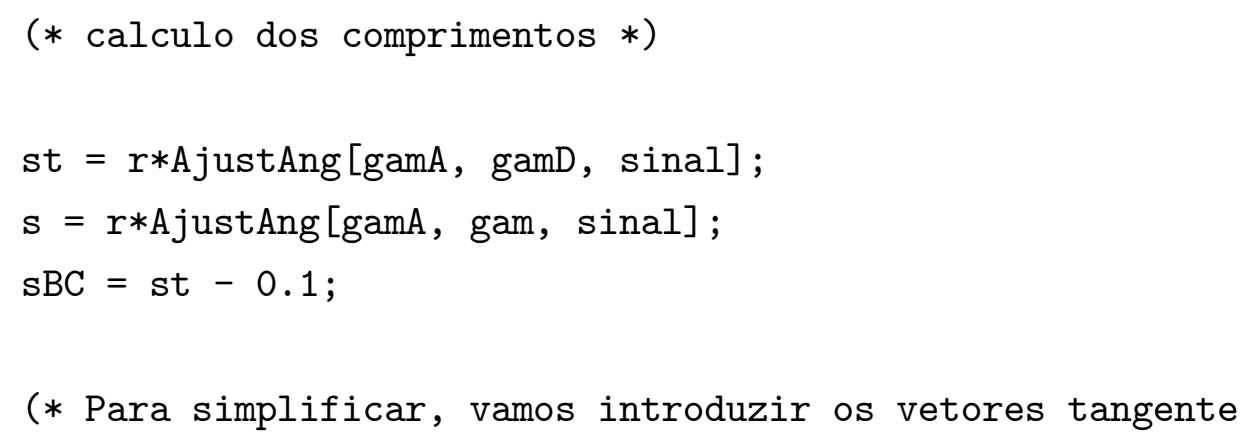
e normal. *)

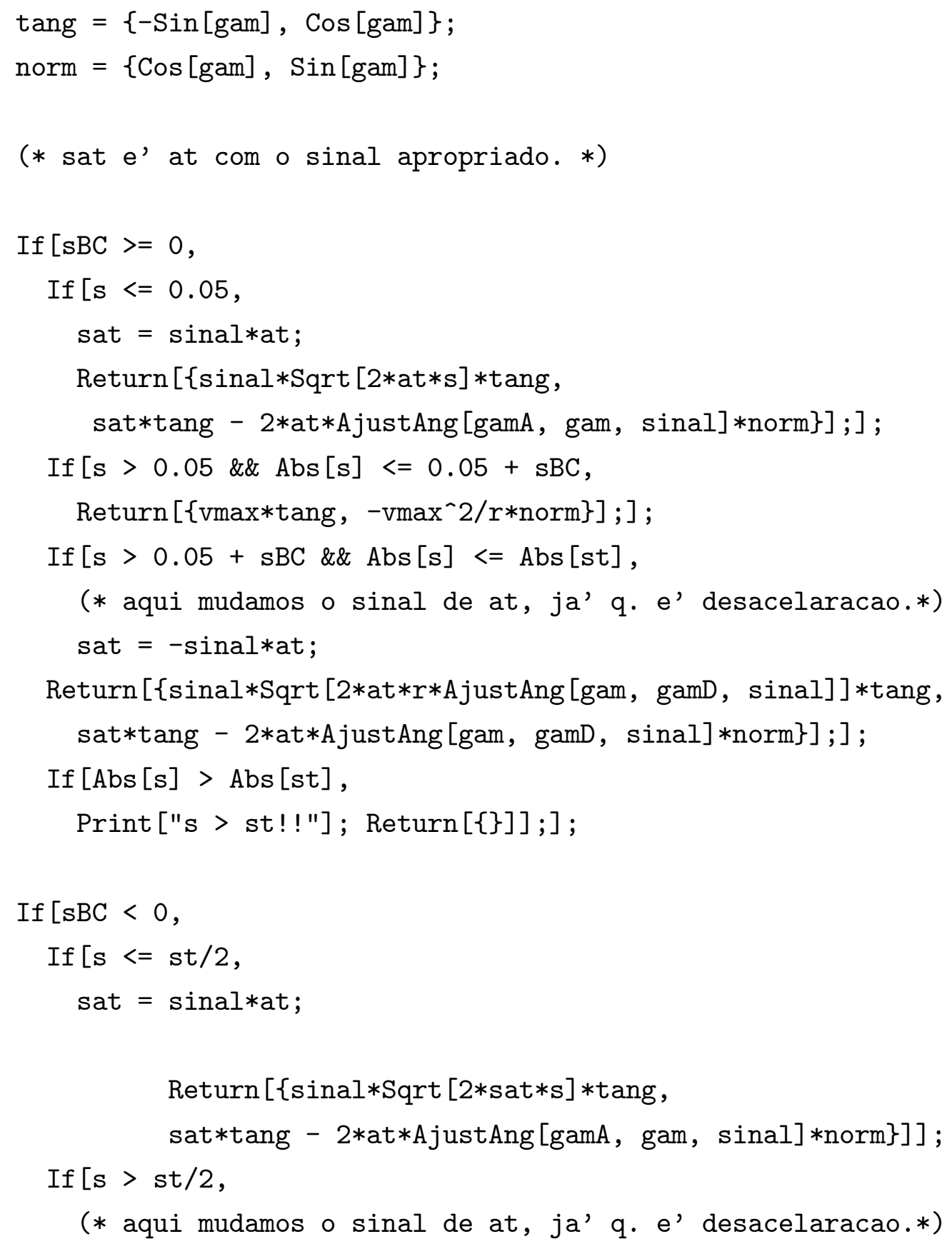




$$
\text { sat }=- \text { sinal } * \text { at }
$$

$\operatorname{Return}[\{\operatorname{sinal} * \operatorname{Sqrt}[2 * \operatorname{at} * r * A j u s t A n g[\mathrm{gam}, \operatorname{gamD}$, sinal $]] * \operatorname{tang}$, sat*tang - 2*at*AjustAng[gam, gamD, sinal]*norm\}]];]];

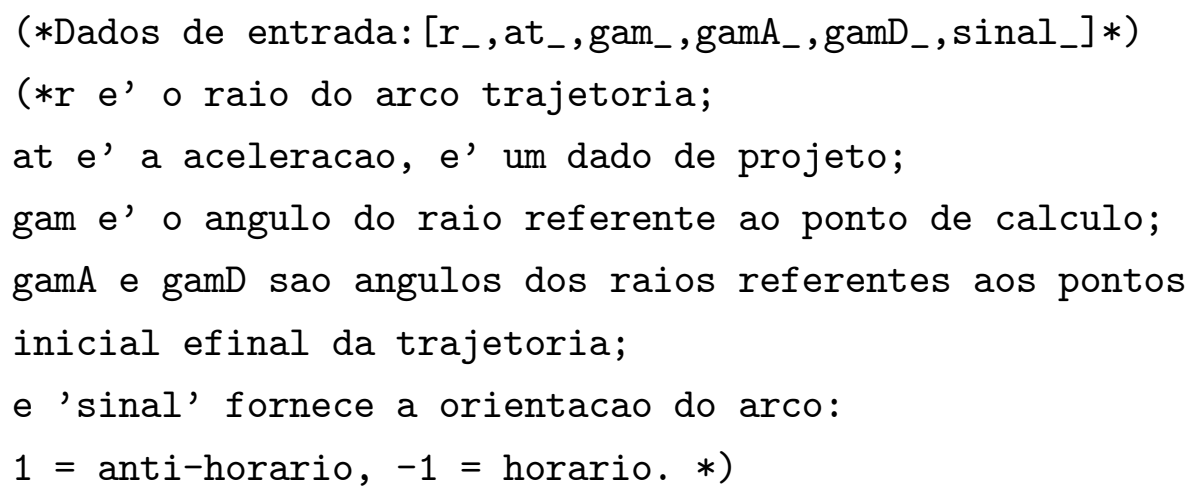




\section{Anexo F: PROGRAMA DAS VELOCIDADES E ACELERAÇÕES PARA TRAJETÓRIA CIRCULAR GERAL}

Neste anexo apresentamos o programa desenvolvido no software Mathematica que calcula as componentes da velocidade e da aceleração da garra para qualquer ponto, ou vários pontos igualmente espaçados, situados numa trajetória circular qualquer.

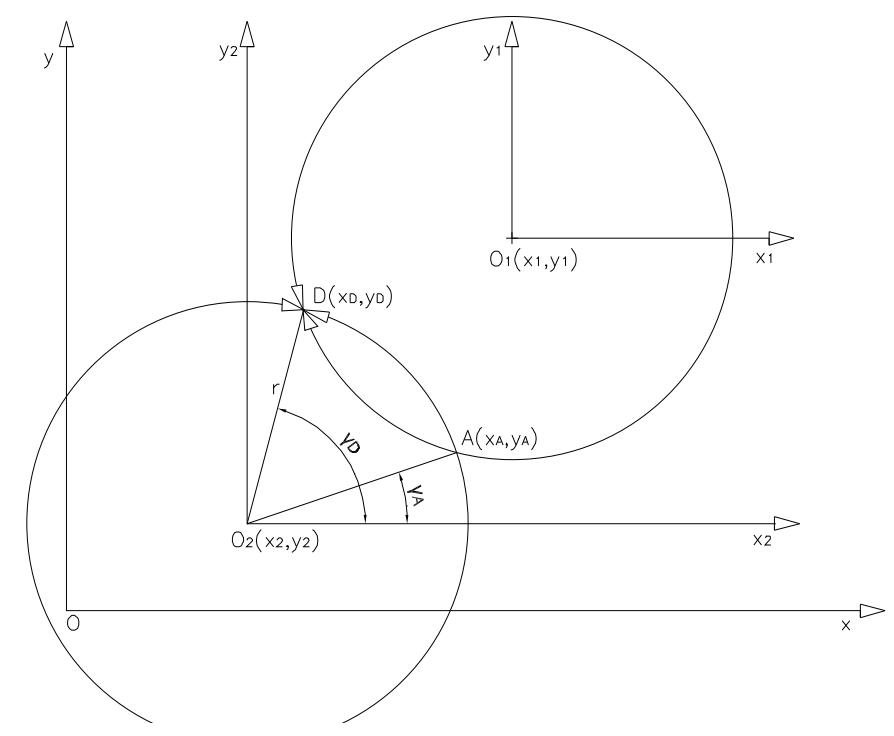

Figura 11.4: Anexo F: trajetória circular geral

Neste programa consideramos os seguintes dados de projeto:

-Velocidade máxima: $v_{\max }=1,0 \mathrm{~m} / \mathrm{s}$

-Módulo da aceleração igual da desaceleração: $10,0 \mathrm{~m} / \mathrm{s}^{2}$

Para a determinação das componentes da velocidade e aceleração em um ponto específico da trajetória devemos entrar com os seguintes dados:

-Raio do arco trajetória: $r$

-Aceleração tangencial: $a_{t}=10$

-Ângulo do raio referente ao ponto onde queremos achar os resultados: $\gamma$

-Coordenadas do ponto $A$, ponto inicial da trajetória: $\left(x_{A}, y_{A}\right)$

-Coordenadas do ponto $D$, ponto inicial da trajetória: $\left(x_{D}, y_{D}\right)$

-Orientação do arco: sinal $=1$ (sentido anti-horário), sinal $=-1$ (sentido horário) 
-Posição do centro do arco em relação à corda $A D:$ dir $=1$ (para centro do arco à direita de $\mathrm{AD})$, dir $=-1$ (centro do arco à esquerda)

O programa mostra os resultados na forma de um vetor: $\left\{\left\{v_{x}, v_{y}\right\},\left\{a_{x}, a_{y}\right\}\right\}$.

Para a determinação das componentes da velocidade e aceleração em $n$ pontos internos e igualmente espaçados da trajetória, devemos entrar com os seguintes dados:

-Raio do arco trajetória: $r$

-Aceleração tangencial: $a_{t}=10$

-Número de pontos internos e igualmente espaçados onde queremos achar os resultados: $n$

-Coordenadas do ponto $A$, ponto inicial da trajetória: $\left(x_{A}, y_{A}\right)$

-Coordenadas do ponto $D$, ponto inicial da trajetória: $\left(x_{D}, y_{D}\right)$

-Orientação do arco: sinal $=1$ (sentido anti-horário), sinal $=-1$ (sentido horário)

-Posição do centro do arco em relação à corda $A D:$ dir $=1$ (para centro do arco à direita de $\mathrm{AD})$, dir $=-1$ (centro do arco à esquerda de $\mathrm{AD}$ )

O programa mostra os resultados na forma de $n$ vetores: $\left\{\left\{v_{x}, v_{y}\right\},\left\{a_{x}, a_{y}\right\}\right\}$.

\section{LISTA DO PROGRAMA}

(*Determinacao das componentes da velocidade e aceleracao para qualquer ponto de uma trajetoria circular, COM CENTRO NAO COINCIDENTE COM A ORIGEM dos eixos de coordenadas.*)

(*Obs. : Todos os angulos devem ser no sentido antihorario a partir do eixo horizontal*)

(* 0 resultado e' um vetor com $\{\{\mathrm{vx}, \mathrm{vy}\},\{\mathrm{ax}, \mathrm{ay}\}\} . *$ )

(* Calculo de angulos com a horizontal, no sentido anti-horario.

0 e' o centro e A e' um ponto na semireta. *)

AngF $\left[O_{-}, A_{-}\right]:=\operatorname{Module}[\{$ ta, $P\}$,

$P=(A-0)$;

ta $=\operatorname{ArcCos}[\mathrm{P}[[1]] / \operatorname{Norm}[\mathrm{P}]]$;

If $[P[[2]]>=0, \operatorname{Return}[\mathrm{ta}], \operatorname{Return}[2 * \mathrm{Pi}-\mathrm{ta}]] ;]$

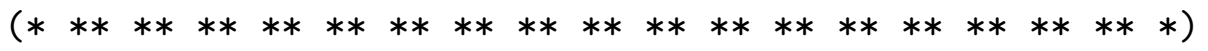

(* Essa funcao computa 0 angulo do arco entre alpha e beta no 
sentido desejado. E' usada no calculo dos comprimentos dos arcos.*) (* alpha e beta sao os angulos e sinal da' o sentido ...*)

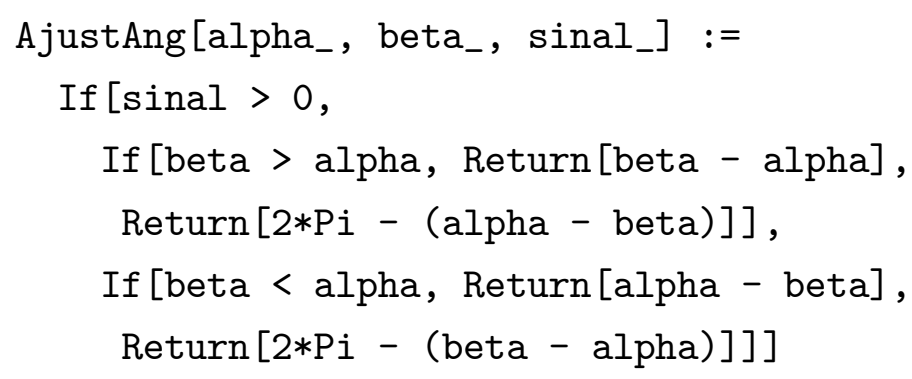




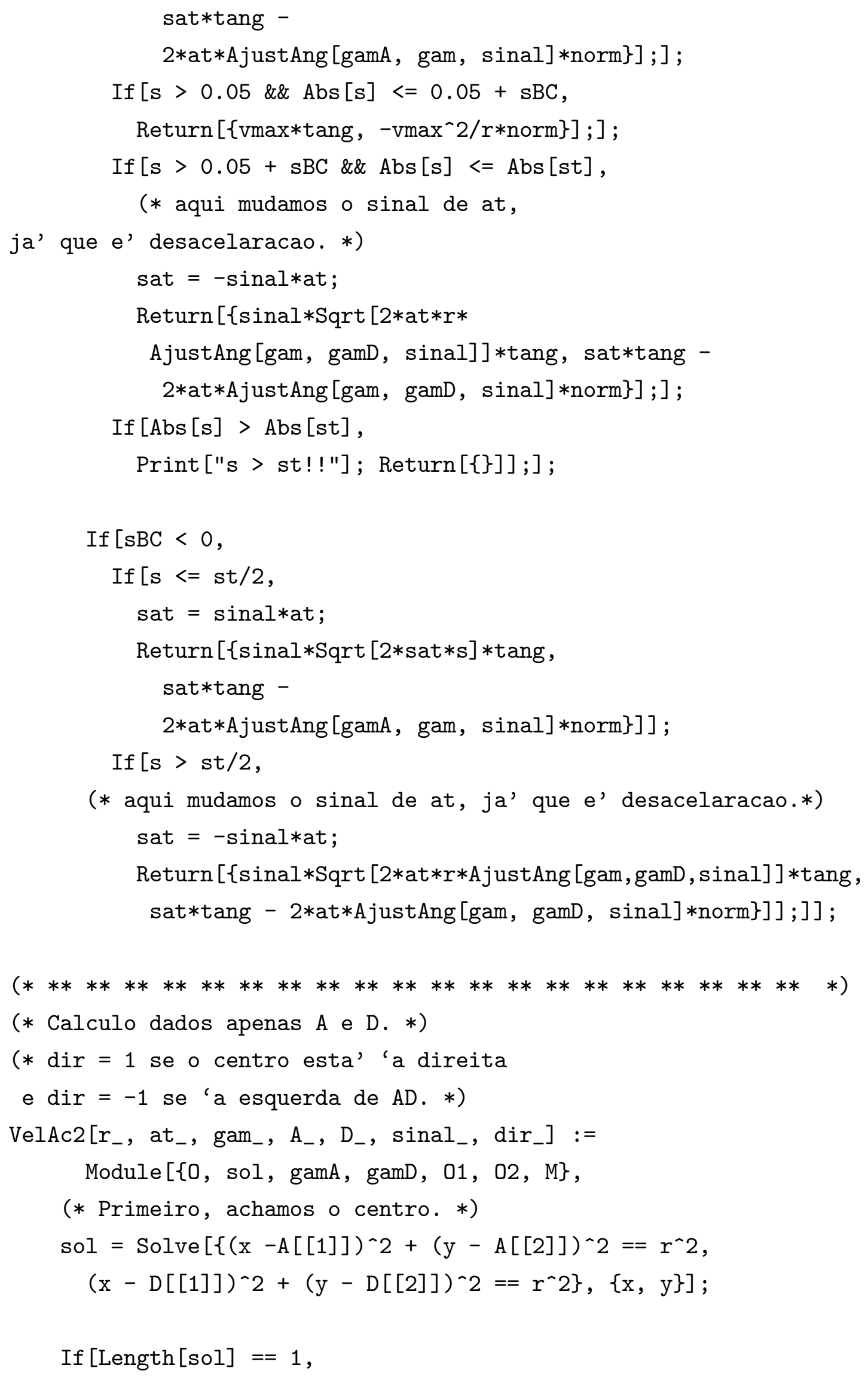




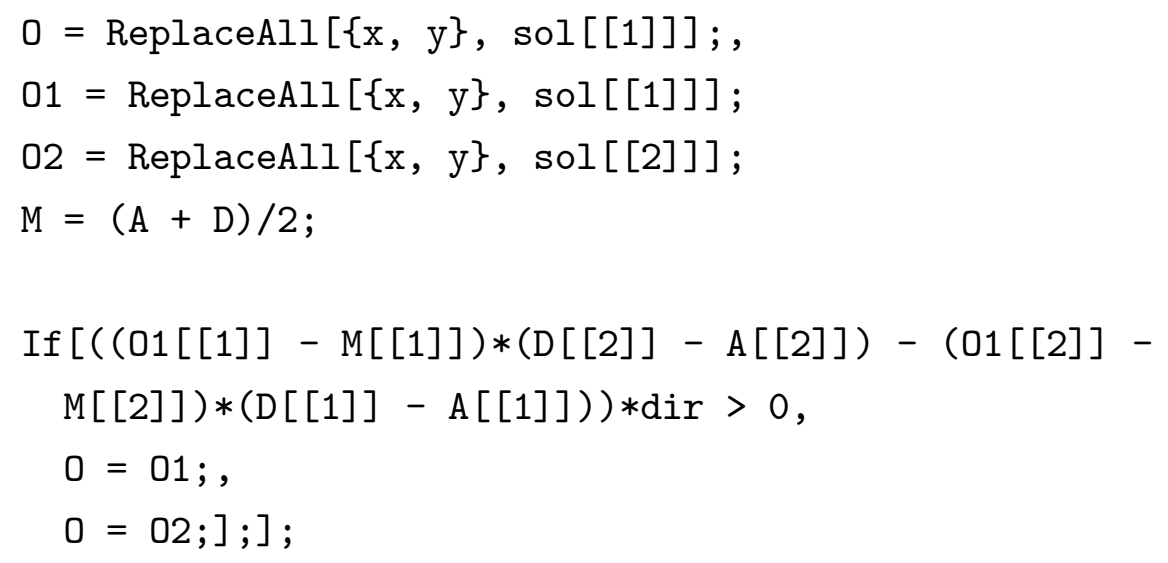

(* Agora, os angulos. *)

$\operatorname{gamA}=\operatorname{AngF}[0, \mathrm{~A}]$;

gamD = AngF $[0, D]$;

(* Chamamos a funcao anterior*)

$\operatorname{Return}[\operatorname{VelAc}[r$, at, gam, gamA, gamD, sinal]]]

$(* * * * * * * * * * * * * * * * * * * * * * * * * * * * * * * * * * *)$

(*CALCULO PARA UM PONTO P NA TRAJETORIA*)

(*Dados de entrada : $\left[r_{-}\right.$, at, gam $_{-},\{x A, y A\}$, $\left.\left.\{\mathrm{xD}, \mathrm{yD}\}, \operatorname{sinal}_{-}, \operatorname{dir}_{-}\right] *\right)$

(*onde, $r$ e' o raio do arco trajetoria;

at e' a aceleracao - e' um dado de projeto, sempre positivo;

gam e' o angulo do raio do ponto $P$, em relacao a horizontal;

$A(x A, y A)$ e' o ponto inicial e $D(x D, y D)$ e' o ponto final

do arco trajetoria;

'sinal' fornece a orientacao do arco : 1 = anti - horario,

-1 = horario; $*$ )

(*'dir' e' 1 se o centro do arco esta a direita da corda $\mathrm{AD}$ e e' -1 se estiver a esquerda*)

$\operatorname{VelAc2}[r$, at, gam, $\{x A, y A\},\{x D, y D\}$, sinal, $\operatorname{dir}]$

(*Desenvolvimento do programa para $\mathrm{n}$ pontos internos

a trajetoria e igualmente espacados*)

(*Usamos uma proporcao do ang. total ao inves de gama.*)

$\operatorname{VelAc3}\left[r_{-}\right.$, at_, prop_, $A_{-}, D_{-}$, sinal_, dir_] := 


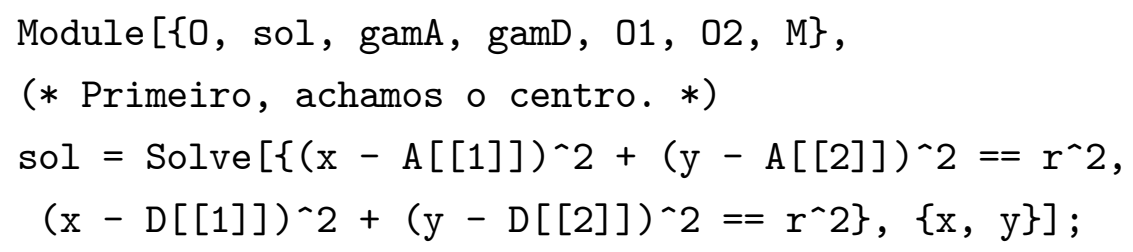




\section{Anexo G: PROGRAMA EQUAÇÕES DAS VELOCIDADES E ACELERAÇÕES}

Neste anexo apresentamos o programa executado no software Mathematica que deduz as equações para o cálculo das velocidades angulares $\dot{\theta}_{1}=u_{1}, \dot{\theta}_{2}=u_{2}$, $\dot{\theta}_{3}=u_{3}, \dot{\theta}_{4}=u_{4}, \dot{\theta}_{5}=u_{5}$ e $\dot{q}_{6}=u_{6}$ e das acelerações angulares $\ddot{\theta}_{1}=\dot{u}_{1}, \ddot{\theta}_{2}=\dot{u}_{2}$, $\ddot{\theta}_{3}=\dot{u}_{3}, \ddot{\theta}_{4}=\dot{u}_{4}, \ddot{\theta}_{5}=\dot{u}_{5}$ e $\ddot{q}_{6}=\dot{u}_{6}$ utilizando as seguintes expressões:

Determinação de $u_{5}$ e $u_{6}$

$$
\begin{aligned}
& V_{J x}=c_{5} \dot{q}_{6}-q_{6} s_{5} \dot{\theta}_{5}=c_{5} u_{6}-q_{6} s_{5} u_{5} \\
& V_{J y}=s_{5} \dot{q}_{6}+q_{6} c_{5} \dot{\theta}_{5}=s_{5} u_{6}+q_{6} c_{5} u_{5}
\end{aligned}
$$

Determinação de $\dot{u}_{5}$ e $\dot{u_{6}}$.

$$
\begin{gathered}
a_{J x}=-q_{6} s_{5} \dot{u}_{5}+c_{5} \dot{u_{6}}-2 u_{5} u_{6} s_{5}-q_{6}\left(u_{5}\right)^{2} c_{5} \\
a_{J y}=q_{6} c_{5} \dot{u_{5}}+s_{5} \dot{u_{6}}+2 u_{5} u_{6} c_{5}-q_{6}\left(u_{5}\right)^{2} s_{5}
\end{gathered}
$$

Determinação de $u_{1} \mathrm{e} u_{2}$

$$
\begin{gathered}
V_{J x}=-L_{A} s_{1} u_{1}-L_{B} s_{12}\left(u_{1}+u_{2}\right)-L_{J} c_{5} u_{5} \\
V_{J y}=L_{A} c_{1} u_{1}+L_{B} c_{12}\left(u_{1}+u_{2}\right)-L_{J} s_{5} u_{5}
\end{gathered}
$$

Determinação de $\dot{u_{1}}$ e $\dot{u_{2}}$

$$
\begin{gathered}
a_{J x}=-L_{A} c_{1}\left(u_{1}\right)^{2}-L_{A} s_{1} \dot{u_{1}}-L_{B} c_{12}\left(u_{1}+u_{2}\right)^{2}- \\
L_{B} s_{12}\left(\dot{u_{1}}+\dot{u_{2}}\right)+L_{J} s_{5}\left(u_{5}\right)^{2}-L_{J} c_{5} \dot{u_{5}} \\
a_{J y}=-L_{A} s_{1}\left(u_{1}\right)^{2}+L_{A} c_{1} \dot{u_{1}}-L_{B} s_{12}\left(u_{1}+u_{2}\right)^{2}+ \\
L_{B} c_{12}\left(\dot{u_{1}}+\dot{u}_{2}\right)-L_{J} c_{5}\left(u_{5}\right)^{2}-L_{J} s_{5} \dot{u_{5}}
\end{gathered}
$$

Determinação de $u_{3} \mathrm{e} u_{4}$.

$$
\begin{gathered}
V_{J x}=-L_{D} s_{3} u_{3}-L_{E} s_{34}\left(u_{3}+u_{4}\right)+L_{J} c_{5} u_{5} \\
V_{J y}=L_{D} c_{3} u_{3}+L_{E} c_{34}\left(u_{3}+u_{4}\right)+L_{J} s_{5} u_{5}
\end{gathered}
$$

Determinação de $\dot{u}_{3}$ e $\dot{u_{4}}$. 


$$
\begin{gathered}
a_{J x}=-L_{D} c_{3}\left(u_{3}\right)^{2}-L_{D} s_{3} \dot{u_{3}}-L_{E} c_{34}\left(u_{3}+u_{4}\right)^{2}- \\
L_{E} s_{34}\left(\dot{u} 3+\dot{u}_{4}\right)-L_{J} s_{5}\left(u_{5}\right)^{2}+L_{J} c_{5} \dot{u_{5}} \\
a_{J y}=-L_{D} s_{3}\left(u_{3}\right)^{2}+L_{D} c_{3} \dot{u_{3}}-L_{E} s_{34}\left(u_{3}+u_{4}\right)^{2}+ \\
L_{E} c_{34}\left(\dot{u_{3}}+\dot{u_{4}}\right)-L_{J} c_{5}\left(u_{5}\right)^{2}-L_{J} s_{5} \dot{u_{5}}
\end{gathered}
$$

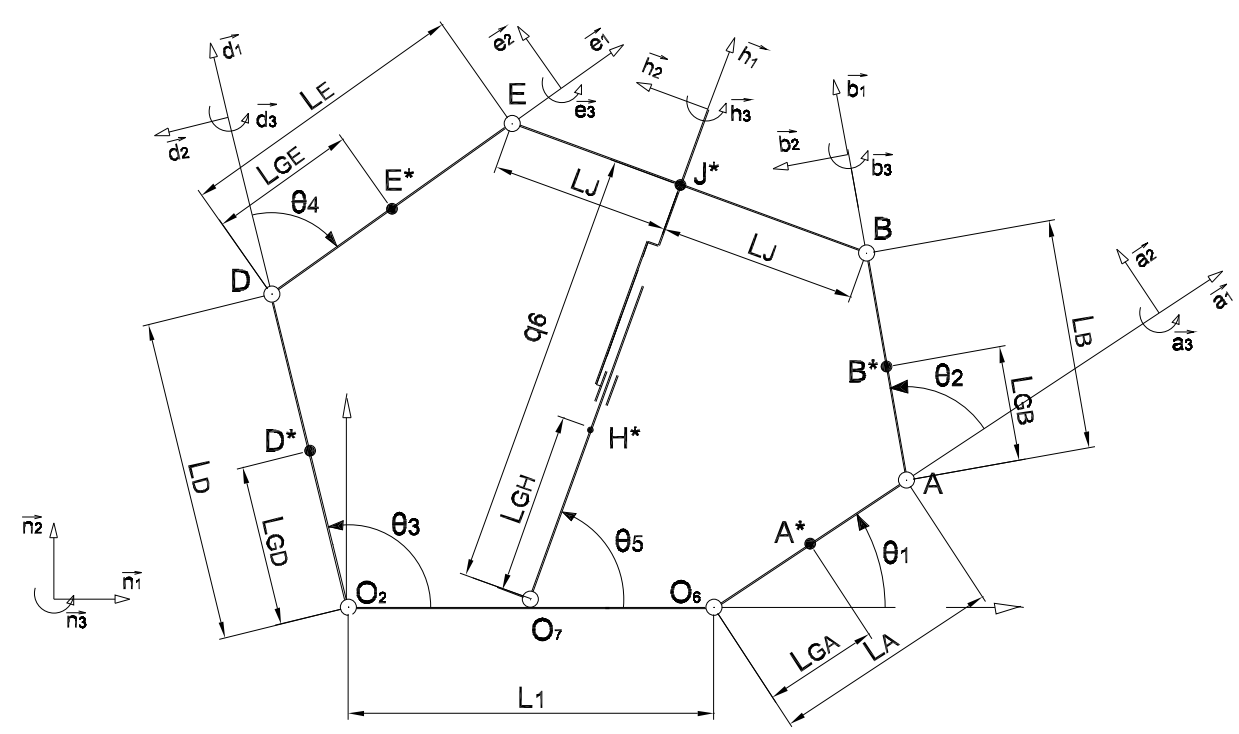

Figura 11.5: Anexo G: diagrama cinemático

\section{LISTA DO PROGRAMA}

(*Kane5. Determinacao das equacoes das velocidades e aceleracoes $*$ )

(* Determinacao de $\mathrm{t71}$ e q81*)

Solve $[\{\mathrm{VJx}==\mathrm{q} 81 * \mathrm{c} 7-\mathrm{q} 8 * \mathrm{~s} 7 * \mathrm{t} 71$,

$\mathrm{VJy}==\mathrm{q} 81 * \mathrm{~s} 7+\mathrm{q} 8 * \mathrm{c} 7 * \mathrm{t} 71\},\{\mathrm{t} 71, \mathrm{q} 81\}]$

(* Determinacao de t72 e q82*)

Solve $[\{\mathrm{aJx}==\mathrm{q} 82 * \mathrm{c} 7-2 *$

$\mathrm{q} 81 * \mathrm{~s} 7 * \mathrm{t} 71-\mathrm{q} 8 * \mathrm{c} 7 * \mathrm{t} 71^{\wedge} 2-\mathrm{q} 8 * \mathrm{~s} 7 * \mathrm{t} 72$, aJy $==\mathrm{q} 82 *$

$\mathrm{s} 7+2 * \mathrm{q} 81 * \mathrm{c} 7 * \mathrm{t} 71-\mathrm{q} 8 * \mathrm{~s} 7 * \mathrm{t} 71 \wedge 2+\mathrm{q} 8 * \mathrm{c} 7 * \mathrm{t} 72\},\{\mathrm{t} 72, \mathrm{q} 82\}]$

(*Determinacao de t11 e t21 *)

Solve $[\{\mathrm{VJx}==-\mathrm{LA} * \mathrm{~s} 1 * \mathrm{t} 11-\mathrm{LB} * \mathrm{~s} 12 *(\mathrm{t} 11+\mathrm{t} 21)-\mathrm{LJ} * \mathrm{c} 7 * \mathrm{t} 71$, 


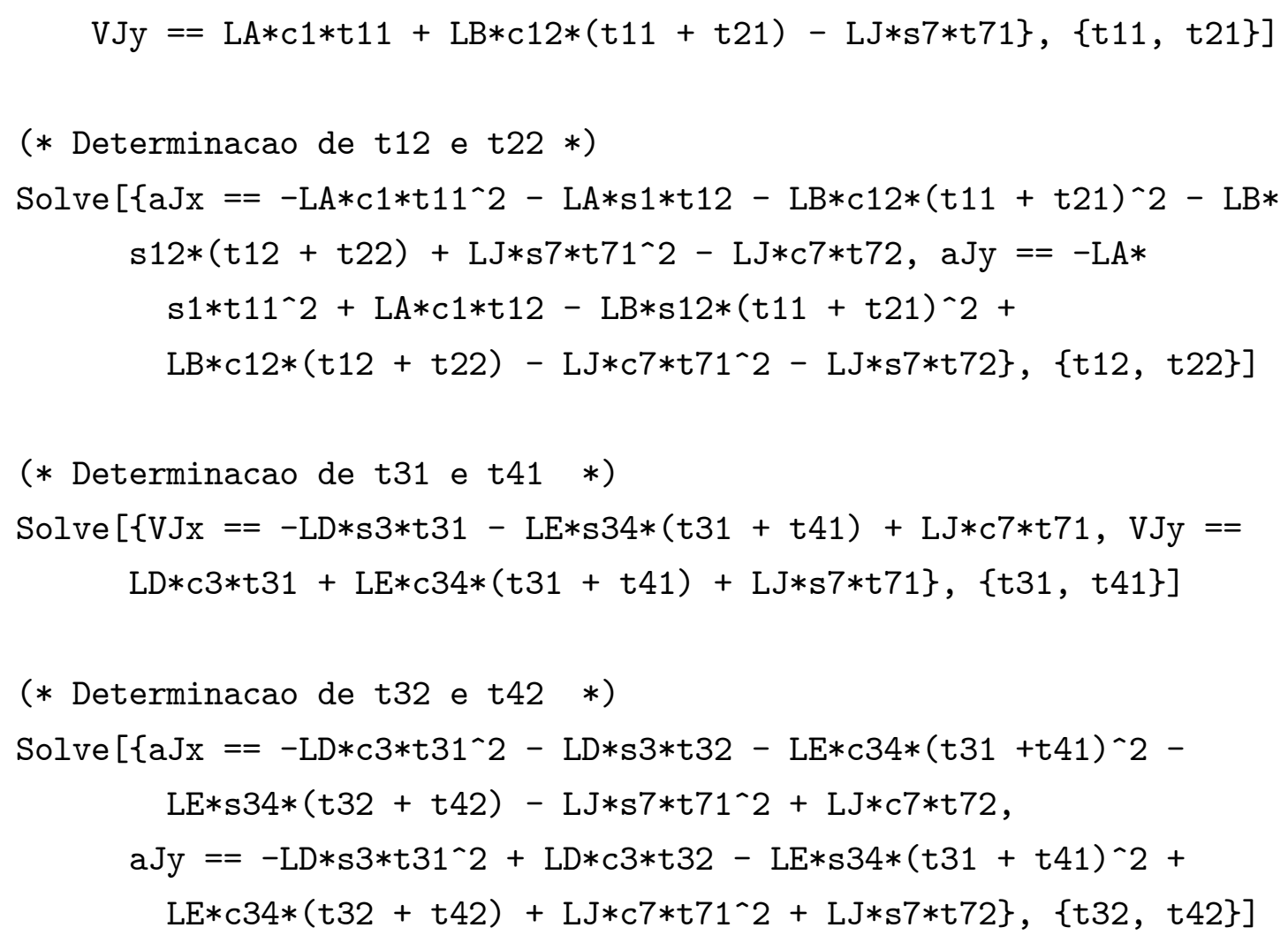




\section{Anexo H: PROGRAMA CÁlCULO VELOCIDADES E ACELERAÇÕES}

Neste anexo apresentamos o programa elaborado no software Mathematica que, com as equações deduzidas no anexo $\mathrm{G}$, determina os valores numéricos das velocidades $\dot{\theta}_{1}=u_{1}, \dot{\theta}_{2}=u_{2}, \dot{\theta}_{3}=u_{3}, \dot{\theta}_{4}=u_{4}, \dot{\theta}_{5}=u_{5}$ e $\dot{q}_{6}=u_{6}$ e das acelerações $\ddot{\theta}_{1}=\dot{u}_{1}, \ddot{\theta}_{2}=\dot{u_{2}}, \ddot{\theta}_{3}=\dot{u}_{3}, \ddot{\theta}_{4}=\dot{u}_{4}, \ddot{\theta}_{5}=\dot{u}_{5}$ e $\ddot{q}_{6}=\dot{u}_{6}$; e com as equações 6.13 a 6.24 determina as acelerações dos CG dos elos, isto é, $A_{A}^{n}, A_{A}^{t}, A_{B}^{n}, A_{B}^{t}, A_{D}^{n}, A_{D}^{t}$, $A_{E}^{n}, A_{E}^{t}, A_{H}^{n}, A_{H}^{t}, A_{J}^{n}$ e $A_{J}^{t}$.

Os dados de entrada são:

-Ângulos dos elos: $\theta_{1}, \theta_{2}, \theta_{3}, \theta_{4}$ e $\theta_{5}$. (Sentido anti-horário é positivo)

-Comprimento dos elos: $L_{A}, L_{B}, L_{D}, L_{E}, L_{J}$, e $q_{6}$.

-Distâncias dos CG: $L_{G A}, L_{G B}, L_{G D}, L_{G E}$ e $L_{G H}$.

-Componentes da velocidade da garra: $V_{J x}$ e $V_{J y}$

-Componentes da aceleração da garra: $a_{J x}$ e $a_{J y}$.

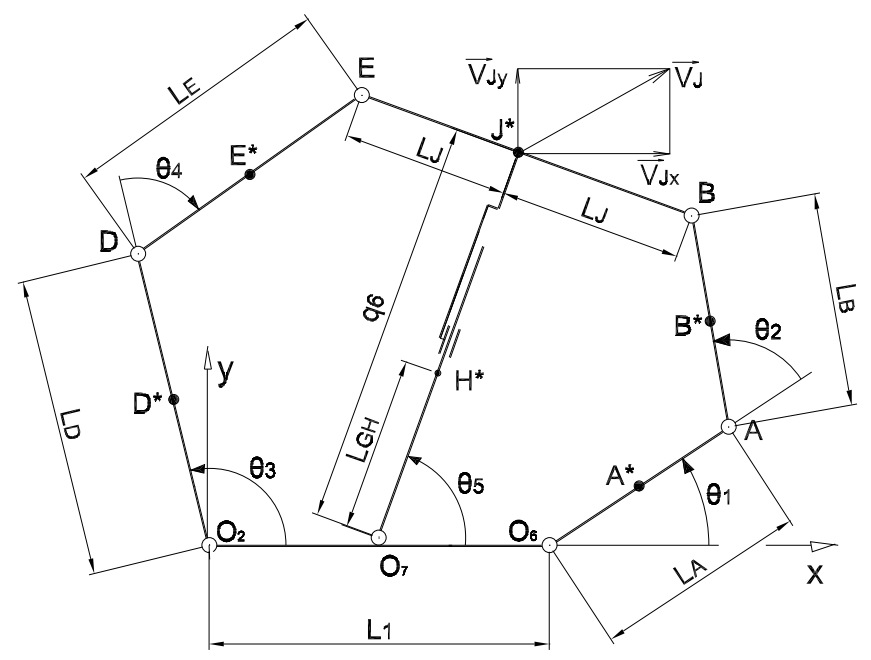

Figura 11.6: Anexo H: diagrama cinemático

\section{LISTA DO PROGRAMA}

(*ANEXO H : Det. das Velocidades e Acel. dos elos *)

(* Resultado : $\{\mathrm{t} 11, \mathrm{t} 12, \mathrm{t} 21, \mathrm{t} 22, \mathrm{t} 31, \mathrm{t} 32, \mathrm{t} 41, \mathrm{t} 42$, t51, t52, q61, q62, AAn, AAt, ABn, ABt, $\mathrm{ADn}, \mathrm{ADt}$, AEn, AEt, AHn, AHt, AJn, AJt\} *) 


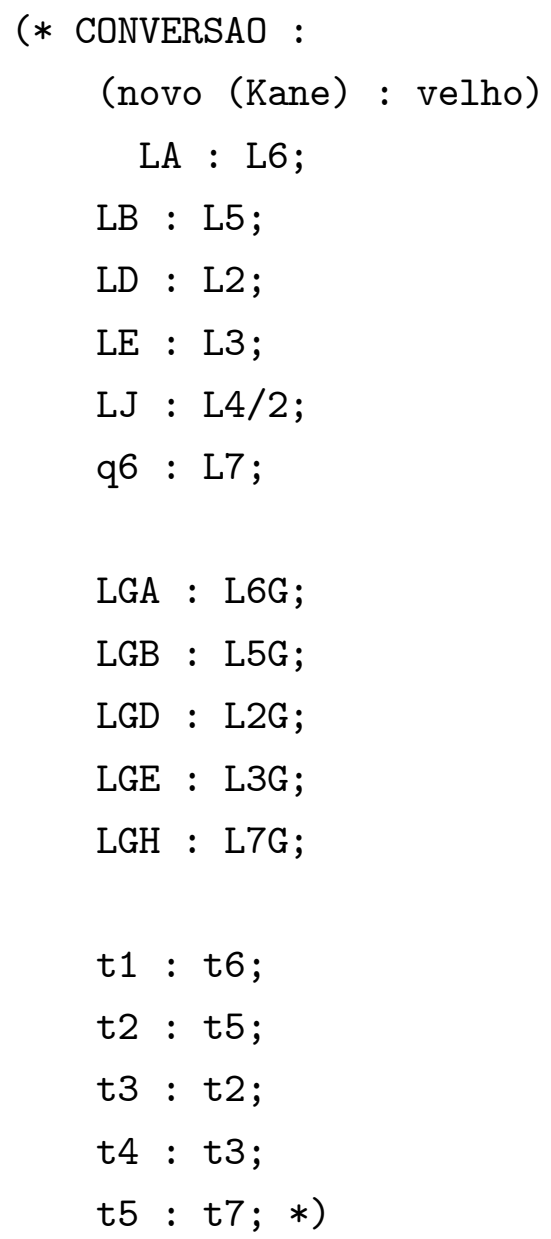

FctH $\left[\mathrm{t} 1_{-}, \mathrm{t2} 2_{-}, \mathrm{t} 3_{-}, \mathrm{t} 4_{-}, \mathrm{t5} 5_{-}\right.$,

$\mathrm{LA}_{-}, \mathrm{LB}_{-}, \mathrm{LD} \mathrm{L}_{-}, \mathrm{LE} \mathrm{E}_{-}, \mathrm{LJ} \mathrm{J}_{-}, \mathrm{q} 6_{-}$,

$\mathrm{LGA}_{-}, \mathrm{LGB}_{-}, \mathrm{LGD}_{-}, \mathrm{LGE}_{-}, \mathrm{LGH}_{-}$,

$\left.\mathrm{VJx}_{-}, \mathrm{VJy}_{-}, \mathrm{aJx}_{-}, \mathrm{aJy}_{-}\right]:=$

Module $[\{c 1, c 2, c 3, c 4, c 5, \mathrm{~s} 1, \mathrm{~s} 2, \mathrm{~s} 3, \mathrm{~s} 4, \mathrm{~s} 5$, s12, s34, c12, c34, calf, salf, clam, slam, sol, t51, t52, q61, q62, t11, t12, t21, t22, t31, t32, t41, t42, $A A n, A A t, A B n, A B t, A D n, A D t, A E n, A E t, A H n, A H t, A J n, A J t\}$,

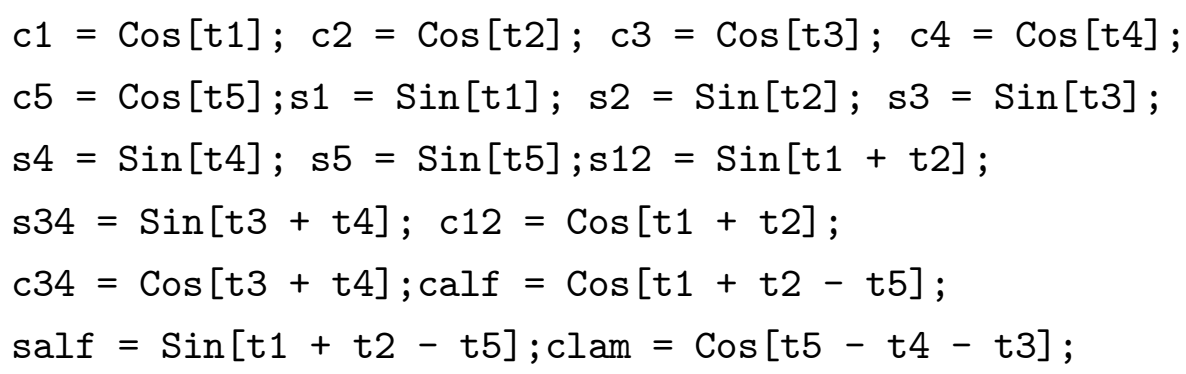




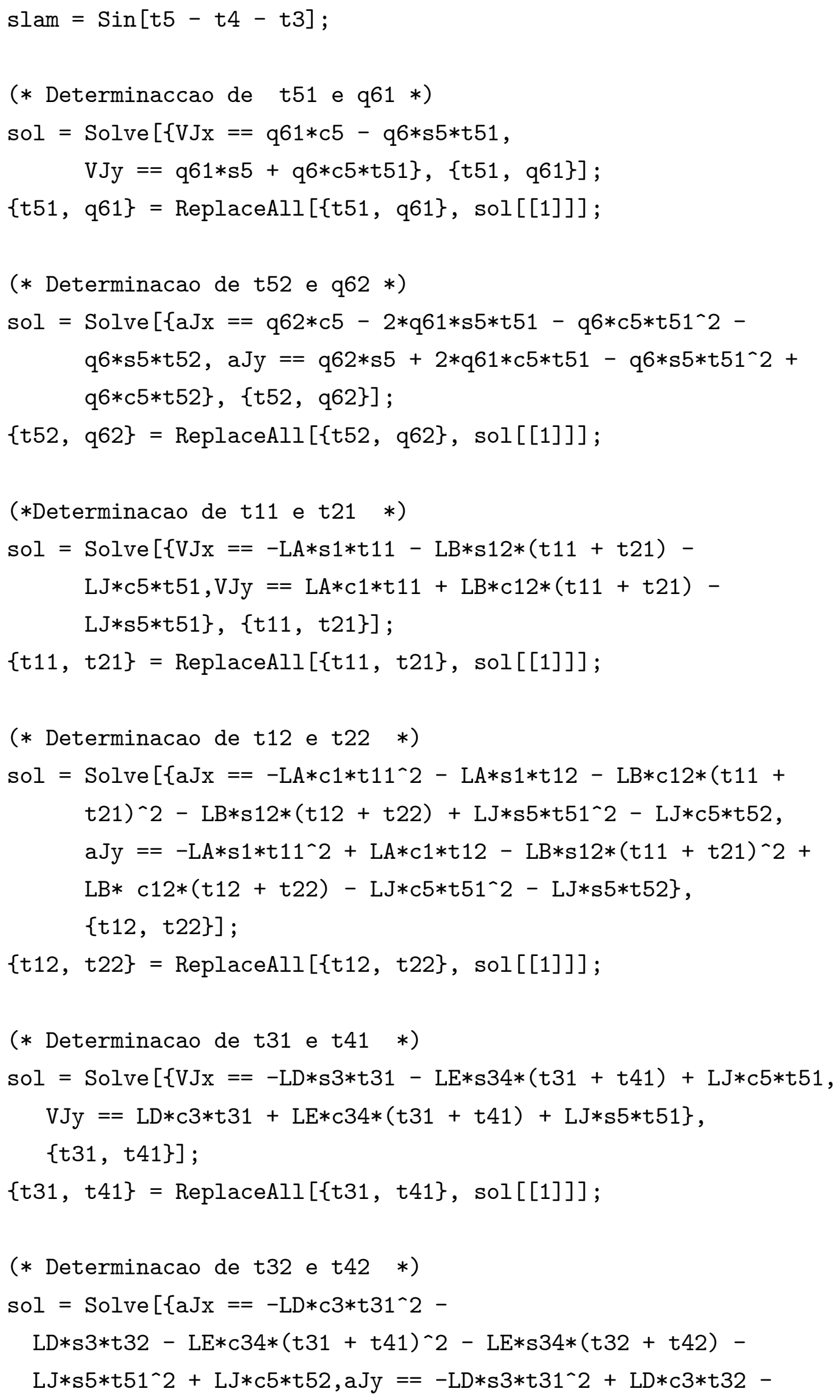




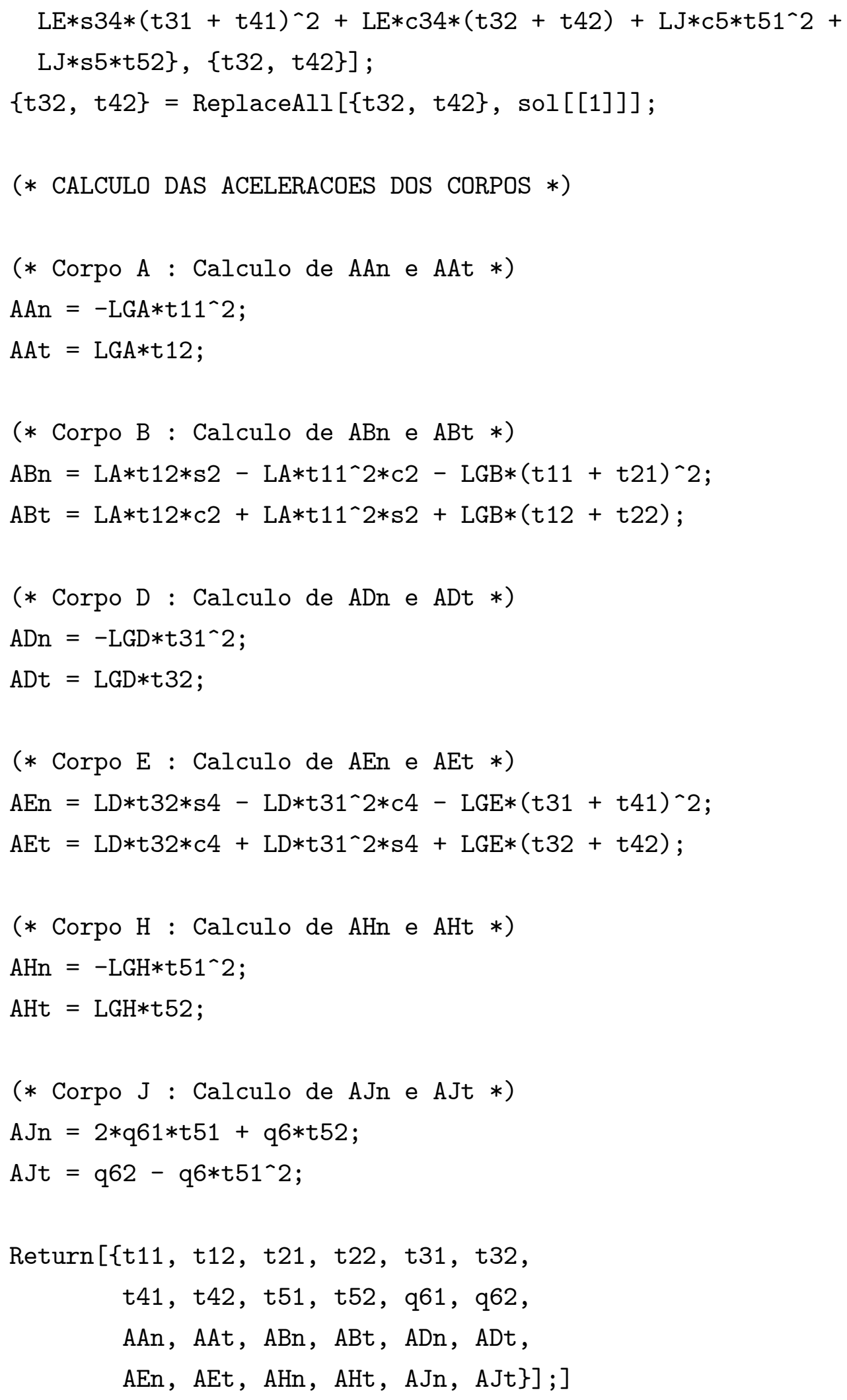




\section{Anexo I: PROGRAMA ANÁLISE ESTÁTICA}

Neste anexo apresentamos o programa feito no software Mathematica para o qual devem ser fornecidos os seguintes dados:

-Comprimento dos elos: $L_{1}$ a $L_{6}$.

-Comprimento da cadeia passiva e seu ângulo de inclinação: $L_{7}$ e $\theta_{7}$.

-Pesos dos elos: $P_{1}$ a $P_{7}$.

-Posições dos centros de gravidade: $L_{2 G}, L_{3 G}, L_{5 G}, L_{6 G}$, e $L_{7 G}$.

-Os pesos dos contrapesos: $W_{2}, W_{3}, W_{5}, W_{6}$ e $W_{7}$.

-As posições dos contrapesos: $L_{2 P}, L_{3 P}, L_{5 P}, L_{6 P}$ e $L_{7 P}$.

-Força externa $F$ e sua inclinação $\beta$

-O torque externo $M$.

O programa determina:

-Os ângulos de entrada: $\theta_{2}$ e $\theta_{6}$.

-Os ângulos dos elos $L_{3}$ e $L_{5}: \theta_{3}$ e $\theta_{5}$.

-As componentes das forças de reação: $F_{1 x}, F_{1 y}, F_{7 x}, F_{7 y}, F_{8 x}$ e $F_{8 y}$.

-Os momentos de reação: $M_{2}$ e $M_{8}$.

-Verifica a existência de singularidades e informa seus tipos.

-Traça o gráfico do mecanismo

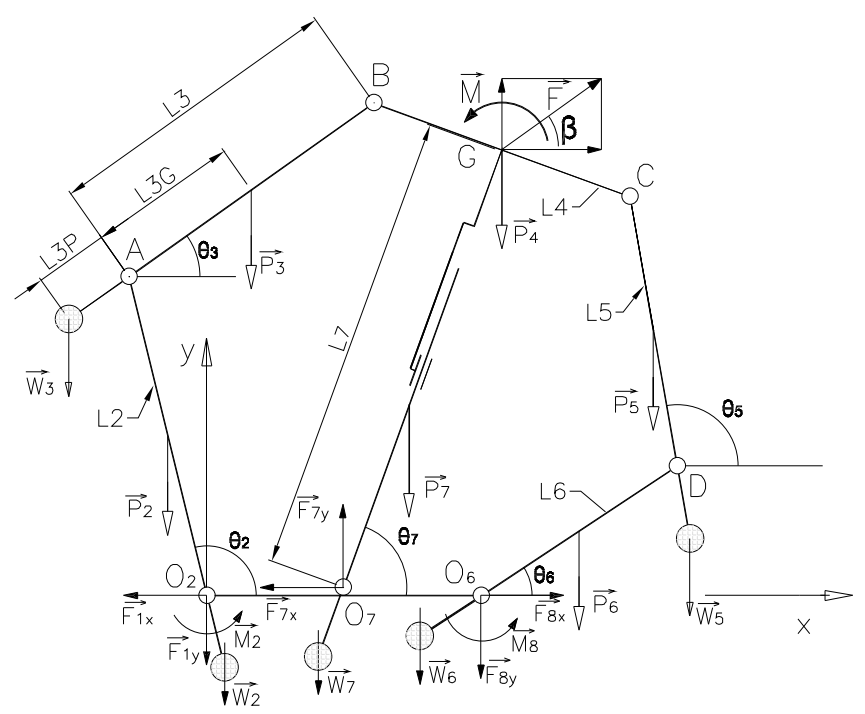

Figura 11.7: Anexo I: diagrama cinemático 


\section{LISTA DO PROGRAMA}

(*ANEXO I : ANALISE ESTATICA *)

(* Resultado : $\{\mathrm{F} 1 \mathrm{x}, \mathrm{F} 1 \mathrm{y}, \mathrm{F} 2 \mathrm{x}, \mathrm{F} 2 \mathrm{y}, \mathrm{F} 3 \mathrm{x}$,

F3y, F4, F5x, F5y, F6x, F6y, F7x, F7y, F8x, F8y, M2, M4, M8\} *)
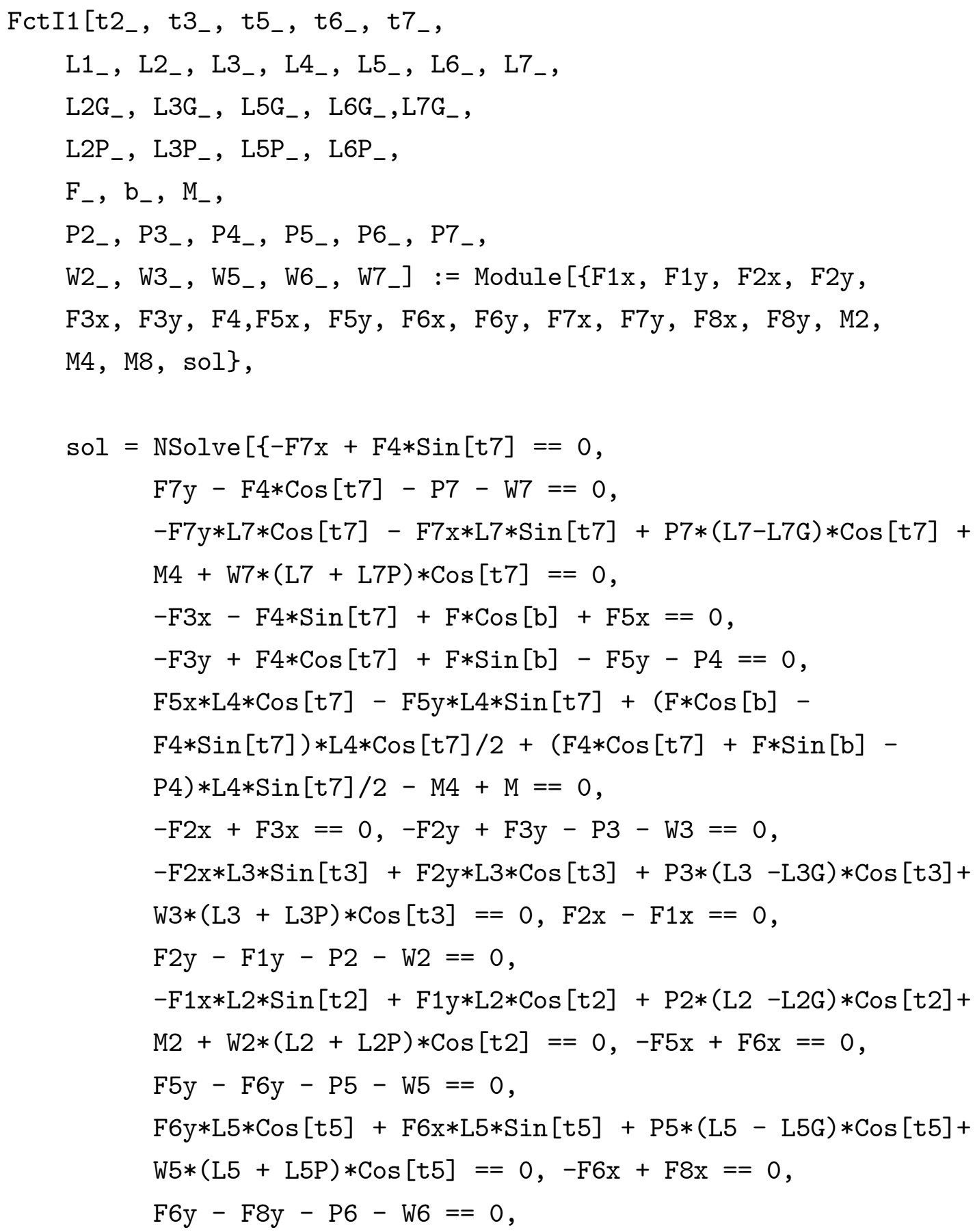


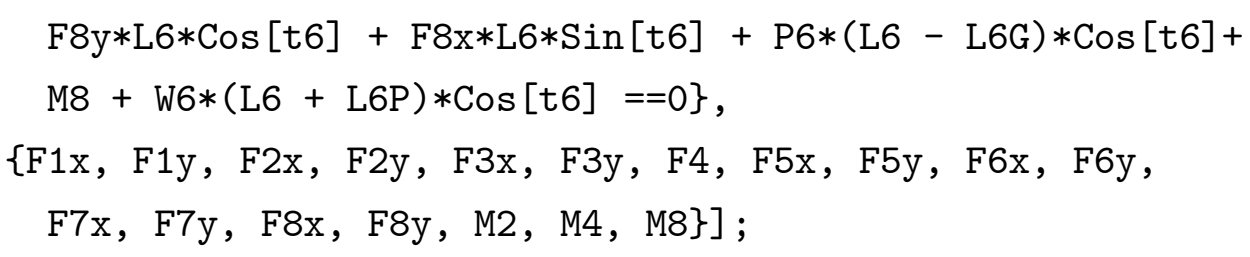

Return [ReplaceAll $[\{F 1 x, F 1 y, F 2 x, F 2 y, F 3 x, F 3 y, F 4, F 5 x, F 5 y$, F6x, F6y, F7x, F7y, F8x, F8y, M2, M4, M8\}, sol[[1]]]];]

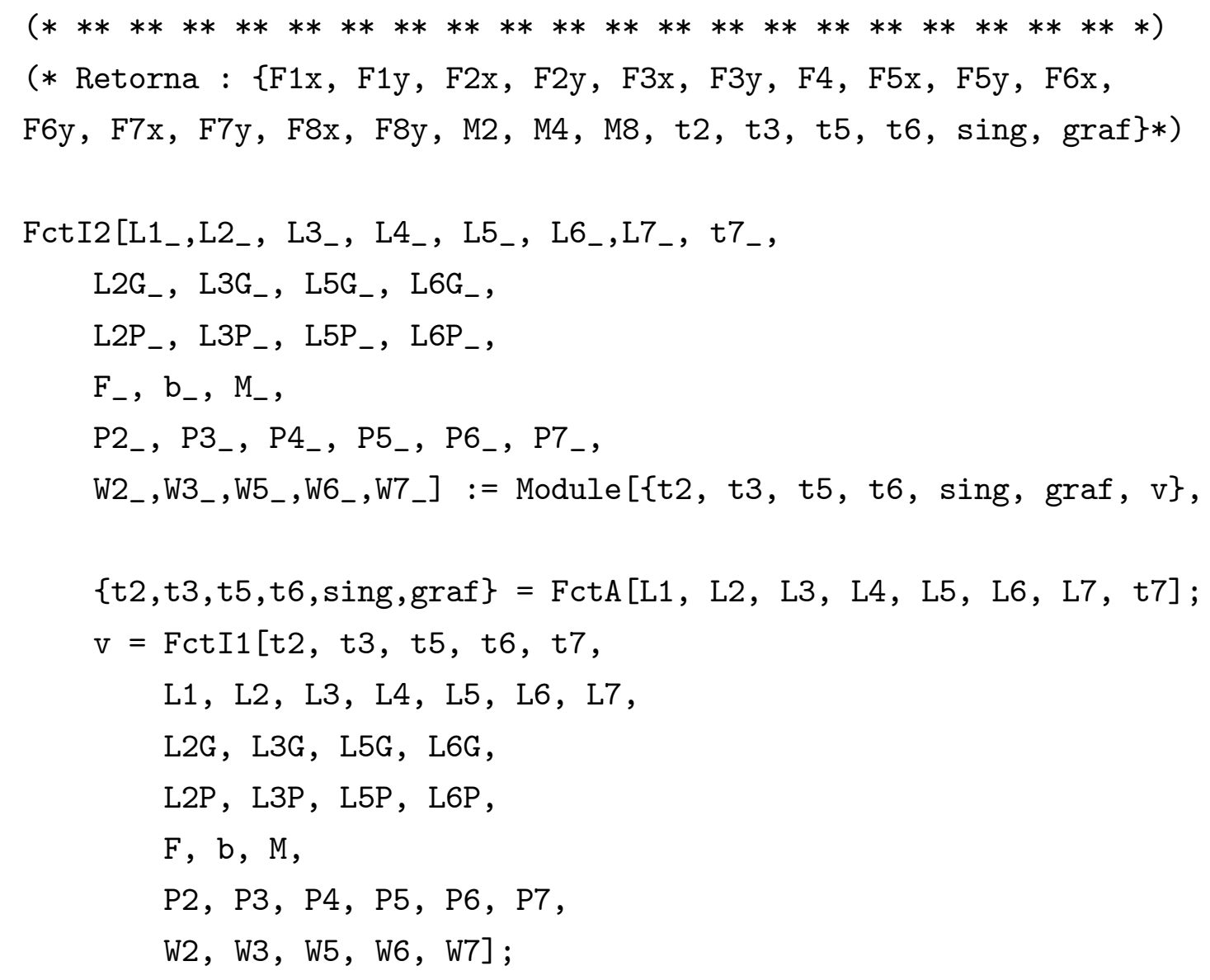

$\operatorname{Return}[\mathrm{Join}[\mathrm{v},\{\mathrm{t} 2, \mathrm{t} 3, \mathrm{t5}, \mathrm{t} 6, \mathrm{sing}, \operatorname{graf}\}]] ;]$ 


\section{Anexo J: PROGRAMA EQUAÇÕES DINÂMICA 1}

Neste anexo apresentamos o programa Mathematica que deduz as equações dos torques de reação dos atuadores, $T_{1}$ e $T_{1}^{D}$, e as forças $F_{5}, F_{5}^{D}, F_{6}$ e $F_{6}^{D}$ que atuam nas juntas da plataforma móvel.

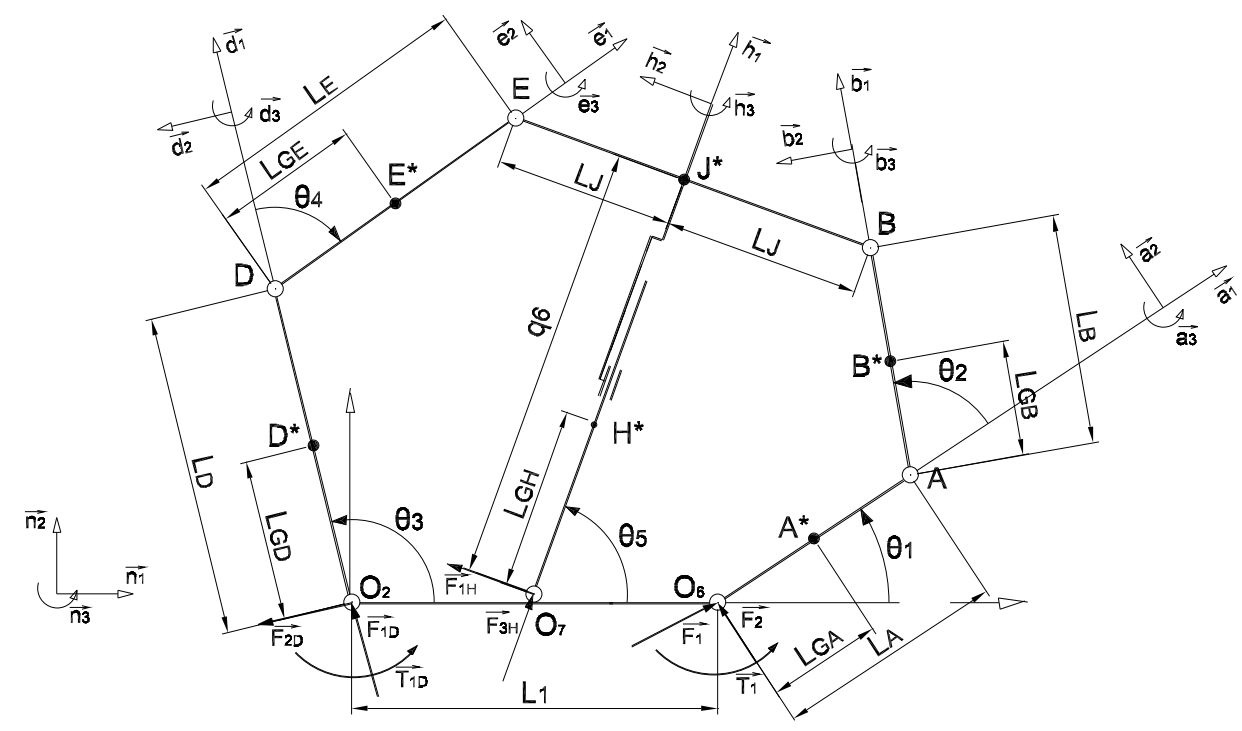

Figura 11.8: Anexo J: diagrama cinemático

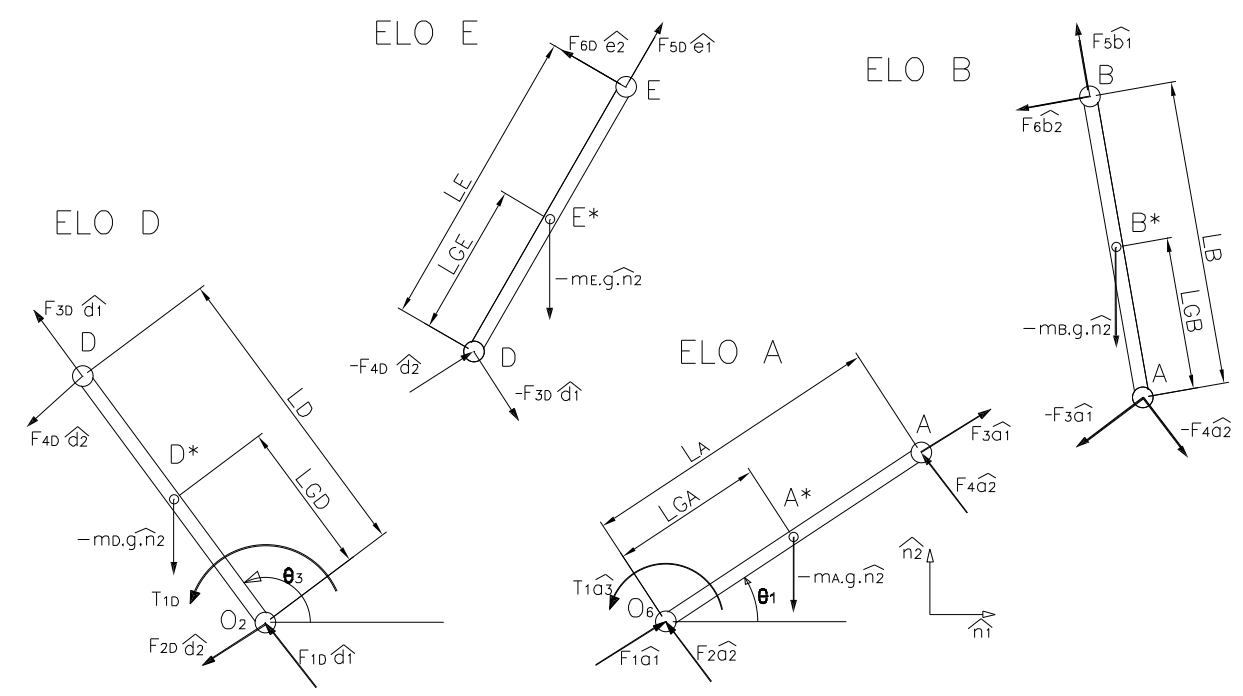

Figura 11.9: Anexo J: diagramas de corpo livre 
Utilizamos as equações deduzidas pelo método de Kane:

1. Equação (AB1)

$$
\begin{aligned}
& T_{1}+F_{5} L_{A} s_{2}+F_{6} L_{A} c_{2}+F_{6} L_{B}-m_{A} g L_{G A} c_{1}-m_{B} g L_{A} c_{1}-m_{B} g L_{G B} c_{12} \\
= & \left(I_{A}+I_{B}\right) \dot{u_{1}}+I_{B} \dot{u_{2}}+m_{A} L_{G A} A_{A}^{t}+m_{B}\left(A_{B}^{n} L_{A} s_{2}+A_{B}^{t} L_{A} c_{2}+A_{B}^{t} L_{G B}\right)
\end{aligned}
$$

2. Equação (AB2)

$$
F_{6} L_{B}-m_{B} g L_{G B} c_{12}=m_{B} L_{G B} A_{B}^{t}+I_{B}\left(\dot{u_{1}}+\dot{u_{2}}\right)
$$

3. Equação (DE3)

$$
\begin{aligned}
& T_{1}^{D}+F_{5}^{D} L_{D} s_{4}+F_{6}^{D} L_{D} c_{4}+F_{6}^{D} L_{E}-m_{D} g L_{G D} c_{3}-m_{E} g L_{D} c_{3}-m_{E} g L_{G E} c_{34} \\
& =\left(I_{D}+I_{E}\right) \dot{u_{3}}+I_{E} \dot{u}_{4}+m_{D} L_{G D} A_{D}^{t}+m_{E}\left(A_{E}^{n} L_{D} s_{4}+A_{E}^{t} L_{D} c_{4}+A_{E}^{t} L_{G E}\right)
\end{aligned}
$$

4. Equação (DE4)

$$
F_{6}^{D} L_{E}-m_{E} g L_{G E} c_{34}=m_{E} L_{G E} A_{E}^{t}+I_{E}\left(\dot{u_{3}}+\dot{u_{4}}\right)
$$

5. Equação (HJ5)

$$
\begin{gathered}
-F_{5}\left(L_{j} c_{\alpha}+q_{6} s_{\alpha}\right)+F_{5}^{D}\left(L_{j} c_{\lambda}+q_{6} s_{\lambda}\right)+F_{6}\left(L_{j} s_{\alpha}-q_{6} c_{\alpha}\right)+ \\
F_{6}^{D}\left(L_{j} s_{\lambda}-q_{6} c_{\lambda}\right)-m_{H} g L_{G H} c_{5}-m_{J} g q_{6} c_{5} \\
=m_{H} L_{G H} A_{H}^{t}+m_{J} q_{6} A_{J}^{n}+\left(I_{H}+I_{J}\right) \dot{u}_{5}
\end{gathered}
$$

6. Equação (HJ6)

$$
\begin{gathered}
-F_{6}^{D} s_{\lambda}-F_{5}^{D} c_{\lambda}-F_{5} c_{\alpha}+F_{6} s_{\alpha}-m_{J} g s_{5} \\
=m_{J} A_{J}^{t}
\end{gathered}
$$




\section{LISTA DO PROGRAMA}

((*KANE1, Det. das equas dos torques T1 e T1D e forcas F5, F5D, $\mathrm{F} 6$ e $\mathrm{F} 6 \mathrm{D} *)$

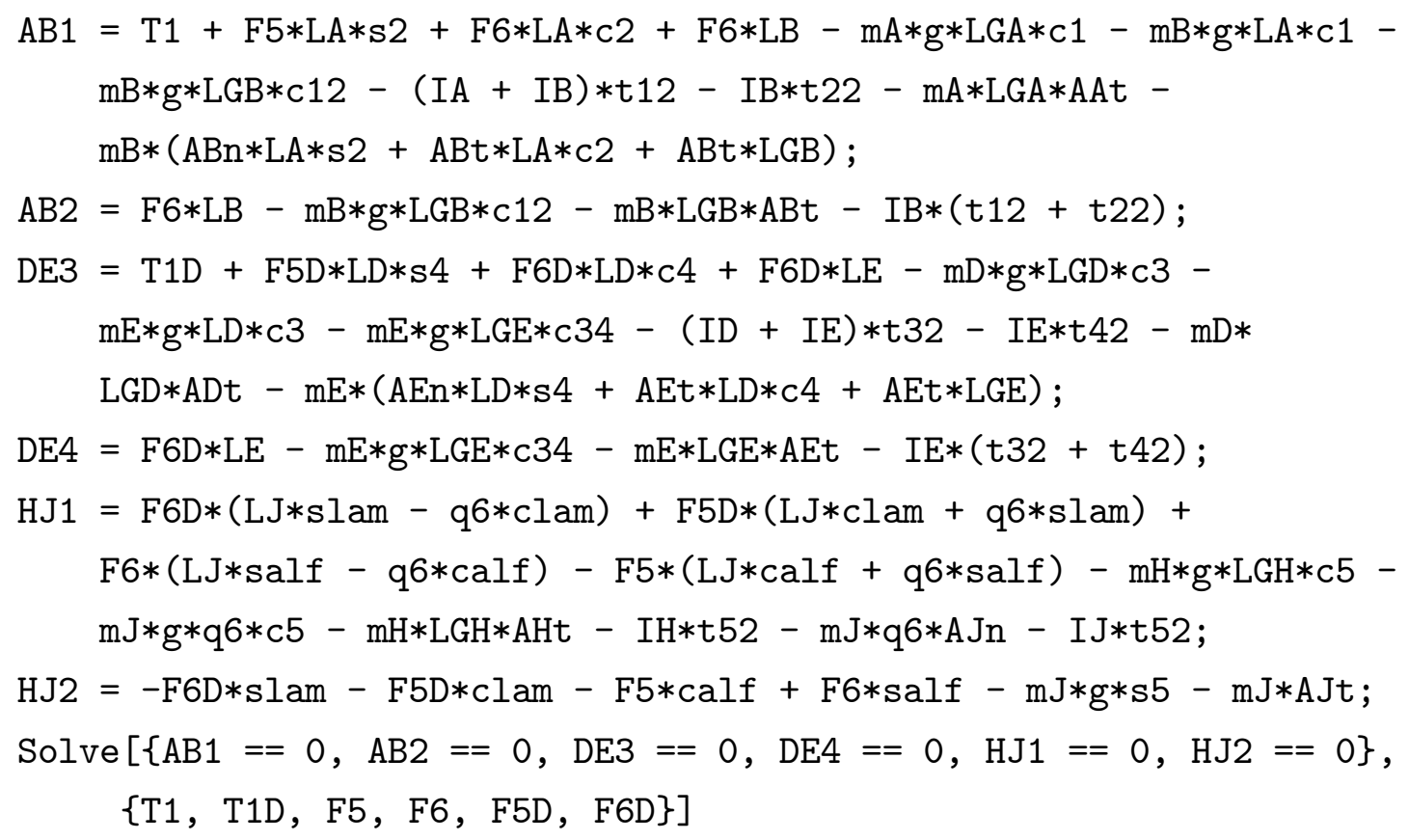




\section{Anexo K: PROGRAMA EQUAÇÕES DINÂMICA 2}

Neste anexo apresentamos o programa Mathematica que deduz as equações das componentes de reação $F_{1}$ e $F_{2}$ no atuador $O_{6}$, e das componentes $F_{3}$ e $F_{4}$ que atuam na junta $A$. Para isto utilizamos as equações $8.4,8.5,8.8$ e a quarta equação foi obtida multiplicando-se escalarmente a equação 8.2 pelo vetor unitário $\overrightarrow{a_{1}}$. Isto é:

$$
\begin{gathered}
F_{2} L_{G A}+F_{4} L_{G A}-m_{A} g L_{G A} c_{1}=m_{A} L_{G A} A_{A}^{t} \\
T_{1}-L_{G A} F_{2}+L_{A} F_{4}-L_{G A} F_{4}=I_{A} \dot{u}_{1} \\
F_{3} L_{G B} s_{2}-F_{4} L_{A}-F_{4} L_{G B} c_{2}+F_{5} L_{A} s_{2}+F_{6} L_{A} c_{2}+ \\
F_{6} L_{G B}-m_{B} g L_{A} c_{1}-m_{B} g L_{G B} c_{12} \\
=m_{B}\left(A_{B}^{n} L_{A} s_{2}+A_{B}^{t} L_{A} c_{2}+A_{B}^{t} L_{G B}\right)
\end{gathered}
$$

e

$$
F_{1}+F_{3}-m_{A} g s_{1}=m_{A} A_{A}^{n}
$$

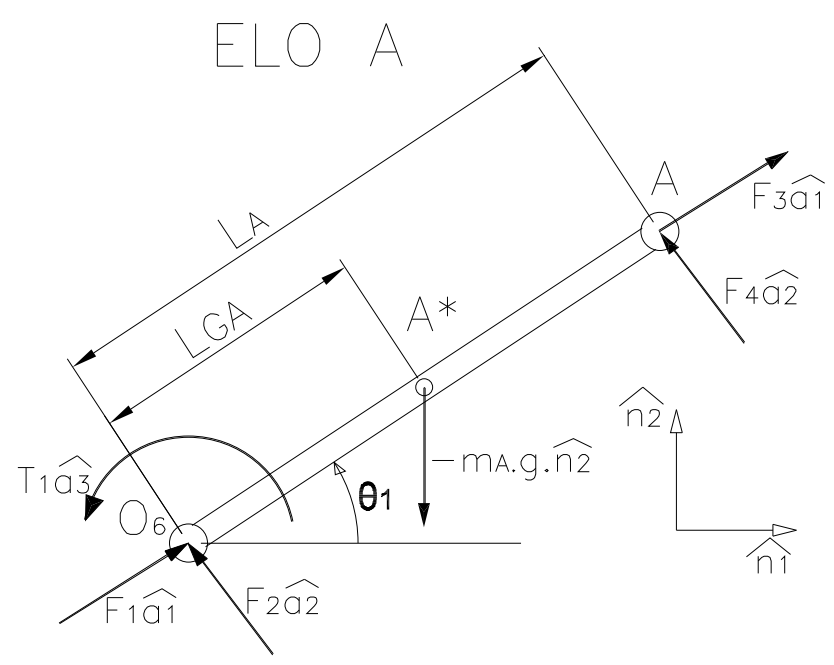

Figura 11.10: Anexo K: diagrama de corpo livre do elo A 


\section{LISTA DO PROGRAMA}

(*KANE2 : Determinacao das equacoes das forcas F1, F2, F3 e F4*)

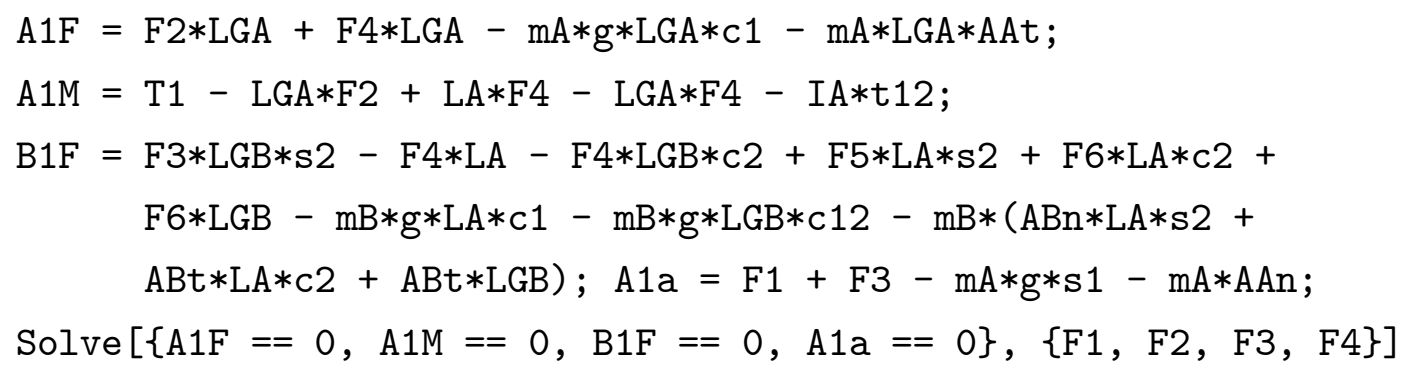




\section{Anexo L: PROGRAMA EQUAÇÕES DINÂMICA 3}

Neste anexo apresentamos o programa Mathematica que deduz as equações das componentes de reação no atuador $O_{2}$, isto é, $F_{1}^{D}$ e $F_{2}^{D}$ e das componentes $F_{3}^{D}$ e $F_{4}^{D}$ que atuam na junta $D$. Utilizamos as equações 8.16, 8.17, 8.20 e a quarta equação foi obtida multiplicando-se escalarmente a equação 8.14 pelo vetor unitário $\overrightarrow{d_{1}}$. Isto é:

$$
\begin{gathered}
F_{2}^{D} L_{G D}+F_{4}^{D} L_{G D}-m_{D} g L_{G D} c_{3}=m_{D} L_{G D} A_{D}^{t} \\
T_{1}^{D}-L_{G D} F_{2}^{D}+\left(L_{D}-L_{G D}\right) F_{4}^{D}=I_{D} \dot{u}_{3} \\
F_{3}^{D} L_{G E} s_{4}-F_{4}^{D} L_{D}-F_{4}^{D} L_{G E} c_{4}+F_{5}^{D} L_{D} s_{4}+F_{6}^{D} L_{D} c_{4}+ \\
F_{6}^{D} L_{G E}-m_{E} g L_{D} c_{3}-m_{E} g L_{G E} c_{34} \\
=m_{E}\left(A_{E}^{n} L_{D} s_{4}+A_{E}^{t} L_{D} c_{4}+A_{E}^{t} L_{G E}\right)
\end{gathered}
$$

e

$$
F_{1}^{D}+F_{3}^{D}-m_{D} g s_{3}=m_{D} A_{D}^{n}
$$

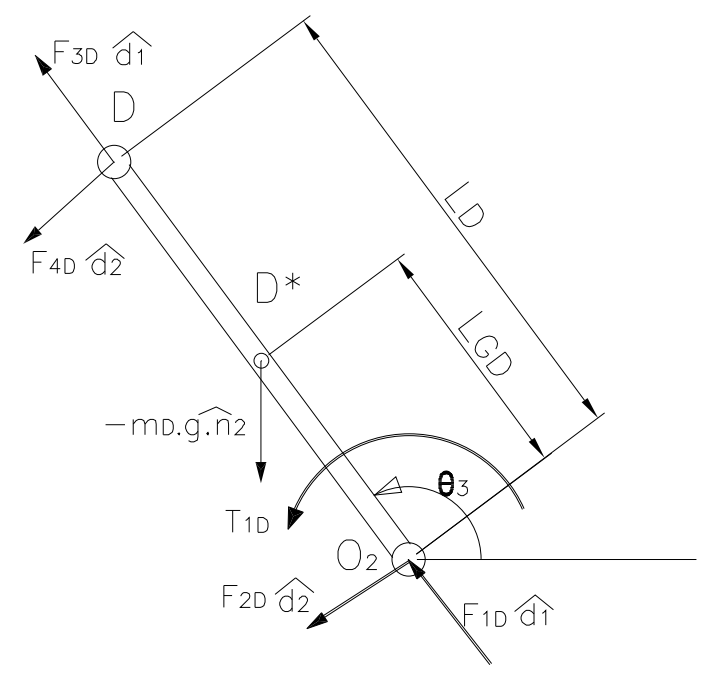

Figura 11.11: Anexo L: diagrama de corpo livre do elo D 


\section{LISTA DO PROGRAMA}

(*KANE3 : Determinacao das equacoes das forcas F1D, F2D, F3D e F4D *)

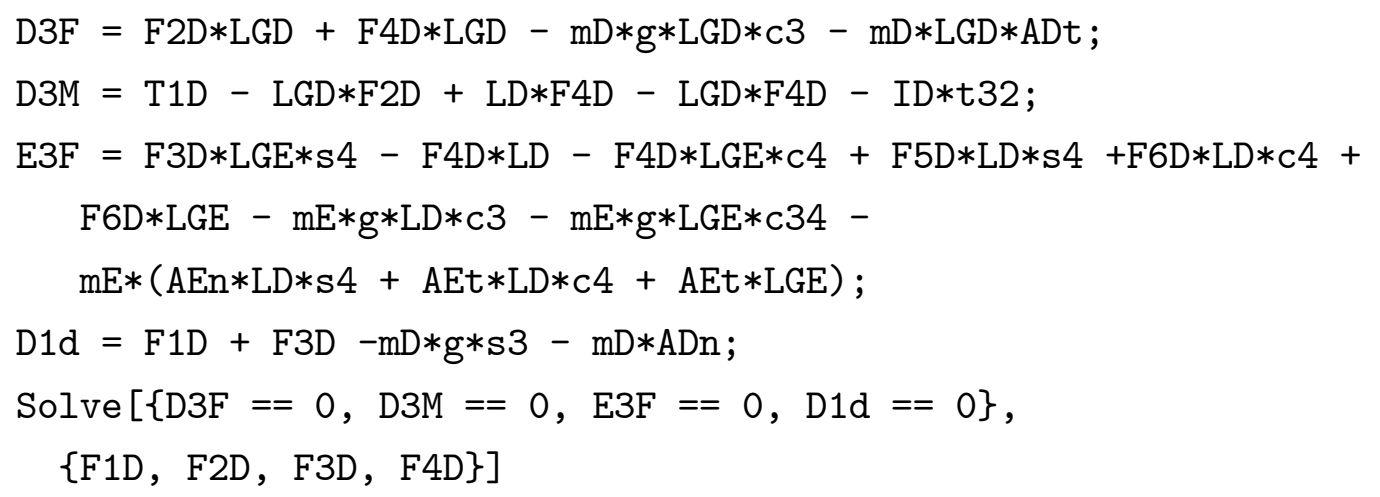




\section{Anexo M: PROGRAMA EQUAÇÕES DINÂMICA 4}

Neste anexo apresentamos o programa Mathematica que deduz as equações para a determinação das forças e torque de reação na cadeia passiva, isto é, $F_{1}^{H}$, $F_{2}^{H}, F_{3}^{H}$ e $T_{4}$. Utilizamos as seguintes equações:

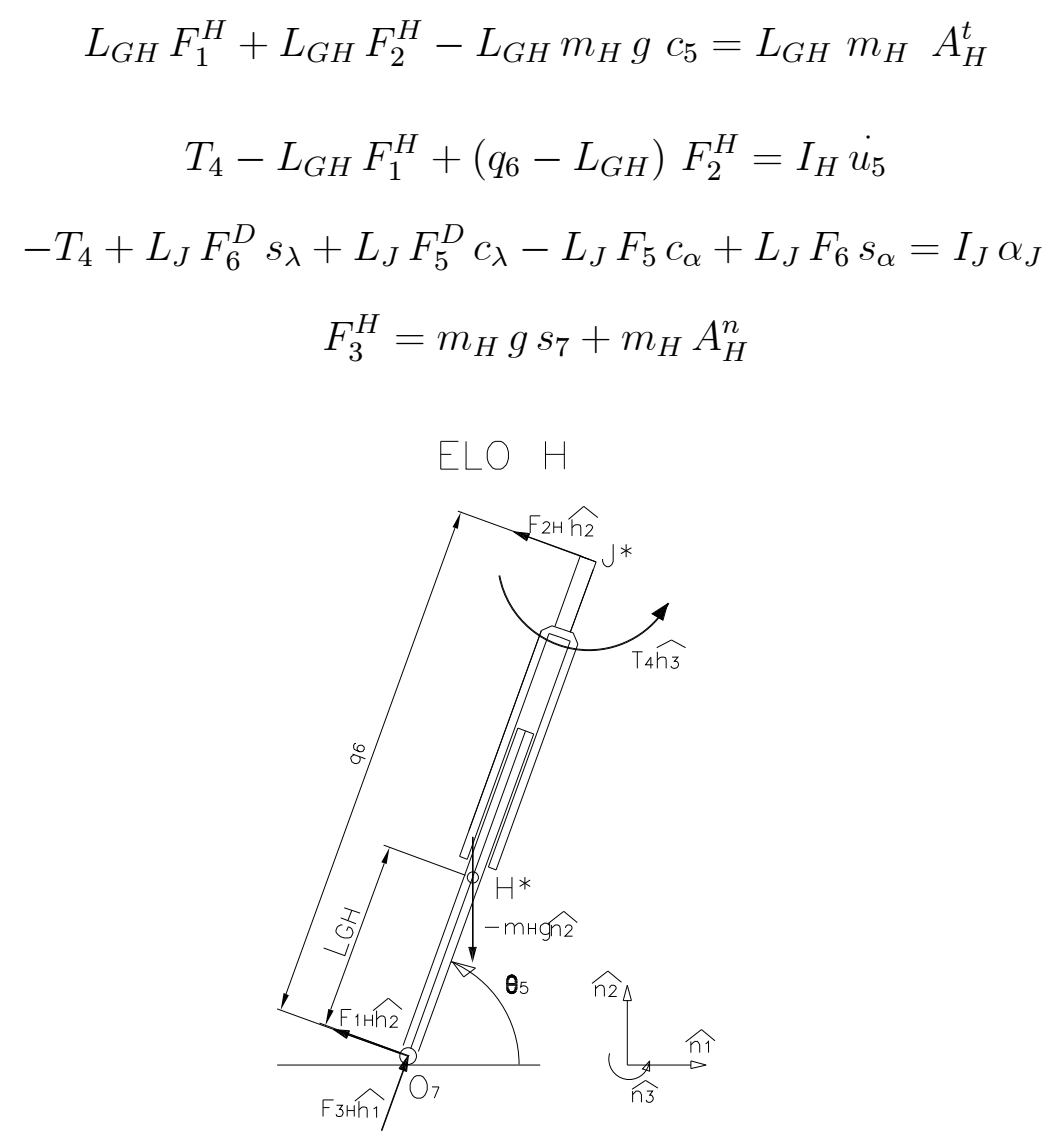

Figura 11.12: Anexo M: diagrama de corpo livre da cadeia passiva

\section{LISTA DO PROGRAMA}

(*KANE4 : Det. das equs forcas F1H, F2H F3H, e torque T4*) $\mathrm{H} 1 \mathrm{~F}=\mathrm{F} 1 \mathrm{H} * \mathrm{LGH}+\mathrm{F} 2 \mathrm{H} * \mathrm{LGH}-\mathrm{mH} * \mathrm{~g} * \mathrm{LGH} * \mathrm{c} 5-\mathrm{mH} * \mathrm{LGH} * \mathrm{AHt} ;$

$\mathrm{H} 1 \mathrm{M}=\mathrm{T} 4-\mathrm{LGH} * \mathrm{~F} 1 \mathrm{H}+\mathrm{q} 6 * \mathrm{~F} 2 \mathrm{H}-\mathrm{LGH} * \mathrm{~F} 2 \mathrm{H}-\mathrm{IH} * \mathrm{t} 52 ;$

$\mathrm{H} 3 \mathrm{~F}=\mathrm{F} 3 \mathrm{H}-\mathrm{mH} * \mathrm{~g} * \mathrm{~s} 5-\mathrm{mH} * \mathrm{AHn} ;$

$\mathrm{J} 2=-\mathrm{T} 4+\mathrm{F} 6 \mathrm{D} * \mathrm{LJ} * \mathrm{slam}+\mathrm{F} 5 \mathrm{D} * \mathrm{LJ} * \mathrm{clam}-\mathrm{F} 5 * \mathrm{LJ} * \mathrm{calf}+$

$\mathrm{F} 6 * \mathrm{LJ} * \mathrm{salf}-\mathrm{IJ} * \mathrm{t} 52$;

Solve $[\{\mathrm{H} 1 \mathrm{~F}==0, \mathrm{H} 1 \mathrm{M}==0, \mathrm{H} 3 \mathrm{~F}==0, \mathrm{~J} 2==0\},\{\mathrm{F} 1 \mathrm{H}, \mathrm{F} 2 \mathrm{H}, \mathrm{F} 3 \mathrm{H}, \mathrm{T} 4\}]$ 


\section{Anexo N: PROGRAMA DOS TORQUES DINÂMICOS DE REAÇÃO}

Este programa utiliza as equações deduzidas no Anexo J e calcula os valores numéricos dos torques de reação $T_{1}$ e $T_{1}^{D}$.

Neste programa devemos entrar com os seguintes dados:

-Ângulos dos elos: $\theta_{1}, \theta_{2}, \theta_{3}, \theta_{4}$ e $\theta_{5}$. (Sentido anti-horário é positivo)

-Comprimento dos elos: $L_{A}, L_{B}, L_{D}, L_{E}, L_{J}$, e $q_{6}$.

-Distâncias dos CG: $L_{G A}, L_{G B}, L_{G D}, L_{G E}$ e $L_{G H}$.

-Componentes da velocidade da garra: $V_{J x}$ e $V_{J y}$

-Componentes da aceleração da garra: $a_{J x}$ e $a_{J y}$.

-Aceleração da gravidade: $g$.

-Massas dos elos: $m_{A}, m_{B}, m_{D}, m_{E}, m_{H}$ e $m_{J}$.

-Momento de inércia dos elos: $I_{A}, I_{B}, I_{D}, I_{E}, I_{H}$ e $I_{J}$.

-Acelerações angulares: $\ddot{\theta}_{1}, \ddot{\theta}_{2}, \ddot{\theta}_{3}, \ddot{\theta}_{4}$ e $\ddot{\theta}_{5}$.

-Acelerações dos CG dos elos calculadas no anexo $\mathrm{H}: A_{A}^{n}, A_{A}^{t}, A_{B}^{n}, A_{B}^{t}, A_{D}^{n}$, $A_{D}^{t}, A_{E}^{n}, A_{E}^{t}, A_{H}^{n}, A_{H}^{t}, A_{J}^{n}$ e $A_{J}^{t}$.

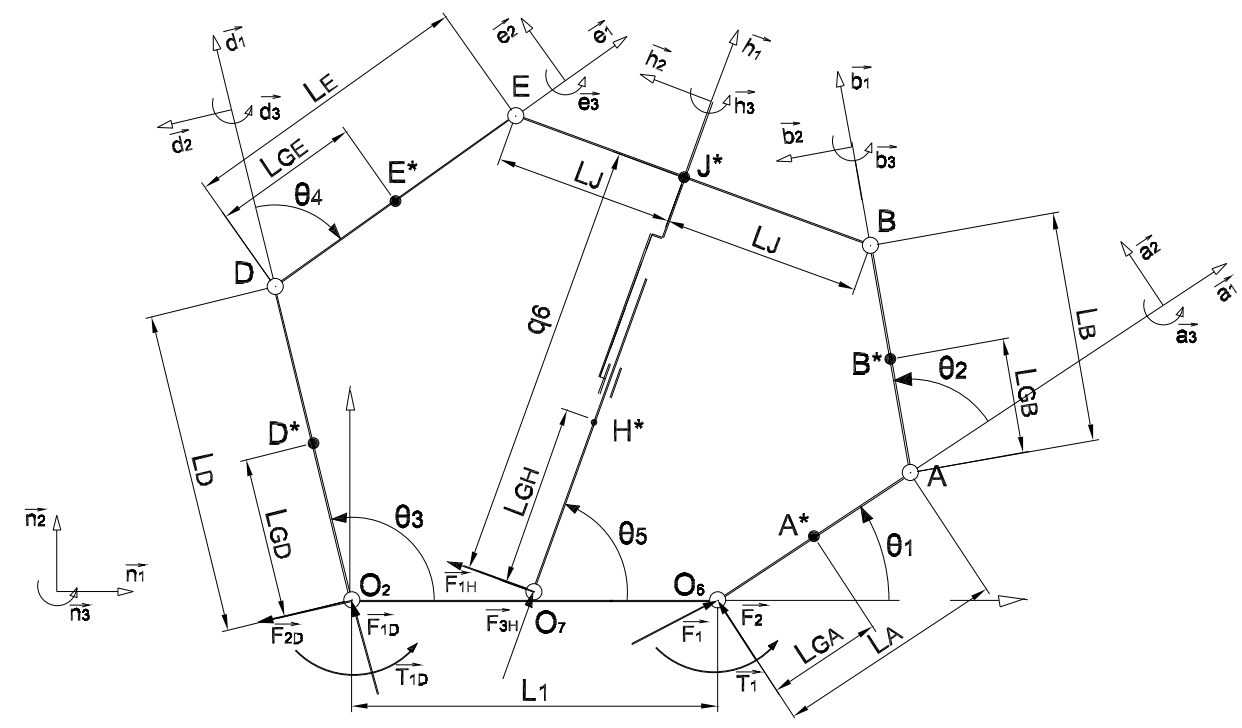

Figura 11.13: Anexo N: diagrama cinemático 
LISTA DO PROGRAMA

(*ANEXO N : KANE1a. Calculo dos torques de reacao T1 e T1D*)

(* Retorna : $\{\mathrm{T} 1 \mathrm{D}, \mathrm{T} 1\} *$ )

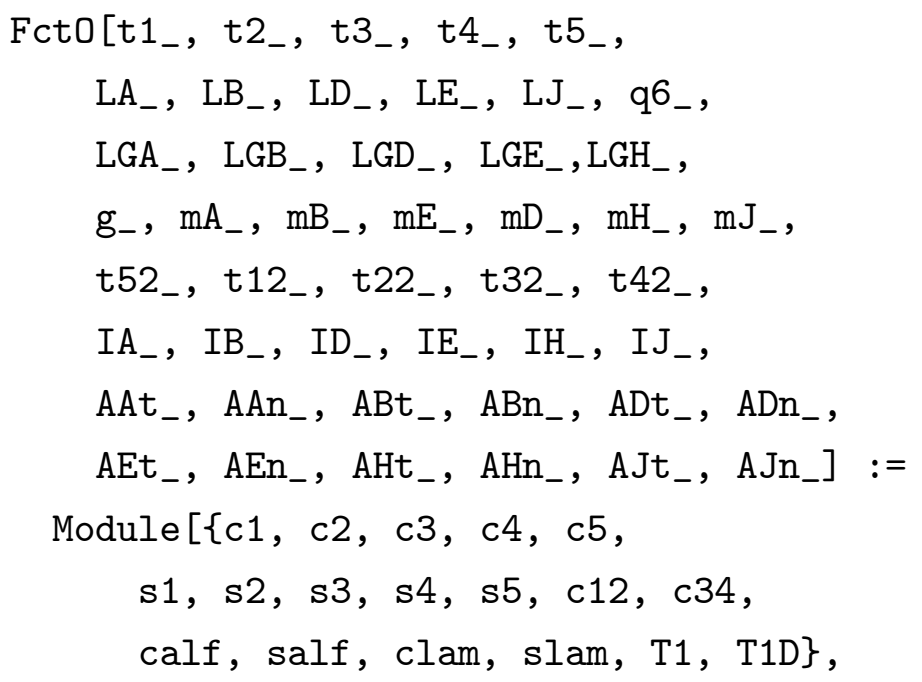

$c 1=\operatorname{Cos}[t 1] ; c 2=\operatorname{Cos}[t 2] ; c 3=\operatorname{Cos}[t 3] ; c 4=\operatorname{Cos}[t 4] ;$

$\mathrm{c} 5=\operatorname{Cos}[t 5] ; \mathrm{s} 1=\operatorname{Sin}[\mathrm{t} 1] ; \mathrm{s} 2=\operatorname{Sin}[\mathrm{t} 2] ; \mathrm{s} 3=\operatorname{Sin}[\mathrm{t} 3] ;$

$\mathrm{s} 4=\operatorname{Sin}[\mathrm{t} 4] ; \mathrm{s} 5=\operatorname{Sin}[\mathrm{t} 5] ; \mathrm{c} 12=\operatorname{Cos}[\mathrm{t} 1+\mathrm{t} 2]$;

c34 $=\operatorname{Cos}[t 3+t 4] ;$ calf $=\operatorname{Cos}[t 1+t 2-t 5]$;

salf $=\operatorname{Sin}[t 1+t 2-t 5] ; \operatorname{clam}=\operatorname{Cos}[t 5-t 4-t 3] ;$

slam $=$ Sin $[\mathrm{t5}-\mathrm{t} 4-\mathrm{t} 3]$;

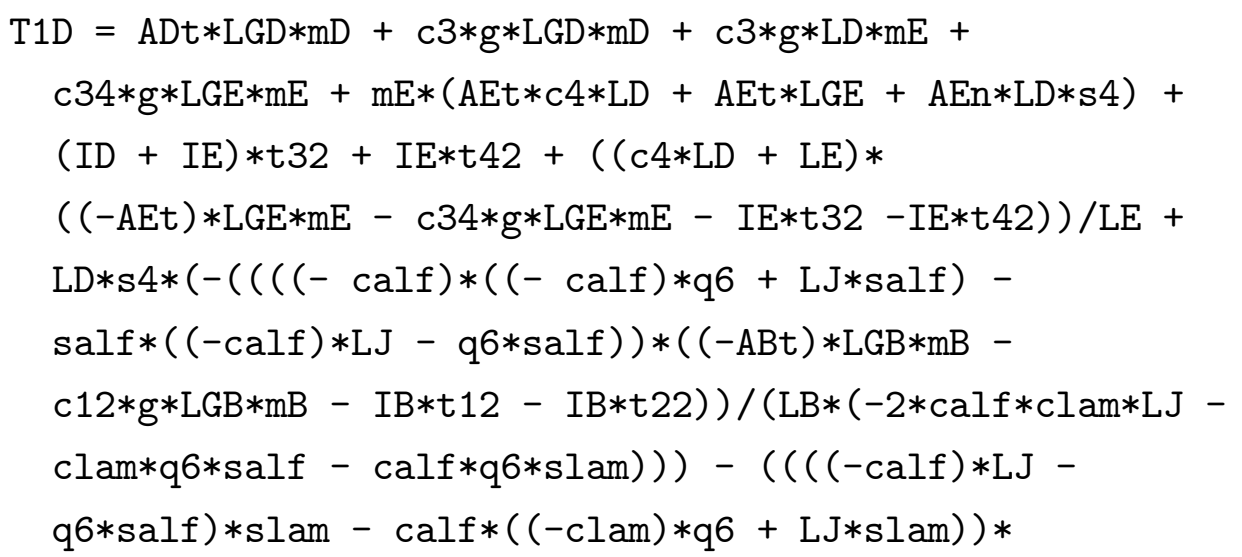




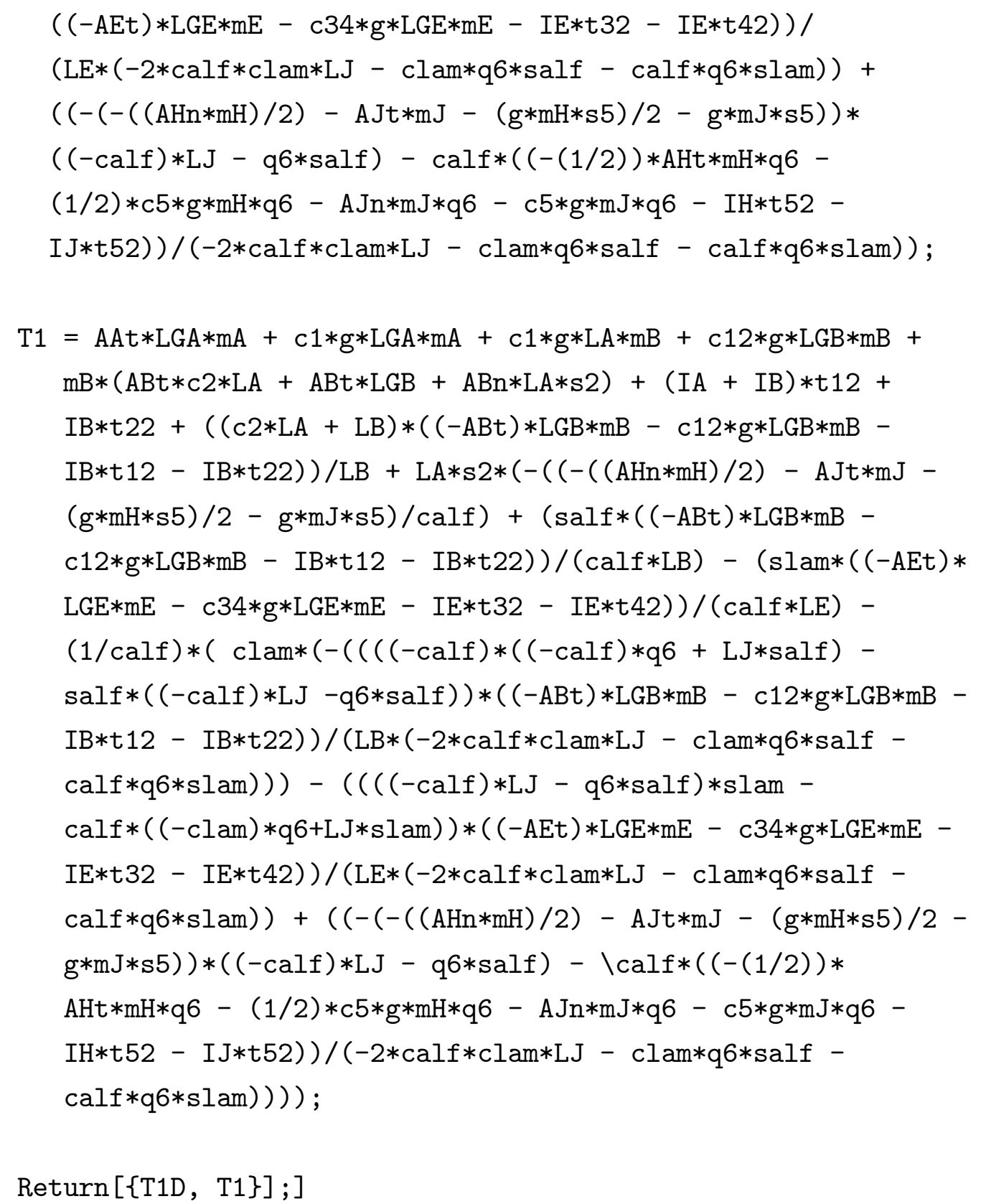




\section{Anexo O: PROGRAMA DAS FORÇAS DINÂMICAS NAS CADEIAS ATIVAS}

O programa deste anexo utiliza as equações deduzidas nos Anexos K e L e calcula as reações de apoio $F_{1}, F_{2}, F_{1}^{D}$ e $F_{2}^{D}$ e as forças $F_{3}, F_{4}, F_{5}, F_{6}, F_{3}^{D}, F_{4}^{D}$, $F_{5}^{D}$ e $F_{6}^{D}$, que atuam nas juntas.

Neste programa, além dos dados de entrada vistos no Anexo N devemos entrar também com os torques de reação $T_{1}$ e $T_{1}^{D}$ calculados neste mesmo anexo.

Portanto devemos entrar com os seguintes dados:

-Ângulos dos elos: $\theta_{1}, \theta_{2}, \theta_{3}, \theta_{4}$ e $\theta_{5}$. (Sentido anti-horário é positivo)

-Comprimento dos elos: $L_{A}, L_{B}, L_{D}, L_{E}, L_{J}$, e $q_{6}$.

-Distâncias dos CG: $L_{G A}, L_{G B}, L_{G D}, L_{G E}$ e $L_{G H}$.

-Componentes da velocidade da garra: $V_{J x}$ e $V_{J y}$

-Componentes da aceleração da garra: $a_{J x}$ e $a_{J y}$.

-Aceleração da gravidade: $g$.

-Massas dos elos: $m_{A}, m_{B}, m_{D}, m_{E}, m_{H}$ e $m_{J}$.

-Momento de inércia dos elos: $I_{A}, I_{B}, I_{D}, I_{E}, I_{H}$ e $I_{J}$.

-Acelerações angulares: $\ddot{\theta_{1}}, \ddot{\theta_{2}}, \ddot{\theta_{3}}, \ddot{\theta}_{4}$ e $\ddot{\theta}_{5}$

-Acelerações dos CG dos elos calculadas no anexo $\mathrm{H}: A_{A}^{n}, A_{A}^{t}, A_{B}^{n}, A_{B}^{t}, A_{D}^{n}$, $A_{D}^{t}, A_{E}^{n}, A_{E}^{t}, A_{H}^{n}, A_{H}^{t}, A_{J}^{n}$ e $A_{J}^{t}$.

-Torques de reação $T_{1}$ e $T_{1}^{D}$

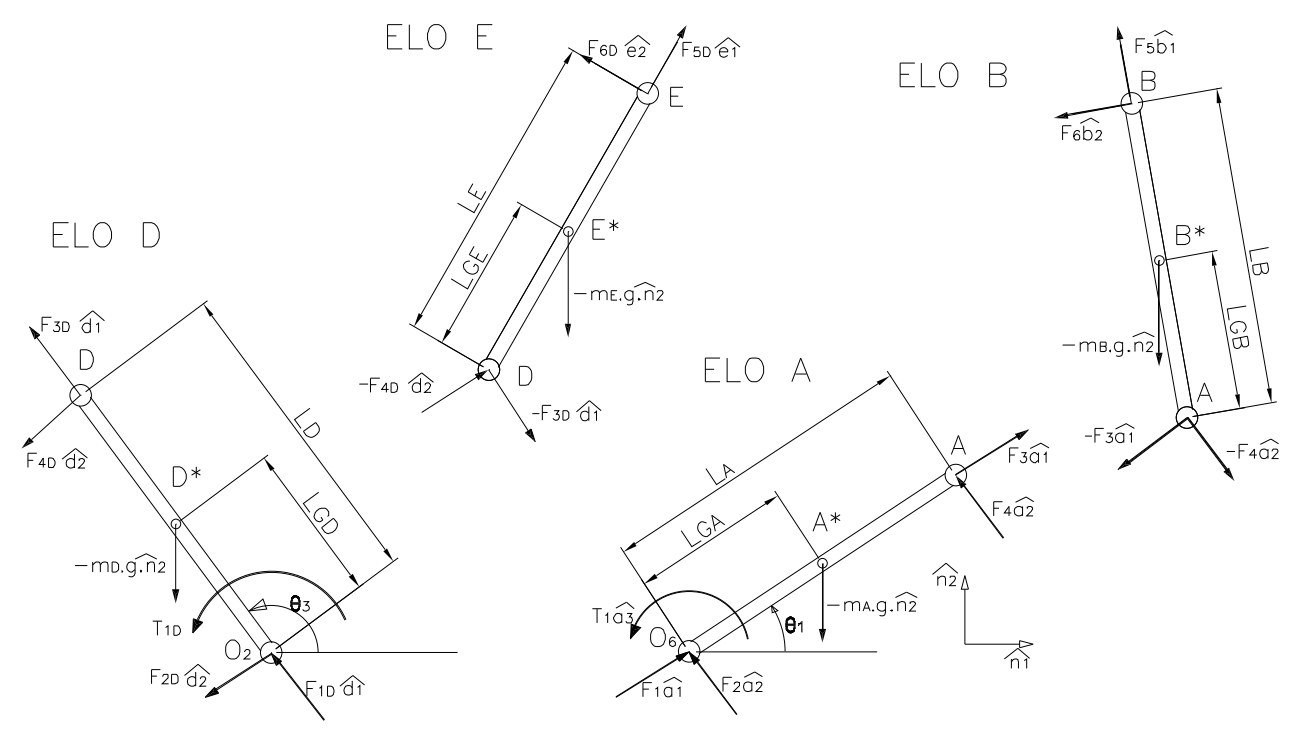

Figura 11.14: Anexo O: diagramas de corpo livre 


\section{LISTA DO PROGRAMA}

(*ANEXO 0 : KANE2a e 3a. Calculo das forcas F1 a F6, F1D a $\mathrm{F} 6 \mathrm{D} *)$

(* Resultado : \{F1, F1D, F2, F2D, F3, F3D, F4, F4D, F5, F5D, $\mathrm{F} 6, \mathrm{~F} 6 \mathrm{D}\} *$ )
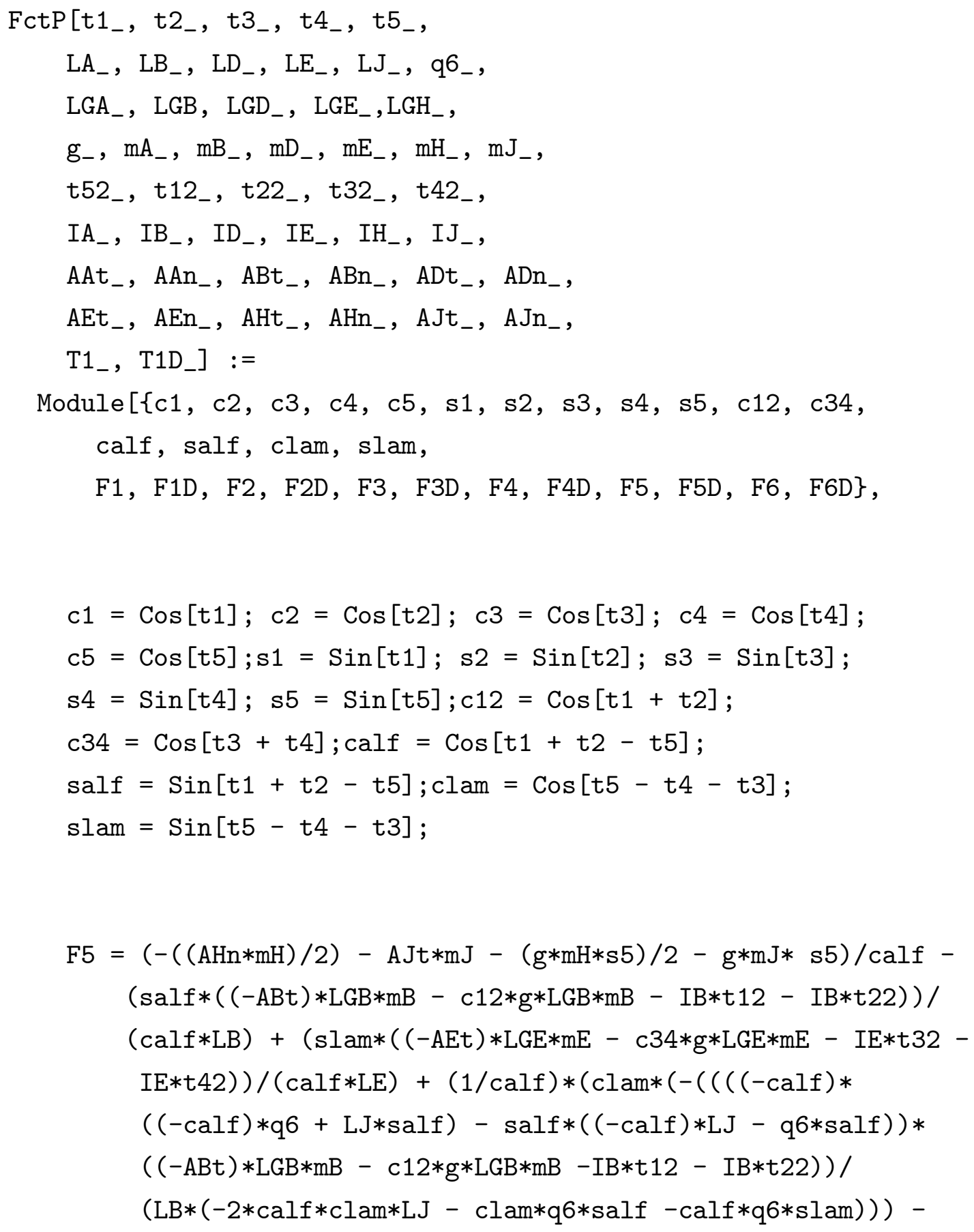


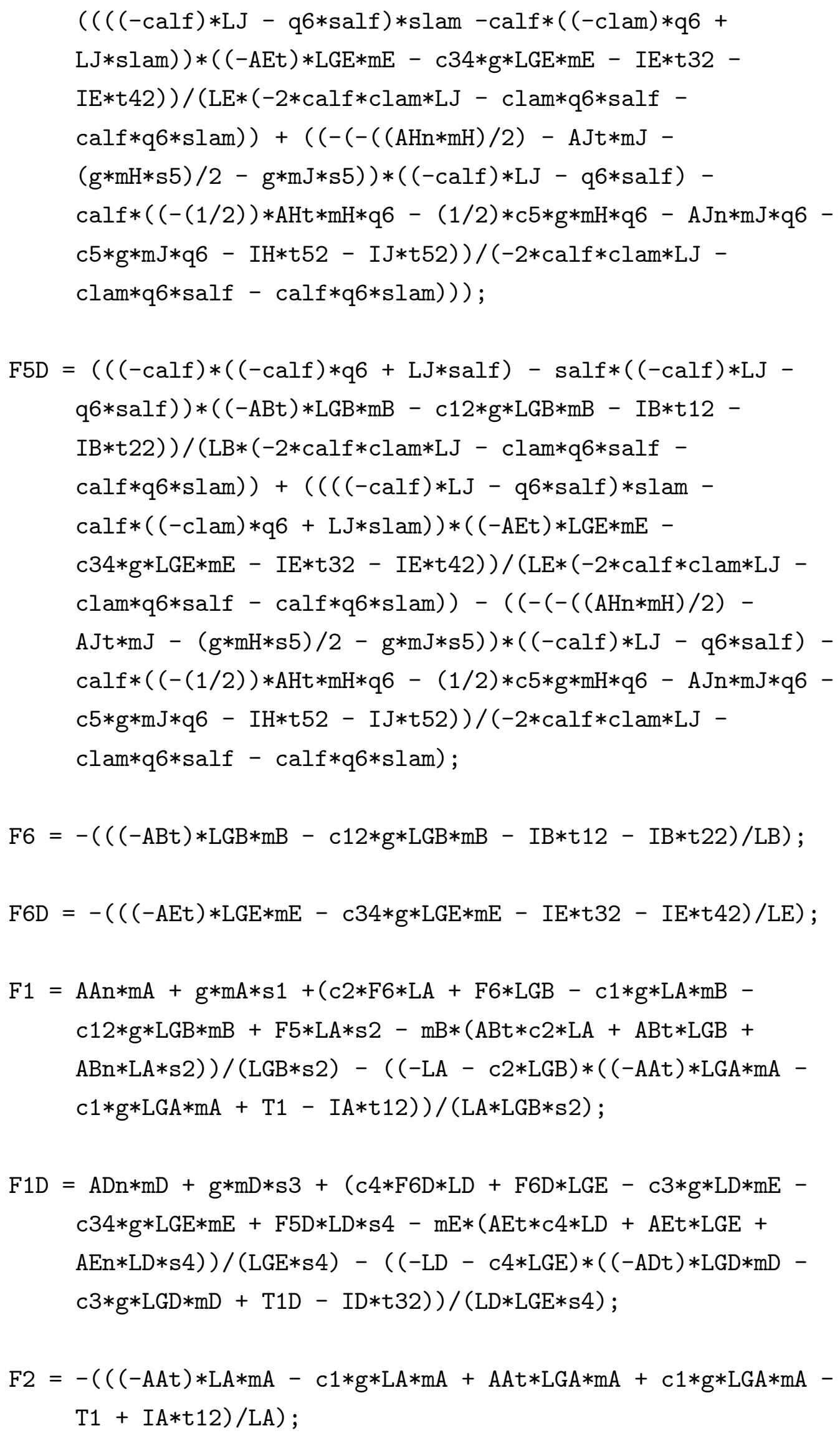




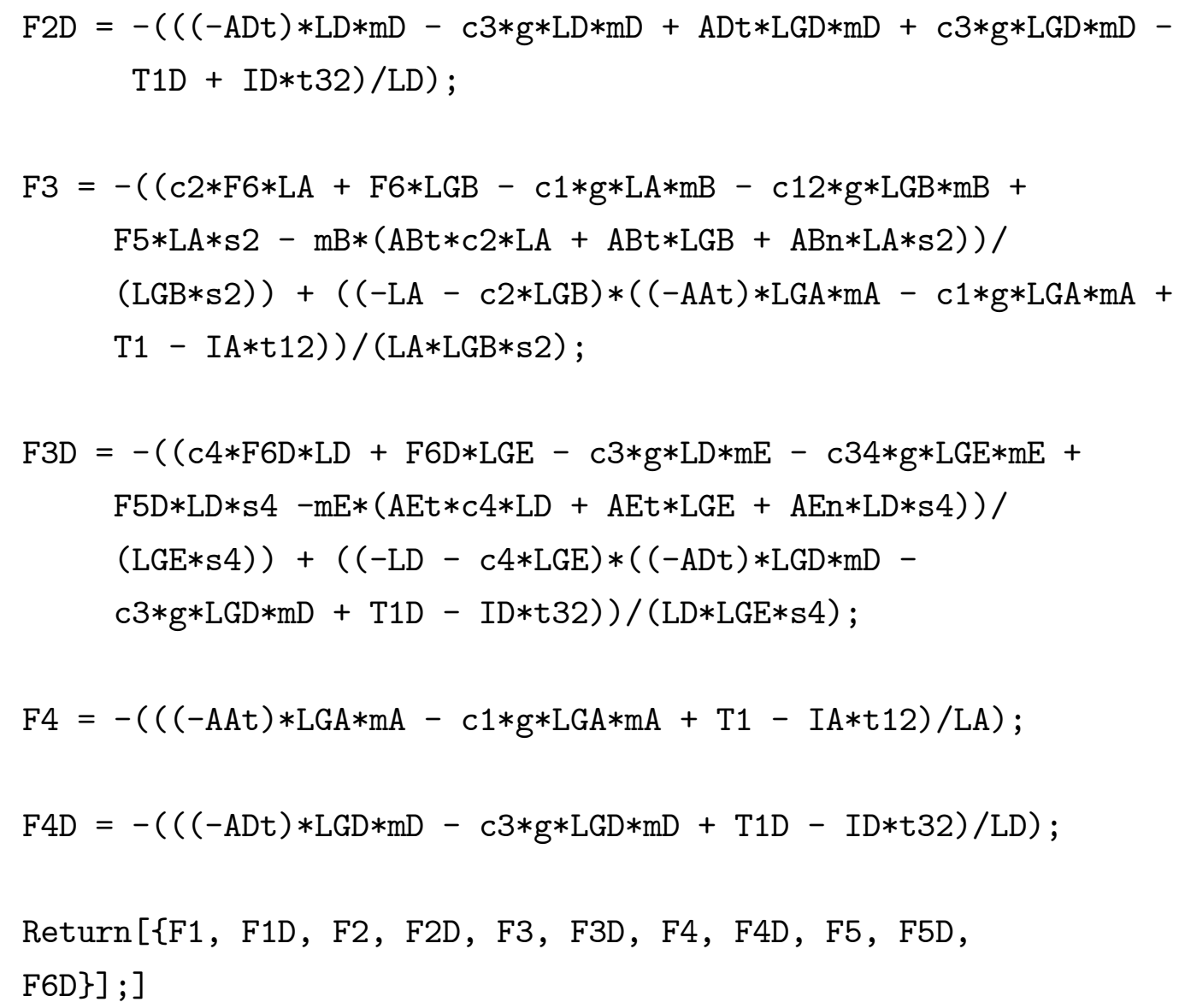




\section{Anexo P: PROGRAMA DAS FORÇAS E TORQUE DINÂMICOS NA CADEIA PASSIVA}

O programa deste anexo utiliza as equações deduzidas no Anexo M e calcula as forças $F_{1}^{H}, F_{2}^{H}, F_{3}^{H}$ e o torque $T_{4}$.

Neste programa, além dos dados de entrada vistos no Anexo O devemos entrar também com as forças $F_{5}, F_{6}, F_{5}^{D}$ e $F_{6}^{D}$, calculadas neste mesmo anexo.

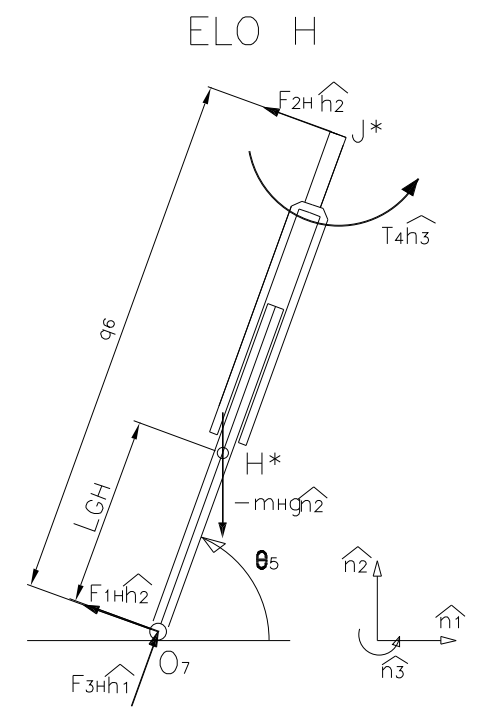

Figura 11.15: Anexo P: diagrama de corpo livre da cadeia passiva

\section{LISTA DO PROGRAMA}

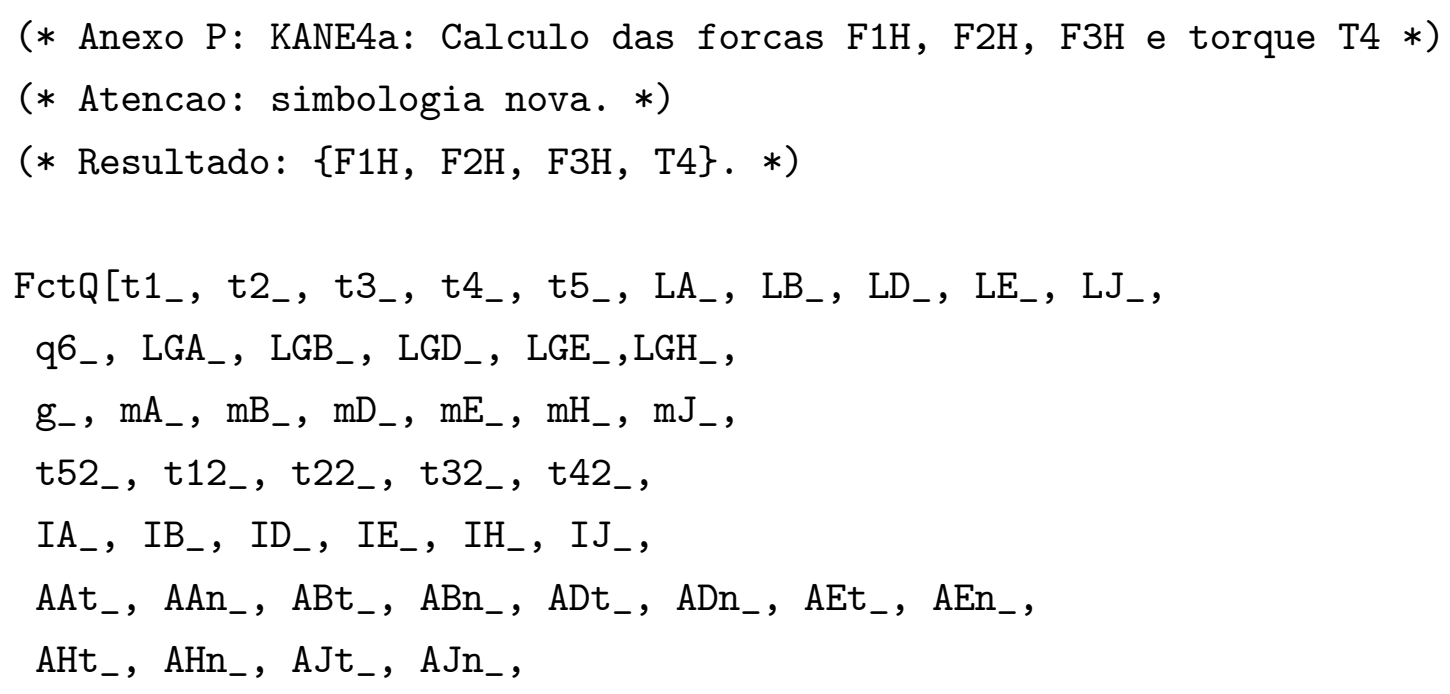




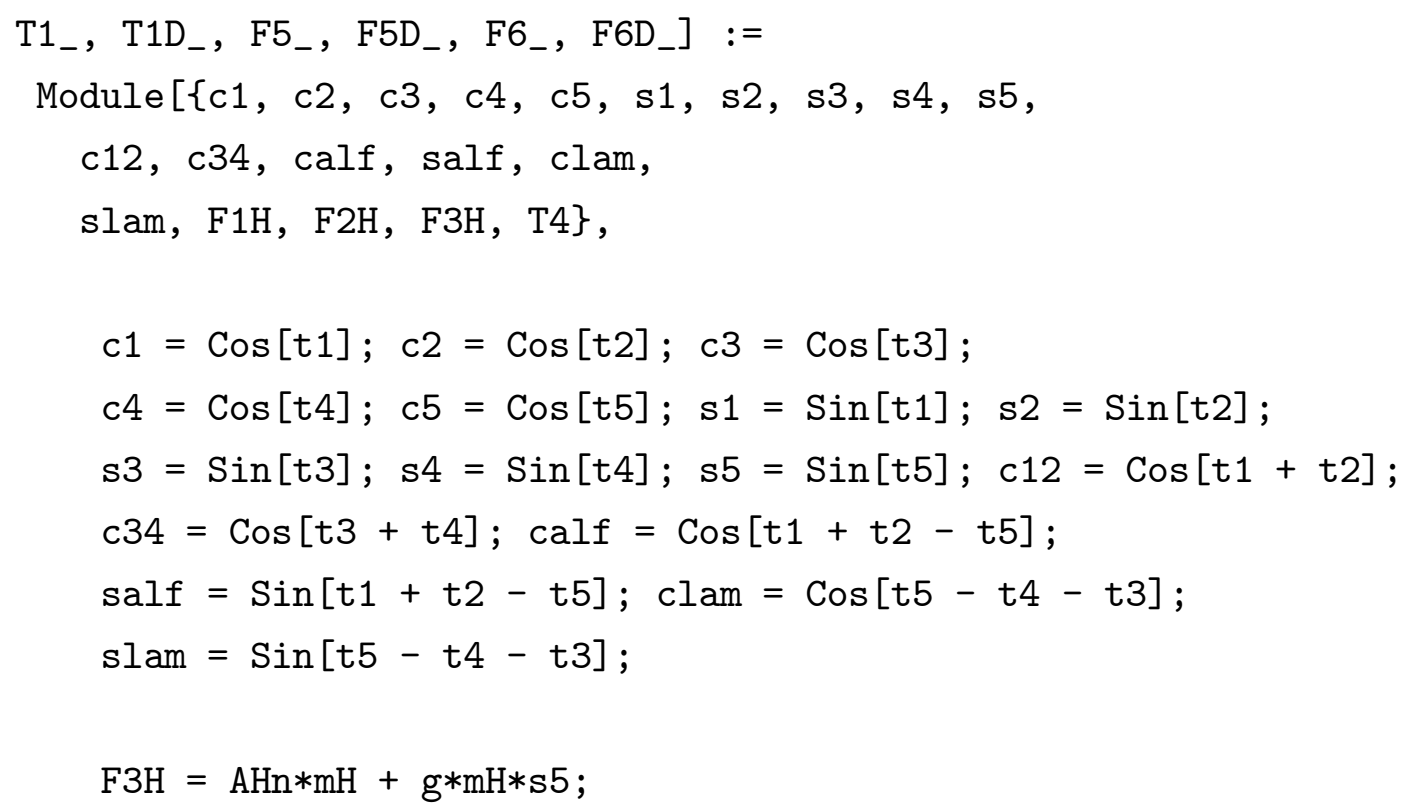

$\mathrm{F} 1 \mathrm{H}=-((1 /(2 * \mathrm{q} 6)) *(2 * \mathrm{calf} * \mathrm{~F} 5 * \mathrm{LJ}-2 * \mathrm{clam} * \mathrm{~F} 5 \mathrm{D} * \mathrm{LJ}-$ $\mathrm{AHt} * \mathrm{mH} * \mathrm{q} 6-\mathrm{c} 5 * \mathrm{~g} * \mathrm{mH} * \mathrm{q} 6-2 * \mathrm{~F} 6 * \mathrm{LJ} * \mathrm{salf}-2 * \mathrm{~F} 6 \mathrm{D} * \mathrm{LJ} * \mathrm{slam}+$ $2 * \mathrm{IH} * \mathrm{t} 52+2 * \mathrm{IJ} * \mathrm{t} 52))$;

$\mathrm{F} 2 \mathrm{H}=-((1 /(2 * \mathrm{q} 6)) *(-2 * \mathrm{cal} f * \mathrm{~F} 5 * \mathrm{LJ}+2 * \mathrm{clam} * \mathrm{~F} 5 \mathrm{D} * \mathrm{LJ}-$ $\mathrm{AHt} * \mathrm{mH} * \mathrm{q} 6-\mathrm{c} 5 * \mathrm{~g} * \mathrm{mH} * \mathrm{q} 6+2 * \mathrm{~F} 6 * \mathrm{LJ} * \mathrm{salf}+2 * \mathrm{~F} 6 \mathrm{D} * \mathrm{LJ} * \mathrm{slam}-$ $2 * I H * t 52-2 * I J * t 52))$;

$\mathrm{T} 4=(-\mathrm{calf}) * \mathrm{~F} 5 * \mathrm{LJ}+\mathrm{clam} * \mathrm{~F} 5 \mathrm{D} * \mathrm{LJ}+\mathrm{F} 6 * \mathrm{LJ} * \mathrm{salf}+\mathrm{F} 6 \mathrm{D} * \mathrm{LJ} * \mathrm{slam}-$ IJ*t52;

$\operatorname{Return}[\{\mathrm{F} 1 \mathrm{H}, \mathrm{F} 2 \mathrm{H}, \mathrm{F} 3 \mathrm{H}, \mathrm{T} 4\}] ;]$ 


\section{Anexo Q: PROGRAMA TOTAL DOS TORQUES E FORÇAS DE REAÇÃO}

Este programa aglutina os programas anteriores e calcula diretamente os torques de reação nos atuadores além de outros esforços.

Os seguintes dados de entrada são necessários:

-Comprimentos dos elos $L_{1}, L_{A}, L_{B}, L_{D}, L_{E}$ e $L_{J}$.

-Posição dos CG nos elos: $L_{G A}, L_{G B}, L_{G D}, L_{G E}$ e $L_{G H}$.

-Posição da garra (ponto $J *$ ): $q_{6}$ e $\theta_{5}$.

-Componentes da velocidade do ponto $J *: V_{J}^{x}$ e $V_{J}^{y}$.

-Componentes da aceleração do ponto $J *: a_{J}^{x}$ e $a_{J}^{y}$.

-Aceleração da gravidade: $g$.

-Massas dos elos: $m_{A}, m_{B}, m_{D}, m_{E}, m_{H}$ e $m_{J}$.

-Momento de inércia dos elos: $I_{A}, I_{B}, I_{D}, I_{E}, I_{H}$ e $I_{J}$.

-Força externa aplicada na garra e seu ângulo de posição: $F$ e $\beta$.

-Torque externo aplicado na garra: $M$.

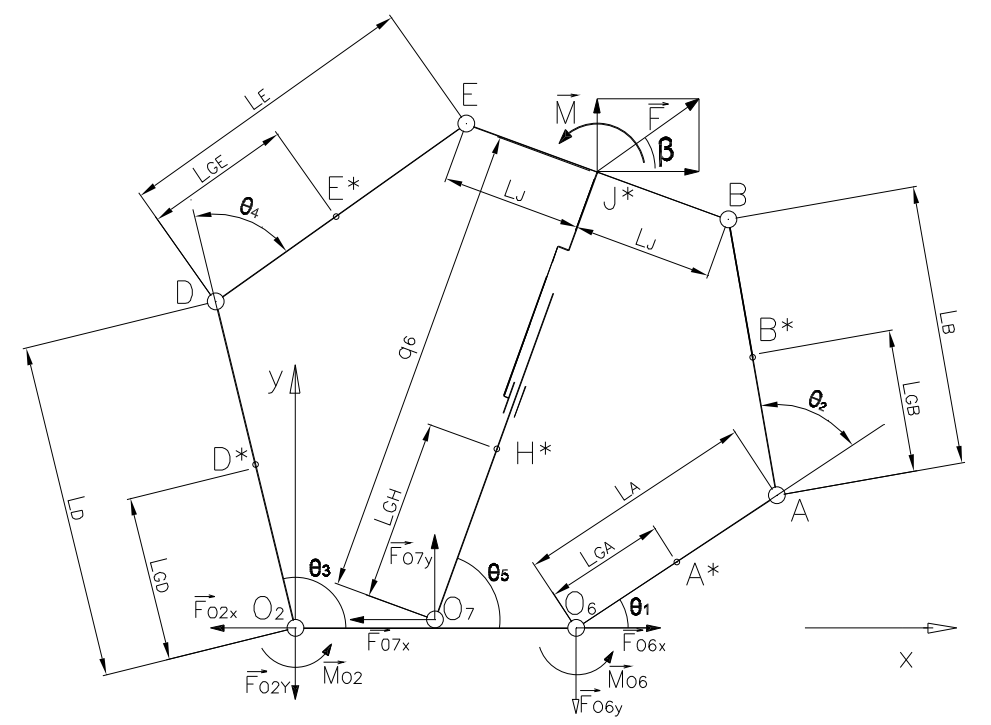

Figura 11.16: Anexo Q: diagrama cinemático

Este programa determina, entre outros esforços, os seguintes:

1- $\left(M_{2}, M_{8}\right)$ : Torques estáticos de reação respectivamente nos atuadores $\mathrm{O}_{2}$ e $O_{6}$ levando-se em conta somente os esforços na garra $(F$ e $M)$.

2- $\left(T_{1 D}, T_{1}\right)$ : Torques dinâmicos de reação respectivamente nos atuadores $O_{2}$ e $O_{6}$ levando-se em conta somente os efeito das massas dos elos. 
3- $\left(M_{O 2}, M_{O 6}\right)$ : Torques finais (soma dos torques anteriores).respectivamente nos atuadores $\mathrm{O}_{2}$ e $\mathrm{O}_{6}$.

\section{LISTA DO PROGRAMA}

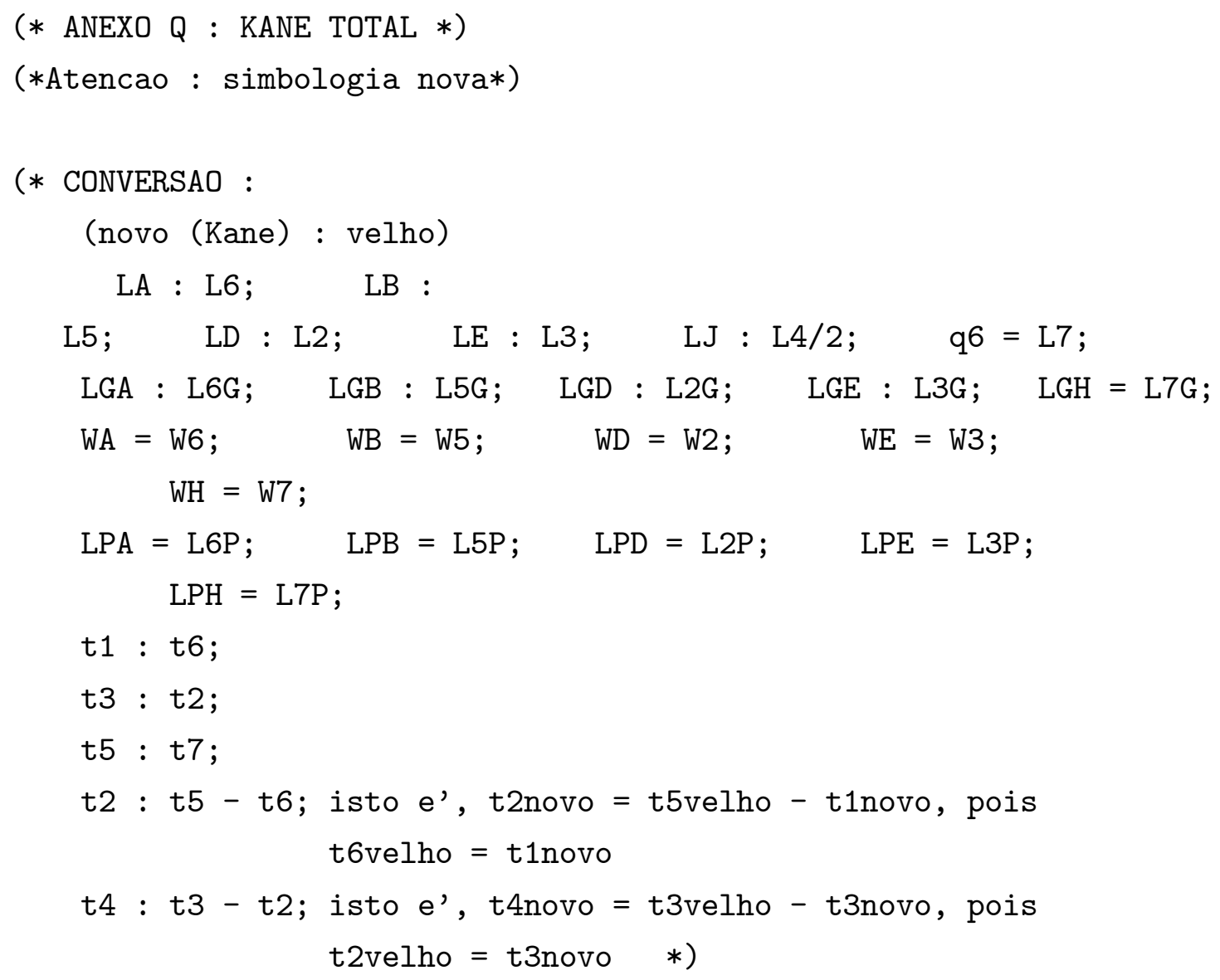


M02, MO6, F02x, F02y, F06x, F06y, tt4, tt2\},

$\mathrm{LPA}=0 ; \mathrm{LPB}=0 ; \mathrm{LPD}=0 ; \mathrm{LPE}=0 ; \mathrm{LPH}=0 ; \quad W A=0 ; W B=0 ;$

$\mathrm{WD}=0 ; \mathrm{WE}=0 ; \mathrm{WH}=0 ;$

$\{F 1 x, F 1 y, F 2 x, F 2 y, F 3 x, F 3 y, F F 4, F 5 x, F 5 y, F 6 x, F 6 y, F 7 x$,

$F 7 y, F 8 x, F 8 y, M 2, M 4, M 8, t 3, t t 4, t t 2, t 1$, sing, graf $\}=$ FctI2 [L1, LD, LE, $2 * \mathrm{LJ}, \mathrm{LB}, \mathrm{LA}$,

q6, t5,

LGD, LGE, LGB, LGA, LGH,

LPD, LPE, LPB, LPA, LPH,

$\mathrm{F}, \mathrm{b}, \mathrm{M}$,

$0,0,0,0,0,0$,

$\mathrm{WD}, \mathrm{WE}, \mathrm{WB}, \mathrm{WA}, \mathrm{WH}]$;

t4 $=$ tt4 - t3;

t2 $=$ tt2 - t1;

\{t11, t12, t21, t22, t31, t32, t41, t42, t51, t52, q61, q62,

$\mathrm{AAn}, \mathrm{AAt}, \mathrm{ABn}, \mathrm{ABt}, \mathrm{ADn}, \mathrm{ADt}, \mathrm{AEn}, \mathrm{AEt}, \mathrm{AHn}, \mathrm{AHt}$, $A J n, A J t\}=$

FctH $[t 1, t 2, t 3, t 4, t 5$,

LA, LB, LD, LE, LJ, q6,

LGA, LGB, LGD, LGE, LGH,

VJx, VJy, aJx, aJy] ;

$\{\mathrm{T} 1 \mathrm{D}, \mathrm{T} 1\}=$

Fct0 $[t 1, \mathrm{t} 2, \mathrm{t} 3, \mathrm{t} 4, \mathrm{t} 5$,

LA, LB, LD, LE, LJ, q6,

LGA, LGB, LGD, LGE, LGH,

$\mathrm{g}, \mathrm{mA}, \mathrm{mB}, \mathrm{mE}, \mathrm{mD}, \mathrm{mH}, \mathrm{mJ}$,

t52, t12, t22, t32, t42,

$I A, I B, I D, I E, I H, I J$,

AAt, $A A n, A B t, A B n, A D t, A D n, A E t$,

AEn, AHt, AHn, AJt, AJn];

$\{F 1, F 1 D, F 2, F 2 D, F 3, F 3 D, F 4, F 4 D, F 5, F 5 D, F 6, F 6 D\}=$ FctP $[t 1$, t2, t3, t4, t5,

LA, LB, LD, LE, LJ, q6, 
LGA, LGB, LGD, LGE, LGH,

$\mathrm{g}, \mathrm{mA}, \mathrm{mB}, \mathrm{mD}, \mathrm{mE}, \mathrm{mH}, \mathrm{mJ}$,

t52, t12, t22, t32, t42,

IA, IB, ID, IE, IH, IJ,

AAt, AAn, ABt, ABn, ADt, ADn,

AEt, AEn, AHt, AHn, AJt, AJn,

T1, T1D] ;

( $*$ resultados $*$ )

MO2 = M2 + T1D;

$\mathrm{MO} 6=\mathrm{M} 8+\mathrm{T} 1 ;$

$\mathrm{F} 02 \mathrm{x}=\mathrm{F} 2 \mathrm{D} * \operatorname{Cos}[\mathrm{t} 3+\mathrm{Pi} / 2]+\mathrm{F} 1 \mathrm{D} * \operatorname{Cos}[\mathrm{t} 3]+\mathrm{F} 1 \mathrm{x} ;$

$\mathrm{F} 02 \mathrm{y}=\mathrm{F} 2 \mathrm{D} * \mathrm{Sin}[\mathrm{t} 3+\mathrm{Pi} / 2]+\mathrm{F} 1 \mathrm{D} * \mathrm{Sin}[\mathrm{t} 3]+\mathrm{F} 1 \mathrm{y} ;$

$\mathrm{F} 06 \mathrm{x}=\mathrm{F} 2 * \operatorname{Cos}[\mathrm{t} 1+\mathrm{Pi} / 2]+\mathrm{F} 1 * \operatorname{Cos}[\mathrm{t} 1]+\mathrm{F} 8 \mathrm{x} ;$

$\mathrm{F} 06 \mathrm{y}=\mathrm{F} 2 * \operatorname{Sin}[\mathrm{t} 1+\mathrm{Pi} / 2]+\mathrm{F} 1 * \operatorname{Sin}[\mathrm{t} 1]+\mathrm{F} 8 \mathrm{y} ;$

(* calcular F02x, F02y, F06x, F06y *)

Return [\{M02, M06, F02x, F02y, F06x, F06y,

F1x, F1y, F2x, F2y, F3x, F3y, FF4, F5x, F5y, F6x, F6y, F7x, F7y, F8x, F8y, M2, M4, M8, t3, t4, t2, t1, sing, graf, t11, t12, t21, t22, t31, t32, t41, t42, t51, t52, q61, q62, AAn, AAt, ABn, ABt, ADn, ADt, AEn, AEt, AHn, AHt, AJn, AJt, T1D, T1,F1, F1D, F2, F2D, F3, F3D, F4, F4D, F5, F5D, $\mathrm{F} 6, \mathrm{~F} 6 \mathrm{D}\}] ;]$ 


\section{Anexo R: PROGRAMA EQUAÇÕES DINÂMICA 5}

Neste anexo apresentamos o programa Mathematica que deduz as equações dos torques de reação $T_{1}$ e $T_{1}^{D}$ considerando-se a atuação da força de atrito $\left(F_{a}\right)$ na junta prismática, da força $(F)$ e torque $(M)$ na garra.

\section{LISTA DO PROGRAMA}

(* Eqs dos torques T1, T1D, considerando e atrito na junta prismatica e esforcos na garra *)

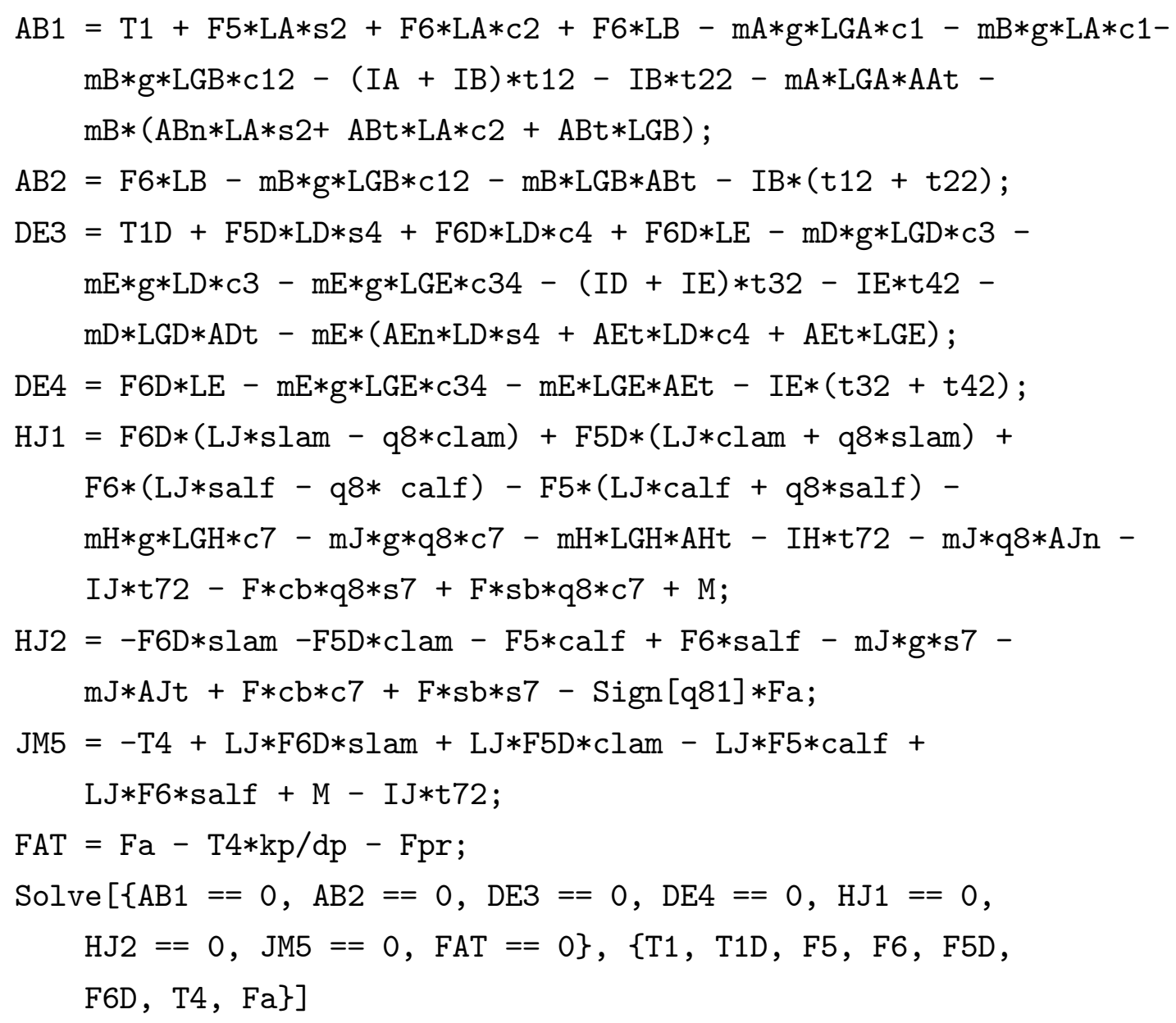




\section{Anexo S: PROGRAMA DOS TORQUES DE REAÇÃO COM ATRITO}

Este programa calcula diretamente os torques nos atuadores, levando em conta o atrito na junta prismática e os esforços na garra..

Os seguintes dados de entrada são necessários:

-Comprimentos dos elos $L_{1}, L_{A}, L_{B}, L_{D}, L_{E}$ e $L_{J}$.

-Posição dos CG nos elos: $L_{G A}, L_{G B}, L_{G D}, L_{G E}$ e $L_{G H}$.

-Posição da garra (ponto $J *$ ): $q_{6}$ e $\theta_{5}$.

-Componentes da velocidade do ponto $J *: V_{J}^{x}$ e $V_{J}^{y}$.

-Componentes da aceleração do ponto $J *: a_{J}^{x}$ e $a_{J}^{y}$.

-Aceleração da gravidade: $g$.

-Massas dos elos: $m_{A}, m_{B}, m_{D}, m_{E}, m_{H}$ e $m_{J}$.

-Momento de inércia dos elos: $I_{A}, I_{B}, I_{D}, I_{E}, I_{H}$ e $I_{J}$.

-Força externa aplicada na garra e seu ângulo de posição: $F$ e $\beta$.

-Torque externo aplicado na garra: $M$.

-Comprimento da junta prismática: $d_{p}$.

-Coeficiente de atrito na junta prismática: $k_{p}$.

-Força de atrito da proteção da junta prismática: $F_{p r}$.

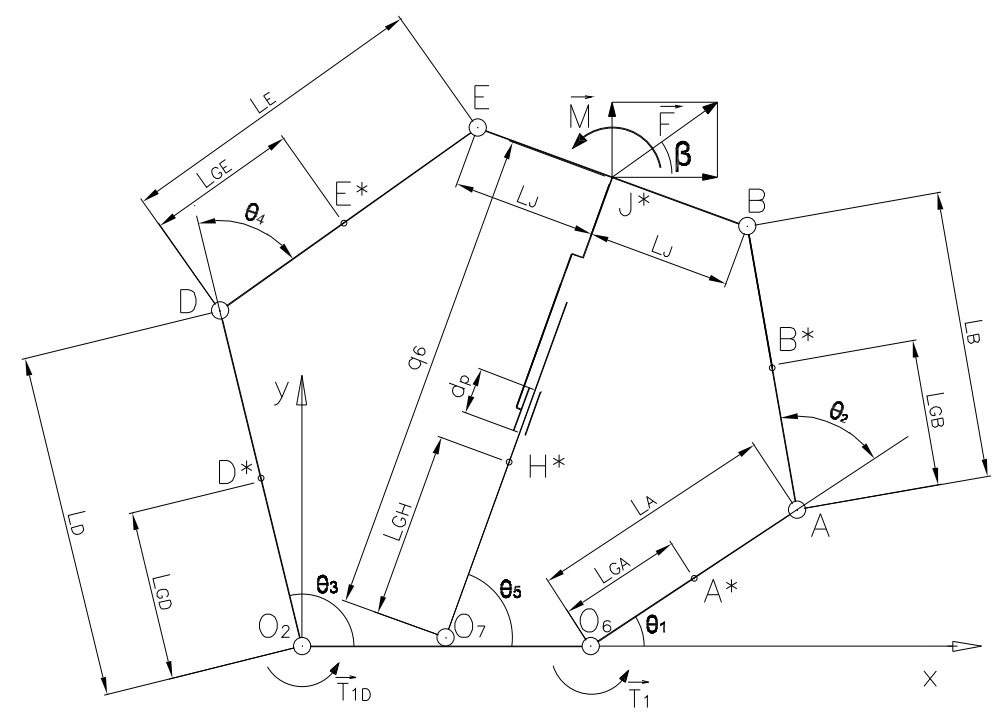

Figura 11.17: Anexo S: diagrama cinemático 


\section{LISTA DO PROGRAMA}

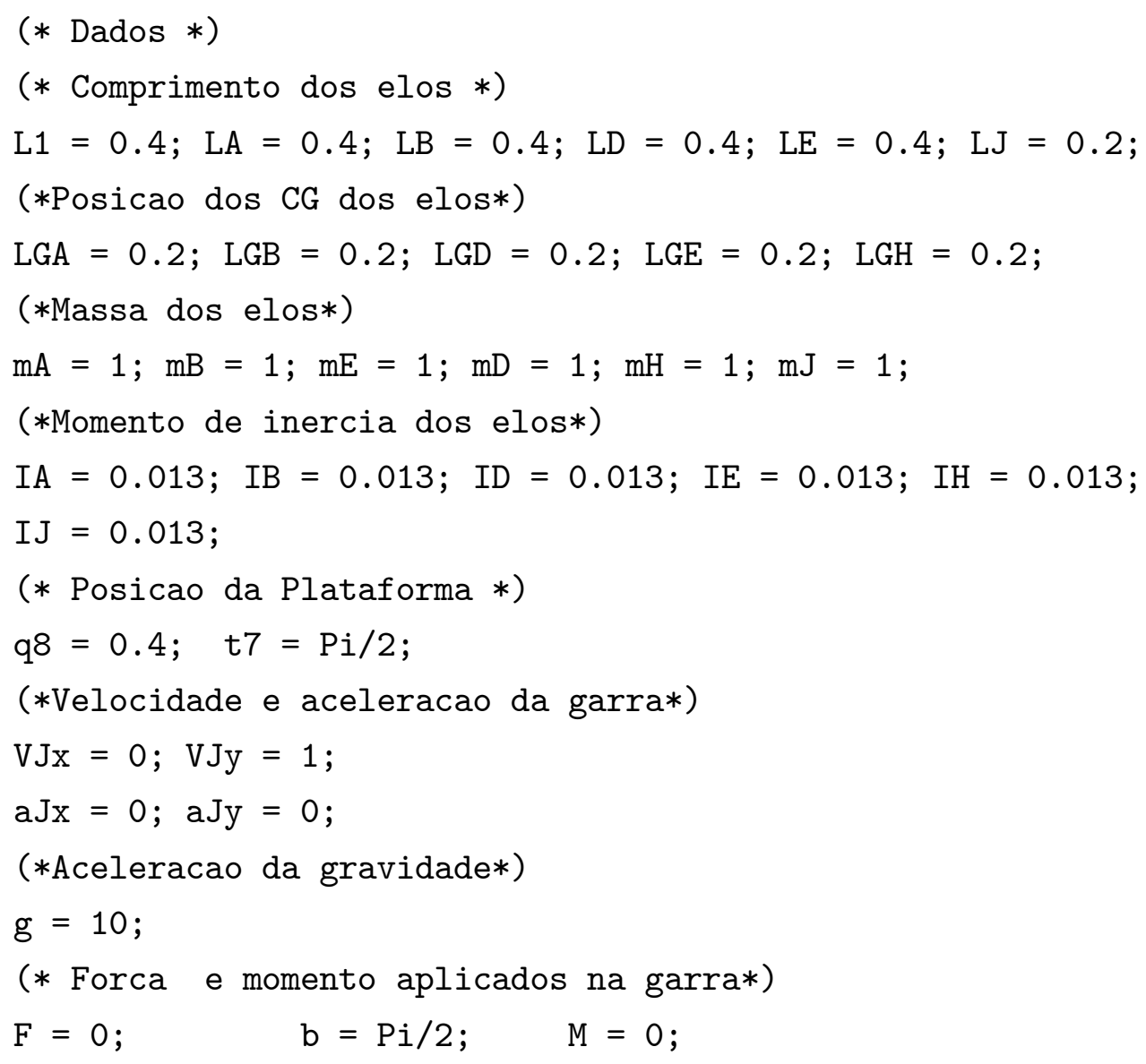









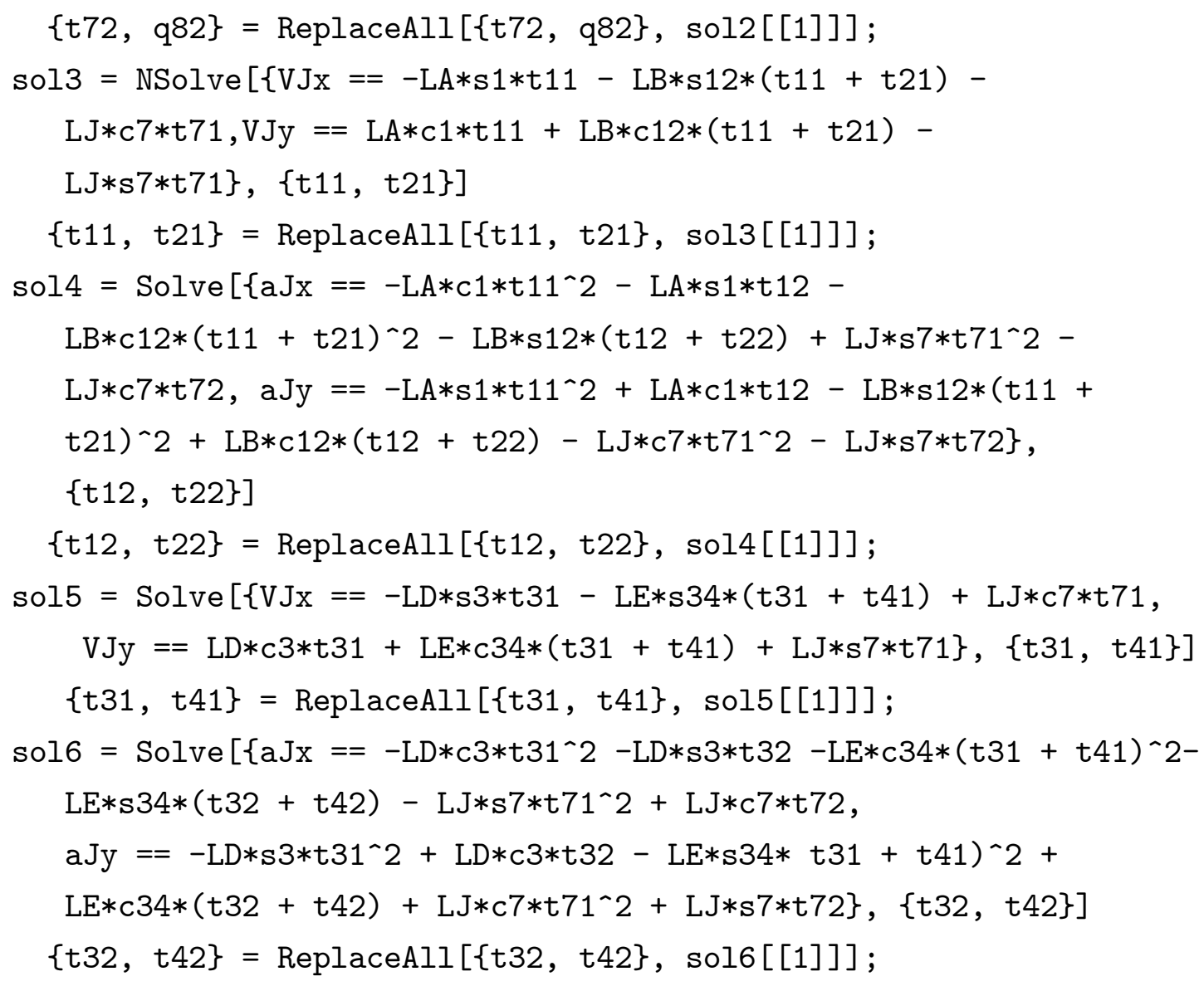

(* CALCULO DAS ACELERACOES DOS CORPOS *)

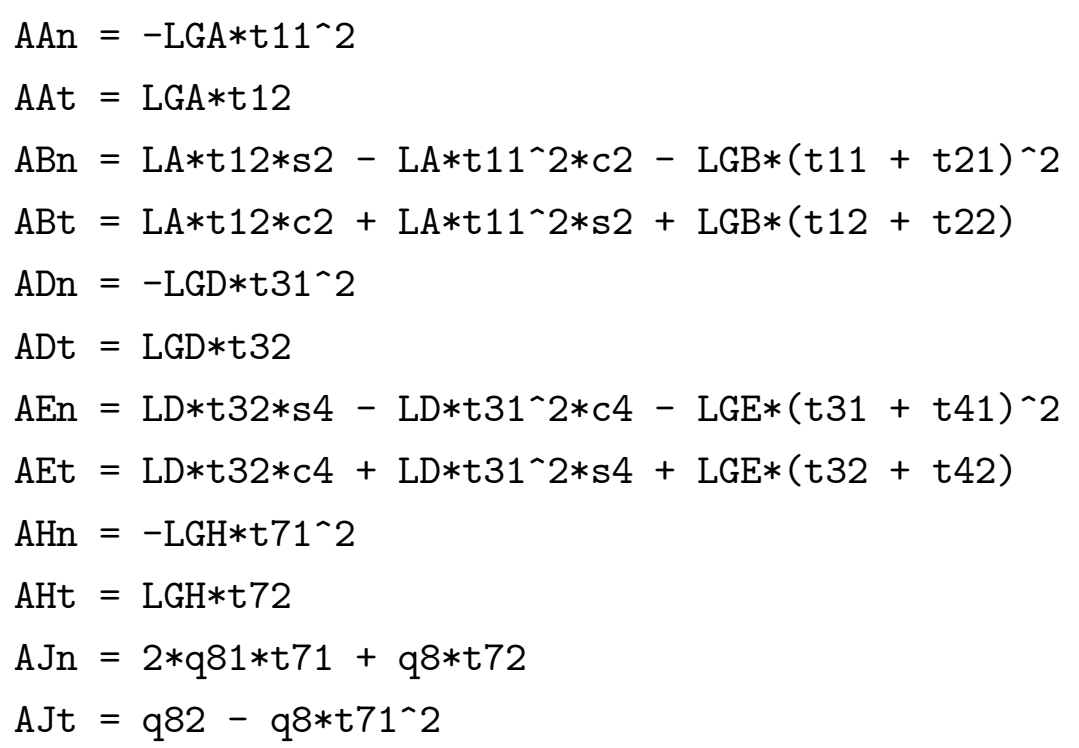

(*Anexo O : Cálculo de T1, T1D*)

$\mathrm{T} 1 \mathrm{D}=\mathrm{ADt} \mathrm{LGD} \mathrm{mD}+\mathrm{c} 3 \mathrm{~g} \mathrm{LGD} \mathrm{mD}+\mathrm{c} 3 \mathrm{~g} \mathrm{LD} \mathrm{mE}+\mathrm{c} 34 \mathrm{~g} \mathrm{LGE} \mathrm{mE}+$ $\mathrm{mE}(\mathrm{AEt} \mathrm{c4} \mathrm{LD}+\mathrm{AEt} \mathrm{LGE}+\mathrm{AEn} \mathrm{LD} \mathrm{s4)} \mathrm{+(ID} \mathrm{+} \mathrm{IE)} \mathrm{t32} \mathrm{+} \mathrm{IE} \mathrm{t42} \mathrm{+}$ 
$(\mathrm{c} 4 \mathrm{LD}+\mathrm{LE})(-\mathrm{AEt} \mathrm{LGE} \mathrm{mE} \mathrm{-} \mathrm{c34} \mathrm{g} \mathrm{LGE} \mathrm{mE} \mathrm{-} \mathrm{IE} \mathrm{t32} \mathrm{-} \mathrm{IE} \mathrm{t42)/LE} \mathrm{+}$ LD s4 (-( (-ABt LGB mB - c12 g LGB mB - IB t12 - IB t22) $((-$ calf $\mathrm{q} 8+$ LJ salf $)(-$ calf +calf kp LJ Sign[q81]/dp)(-calf LJ - q8 salf) (salf -kp LJ salf Sign[q81]/dp)))/ (LB (-2 calf clam LJ - clam q8 salf - calf q8 slam (clam kp LJ q8 salf Sign[q81])/dp)calf kp LJ q8 slam Sign[q81]/ $\mathrm{dp}))$ - ((-AEt LGE mE - c34 g LGE mE - IE t32 - IE t42) $((-$ clam $\mathrm{q} 8+$ LJ slam $)(-$ calf +calf $\mathrm{kp} \mathrm{LJ} \mathrm{Sign[q81]/dp)} \mathrm{-}$ (-calf LJ - q8 salf) (-slam -kp LJ slam Sign[q81]/dp)))/(LE (-2 calf clam LJ - clam q8 salf - calf q8 slam clam kp LJ q8 salf Sign[q81]/dp+calf kp LJ q8 slam Sign[q81]/dp)) $+((\mathrm{M}-\mathrm{AHt} \mathrm{LGH} \mathrm{mH}-\mathrm{c} 7 \mathrm{~g} \mathrm{LGH} \mathrm{mH}-\mathrm{AJn} \mathrm{mJ} \mathrm{q} 8-\mathrm{c} 7 \mathrm{~g} \mathrm{~mJ}$ q8 $\mathrm{cb} F \mathrm{q} 8 \mathrm{~s} 7+\mathrm{c} 7 \mathrm{~F}$ q8 sb - IH t72 - IJ t72) (-calf +) ) + ((M AHt LGH mH - c7 g LGH mH - AJn mJ q8 - c7 g mJ q8 - cb F q8 s7 + c7 F q8 sb - IH t72 - IJ t72) (-calf +calf kp LJ Sign[q81]/dp) (-calf LJ - q8 salf) (c7 cb F - AJt mJ - g mJ s7 + F s7 sb + $(-F p r-k p(M-I J$ t72)/dp) Sign[q81]))/(-2 calf clam LJ clam q8 salf - calf q8 slam -clam kp LJ q8 salf Sign[q81]/dp+ calf $\mathrm{kp} \mathrm{LJ}$ q8 slam Sign[q81]/dp))

$\mathrm{T} 1=$ AAt LGA $\mathrm{mA}+\mathrm{c} 1 \mathrm{~g}$ LGA $\mathrm{mA}+\mathrm{c} 1 \mathrm{~g} \mathrm{LA} \mathrm{mB}+\mathrm{c} 12 \mathrm{~g} \mathrm{LGB} \mathrm{mB}+\mathrm{mB}($ $\mathrm{ABt}$ c2 $\mathrm{LA}+\mathrm{ABt} \mathrm{LGB}+\mathrm{ABn} \mathrm{LA} \mathrm{s} 2)+(\mathrm{IA}+\mathrm{IB}) \mathrm{t} 12+\mathrm{IB} \mathrm{t} 22+$ (c2 LA + LB) (-ABt LGB mB - c12 g LGB mB - IB t12 - IB t22)/LB+ LA s2 (-(-ABt LGB mB - c12 g LGB mB - IB t12 - IB t22) (salf kp LJ salf Sign[q81]/dp)/LB (-calf +calf kp LJ Sign[q81]/dp)(-AEt LGE mE - c34 g LGE mE - IE t32 - IE t42) (-slam kp LJ slam Sign[q81]/dp)/LE (-calf +calf kp LJ Sign[q81]/dp)+ c7 cb F - AJt mJ - g mJ s7 + F s7 sb + (-Fpr -kp (M - IJ t72)/ dp) Sign[q81]/(-calf +calf kp LJ Sign[q81]/dp)-1/(-calf + calf $\mathrm{kp} \mathrm{LJ} \mathrm{Sign[q81]/dp)} \mathrm{((-clam} \mathrm{-clam} \mathrm{kp} \mathrm{LJ} \mathrm{Sign[q81]/dp)}$ $(-((-\mathrm{ABt}$ LGB $\mathrm{mB}$ - c12 g LGB mB - IB t12 - IB t22) ((-calf q8 + LJ salf) (-calf +calf kp LJ Sign[q81]/dp) - (-calf LJ - q8 salf) (salf -kp LJ salf Sign[q81]/dp)))/(LB (-2 calf clam LJ clam q8 salf - calf q8 slam -clam kp LJ q8 salf Sign[q81]/dp+ calf kp LJ q8 slam Sign[q81]/dp)) - ((-AEt LGE mE c34 g LGE mE - IE t32 - IE t42) ((-clam q8 + LJ slam) (-calf +calf kp LJ Sign[q81]/dp) - (-calf LJ - q8 salf) $(-\mathrm{slam}-\mathrm{kp}$ LJ slam Sign[q81]/dp)))/(LE (-2 calf clam LJ - 
clam q8 salf - calf q8 slam -clam kp LJ q8 salf Sign[q81]/dp+ calf kp LJ q8 slam Sign[q81]/dp)) + ((M -AHt LGH mH c7 g LGH mH - AJn mJ q8 - c7 g mJ q8 - cb F q8 s7 + c7 F q8 sb - IH t72 - IJ t72) (-calf + calf kp LJ Sign[q81]/dp) - (-calf LJ - q8 salf) (c7 cb F AJt $m J-g m J s 7+F s 7 s b+(-F p r-k p(M-I J ~ t 72) / d p)$ Sign[q81]))/(-2 calf clam LJ - clam q8 salf - calf q8 slam clam kp LJ q8 salf Sign[q81]/dp+ calf kp LJ q8 slam Sign[q81]/dp)))) 


\section{REFERÊNCIAS BIBLIOGRÁFICAS}

[1] HESS-COELHO, T. A.; BRANCHINI, D. M.; MALVEZZI, F. A new family of 3-DOF parallel robot manipulators for pick-and-place operations In: CONGRESSO INTERNACIONAL DE ENGENHARIA MECÂNICA, 18, 2005. Ouro Preto. Anais...: COBEM 2005, Ouro Preto, 2005 .

[2] REUlEAUX, F. at al. Reuleaux, Kinematics of Machinery, Macmillan, London, Dover Publications, New York, 1963.

[3] SUH, C. H.; RADCLIFFE, C. W. Kinematics and mechanisms design. New York: John Wiley \& Sons, 1978, 434p.

[4] MERLET, J. P. Parallel Robots. Dordrecht: Kluwer Academic Publishers, 2000, 355p.

[5] BONEV, I. ParalleMIC - the Parallel Mechanisms Information Center. Disponível em : <:www.parallemic.org/Terminology/General/html $>$. Acesso em julho de 2005.

[6] SHIGLEY, J. E.; UICKER, J. J. Theory of machines and mechanisms. London: McGraw-Hill, 1980. 577p.

[7] HESS-COELHO, T. A. Topologia, síntese e análise de uma estrutura cinemática paralela. 2005. 63 f. Tese (Livre Docência) - Escola Politénica, Universidade de São Paulo, São Paulo, 2005.

[8] TSAI, L.-W. Robot analysis: the mechanics of serial and parallel manipulators. New York: John Wiley \& Sons, 1999. 505p.

[9] GOSSELIN, C. M.; ANGELES, J. The optimum kinematic design of a planar three-degree-of-freedon parallel manipulator. Journal of Mechanisms, Transmissions and Automation in Design, v. 110, n²1, p 35-41, 1988.

[10] STAMPER, R. E.; TSAI, L.-W.; WALSH, G. C. Optimization of a three DOF translational platform for well-conditioned workspace. In: INT. CONF. ON ROBOTICS AND AUTOMATION, 1977, Albuquerque Proceedings ...Novo Mexico: IEEE, v. 4, p. 3250-3255, 1997. 
[11] MERLET, J. P. Parallel robots: open problems. Disponível em: $<$ http://www.inria.fr/coprin/merlet/merlet_eng.html >. Acesso em: 4 mar. 2006.

[12] BONEV, I.; RYU, J. A new approach to orientation workspace analysis of 6-DOF parallel manipulators. Mecanism and machine theory, v. 36, n. 01, p. 15-28, 2001.

[13] KNAPP, W. ; COBET, M. The IWF hexaglide: a new concept for high speed machining. IWF, Zürich, nov., 2000.

[14] GOSSELIN, C.; ANGELES, J. Singularity analysis of closed-loop chains. IEEE Trans. on Robotics and Automation, June 1990.

[15] MALVEZZI, F. Avaliação do comportamento cinemático de um mecanismo paralelo tridimensional. 2006. 135f. Dissertação (Mestrado) - Escola Politécnica, Universidade de São Paulo, São Paulo, 2006.

[16] TARTARI FILHO, S. C. Modelagem e otimização de um robô de arquitetura paralela para aplicações industriais. 2006, 205 f. Dissertação (Mestrado) - Escola Politécnica, Universidade de São Paulo, São Paulo, 2006.

[17] KOREN, Y. Robotics for engineers, New York: McGraw-Hill Book Company, 1985

[18] GILLESPIE, R.B. Kane's equations for haptic display of multibody systems. v. 3. n.2., Haptics-e, Disponível em <:http://www.haptics-e.org > . Acesso em: 18 ago. 2003.

[19] ITUL, T. P.; PISLA, D. L.; PISLA, A. Dynamic model of a 6-DOF parallel robot by considering friction effects. In: IFTOMM WORL CONGRESS, 12., Besançon. Proceedings ...Besançon (France), p.18-21, 2007.

[20] CRAIG, J. J. Introduction to Robotics. Mechanics and Control. Amsterdam: Addison-Wesley, 1989.

[21] FARHAT, N.; DIAZ, M.-A.; MATA, V. Dynamic parameter identification of parallel robots considering physical feasibility and nonlinear friction models.In: IFTOMM WORL CONGRESS, 12., Besançon. Procedings ... Besançon (France), 2007. 
[22] ASADA, H. ; SLOTINE, J. E. Robot analysis and control. New York: Jonh Willey and Sons, 1986. 